\title{
ALTERNATING CURRENT DIELECTROPHORETIC MANIPULATION OF ERYTHROCYTES IN MEDICAL MICRODEVICE TECHNOLOGY
}

Kaela M. Leonard

Michigan Technological University

Follow this and additional works at: https://digitalcommons.mtu.edu/etds

Part of the Biomedical Engineering and Bioengineering Commons Copyright 2012 Kaela M. Leonard

\section{Recommended Citation}

Leonard, Kaela M., "ALTERNATING CURRENT DIELECTROPHORETIC MANIPULATION OF ERYTHROCYTES IN MEDICAL MICRODEVICE TECHNOLOGY", Dissertation, Michigan Technological University, 2012.

https://doi.org/10.37099/mtu.dc.etds/686

Follow this and additional works at: https://digitalcommons.mtu.edu/etds

Part of the Biomedical Engineering and Bioengineering Commons 


\title{
ALTERNATING CURRENT DIELECTROPHORETIC MANIPULATION OF ERYTHROCYTES IN MEDICAL MICRODEVICE TECHNOLOGY
}

\author{
By \\ Kaela M. Leonard

\begin{abstract}
A DISSERTATION
Submitted in partial fulfillment of the requirements for the degree of DOCTOR OF PHILOSOPHY

(Chemical Engineering)

MICHIGAN TECHNOLOGICAL UNIVERSITY

2012

(C) 2012 Kaela Leonard
\end{abstract}



This dissertation "Alternating Current Dielectrophoretic Manipulation of Erythrocytes in Medical Microdevice Technology," is hereby approved in partial fulfillment of the requirements for the Degree of DOCTOR OF PHILOSOPHY IN CHEMICAL ENGINEERING.

Department of Chemical Engineering

Signatures:

Dissertation Advisor

Adrienne R. Minerick

Department Chair

S. Komar Kawatra

Date 

For Emily

Were it not for you, I wouldn't be doing this

Ad astra per aspera 



\section{Table of Contents}

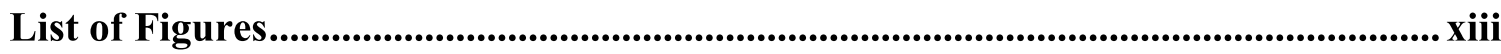

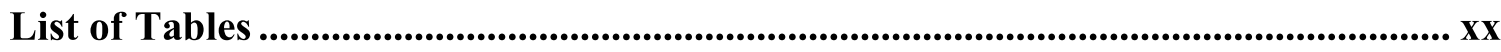

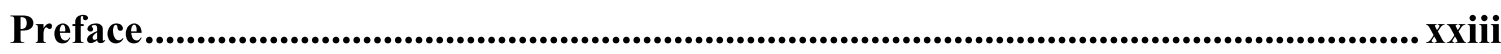

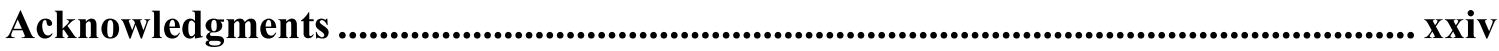

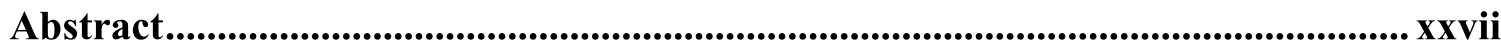

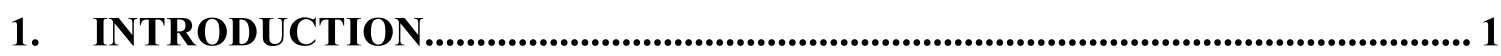

1.1. Background: Microdevices, Electrokinetics and Human Erythrocytes ....................2

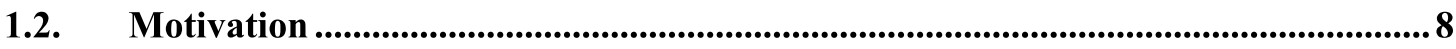

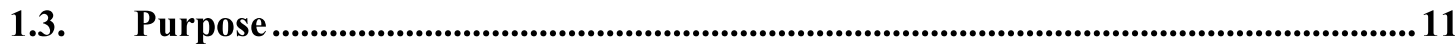

2. Human Blood System: Biochemistry and Electrokinetic Manipulation............. 15

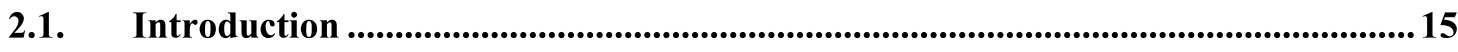

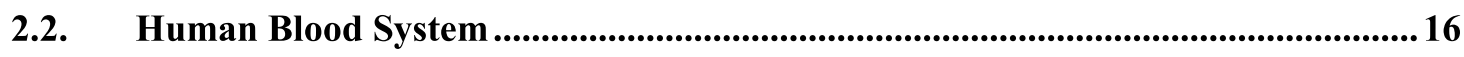

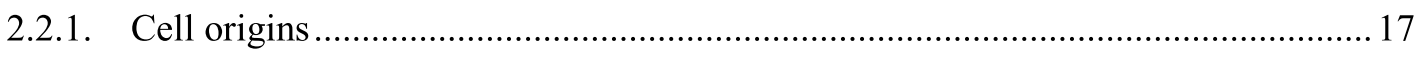

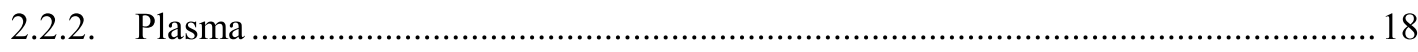

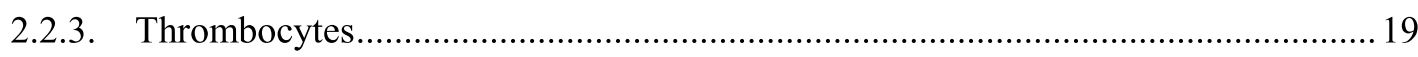

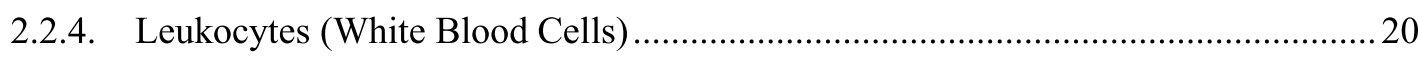

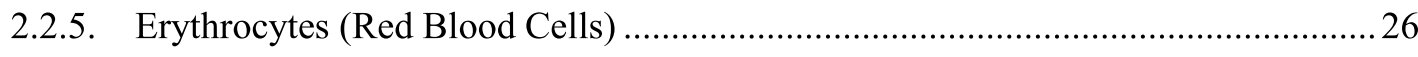

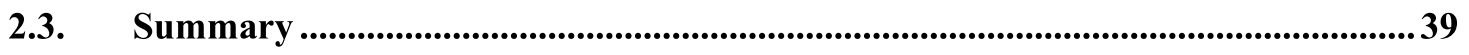




\section{ELECTROKINETICS AND DIELECTROPHORESIS: THEORY AND USE}

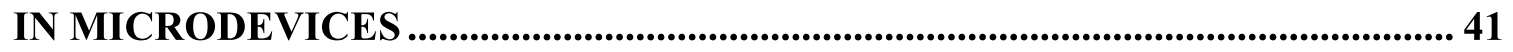

3.1. Introduction ......................................................................................................... 41

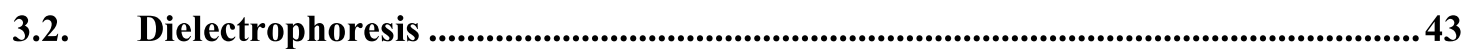

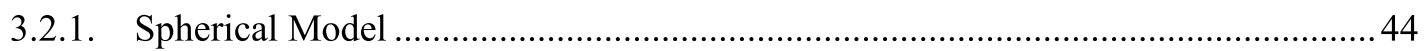

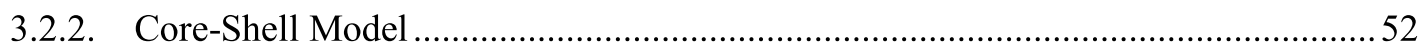

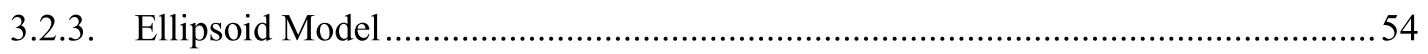

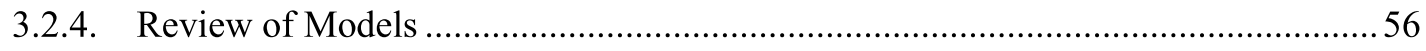

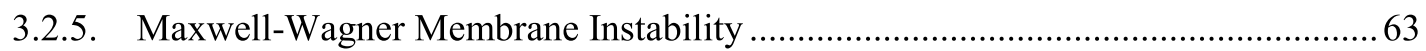

3.3. Dielectrophoresis in Microdevices …………………..................................................65

3.4. Summary ……..........................................................................................................68

4. Alternating Current Dielectrophoretic Characterization of Human Erythrocyte Lysis in a Medical Microdevice ............................................................................. 71

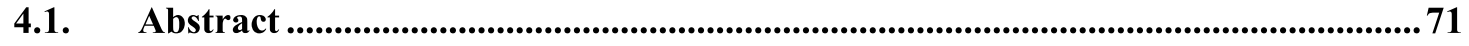

4.2. Introduction .............................................................................................................. 72

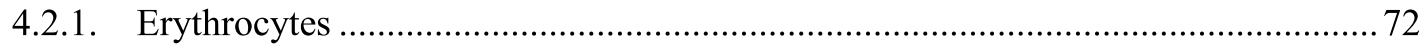

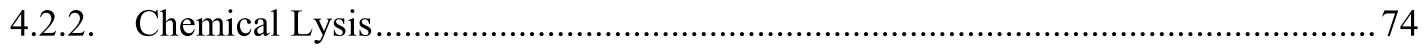

4.2.3. Mechanical Lysis ...........................................................................................

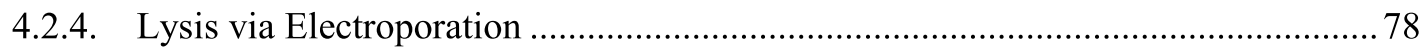

4.2.5. Lysis via Alternating Current Dielectrophoresis......................................................79

4.3. Materials and Methods .................................................................................................8

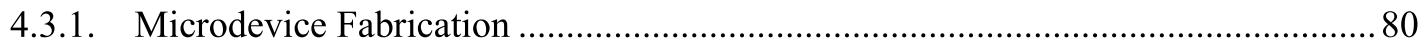

4.3.2. Erythrocyte Sample Preparation............................................................................. 81

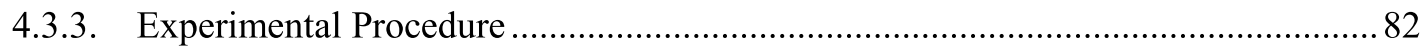




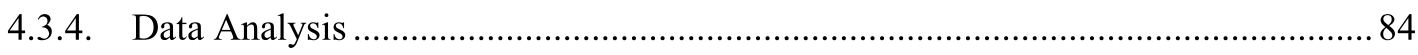

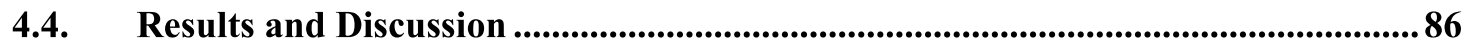

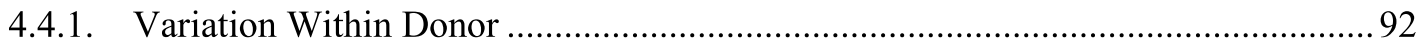

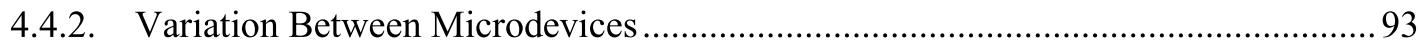

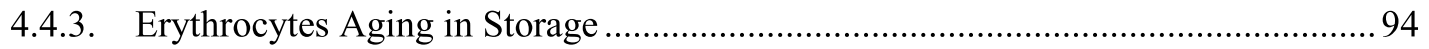

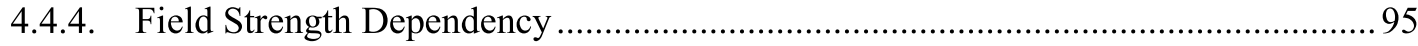

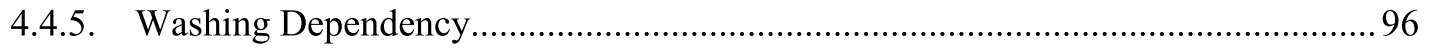

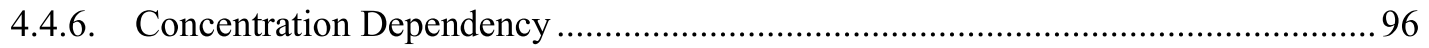

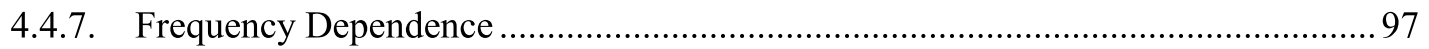

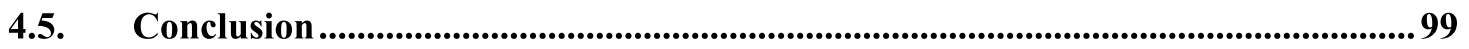

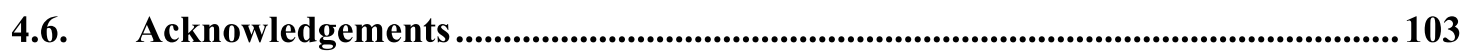

5. Explorations of ABO-Rh Antigen Expressions on Erythrocyte

Dielectrophoresis: Changes in Cross-over Frequency ${ }^{1}$............................................ 105

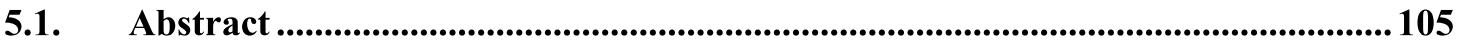

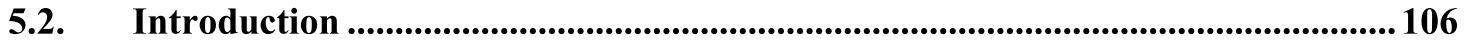

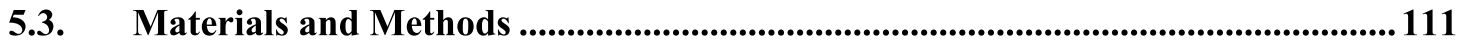

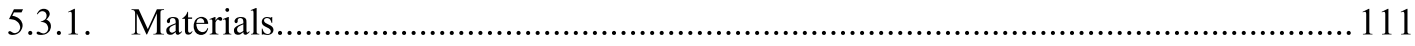

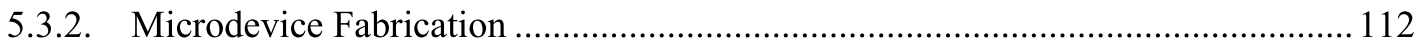

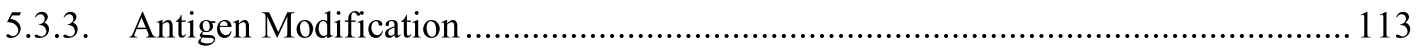

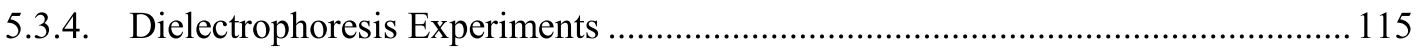

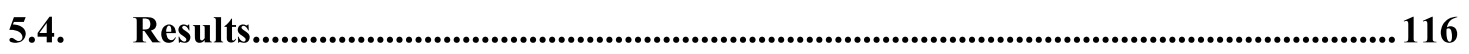

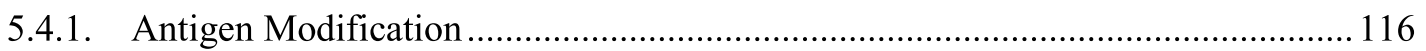

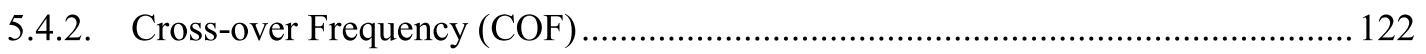

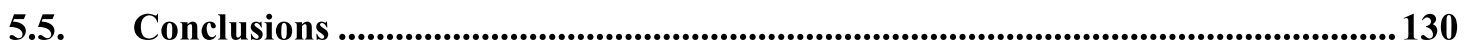


5.6. Acknowledgements

6. Dielectrophoretic Response of Human Erythrocytes Using AC Sweeps: ABO-

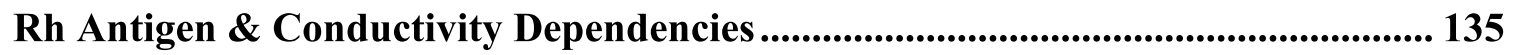

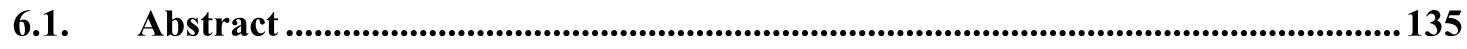

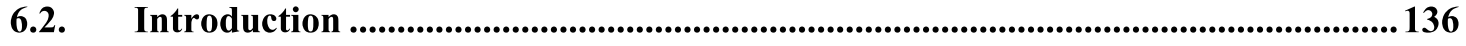

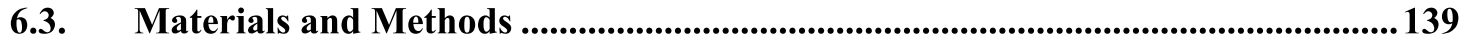

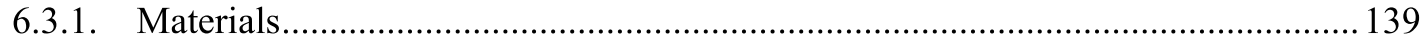

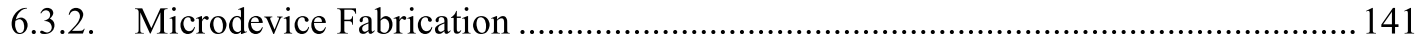

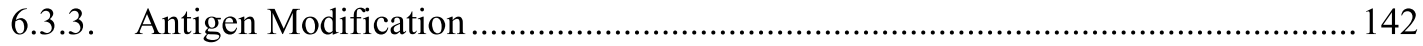

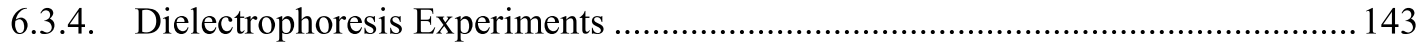

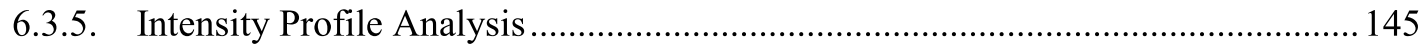

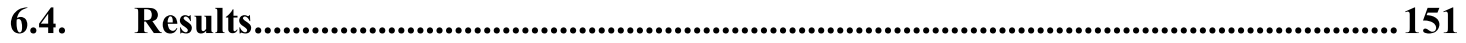

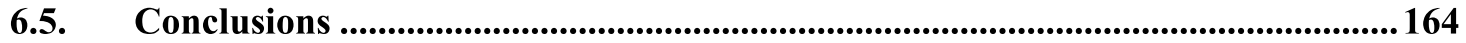

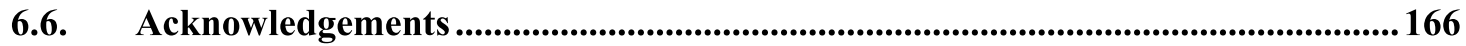

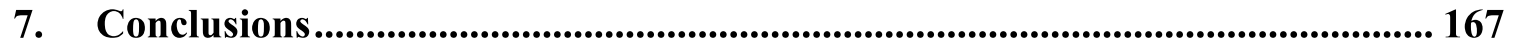

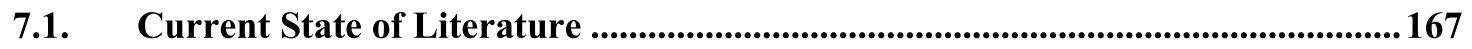

7.2. Low kHz Erythrocyte Rupturing ...............................................................................169

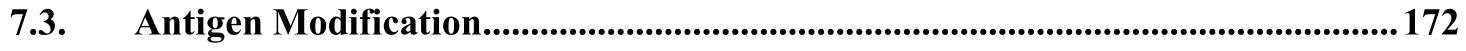

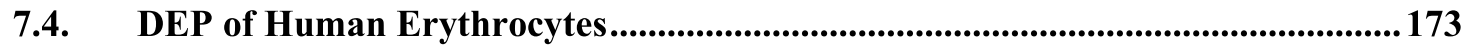

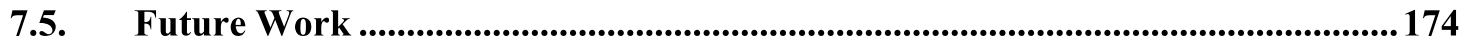

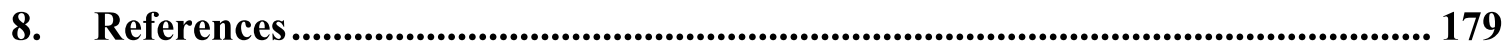

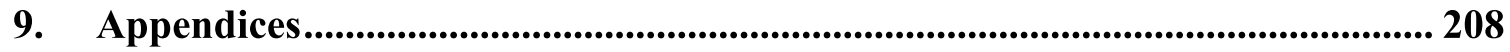

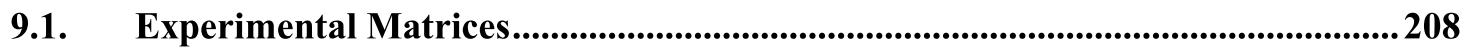


9.1.1. Chapter 6 208

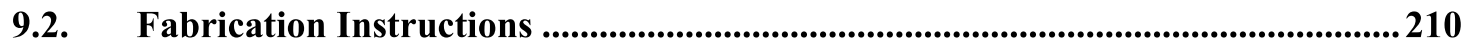

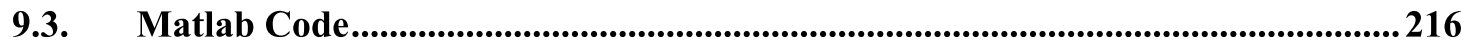

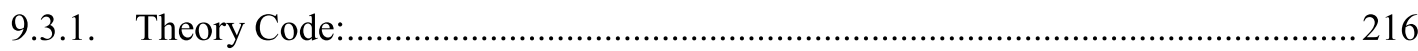

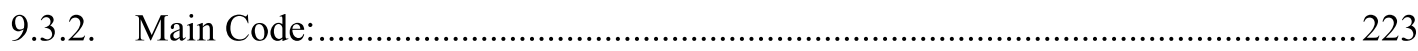

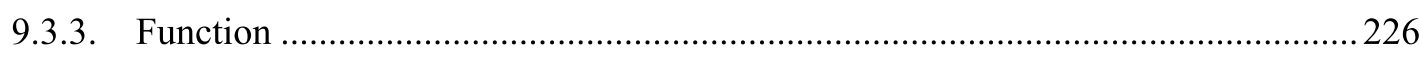

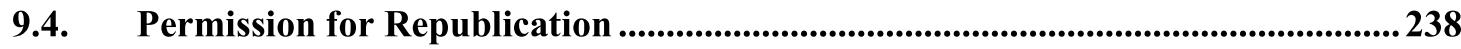

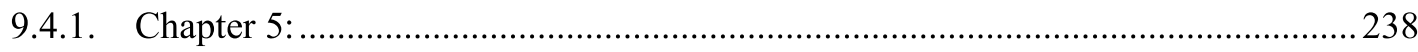

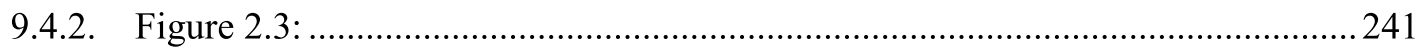

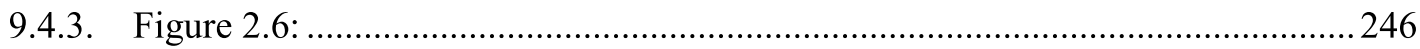

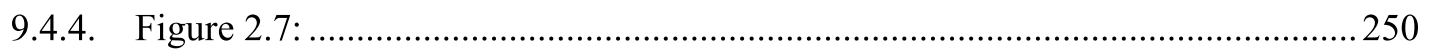

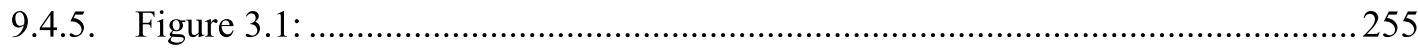





\section{List of Figures}

Figure 2.1. Blood and Tissue Cells derived from a common Hematopoietic stem cell.

Information from ${ }^{132}$

Figure 2.2. Graphical representation of the differences in dielectrophoretic crossover frequency (in $\mathrm{MHz}$ ) based on medium conductivity and the following subpolymorphonuclear leukocytes: B lymphocytes (-), Eosinophils (u), Basophils (n), Neutrophils $(\square$, T lymphocytes $(\times)$, and Monocytes $(*)$. Data taken from: Vykoukal, D. M., Gascoyne, P. R. C., Vykoukal, J., Integrative Biology 2009, 1, $477-484$.

Figure 2.3. SEM Images of T-lymphocytes (A), B-lymphocytes (B), Monocytes (C) and Granulocytes (D). The scale bar corresponds to $20 \mu \mathrm{m}$. Reprinted with permission from $^{14}$ 26

Figure 2.4. Major proteins and antigens of the human erythrocyte membrane. On the left is $\mathrm{O}-$, showing the $\mathrm{O}$ antigen and lack of Rhesus factor. On the right is $\mathrm{AB}+$ showing both the $\mathrm{A}$ and $\mathrm{B}$ antigens and the transmembrane Rhesus factor. Also drawn is the spectrin dimer, which provides support and stability for the membrane.

Figure 2.5. Carbohydrate structures of $\mathrm{O}, \mathrm{A}$ and $\mathrm{B}$ antigens as they appear on a human erythrocyte. The glycoprotein attaches the carbohydrate structure to the cell membrane $e^{120,121}$. 
Figure 2.6. Biophysical and dielectric property differences between healthy and parasitized human erythrocytes. Summarized from ${ }^{4}$. Reprinted with kind permission from ${ }^{5}$

Figure 2.7. Crossover Frequency of healthy human erythrocytes (open symbols) and human erythrocytes infected with Plasmodium falciparum (closed symbols). Reprinted with kind permission from ${ }^{4}$ 38

Figure 3.1. Guide to frequency regions at which specific DEP behaviors typically occur, as well as information about what methodology is appropriate in these regions. Reprinted with permission from ${ }^{164}$

Figure 3.2. Basic diagram showing the electric field on each dipole side. The force on the dipole is the electric fields are multiplied by the charge on the dipole half and summed.

Figure 3.3. Representation of a dielectric sphere in a dielectric medium that is used to solve for the electrostatic potential.

Figure 3.4. Theoretical plots corresponding to the four models discussed above Spherical (A), Shelled Spherical (B), Ellispoidal-Minor (C) and Elllipsoidal-Major (D). Shown are the three medium conductivities tested: $0.01 \mathrm{~S} / \mathrm{m}$ (red), $0.1 \mathrm{~S} / \mathrm{m}$ (green) and $0.9 \mathrm{~S} / \mathrm{m}$ (blue). Both the Shelled Spherical and the Ellipsoidal models show the two-COF behavior that is typical of cells due to their double dielectric nature. 59

Figure 3.5. Theoretical graphs of major (solid) and minor (dashed) axis ellipsoidal models. Shown for three representative medium conductivities: $0.01 \mathrm{~S} / \mathrm{m}(\mathrm{A})$, $0.1 \mathrm{~S} / \mathrm{m}(\mathrm{B})$ and $0.9 \mathrm{~S} / \mathrm{m}(\mathrm{C})$. These conductivities correspond to those used in 
experimental section. The minor axis hits both COFs at a higher frequency than the major axis. 62

Figure 4.1. Side (A) and Top (B) views of the microdevice on the microscope stage with alligator clips leading to AC Generator. AutoCAD mask of complete fluidic layer (C) and magnified view of the lysis chamber (D). Labels are 1: Inlet, 2: Outlet, 3 : Horizontal grounded electrode, 4: Vertical active electrode and 5: lysis chamber... 81 Figure 4.2. Time dependent rupturing for $\mathrm{B}+$ manual versus computer (A), all computeranalyzed $\mathrm{B}+$ donors $(\mathrm{B})$ and the average profile for all blood types tested (C). Shown in (D) is the fraction of cells ruptured at 5, 10 and 15 minutes along with $95 \%$ confidence intervals. Statistically relevant differences at a $95 \% \mathrm{CI}$ are indicated by * 87

Figure 4.3. Graph showing kinetic fit for A+ (n), A- $(\square)$ and B+ $(\bullet)$ based on Equation (3). The erythrocytes are assumed to follow a first order reaction rate where the species of interest is the number of unruptured blood cells versus time. 92

Figure 4.4. Final cell rupture fraction, $\mathrm{X}_{\mathrm{f}}$, for the dependencies: Erythrocyte storage age (A), Electric field strength (B), Washing,(C) and Cell concentration (D). For clarity, 95\% confidence bars were calculated but are not shown for storage age (A) and field

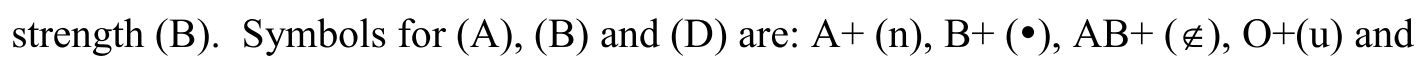
O- ( ). Bars in (C) and (D) represent 95\% confidence intervals. 97

Figure 4.5. Final cell rupture fraction, $\mathrm{X}_{\mathrm{f}}$ versus applied frequency for $\mathrm{A}+$ (red square), $\mathrm{A}$ - (blue square), $\mathrm{AB}+$ (red triangle), $\mathrm{AB}-$ (blue triangle), $\mathrm{O}+$ (red diamond) and $\mathrm{O}-$ (blue diamond) 98 
Figure 5.1. Enzymatic reaction recipe and procedure. Dilution conditions are given for both native and modified blood, which are separately loaded into the microdevice. The gold-plated quadrapole electrodes (width of $100 \mathrm{~mm}$ and spacing of $50 \mathrm{~mm}$ ) in the PDMS-glass microdevice are connected to an AC generator and erythrocyte frequency responses are recorded with 10x and20x LabSmith video microscopy. Responses are categorized based on the positive and negative dielec- trophoresis patterns shown.

Figure 5.2. (A) UV-Vis analysis of effluent from red blood cells modified with b(1-3)galactosidase under four different reaction conditions. (B) UV-Vis traces of the enzyme reaction components (BSA, b-galactosidase, $\mathrm{NaCl}, \mathrm{G} 2$ ) demonstrating that these do not contribute to peaks from 300 to $900 \mathrm{~nm}$. (C) Representative HPLC trace for galactose standard.

Figure 5.3. Microscope images of native and modified B- blood agglutination results at 25x. Native B- blood (A) without serum, (B) with anti-A serum and (C) with anti-B serum. $\beta(1,3)$-Galactosidase-modified B- blood (D) without serum, (E) with anti-A serum and (F) with anti-B serum. (C) shows B- agglutination with a substantially attenuated cell agglutination is observed in $(\mathrm{F})$. The enzyme damages membrane integrity so intact cell counts drops upon digestion

Figure 5.4. 20x Microscopy images of native and modified B- experiencing DEP. The first row shows native erythrocytes at $100 \mathrm{kHz}(\mathrm{A}), 42 \mathrm{MHz}$ (B) and $80 \mathrm{MHz}$ (C), whereas the second row shows $\beta$-galactosidase-modified erythrocytes at the same frequencies (D)-(F). At $100 \mathrm{kHz}$, native (A) and modified (D) both show nDEP. At $42 \mathrm{MHz}$, native erythrocytes (B) experience nDEP and pDEP (out of focus) while 
the modified erythrocytes (E) experience very weak nDEP and pDEP as would be expected near the COF. $80 \mathrm{MHz}$ images show nDEP for both (C) and (F). The white arrows point to erythrocytes located in the center or away from the electrodes for nDEP (B) and near the electrode surface for pDEP (E)....................................... 123

Figure 5.5. (A) Negative (black bars) and positive (gray bars) dielectrophoresis ranges for native (left side) and modified (right side) erythrocytes. COF was calculated as the midpoint of the concurrent $\mathrm{nDEP} / \mathrm{pDEP}$ range. (B) Lower COF range (30-55 MHz) showing $\mathrm{nDEP}(\boldsymbol{\square})$, pDEP $(\circ)$ or both $(\mathbf{\square}+0)$ behaviors. Differences are discernable with $\mathrm{ABO}$ antigen expression. 126

Figure 5.6. A more traditional plot of $\mathrm{nDEP}, \mathrm{COF}$ and $\mathrm{pDEP}$ behaviors as a function of frequency. Plot (A) demonstrates a $\mathrm{COF}$ difference between native $\mathrm{AB}+, \mathrm{O}+$ and $\mathrm{O}-$ that is negated after modification (B). Graph (C) illustrates donor reproducibility between native and modified $\mathrm{AB}+$. (D) The narrowing of the lower COF range from the native (closed symbols) to the modified (open symbols) erythrocytes. 129

Figure 6.1. Fabrication of microdevices: UV exposure of SU-8 covered silica wafer with UV mask (A), developing of photoresist (B), final wafer showing quadrapole chamber design (C), titanium-gold plated electrodes made via sputter system (D), PDMS casting of chamber (E) and final device showing copper wires used to connect to alligator clips and silver epoxy that connects copper wires to plated electrodes (F). Zeiss view window (for 25X magnification) of electrode area (G). Example of behavior for $\mathrm{nDEP}(\mathrm{H})$ and $\mathrm{pDEP}(\mathrm{I})$ response 142

Figure 6.2. Native (A) and O- (B) erythrocytes suspended in $0.1 \mathrm{~S} / \mathrm{m}$ dextrose buffer and subjected to a $2.5 \mathrm{~V}_{\mathrm{pp}}$ signal swept from $100 \mathrm{kHz}$ to $1.9 \mathrm{MHz}$ over 400 seconds. At 
$0.27 \mathrm{MHz}$ both samples are experiencing nDEP. The transition to pDEP occurs at $0.79 \mathrm{MHz}$ for A- and $1.35 \mathrm{MHz}$ for O-. A period of strong pDEP occurs at $1.69 \mathrm{MHz}$ for A- and 1.62MHz for O-. Transition back to nDEP occurs at $1.86 \mathrm{MHz}$ for A- and 1.71 MHz for O-. This demonstrates visually observable differences in blood cell motion by $\mathrm{ABO}$ type.

Figure 6.3. (A) COMSOL simulation of of gradient of Electric field squared for quadrapole device showing Downward, Upward, Horizontal and Vertical profile line positions. Diagonal (B) and Horizontal/Vertical Bias (C) used in the calculation of the biased intensity from the raw intensity values. 146

Figure 6.4. Video frames of $\mathrm{B}+$ cell behaviors in the dielectrophoretic microdevice at the noted frequencies (A) and the corresponding intensity profile oriented from top left electrode to bottom right electrode at the same frequency (B)

Figure 6.5 . Video images of of native $\mathrm{O}$ - sample in $0.1 \mathrm{~S} / \mathrm{m}$ medium during the $100 \mathrm{kHz}$ to 1.9MHz in 400 s sweep: $352 \mathrm{kHz}(\mathrm{A}), 424 \mathrm{kHz}(\mathrm{B}), 1.7 \mathrm{MHz}(\mathrm{C}), 1.78 \mathrm{MHz}(\mathrm{D})$ and $1.83 \mathrm{MHz}(\mathrm{E})$. Intensity absolute values (blue lines) for three different standard error tolerance levels: 0.5 (F-J), $0.25(\mathrm{~K}-\mathrm{O})$ and $0.1(\mathrm{P}-\mathrm{T})$, each at the corresponding frequencies. The red lines are the upward diagonal bias curve. The biased intensity value from Equation (3) is noted on each plot.

Figure 6.6. Biased intensity for all four profile lines: Downward (blue), Upward (red), Horizontal (green) and Vertical (purple), as well as the Combined Intensity (black) for the three standard error tolerance levels: 0.5 (A), 0.25 (B) and 0.1 (C). Dashed lines correspond to frequencies featured in Figure 6.5: 1. 352kHz, 2. $424 \mathrm{kHz}, 3$. 1.7MHz, 4. $1.78 \mathrm{MHz}$ and $5.1 .83 \mathrm{MHz}$. 
Figure 6.7. DEP response predictions for a $\mathrm{B}+$ donor. Green lines correspond to the native sample and purple the digested. Closed symbols are $0.01 \mathrm{~S} / \mathrm{m}$ medium conductivity while open symbols are $0.1 \mathrm{~S} / \mathrm{m}$ medium conductivity. Conductivity comparisons are in A (native) and B (modified) while native and modified are compared for the $0.01 \mathrm{~S} / \mathrm{m}(\mathrm{C})$ and $0.1 \mathrm{~S} / \mathrm{m}$ (D) medium conditions.

Figure 6.8. Experimentally predicted DEP response for a given blood type at two medium conditions and two cell treatement conditions: native at $0.01 \mathrm{~S} / \mathrm{m}(\mathrm{A})$, modified at $0.04 \mathrm{~S} / \mathrm{m}(\mathrm{B})$, native at $0.1 \mathrm{~S} / \mathrm{m}(\mathrm{C})$, and modified at $0.1 \mathrm{~S} / \mathrm{m}(\mathrm{D})$. The positive blood types are in red and the negative blood types are in blue. Native cells are filled symbols and the modified cells have open symbols.

Figure 6.9. Video images (A) of native $\mathrm{O}-$ blood in $0.1 \mathrm{~S} / \mathrm{m}$ medium at $500 \mathrm{kHz}$ for $0.5 \mathrm{x}$, $1 \mathrm{x}$ and $2 \mathrm{x}$ sweep. DEP Response curves (B) for $0.25 \mathrm{x}, 0.5 \mathrm{x}, 1 \mathrm{x}$, and $2 \mathrm{x}$ sweep rates. The $0.25 \mathrm{x}$ sweep is noisy with limited frequency range and the $2 \mathrm{x}$ sweep is so rapid that the cells experience pDEP when no other sweep registers this behavior. DEP response curves for two different $1 \mathrm{x}$ sweeps $(100 \mathrm{kHz}$ to $1.9 \mathrm{MHz}$ in $400 \mathrm{~s})$ which demonstrates inconsistent behavior between runs. Slowe sweep rates yield more consistent behaviors. 


\section{List of Tables}

Table 2.1. Types and functions of leukocytes. Information from ${ }^{132}$.............

Table 2.2. International Society of Blood Transfusions 30 recognized blood typing systems and their corresponding epitopes. Information from ${ }^{127}$ 34

Table 3.1. Table showing the dielectrophoretic force equation equation and the Clausius-Mossotti factor for each of the models discussed in this chapter.

Table 3.2. Shows COF values (in MHz) for the nDEP to pDEP transition and the pDEP to nDEP transition for the four models graphed above in Figure 3.4. Values were calculated by fitting a linear equation between the two points closest to zero as calculated by Matlab.

Table 4.1. Experimental Plan for antigen, field, washing, concentration, and frequency dependencies tested. Columns refer to individual experiments and rows are experimental parameters within that experimental set. Bold parameter is the parameter of interest for that set of experiments.

Table 4.2. Final rupturing fraction for seven blood types tested and the four donors with the repeat donor and the repeat donor with a new device. Missing cells correspond to tests that either couldn't be run or were deemed statistical outliers. 91

Table 4.3. Kinetic reaction models and rate constants for $\mathrm{A}+, \mathrm{A}-$ and $\mathrm{B}+$, which experienced enough rupture to fit to a reaction model.

Table 4.4. Final rupturing five blood types that had donor and device dependency tested as well as the variation between the two donations from the same donor and the two devices from the same day of donation 94 
Table 4.5. Single factor ANOVA for erythrocyte storage age dependency. $\mathrm{F}$ and $\mathrm{F}_{\text {critical }}$ values were used to determine validity of the null hypothesis at an alpha of 0.05 . Green shading indicates tests where age did not alter final rupture values while red

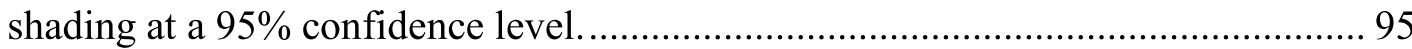

Table 5.1. Conductivity of whole blood $(\mathrm{S} / \mathrm{m})$ based on calculations from Vissers ${ }^{133}$. Pure plasma shown to have conductivity of $1.57 \mathrm{~S} / \mathrm{m}$. 112

Table 5.2. HPLC elution times and peak heights for four standards (fucose, galactose, (13) galactosidase and A-trisaccharide) and four trail erythrocyte modification effluents $(\mathrm{A}+0.2 \mathrm{U} / \mu \mathrm{L} \beta(1-3)$ galactosidase $1 \mathrm{hr}, \mathrm{A}+1 / 2 \mathrm{X} \beta(1-3)$ galactosidase $1 \mathrm{hr}$, $\mathrm{A}+0.1 \mathrm{U} / \mu \mathrm{L} \beta(1-3)$ galactosidase $1 / 2 \mathrm{hr}$, and $\mathrm{B}-0.1 \mathrm{U} / \mu \mathrm{L} \beta(1-3)$ galactosidase $1 / 2 \mathrm{hr}$.)

Table 5.3. Low and high COF for the native and modified blood types tested. Average, standard deviation and range are computed. Average low and high COF are comparable between native and modified erythrocytes. However, the low COF range for the native erythrocytes is $17 \mathrm{MHz}$, whereas after modification this range drops to $5 \mathrm{MHz}$. The range for the high $\mathrm{COF}$ only drops by $2 \mathrm{MHz}$ indicating that this value is not controlled by the expression of $\mathrm{ABO}$ antigens. 130

Table 6.1. Table describing the reactants used in creating the four different medium solutions used. Also included is the final conductivity of each solution. 140

Table 6.2. 24 sweep (six per solution) that were run for these experiments. Notice that the low conductivity solutions were run with the same six sweeps and the higher conductivity solutions were run with another set of six sweeps. For each solution 
two of the sweeps were repeated, explaining why two sweeps for each set show up

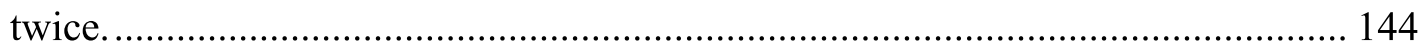

Table 6.3. Sweeps used to test for rate dependency. Each sample was run for at least one

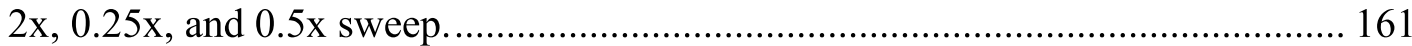




\section{Preface}

The work presented in Chapter 5 has been previously published in

Electrophoresis. The author of this dissertation performed all experiments for the work presented in that article and completed the data analysis. Writing was a joint effort between the author and her advisor Dr. Adrienne Minerick. 


\section{Acknowledgments}

I've been so lucky to have such wonderful support over the past five years that I don't know how I'll thank everyone. First and foremost, I need to thank my family. Tim, AnnMarie, Ryan, Alex and all of my many extended family members have made me feel like I had a whole army in my corner. You can't buy that kind of support. A special thanks to my Nana, who for years has listened to me talk away as I cook dinner or do laundry or walk up the hill to my house. You and Grampie put countless books in my hands as a small child and look where it got me.

I've also been fortunate to have two different dissertation committees at both Mississippi State University and Michigan Technological University. A special thanks goes to those people that read and listened to me present my qualifier, $\mathrm{PhD}$ proposal and now my dissertation: Dr. Mark Bricka, Dr. Shane Burgess, Dr. Priscilla Hill, Dr. James Warnock, Dr. Jason Keith, Dr. Dan Crowl, Dr. Ching-An Peng and Dr. Jeff Allen. In addition to the helpful advice for these committee members, I'd also like to acknowledge the information gleaned from conversations with Ms. Karyn Fay, Dr. Michael Gretz and Dr. Wan Jin Jang regarding the modification of human erythrocytes to remove ABO antigens. There has been no one more helpful in advancing the state of my microdevices by introducing me to newer fabrication methods and mentoring me through the fabrication process than Mr. Bill Knudsen. Additional thanks goes to Dr. Edwar Romero and Dr. Daw Don Cheam for their hours spent mentoring me through the microfabrication process so I could get started on my own microdevices quickly after moving to Michigan Technological University.

A special thanks goes to Dr. Adrienne Minerick for her incredible support and mentorship. She has provided me with ample opportunity to present my research to the greater scientific community, to mentor students younger than me and to enhance my own research. In part because of her mentoring in these areas, I feel confident in my skills as a researcher and prepared for the next step in my career.

Without the help of a huge anonymous list of blood donors, I wouldn't have been able to complete any of the work presented herein. Thank you to each and every single person who has ever donated blood for my project. 
One of the greatest parts of my graduate school experience has been interacting with my peers. On a personal level I owe a great deal of thanks to Amy Parker and Jaclyn Hall for all of their support and the many hours spent over food or coffee discussing life in graduate school. They have been a constant source of support for me, even after we moved from Mississippi State. Thanks also to all of my friends who have not gone through this graduate school journey with me but have listened to frantic phone calls, emails and text messages when things were not working or when things finally decided to work.

In addition to the graduate students I've worked with I've also had the wonderful opportunity to act as a mentor for 16 undergraduate students. Thank you to the following for all of your hard work in the lab, all of the help you provided with data analysis and all you've taught me about how to be a better mentor: Hunter Merrill, Blake Garrett, Alyssa McGuire, Kelsey Maijala, Anell Pullen, Ryan Hill, Courtney Lentowich, Philip Jamison, John Yurgil, Ellesse Bess, Carlos Prado, Carl Baker, Kailey Feuerstein, Kelly-Anne Zayan, Sean Duke and Eric Rutan.

I've been fortunate enough to have wonderful and knowledgeable coworkers during my time in graduate school as well. What I am thanking them for now is only a small portion of what they've actually done for me. Thanks to Tayloria Adams for helpful discussions regarding wording and presentation of processes, since our projects have such similarities between them. Thank you to Ran An for finally explaining circuitry to me so that I could understand why certain resistors were not working. Thank you to Hector Moncada for the many discussions regarding why different cell types behave differently and for the ideas on how to push microdevice fabrication even further. Thanks to Dr. Soumya Srivastava, the years spent sharing an office lead to discussions that advanced both of our projects and no one since has quite understood my problems with blood donors. To CJ Yang, I owe a great deal of thanks for all the hours you spent just listening to me talk out the problems I was having with my experiments. Sometimes just talking out a problem to someone and having them ask pointed questions, solves the problem quite easily. A special thanks goes to Aytug Gencoglu. We started together and we're finishing together and I can't imagine it any other way. The discussions we've had 
over the years have pushed my work further than I could imagine and sometimes just turning my chair to talk to you helped me solve an experimental problem immediately.

My final thanks must go once again to my immediate family (Mom, Dad and Ryan). I know this has been an incredibly long and difficult year for them as well as me and I could not have completed this journey without their love and support. I was incredibly lucky to be born to two parents who put academics above anything else and who told me from a very young age that I could do anything I wanted and no dream was too big. Thank you doesn't seem to say enough.

Thank you again to all of those listed above and any who I may have forgotten. Your support, encouragement and enthusiasm has not gone unnoticed or unappreciated. 


\section{Abstract}

Medical microdevices have gained popularity in the past few decades because they allow the medical laboratory to be taken out into the field and for disease diagnostics to happen with a smaller sample volume, at a lower cost and much faster. Blood is the human body's most readily available and informative diagnostic fluid because of the wealth of information it provides about the body's general health including enzymatic, proteomic and immunological states. The purpose of this project is to optimize operating conditions and study $\mathrm{ABO}-\mathrm{Rh}$ erythrocytes dielectrophoretic responses to alternating current electric signals. The end goal of this project is the creation of a relatively inexpensive microfluidic device, which can be used for the ABO-Rh typing of a blood sample. This dissertation presents results showing how blood samples of a known ABORh blood type exhibit differing behavior to the same electrical stimulus based on their blood type. The first panel of donors and experiments, presented in Chapter 4 occurred when a sample of known blood type was injected into a microdevice with a T-shaped electrode configuration and the erythorcytes were found to rupture at a rate specific to their ABO-Rh blood type. The second set of experiments, presented in Chapter 5, were originally published in Electrophoresis in $2011^{1}$. Novel in this work was the discovery that treatment of human erythrocytes with $\beta$-galactosidase successfully removed $\mathrm{ABO}$ surface antigens such that native A and B blood no longer agglutinated with the proper antibodies. This work was performed in a medium of conductivity $0.9 \mathrm{~S} / \mathrm{m}$ which is close to the measured conductivity of pooled plasma $(\sim 1.1 \mathrm{~S} / \mathrm{m})$. The ability to perform dielectrophoresis experiments at physiological conductivities conditions is advantageous 
for future portable devices because the device/instrument would not need to store dilution buffers. The final results of this project, presented in Chapter 6, explore the entire dielectrophoretic spectra of the ABO-Rh erythrocytes including the cross-over frequency and the magnitudes of the positive or negative dielectrophoretic response. These were completed at lower medium conductivities of $0.1 \mathrm{~S} / \mathrm{m}$ and $0.01-0.04 \mathrm{~S} / \mathrm{m}$. These results show that by using the sweep function built into the Agilent alternating current generator it is possible to explore how a single group of blood cells will react to rapid changes in frequency and will provide the user with curve that can be matched the theoretical dielectrophoretic response curves.

As a whole this project shows that it is possible to distinguish human erythrocytes by their ABO-Rh blood type via three different dielectrophoretic methods. This work builds on the foundation of that it is possible to distinguish healthy from infected cells $\mathrm{s}^{2-7}$, similar cell types ${ }^{1,7-14}$ and other work regarding the dielectrophoresis of human erythrocytes $^{1,10,11}$. This work has implications in both medical diagnostics and future dielectrophoretic work because it has shown that ABO-Rh blood type is now a factor, which must be identified when working with a human blood sample. It also shows that the creation of a microfluidic device that subjects human erythrocytes to a dielectrophoretic impulse and then exports an $\mathrm{ABO}-\mathrm{Rh}$ blood type is a near future possibility. 


\section{INTRODUCTION}

Lab-on-a-chip devices, also known as medical microdevices, are currently being explored for rapid, on-site testing for various medical conditions ${ }^{15}$. Such devices have the potential to perform complicated medical diagnostic tests with a small sample volume, often less than a drop, and do so in a matter of minutes. In roadside emergency situations or in programs such as Doctors Without Borders, devices like this could provide a quantitative means to test for different medical issues when a full medical laboratory is not available. The limited resources and time available in these situations make microdevices that are completely integrated onto a single chip an extremely attractive solution to screen for phenotypes, diseases, and medical issues using quantitative tests instead of simply symptom's diagnosis. The use of only a drop of blood $(<50 \mu \mathrm{L})$ is an advantage in an emergency situation where the patient has already lost a large amount of blood and further blood loss should be prevented. Blood in general is an extremely attractive diagnostic fluid because it provides a plethora of information about all physiological systems in a patient and it is easier to retrieve than other diagnostic materials.

In a traumatic accident or emergency requiring survivor surgery, the hospital needs extra units of compatible blood to replenish substantial blood loss. Right now, patients with unknown blood types are given O- blood because the cell's lack of $\mathrm{ABO}$ antigens prevent most hemolytic reactions. There is thus a large demand for units of $\mathrm{O}$ blood that sometimes is not met by the American Red Cross. However, rapid, point of care testing in the ambulance for the patient's blood type would facilitate usage of other 
compatible blood units. This research project is focused on developing a portable, ABORh blood typing device.

Alternating current dielectrophoresis is a tool with potential in future medical microdevices. Dielectrophoresis is defined as the movement of particles in an inhomogeneous field ${ }^{15}$. Based on the particles intrinsic polarizability, it will experience either positive dielectrophoresis and move towards a region of high field density or negative dielectrophoresis and move towards a region of low field density ${ }^{15}$. Of particular interest in medical microdevice systems is the use of human blood, which many groups have studied ${ }^{1-5,7,9-11,16-25}$. However, our group is the first to test for blood type $\mathrm{e}^{1,10,11}$ and we hope to extend this later to discern abnormalities in blood cells caused by disease. The blood groups in the ABO-Rh typing system differ with respect to the structure of their membrane proteins ${ }^{26}$. The overall HYPOTHESIS of this project is that structural differences in the ABO-Rh antigens on or in the membrane cause a difference in the human erythrocyte's response to an alternating current dielectrophoretic field. This work strives to statistically differentiate between blood types using a small panel of 4 donors for each of the $8 \mathrm{ABO}-\mathrm{Rh}$ blood types.

\subsection{Background: Microdevices, Electrokinetics and Human}

\section{Erythrocytes}

This interdisciplinary project combines best practices, knowledge, and procedures from multiple fields in order to study the electrokinetic response of human erythrocytes. The study platform is a microfluidic device created via soft photolithography discussed in 
further detail in Chapter 4. This PDMS device is loaded, fluid introduced and flow controlled via a microfluidic platform discussed in Chapter 5. Electrokinetic manipulation, specifically dielectrophoresis, is used for erythrocyte manipulation and identification and is discussed in much greater detail in Chapter 3. Human erythrocytes are the studied cell type and are discussed in detail, including information about human blood typing, in Chapter 2. Brief introductions to each of these topics are included in this introduction.

Microdevices, also known as Lab-On-a-Chip (LOC) devices, have generated research interest over the past few decades. Fabrication techniques for microfluidic and microelectronic chips have become more reliable, more accurate and cheaper such that a corresponding advancement has occurred in microfabrication, nanofabrication and microelectromechanical systems (MEMS) techniques. The resulting devices have grown in complexity and capabilities ${ }^{27-4647-7879-107}$. Devices have been developed for uses such as drug screening and delivery ${ }^{88}$, electrochemical detection ${ }^{108}$, environmental monitoring, proteomics and genomics $^{109}$ and medical diagnostics ${ }^{3-5,110}$.

A possible counteraction to the dielectrophoretic force discussed below is the hydrodynamic force, which is dependent on the dimension of the particle, the properties of the suspending medium (viscosity) and the particle's velocity. The particle's velocity is dependent on the mass flow rate and the cross-sectional area of a channel. For microdevices, this can usually be overcome because the electrode spacing allows for voltages to be applied that increase the dielectrophoretic force to be greater than the hydrodynamic. However, problems can arise when a higher throughput is needed which often means increasing the height of the channel without increasing the height 
micropatterned electrodes ${ }^{111}$. For the devices presented in this work this is acknowledged as a potential problem because the height of the channels is on the order of 700 times larger than the height of the micropatterned electrodes presented in Chapters 5 and 6. However, the hydrodynamic drag force has the opportunity to overtake the dielectrophoretic when flow is being induced from either outside the microdevice, such as with the LabSmith Micropumping System, or in another section of the microdevice via pressure-drive or electrokinetically-driven movement of fluid and particles.

In the systems presented in this dissertation all fluid movement was allowed to come to rest before any experiments were started. This allowed the gravitational force to pull the particles down towards the glass/electrode surface of the microdevice and insured that all cells would be exposed to the field. Occasionally fluid movement was seen due to hydrostatic differences in the inlet and outlet reservoirs so these experiments were rerun. Often when this happened, the simplest solution was to even out the liquid level in the inlet and outlet reservoirs, thereby evening their individual hydrostatic pressures and allowing the system to come to equilibrium. When using the LabSmith Micropumping System with the work presented in Chapter 5, this was not as easy to achieve as if the pumping system was not used. This pumping system would add versatility to a microdevice when fluid flow is desired though.

As mentioned above, the force isolated in these experiments is alternating current dielectrophoresis, which belongs to a much larger group of forces known as electrokinetic phenomena. Electrokinetic phenomena are a group of effects that happen when electric fields are applied to conductive fluids including, but not limited to the fields of electrokinetics: electrophoresis, electro-osmosis, diffusiphoresis, capillary 
osmosis, sedimentation potential, streaming potential/current, colloid vibration current and electric sonic amplitude. This section will focus on DC electrophoresis and its counterpart, AC dielectrophoresis.

In classic electrokinetic theory, as a particle surface acquires a net charge, a polarization occurs on the surface and hence around the particle. This polarization causes ions of the opposite charge (counter-ions) to concentrate adjacent to the surface to form what is known as the Debye double layer. Four of the above discussed electrokinetic phenomena exhibit this behavior: electro-osmosis, electrophoresis, streaming potential and sedimentation potential ${ }^{112}$.

Electrophoresis, an electrokinetic phenoma first described by Reuss in 1807, is the movement of dispersed particles in a fluid. This movement occurs because the particles have a surface charge and when an external electric current is applied to the fluid, the magnitude and directionality of this surface charge dictates whether particle will move in the solution to the anode (positive electrode) or cathode (negative electrode or ground). The electric field also exerts a minor force on a layer of diffuse ions of the opposite charge as the particle surface surrounding the particle. This layer is known as the double layer and the force is a retardation force because it is pulling the fluid around the particle in the opposite direction as the particle.

Dielectrophoresis, first defined by Albert Pohl, is the movement of polarizable particles in a non-uniform electric field ${ }^{15}$. The biggest differences between electrophoresis and dielectrophoresis is that for the latter the particle does not need to have an innate charge, just the ability to become polarized, and the field must be spatially non-uniform. This transport phenomena opens up the field of electrokinetics to a much 
wider range of particles because while a good deal of particles, specifically biological cells, aren't charged or are net neutral, they are polarizable. Field non-uniformity is a requirement for DEP and is achieved with an alternating current signal, a direct current signal with an insulating obstacle or some combination of the previous two. This phenomenon will be discussed in much greater detail in Chapter 3. A thorough review of DC dielectrophoresis has been recently published so it will not be discussed in great detail here because it is not the dielectrophoretic force used in these experiments ${ }^{113}$.

Key to the field of dielectrophoresis is dielectric material. Dielectric materials are those materials which when subjected to an electric field can polarize via separation or isolation of their charges. A perfect dielectric would have infinite resistance, making it the perfect insulator. Biological particles are not perfect dielectrics and have four different dielectric parameters that go into the calculation of their final dielectric constant: permittivity of the membrane and cytosol and conductivity of the membrane and cytosol. The ratio of the membrane thickness to the overall radius of the cell also influences the final dielectric constant for the cell ${ }^{6,17,114-118}$. The derivation of the governing equations for dielectrophoresis as well as those for calculating dielectric constants is found in Chapter 3 .

An important factor when dealing with human blood is its $\mathrm{ABO}-\mathrm{Rh}$ type that so this work is focused on the affect of this antigen expression on the erythrocyte's dielectric constant and therefore the cumulative DEP behavior of human erythrocytes. The ABO typing system used for the research proposed herein was discovered by Karl Landsteinner in $1900^{119}$. This typing system is based on the presence or absence of two main antigens, complex carbohydrates found the on the surface of the erythrocytes ${ }^{26,120,121}$. Though the 
number of antigens found on the surface of the erythrocytes can vary from person to person it can be up to as large 1.4 million, which means that small differences in antigen structure can make a large difference in the overall membrane structure ${ }^{122}$. Blood type A is classified by the presence of the A antigen on the cell and the B antibody in the plasma. Blood type B is classified by the presence of the B antigen on the cell and the A antibody in the plasma. Blood type $\mathrm{AB}$ has both antigens and no antibodies and blood type $\mathrm{O}$ has no antigens and both antibodies. Due to this unique antigen-antibody relationship, blood type $\mathrm{AB}$ is often referred to as the universal acceptor and blood type $\mathrm{O}$ as the universal donor $^{26,120,121}$.

In addition to the $\mathrm{A}$ and $\mathrm{B}$ antigens previously discussed a third antigen is critical when matching blood types for procedures such as transfusions ${ }^{26}$. This third antigen is much more complex in nature but is most often referred to as the Rhesus factor. The Rhesus factor is not in fact just a single antigen, but rather a group of antigens. When blood is referred to as a negative blood type it means that the Rhesus factor (more specifically the $\mathrm{D}$ antigen part of the Rhesus factor) is absent and positive blood refers to a case where the Rhesus factor is present ${ }^{121}$. The eight blood types that result from the combination of the $\mathrm{ABO}$ and Rhesus factor systems are: $\mathrm{A}^{+}, \mathrm{A}_{-}^{-}, \mathrm{B}+, \mathrm{B}-, \mathrm{AB}^{+}, \mathrm{AB}-, \mathrm{O}^{+}$ and $\mathrm{O}-$.

The research presented in this dissertation aims to extract the antigen dependence of human erythrocyte behavior under a variety of dielectrophoretic conditions. Work has been done to explore how DEP can be used as a diagnostic tool in separating healthy from infected cells $\mathrm{s}^{2-7}$ and discerning subpopulations of leukocytes ${ }^{12-14}$. Substantial work has even been done using red blood cells, either bovine ${ }^{123,124}$ or human ${ }^{1-5,7,9-11,20,23}$, as the 
cell type of interest in an electrokinetic microdevice. Gascoyne and colleagues ${ }^{3}$ established a method for the removal of leukemia cells from a human blood sample using interdigitated electrodes to trap the leukemia cells at the edges of the electrodes. They've also done work on identifying the differences between erythorcytes infected with Plasmodium falciparum (malaria parasite) and their healthy equivalents, going as far as identifying differences in conductivity and permittivity ${ }^{4,5}$. Alazzam and colleagues designed a continuous flow microfluidic device to separate breast cancer cells from healthy blood cells using the difference between the each cell type's dielectrophoretic and hydrodyanmic forces ${ }^{2}$. Davalos's research group has worked on designing contactless dielectrophoretic device for the separation of cancerous cells from healthy human blood samples $^{7,9,25}$. However, prior to 2008 , only two of these many articles ever noted what blood type was being worked with: $\mathrm{A}+{ }^{23}$ and $\mathrm{O}$ (no Rh factor given) ${ }^{5}$. After 2008, the only articles which made note of blood type when discussing human erythrocytes were those published by this research group ${ }^{1,10,11}$.

\subsection{Motivation}

In 2007, the World Health Organization put together a fact sheet concerning global blood safety and availability of blood for medical purposes. Developed countries account for just $25 \%$ of the world's population, however they account for $65 \%$ of the blood donations. This means that $75 \%$ of the world's population only has access to $35 \%$ of the blood donations given every year, which is far less than they need. At a minimum, $1 \%$ of the country's population must donate blood to meet the basic needs of a country (excluding natural and manmade disasters). In 73 countries worldwide, this $1 \%$ is unmet. 
Of these 73 countries, 71 are classified as developing or transitional. In 2007, 42 countries collected less than $25 \%$ of their blood donations from the safest donation source, which are unpaid and voluntary donations. Thirty-one countries still report collecting paid donations, totaling about 1 million donations. Testing for HIV, Hepatitis B, Hepatitis $\mathrm{C}$ and syphilis (all of which can be transferred during transfusion) is still impossible in 41 countries $^{125}$. These facts show that there is a great need worldwide for safe, continuous donations of blood. If countries are not able to insure that the donor blood is not only disease free but also a compatible blood type to the recipient, any transfusion is risky, potentially yielding further complications for the patient and the medical infrastructure.

The WHO 2007 fact sheet also included data trends regarding the use of donated blood. Transfusions were often given unnecessarily, when simpler and cheaper treatments would suffice. This squandered precious and scarce resources and often led to further complications if the transfusion was not done properly. One hundred and twenty countries comprised of 46 developed countries, 48 transitional countries and 26 developing countries reported a total of 51,400 hospitals performing blood donations to a population of 3.6 billion. Based on data from 96 (38 developed, 40 transitional and 18 developing) of these countries, it was found that:

- A transfusion committee existed in transfusion-ready hospitals in $88 \%$ of developed countries, $33 \%$ of transitional countries and $25 \%$ of developing countries. 
- Mechanisms were in place to monitor clinical transfusion practices in transfusionready hospitals in $90 \%$ of developed, $52 \%$ of transitional and $23 \%$ of developing countries.

- The transfusion-ready hospitals have a system for reporting problems with transfusion events in $91 \%$ of developed, $46 \%$ of transitional and $23 \%$ of developing countries ${ }^{125}$.

The ideal would be to have all three indicators at $100 \%$, in order to protect the patient and prevent further medical costs post-transfusion. One would assume that hospitals in developed countries should have the resources and manpower to adequately monitor all blood transfusions.

With an increased humanitarian emphasis on providing advanced medical care in developing countries through organizations such as Doctors without Borders, there is a need for increased awareness regarding the use and safe handling of blood donations and transfusions. Countries need to increase their population donation rates as well as the percentage of blood donations tested for blood type, blood borne pathogens, and other transfusion-transferrable diseases. The most fundamental aspect of a safe transfusion is to ensure that donor and recipient's ABO-Rh blood types match or are at least compatible. In regions where blood donations are less common, this simple task is more difficult and in an emergency, time-sensitive medical situation, the time it takes to type a patient's blood may be too long. Thus, there is a huge need for an inexpensive, portable, accurate and fast way to type an unknown blood sample. This dissertation describes original research into the design, operation, and data from a medical microdevice that utilizes alternating current dielectrophoresis as an identification tool for ABO-Rh blood type. 


\subsection{Purpose}

The purpose of this project is to optimize operating conditions and study $\mathrm{ABO}-\mathrm{Rh}$ erythrocytes dielectrophoretic responses to alternating current electric signals. The end goal of this project is the creation of a relatively inexpensive microfluidic device, which can be used for the ABO-Rh typing of a blood sample. This dissertation presents results showing how blood samples of a known ABO-Rh blood type exhibit differing behavior to the same electrical stimulus based on their blood type. The first panel of donors and experiments, presented in Chapter 4 occurred when a sample of known blood type was injected into a microdevice with a T-shaped electrode configuration. When subjected to a $1 \mathrm{kHz}$ electrical signal, rupturing of the erythrocytes was observed, analyzed and the responses quantified by blood type, age, washing, buffer, concentration, and frequency. It was determined that the rate at which the cells ruptured over the course of a 15 minute experiment was ABO-Rh blood type dependent. Additionally, other dependencies such as age of the blood sample, electric field strength, washing of erythrocytes with phosphate buffer saline, concentration and frequency were tested to begin to explore how to maximize the rupturing.

The second set of experiments, presented in Chapter 5, were originally published in Electrophoresis in $2011^{1}$. Novel in this work was the discovery that treatment of human erythrocytes with $\beta$-galactosidase successfully removed $\mathrm{ABO}$ surface antigens such that native $\mathrm{A}$ and $\mathrm{B}$ blood no longer agglutinated with the proper antibodies. It was found that cross-over frequency for the blood types tested $(\mathrm{A}+, \mathrm{A}-, \mathrm{B}+, \mathrm{B}-, \mathrm{AB}+, \mathrm{O}+$ and O-) differed over a range of $17 \mathrm{MHz}$ for the native sample, but after treatment with $\beta$ galactosidase this range narrowed to $5 \mathrm{MHz}$ supporting the premise that the $\mathrm{ABO}$ surface 
antigens impact DEP responses. Additionally, this work was performed in a medium conductivity of $0.9 \mathrm{~S} / \mathrm{m}$, which is higher than traditional dielectrophoretic experiment conductivities, but far closer to the conductivity value of pooled plasma at $1.1 \mathrm{~S} / \mathrm{m}$. The ability to perform dielectrophoresis experiments at physiological conductivities conditions is advantageous for future portable devices because the device/instrument would not need to store dilution buffers.

The final results of this project, presented in Chapter 6, explore the entire dielectrophoretic spectra of the $\mathrm{ABO}-\mathrm{Rh}$ erythrocytes including the cross-over frequency and the magnitudes of the positive or negative dielectrophoretic response. These were completed at lower medium conductivities of $0.1 \mathrm{~S} / \mathrm{m}$ and $0.01-0.04 \mathrm{~S} / \mathrm{m}$. These results show that by using the sweep function built into the Agilent alternating current generator it is possible to explore how a single group of blood cells will react to rapid changes in frequency and will provide the user with curve that can be matched the theoretical dielectrophoretic response curves.

This research project has demonstrated that when subjected to an alternating current electrical signal in a non-uniform field human erythrocytes will react differently according to their $\mathrm{ABO}-\mathrm{Rh}$ factor. This was shown in three different setups: a low $\mathrm{kHz}$ region rupturing device (Chapter 4), a typical quadrapole device using frequencies from $1 \mathrm{MHz}$ to $80 \mathrm{MHz}$ and a suspending medium of a physiologically relevant conductivity of $0.9 \mathrm{~S} / \mathrm{m}\left(\right.$ Chapter $\left.5^{1}\right)$ and the same quadrapole device using suspending conductivities between $0.01 \mathrm{~S} / \mathrm{m}$ and $0.1 \mathrm{~S} / \mathrm{m}$ at alternating current frequency sweeps from $10 \mathrm{kHz}$ to $1.9 \mathrm{MHz}$ (Chapter 6). Further, for the last two experimental setups, an enzyme, $\beta-$ galactosidase, was used to cleave the $\mathrm{ABO}$ antigens from the cell surface and 
experimentally show that these antigens affected behavior. This work substantiates the hypothesis that it is possible to distinguish molecular expression using electric fields.

This work has implications on both a theoretical and an experimental level.

Because the human erythrocytes exhibit different polarizability when subjected to the same electrical signal, there must be a factor missing from the equations for the cell's dielectric constant presented in Chapter 3. The state of the equations right now leaves no room for differences on a molecular level. Further theoretical work is needed to match the experimental behavior seen to the current state of dielectrophoretic theory and determine where these missing factors should be included.

Experimentally, this work has broad implications in the area of cell diagnostics. Beginning with the first project regarding erythrocyte lysis with $\mathrm{AC} D E P$, this provides a means to safely lyse cells on a LOC device without adding additional chemical or requiring the high voltages that electroporation often needs. Dielectrophoresis has also been shown to be a convenient way to move fluids so the ability to lyse cells with dielectrophoresis and then move the lysate to a down-chip analysis section is incredibly attractive as chips become more like puzzle pieces ${ }^{126}$.

The work done in the higher $\mathrm{kHz}$ and $\mathrm{MHz}$ regions to determine $\mathrm{ABO}-\mathrm{Rh}$ affects on dielectrophoretic behavior also have widespread implications in cell diagnostics. The most obvious of these is that eventually it will be possible to have a medical microdevice that tests for blood type. The initial results presented in this dissertation show that the differences in erythrocyte response to an AC signal are already significant enough that certain blood types can be distinguished from others. Further experiments with a larger donor population will only help enhance these differences so that the exact tests that need 
to be run to output a specific ABO-Rh blood type becomes evident. The work presented here not only forms the baseline for a blood typing device but also provides the starting point for further DEP analysis using erythrocytes. Although work has been published involving the separation of unhealthy cells from human blood, this field is by no means extensive. Blood is the most readily available diagnostic fluid and if the molecular differences from $\mathrm{ABO}-\mathrm{Rh}$ antigen expression are enough to change dielectrophoretic responses, then other molecular level changes could be detected using similar methods. The first step for this is obviously to know the ABO-Rh blood type of the blood sample of interest because then the deviations from this characteristic curve can be tied to molecular level changes or morphological changes that indicate diseases such as sickle cell anemia. 


\section{Human Blood System: Biochemistry and Electrokinetic}

\section{Manipulation}

\subsection{Introduction}

Blood is a convenient and informative bodily fluid that carries chemical signatures from all of the human systems, which is why it is often used for medical diagnostics and research. The following chapter reviews the whole blood system, with a special emphasis on human erythrocytes and the 30 major blood typing systems ${ }^{127}$. Concurrently, this work compiles from a biochemical perspective what is known about the human blood systems (specifically, erythrocytes and leukocytes) in order to link this body of knowledge to the dielectric properties we measure experimentally.

Dielectrophoresis has been widely explored to separate, manipulate, and identify bioparticles ${ }^{128}$ by harnessing micrometer or nanometer scale movements in microdevices $^{129}$. The technique has been applied to many studies on erythrocytes ${ }^{1-5,7,9-}$ 11,20,23,124 including removing leukemia cells from blood ${ }^{3}$ and separating blood cells infected with the malaria parasite from unharmed cells ${ }^{4,5}$. Alternating current dielectrophoresis is defined as the movement of particles in an inhomogeneous field. Based on the particle's intrinsic polarizability, it will experience either positive dielectrophoresis and move towards a region of high field density or negative dielectrophoresis and move towards a region of low field density ${ }^{15}$. The intrinsic polarizability of a cell is made up of a combination of the permittivity and conductivity of 
both its membrane and cytosol, which are the dielectric properties of the cell. As either of these components change biochemically, the intrinsic polarizibility also changes. This allows for the precise manipulation of cells with minor biochemical differences.

Comparisons will be drawn to other blood cell types due to the limited availability of healthy erythrocyte knowledge. A body of work has been published on the dielectrophoretic behaviors of subpopulations of leukocytes ${ }^{12-14,129}$, tumorous cells separated from blood ${ }^{7,9}$ and malarial infected erythrocytes ${ }^{4,5}$. Hence, compiling the known biochemical structure of these cell types and correlating with the reported dielectrophoretic responses of these same cells will increase the scientific usefulness of both bodies of knowledge. Dielectrophoretic theory can be altered to correlate observations with cell phenotype and stage while the biological systems will have a new tool with which to characterize physical properties.

This review focuses primarily on erythrocytes because although a body of literature exists for this complex cell type, almost all dielectrophoretic characterizations have largely ignored how the biochemical phenotypes of the erythrocyte affect the electrokinetic response. Of particular interest are antigen phenotypes, especially those antigens involved in blood typing. It has been determined by the authors that the expression of $\mathrm{ABO}-\mathrm{Rh}$ antigens on the human erythrocyte does change its dielectrophoretic behavior and polarizability ${ }^{1}$.

\subsection{Human Blood System}

The human blood system is comprised of four main parts, each with its own function and characteristics. These are erythrocytes (red blood cells), leukocytes (white blood cells), thrombocytes (platelets) and plasma. Plasma is the highly conductive 
suspending fluid for the human blood system. When fibrinogen and other clotting factors are removed, this fluid is referred to as serum. This fluid carries antibodies, electrolytes, hormones, as well as complex and diverse chemicals for signaling, nutrient delivery, and system homeostatis throughout the body ${ }^{130}$. Thrombocytes are cell fragments, which are on the order of $2 \mu \mathrm{m}$ in diameter. Their function is aid in the body's clotting mechanism ${ }^{130}$. Leukocytes (lymphocytes, basophils, neutrophils, macrophages, etc) vary greatly based on their subset in both shape and size, but range from 3.09 to $4.19 \mu \mathrm{m}$. Their function is to provide a defense mechanism for the body against foreign invaders and to dispose of the body's innate cells that have become old or compromised. Erythrocytes are biconcave disks on the order of $6-8 \mu \mathrm{m}$ in diameter. Their primary function is to transport oxygen and carbon dioxide throughout the body ${ }^{26,131}$. These blood components work in concert to transport necessary nutrients and maintain the health of all of the human body's systems.

\subsubsection{Cell origins}

Three of the four main components (thrombocytes, leukocytes and erythrocytes) of blood are all derived from the same progenitor cell, the pluripotent hematopoietic stem cell through a process called hematopoiesis. This process takes place mainly in the human bone marrow. In its first stage of differentiation, the hemtopoietic stem cell divides into three different progenitor cells: lymphoid, myeloid and erythroid. The erythroid progenitor cells eventually develop into erythrocytes and megakaryocytes, which are the cells that produce thrombocytes. The myeloid and lymphoid progenitor

cells develop into the myriad of cells commonly referred to as leukocytes ${ }^{132}$. These will 
be discussed in greater detail in Section 2.2.4. A full outline of the cells derived from the hematopoietic stem cell is shown in Figure 2.1.

\subsubsection{Plasma}

Blood plasma is the fluid that transports the erythrocytes, leukocytes and platelets around the body. It is a highly conductive medium comprised mainly of proteins, electrolytes and organic molecules ${ }^{131}$. Typically the blood plasma makes up $55 \%$ of the total volume of whole blood and $91.5 \%$ of this is water. The other big component of the plasma are the proteins, which comprise $7 \%$ of the plasma volume. The plasma proteins comprise mostly of albumins (54\%), globulins (38\%) and fibrogen (7\%). The final component of the blood plasma are other random solutes that the body needs to transport, such as electrolytes, nutrients, gases, waste and other regulatory molecules ${ }^{130}$.

Each of the plasma constituents has its own function and purpose in the blood system. The water is there to act as a suspending medium and assist in the transfer of heat. Albumin transports both steroid hormones and fatty acids through the body. It is also the smallest of the plasma proteins. Globulins are produced by the liver and the alpha and beta versions transport iron, lipids and any vitamins that are fat soluble. They also have complimentary proteins that are known as immunoglobulins and help with the body's defenses against bacteria and viruses. Fibrogen's main role in the blood system is to assist in the body's clotting mechanism. The plasma also carries electrolytes such as $\mathrm{Na}^{+}, \mathrm{K}^{+}, \mathrm{Ca}^{2+}, \mathrm{Cl}^{-}$and $\mathrm{SO}_{4}{ }^{2-}$. These electrolytes maintain the cells osmotic pressure and also serves in various functions within a cell. The nutrients carried by the plasma can include things such as amino acids, glucose, vitamins and minerals. The most common 
gas in the plasma is $\mathrm{CO}_{2}$ in its dissolved state because $\mathrm{O}_{2}$ is most often found bound to the erythrocyte's hemoglobin. The waste in the plasma is there to be carried to other parts of the body such as the liver for excretion from the body. The regulatory molecules keep the body working properly and include substances such as enzymes, hormones and $\operatorname{vitamins}^{130}$.

In work published by Visser et al, they developed an equation to calculate the approximate conductivity of a blood sample. As the hematocrit level decreases, the conductivity of the solution increases to a pure plasma limit of $1.57 \mathrm{~S} / \mathrm{m}^{133}$. Pooled plasma from several donors in a study by the authors was measured as $1.1 \mathrm{~S} / \mathrm{m}^{1}$. In most experimental studies, cells are separated from the plasma in order to more tightly control the suspending medium. However, performance of blood tests in situ would reduce sample preparation time and cost. Current blood typing techniques require erythrocyte separation from the suspending plasma in order to mix with a purified antibody array to test for agglutination and therefore blood type.

\subsubsection{Thrombocytes}

Platelets are the smallest of all the blood cells $(2 \mu \mathrm{m})$ and are disc-shaped with no nucleus but a fairly complex internal structure. They are formed when megakaryoblasts change into megakaryocytes which then fragment into between $2000-3000$ pieces, each

of which becomes a platelet ${ }^{130}$. Platelets maintain blood vessel integrity by initiating and participating in clotting to prevent blood loss ${ }^{132}$. In whole blood samples, the platelet count is between 150 to $400 \times 10^{9}$ cells per microliter which is a single order of magnitude larger than that of leukocytes ${ }^{131}$. Platelets have the primary function of 
stopping blood loss in damaged blood vessels by forming a plug. They have an incredibly short life span of only 5-9 days ${ }^{130}$ and are not often studied.

To the author's knowledge, the dielectric properties of platelets have not been measured. Since the dielectrophoretic force is proportional to the volume of the cell, these measurements will require much higher electric fields and will experience more noise than characterizations of erythrocytes or leukocytes. Most work done with blood samples specifically targets either the leukocytes or the erythrocytes because they exist is greater quantities and have biochemical functions that are of general scientific interest.

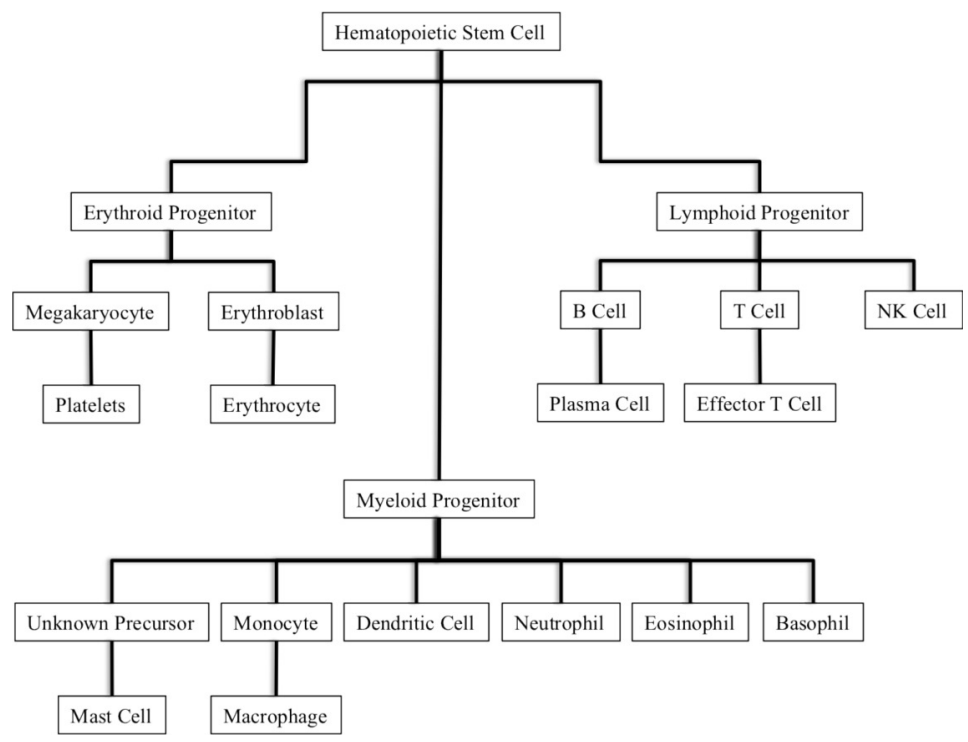

Figure 2.1. Blood and Tissue Cells derived from a common Hematopoietic stem cell. Information from ${ }^{132}$.

\subsubsection{Leukocytes (White Blood Cells)}

Leukocytes are the least numerous of all the cells in the human blood system, about $5 \times 10^{3}$ cells per microliter of blood, which is 3 orders of magnitude less than erythrocytes $^{131}$. The healthy range of leukocytes is $5000-10000$ cells $/ \mu 1^{130}$. Unlike erythrocytes, which are largely homogenous in size, shape and functionality, leukocytes consist of a variety of subpopulations. The main function of leukocytes in the human 
blood system is to fight infection, however the mechanism by which they do this varies based on their subclass as is tabulated in Table 2.1. They are the body's first line of defense whenever a foreign agent of any sort is introduced to the blood system.

Leukocyte subpopulation types are specialized for the foreign agent they target and have optimized attack mode as well ${ }^{132}$. The five main subclasses of leukocytes found in the blood stream and their percentage of abundance are: neutrophils (40-75\%), eosinophils (1-6\%), basophils $(<1 \%)$, monocytes $(2-10 \%)$ and lymphocytes $(20-50 \%)^{132,134}$. Each of these subpopulations has its own function, which is described briefly in Table 2.1 and in greater detail below.

Table 2.1.

Types and functions of leukocytes. Information from ${ }^{132}$.

\begin{tabular}{|c|c|}
\hline Leukocyte Cell Name & Primary Function ${ }^{132}$ \\
\hline Small lymphocyte & $\begin{array}{l}\text { Produces antibodies (B cells) and cytotoxic or helper functions ( } \mathrm{T} \\
\text { cells) }\end{array}$ \\
\hline Plasma cell & B cells that secretes antibodies \\
\hline $\begin{array}{l}\text { Natural killer cell (large } \\
\text { granular lymphocytes) }\end{array}$ & Killer cells that are infected with a virus \\
\hline Neutrophil & Phagocytosis and microorganism killing \\
\hline Eosinophil & Releases granule contents to kill antibody-coated parasites \\
\hline Basophil & Unknown function \\
\hline Dendritic cell & Activates T-cells and initiates the adaptive immune response \\
\hline Mast cell & $\begin{array}{l}\text { Expels parasites from body by releasing granules that contain } \\
\text { agents such as histamine }\end{array}$ \\
\hline Monocyte & Precursor cell to macrophage that circulates in blood stream \\
\hline Macrophage & $\begin{array}{l}\text { Phagocytosis and microorganism killing as well as activation of } \mathrm{T} \\
\text { cells and initiation of immune response }\end{array}$ \\
\hline
\end{tabular}

One of the subsets of leukocytes is the granulocytes, which release granules into the cytoplasm to kill microorganisms. These granulocytes are also called polymorphonuclear leukocytes because they have an irregularly shaped nuclei with anywhere from two to five knobs. These granulocytes are cells dervice from the myeloid progenitor, as seen in Figure 2.1. In order of abundance, these polymorphonuclear leukocytes are: neutrophils, eosinophils and basophils. Neutrophils are the most lethal. 
They rapidly organize, enter the site of infection, and have the ability to work in anaerobic conditions. Eosinophils defend against worms and intestinal parasites. The basophils are rare and little is known about their contribution to the immune system. These cells are historically named based on granule staining in histological tests. The eosinophil's granules are basic and bind the acidic stain eosin whereas the basophil's granules are acidic and bind a basic stain such as hematoxylin. The neutrophil's granules are neutral and bind neither acid or basic stains ${ }^{132,134}$.

A second set of leukocytes is derived (Figure 2.1) from the lymphoid progenitor, the large granular lymphocytes and the small lymphocytes. The large granular lymphocytes are also known as natural killer cells and defend the body against viral infection. Working primarily in the tissue, they prevent the spread of infection by killing virus-infected cells and excreting cytokines that prohibit further viral replication. The small lymphocytes are B-lymphocytes and T-lymphocytes and are primarily responsible for the adaptive immune response. They typically circulate in an inactive, immature form. When the body detects a foreign pathogen, the small lymphocytes differentiate to fight the invading microorganism over the course of 1-2 weeks. These differentiated cells differ only by the cell-surface receptors, which are immunoglobulins and T-cell receptor, respectively. Each lymphocyte will express a single type of immunoglobulin or T-cell receptor specific to the pathogen detected by the immune system ${ }^{132}$.

\subsubsection{Biochemical and Electrokinetic Analysis}

Differences between leukocytes subtypes are traditionally explored via histological stains. Histological staining is used to identify lymphoid leukocytes subpopulations based on granule staining ${ }^{132}$. Diagnostic information is limited by 
microscope resolution; at lower resolutions, the entire leukocyte will appear stained such that granules number and position is lost. However, Vykoukal and colleagues successfully utilized dielectrophoresis to characterize leukocyte cell types. As seen in Figure 2.2, appreciable differences in cross over frequency exist between the different subpolymorphonuclear leukocytes. By plotting the crossover frequency versus medium conductivity, the specific membrane capacitance for each cell type was calculated from the slope of the plotted line. These differences in membrane capacitance combined with leukocyte size variations could be exploited with dielectrophoresis and electrokinetics to separate the 6 leukocyte subpopulations without centrifugation. The conclusion made was that an impedance-based approach to leukocyte cell identification and separation is feasible $^{12}$, however no conclusions were drawn in regards to the biophysical reasoning behind why such closely related cell types would have such discernable differences in their membrane capacitance. 


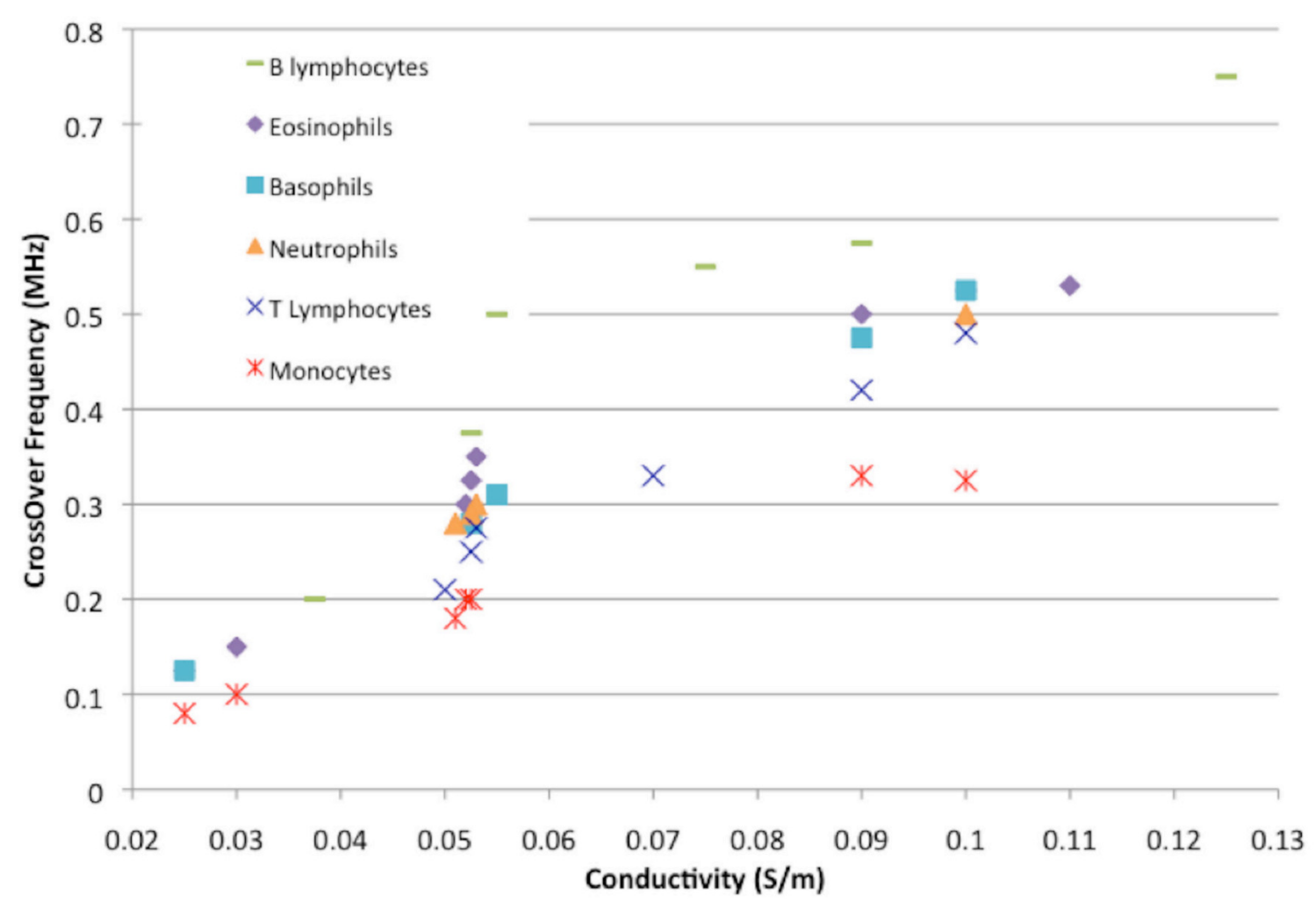

Figure 2.2. Graphical representation of the differences in dielectrophoretic crossover frequency (in MHz) based on medium conductivity and the following subpolymorphonuclear leukocytes: B lymphocytes (-), Eosinophils (u), Basophils (n), Neutrophils ( $\square$, T lymphocytes $(\times)$, and Monocytes $\left({ }^{*}\right)$. Data taken from: Vykoukal, D. M., Gascoyne, P. R. C., Vykoukal, J., Integrative Biology 2009, 1, 477-484.

More recently, a group used DEP field flow fractionation (FFF) to separate mixtures of these leukocyte populations. Based on the different polarizabilities of the subpopulation cells, they will experience different DEP levitation forces and as the fluid is pushed through the device, they will elute out of the device at different times. When a device such as this one is attached to a flow cytometer then the elution peaks can be recognized and corresponded to different cell types. The separation of lymphocytes from monocytes, lymphocytes from granulocytes and monocytes from granulocytes was explored. When lymphocytes were separated from monocytes, the first elution peak was shown to be $92 \%$ T-lymphocytes and the second was $98 \%$ monocytes. The separation between T-lymphocytes and granulocytes showed a first peak of 87\% T-lymphocytes and 
$94 \%$ granulocytes. The final separation had a first peak of $91 \%$ granulocytes and $97 \%$ granulocytes $^{13}$. For cell types that are so closely related biochemically this was a substantial separation. The basis for separation was attributed not to differences in membrane protein-lipid ratios but rather to the differences in morphology that had been previously explored by the same group ${ }^{14}$.

It was shown through electrorotation studies that there were discernable differences in membrane capacitance between T-lymphocytes $\left(10.5 \pm 3.1 \mathrm{mF} / \mathrm{m}^{2}\right)$, Blymphocytes $\left(12.6 \pm 3.5 \mathrm{mF} / \mathrm{m}^{2}\right)$, monocytes $\left(15.3 \pm 4.3 \mathrm{mF} / \mathrm{m}^{2}\right)$ and granulocytes $\left(11.0 \pm 3.2 \mathrm{mF} / \mathrm{m}^{2}\right)$. SEM images in Figure 2.3 were used to see the morphological differences between the four cell types tested and then the authors tried to make connections between the measured differences in membrane capacitance and the morphological differences ${ }^{14}$. Even between T-lymphocytes and B-lymphocytes, which are about the same size, there are surface morphology differences. The T-lymphocytes have a mostly smooth surface, while the B-lymphocytes have microvilli of length 0.1 to $0.6 \mu \mathrm{m}$ and width $0.1 \mu \mathrm{m}$. Also learned from SEM images was that there are large differences in surface morphology within a given cell population, which manifested itself in the electrorotation experiments as large standard deviations in membrane capacitance $\left(3.1-4.3 \mathrm{mF} / \mathrm{m}^{2}\right)^{14}$. This and the follow-up paper are the only papers found by the author that indicate a direct correlation between a typical biological test (SEM imaging) and DEP/ROT tests to explain how the morphology of a cell directly affects its dielectric properties ${ }^{13,14}$. Further work needs to be done to bridge the gap between these two areas so that what is learned from DEP can be correlated to what is known about cells 
biologically and then this correlation can be applied to cell's whos function and morphology are not yet as well known as leukocytes and erythrocytes.
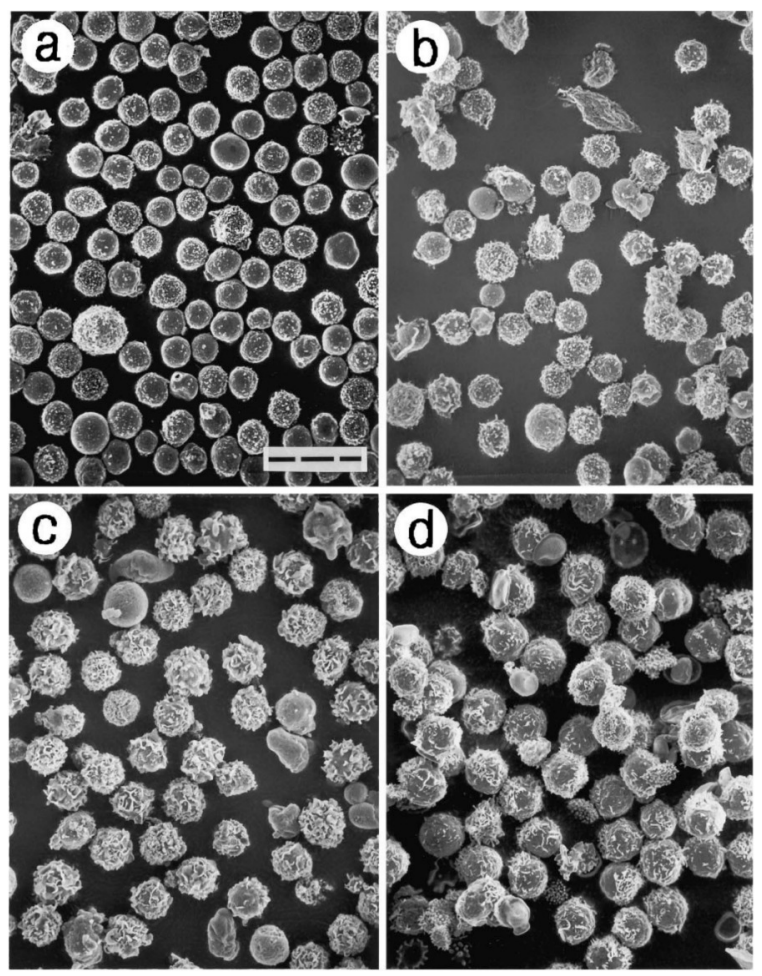

Figure 2.3. SEM Images of T-lymphocytes (A), B-lymphocytes (B), Monocytes (C) and Granulocytes (D). The scale bar corresponds to $20 \mu \mathrm{m}$. Reprinted with permission from ${ }^{14}$.

\subsubsection{Erythrocytes (Red Blood Cells)}

Red blood cells are biconcave disks of diameter 6-8 microns and thickness 2

microns near the outside and 0.5 microns in the center; this shape maximizes the surface through which the cells can transport oxygen and carbon dioxide. A normal cell has a volume of $90 \mathrm{fL}$ and a surface area of $140 \mu \mathrm{m}^{2}$, an excess of $40 \%$ surface area over a sphere of the same dimensions ${ }^{135}$. The life span of a red blood cell in circulation is approximately 120 days and undergoes physiological changes during that time including increased protein density, and increased membrane fragility. Membrane fragility can 
make red blood cells more susceptible to hemolysis ${ }^{26}$. The flexibility of the membrane allows the cells to travel quickly through the blood stream and to travel into even the smallest blood vesicles.

The average adult has 4.2 to $6.2^{*} 10^{6}$ red blood cells per microliter. For males, the average is 5.5 million red blood cells per microliter as opposed to 4.5 million red blood cells per microliter for females ${ }^{26}$. This corresponds to healthy hematocrit levels of 40.7$50.3 \%$ for males and $36.1-44.3 \%$ for females.

The erythrocyte membrane is a lipid bilayer supported by spectrin dimers crisscrossing inside the cell. Erythrocyte membrane composition is $50 \%$ protein, $10 \%$ carbohydrate (both glycoproteins and glycolipids) and 40\% lipid ${ }^{131}$. Figure 2.4 shows the chemical structures of the primary lipids comprising the lipid bilayer, the spectrin, and proteins traversing the lipid layer. This unique structure was first discovered by Gorter and Grendel in 1925, where they showed that chromocytes (any pigmented blood cell) had a bilipid membrane with the polar groups oriented to the inside and outside of the cell ${ }^{136}$. In the last 90 years much knowledge has been accumulated on erythrocyte membrane composition, function, and structure.

For human erythrocytes, the membrane is an essential and dynamic barrier with diverse functionality due to antigen expression, transport and any mechanical characteristics $^{135}$. Spectrin provides both support and flexibility for the membrane by acting much like a membrane skeleton as shown in Figure 2.4. The spectrin dimer was extracted from erythrocyte ghosts and isolated into a single band on a polyacrylamide gel meaning that it is homogenous in terms of its protein expression. Marchesi and colleagues in 1968 thoroughly tested the spectrin protein, which makes up about $20 \%$ of 
the membrane-bound protein of a human erythrocyte, and concluded it was involved in maintenance of the membrane structure ${ }^{137}$. In 1971, Steck and colleagues isolate the interior and exterior faces of the membrane and determined the proteins were not arranged symmetrically and that many proteins span the entire membrane ${ }^{138}$. In the outer lipid leaflet layer, sphingomyelin, cholesterol and phosphatidylcholine are prevalent while in the inner lipid leaflet layer as shown in Figure 2.4. Singer and colleagues then proposed a fluid mosaic model of the cell membrane, with the proteins freely floating within the lipid bilayer with the capability of moving as needed ${ }^{139}$. Figure 2.4 intends to depict these attributes of the erythrocyte membrane. The membrane fluidity combined with the spectrin skeleton facilitates incredible flexibility. An erythrocyte can withstand up to $250 \%$ linear extension but a mere $3-4 \%$ increase in cell volume will result in lysis ${ }^{135}$.

The cytosol is the suspending medium inside the human erythrocyte. It has a high ion concentration, which results in a conductivity much higher than that of the erythrocyte membrane ${ }^{20}$ and usually higher than the medium in which erythrocytes are suspended for dielectrophoretic experiments ${ }^{3,4,140}$. This group, however, has done work at a medium suspension of $0.9 \mathrm{~S} / \mathrm{m}$ which is close to the pure plasma calculated conductivity of $1.57 \mathrm{~S} / \mathrm{m}^{141}$, in order to determine the dielectrophoretic behavior and the dielectric properties of human erythrocytes in a physiologically relevant state ${ }^{1}$. 


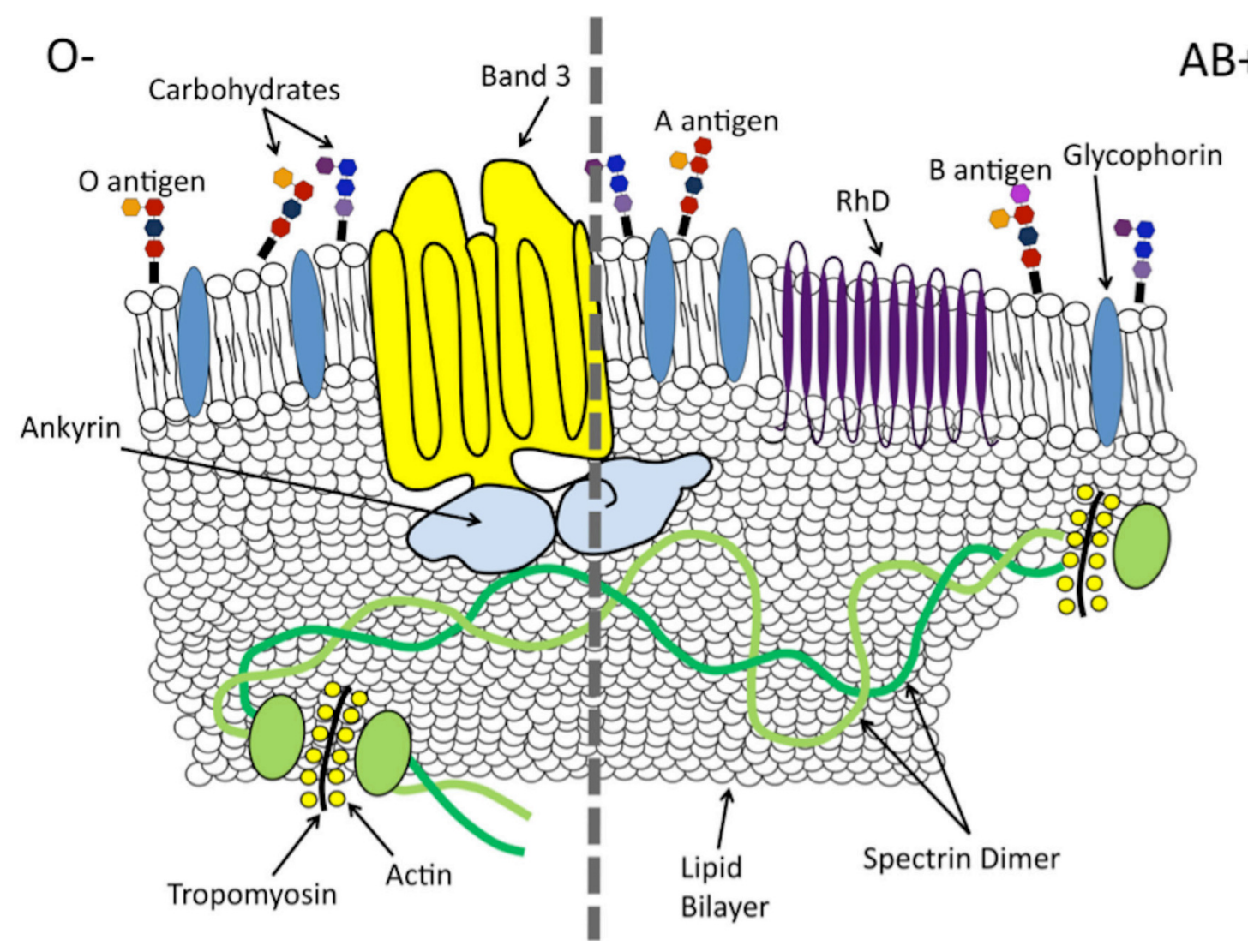

Figure 2.4. Major proteins and antigens of the human erythrocyte membrane. On the left is $O-$-, showing the $O$ antigen and lack of Rhesus factor. On the right is $A B+$ showing both the $A$ and $B$ antigens and the transmembrane Rhesus factor. Also drawn is the spectrin dimer, which provides support and stability for the membrane.

\subsubsection{Human Blood Typing: Antigens and Antibodies}

An incredibly important factor when working with blood is the blood type. There are currently 30 systems accepted by the International Society of Blood Transfusions for defining the type of a particular blood sample ${ }^{127}$. Each system has a particular set of antigens whose presence or absence determines blood type. The two most common systems are those defined by the $\mathrm{ABO}$ antigens and the Rhesus group; the $\mathrm{ABO}-\mathrm{Rh}$ typing system is the primary antigen/antibody system dominating blood compatibility for blood transfusions. Hence, the ABO-Rh blood type is widely documented in medical 
records and American Red Cross donations. The other blood typing systems are briefly discussed below.

The ABO antigens are polysaccharide molecules, or complex carbohydrate antigens found on the cell membrane. Type A blood cells express the A antigen and type $\mathrm{B}$ blood cells express the $\mathrm{B}$ antigen. Type $\mathrm{AB}$ blood has both $\mathrm{A}$ and $\mathrm{B}$ antigens and type O blood has neither antigen ${ }^{26}$. In addition to the $\mathrm{A}$ and $\mathrm{B}$ antigens, there is a third antigen, known as the Rhesus factor, that is considered in matching blood types during transfusions ${ }^{26}$. If the $\mathrm{Rh}$ antigen is present, the blood type is classified as positive and if it is absent the blood type is positive ${ }^{120}$. Therefore, the eight resulting blood types are A+, $\mathrm{B}+, \mathrm{O}+, \mathrm{AB}+, \mathrm{A}-, \mathrm{B}-, \mathrm{O}-, \mathrm{AB}-$.

The ABO blood typing system was established by Landsteiner in $1900^{119}$ when he discovered blood cells of some individuals agglutinated when mixed with the blood plasma from other individuals. Agglutination is the process by which an antibody attaches to its corresponding antigen on more than one cell and each cell has more than one antibody attached to it, resulting in a clustering effect. Landsteiner earned a Nobel Prize by making note of when agglutination did and did not occur and discovering that blood could be broken up into different groups. During Landsteiner's exploration of what became known as the $\mathrm{ABO}$ typing system, he discovered that erythrocytes expressing one antigen would react with the plasma from a person with erythrocytes that did not express that same antigen. He named the first two antigens discovered A and B. He also realized that some blood cells do not express either antigen and therefore react with all plasmas and this became known as blood type $\mathrm{O}$. The year after this discovery, blood type $\mathrm{AB}$ was added to the system when it was discovered that some erythrocytes 
did not react to any plasma ${ }^{142}$. Therefore type A blood is classified by the presence of the $\mathrm{A}$ antigen and type $\mathrm{B}$ blood is classified by the presence of the $\mathrm{B}$ antigen. Type $\mathrm{AB}$ blood has both $\mathrm{A}$ and $\mathrm{B}$ antigens and type $\mathrm{O}$ blood has neither antigen ${ }^{26}$. The two characteristic antigens of the ABO system vary by a single substitution on the terminal end of the carbohydrate antigen. Antigen A has N-acetyl-D-galactosamine, whereas B has $\mathrm{N}$-acetyl-D-glucosamine. The full carbohydrate structures of the $\mathrm{A}, \mathrm{B}$ and $\mathrm{O}$ antigens can be seen in Figure 2.4. Since the number of antigens per erythrocyte can be as high as 1.5 million this small change in chemical structure makes a large cellular difference ${ }^{122}$.

The reactant present or absent in the plasma was determined to be powerful antibodies against the given antigens. The presence of these anti-A and anti-B antibodies differs from normal immunological function. Normally the body produces antibodies upon exposure to the antigen; the AB blood type antigen is an exception. The IgG and IgM antibodies that are associated with the ABO blood typing system are naturally occurring, meaning that a person who has blood type A will naturally produce the anti-B antibodies and the a person with blood type B will naturally produce the anti-A antibodies. When the A antigen comes into contact with a plasma containing the Anti-A antibody this antigen-antibody complex will occur causing agglutination. The AB antibodies exist because they are central to cell hemolysis in vivo. AB antibody-protein complexes bind to the active site on the erythrocyte and start the complement cascade, which will lyse the cell while it is still in circulation (otherwise known as intravascular hemolysis $)^{142}$. An acute hemolytic transfusion reaction occurs when a patient is transfused an incompatible blood type; this agglutination mechanism is the underlying cause of most ABO-incompatible transfusion deaths ${ }^{142}$. The other most common disease 
associated with the $\mathrm{ABO}$ antigen-antibody complex is hemolytic disease of the newborn (HDN). This disease occurs with fetuses of blood type A or B with a type O mother.

Anti-A and anti-B in the mother's blood are of type IgG, which can cross the placenta.

This can cause complications for the fetus, but HDN tends to be mild since fetal erythrocytes express low levels of $\mathrm{A}$ and $\mathrm{B}$ antigens ${ }^{142}$.

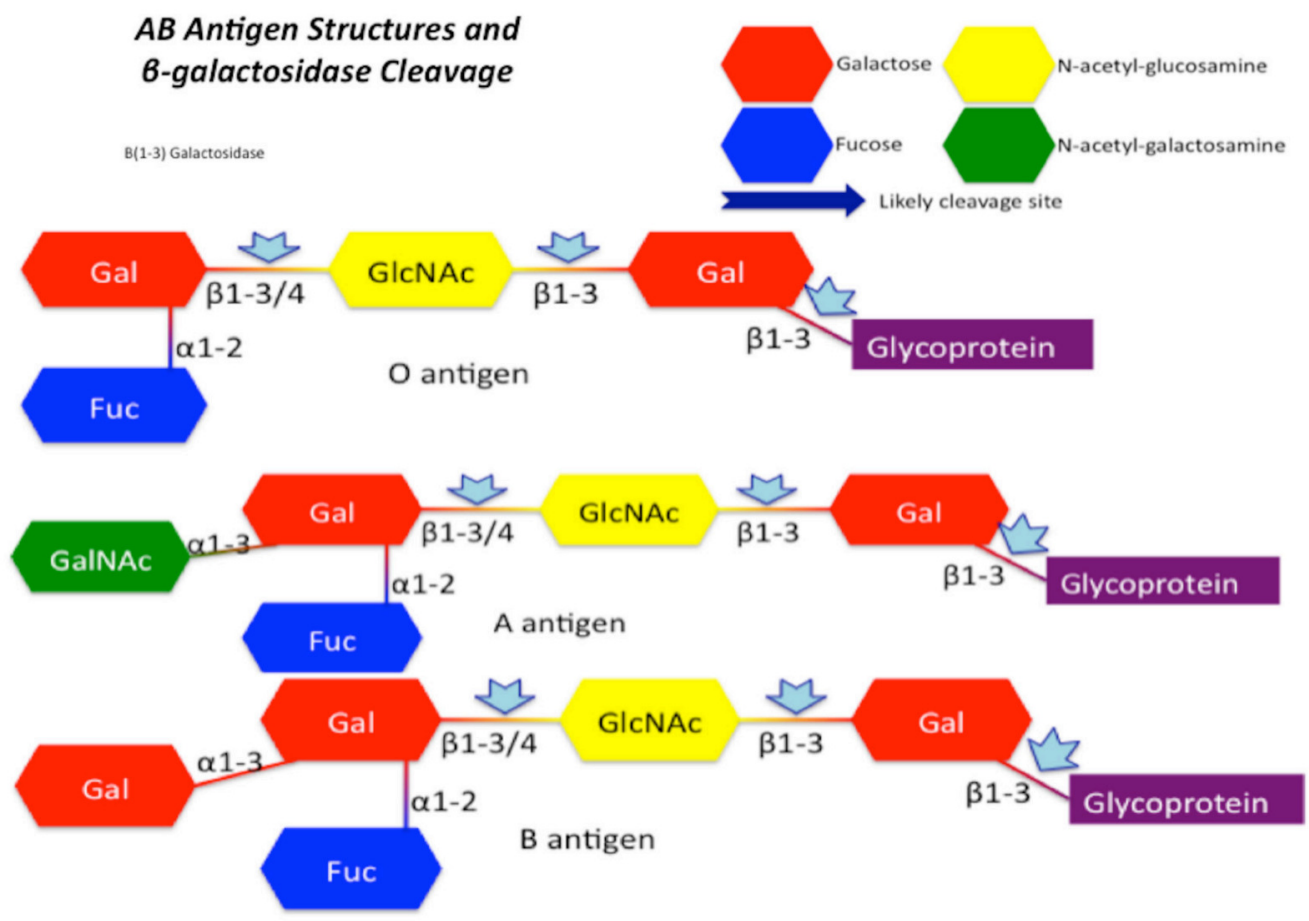

Figure 2.5. Carbohydrate structures of $\mathrm{O}, \mathrm{A}$ and $\mathrm{B}$ antigens as they appear on a human erythrocyte. The glycoprotein attaches the carbohydrate structure to the cell membrane ${ }^{120,121}$.

While commonly referred to as a single antigen, the Rhesus factor is actually a group of transmembrane proteins that function together. The presence of the D antigen portion of the Rhesus factor group it is classified as Rh positive and blood is classified as Rh negative if the $\mathrm{D}$ antigen is not present ${ }^{26,120,121,131}$. Evidence exists that this class of transmembrane proteins function as ammonium transporters (AMTs) because of the 
homological similarity of the RHAG to the animal erythrocyte equivalent of an AMT ${ }^{143}$ Blood is classified as positive if it expresses RHAG and negative if it does not ${ }^{120,121}$.

There are 28 other blood typing systems in addition to the ABO and Rhesus factor systems for the purposes of typing a human donor's blood. Table 2.2 summarizes the epitope information for all 30 blood typing systems currently accepted by the ISBT, which contains the type and name of antigens used to characterize the system. A given human blood sample can be categorized in each of the systems although $\mathrm{ABO}-\mathrm{Rh}$ is most commonly used for matching blood types for transfusions. This means that two blood samples may share the same ABO-Rh type, but the erythrocytes differ by other antigens. For example, two A+ blood donors could be $\mathrm{Le}^{\mathrm{a}}$ and $\mathrm{Le}^{\mathrm{b}}$, respectively according to the Lewis blood typing system. From the electrokinetic context, erythrocyte differences between two donors of the same ABO-Rh blood type could be due to other antigen-level differences. 
Table 2.2.

International Society of Blood Transfusions 30 recognized blood typing systems and their corresponding epitopes. Information from ${ }^{127}$.

\begin{tabular}{ll}
\hline System Name & Epitope \\
\hline ABO & Carbohydrate. Antigens A, B \& H. \\
MNS & Glycophorins A \& B. Main antigens: M, N, S, s \\
\hline P & Glycolipid. Antigen P1 \\
Rh & Protein. C, c, D, E and e. \\
Lutheran & Protein. Set of 21 antigens \\
Kell & Glycoprotein. K1 \\
Lewis & Carbohydrate. Main antigens: Le and Le \\
\hline Duffy & Protein (chemokine receptor). Main antigens: Fy and Fy ${ }^{\mathrm{b}}$ \\
\hline Kidd & Protein. Main antigen Jk and Jk \\
\hline Diego & Glycoprotein. \\
Yt or Cartwright & Protein. \\
XG & Glycoprotein \\
Scianna & Glycoprotein \\
Dombrock & Glycoprotein \\
Colton & Aquaporin1. Main antigens: Co(a) and Co(b) \\
Landsteinner-Wiener & Protein (immunoglobulin superfamily) \\
Chido/Rodgers & C4A and C4B \\
Hh/Bombay & Carbohydrate \\
Kx & Glycoprotein \\
Gerbich & GPC/GPD (Glycophorins C and D) \\
Cromer & Glycoprotein (DAF or CD55) \\
Knops & Glycoprotein (CR1 or CD35) \\
Indian & Glycoprotein (CD44) \\
Ok & Glycoprotein (CD147) \\
Ralph & Transmembrane glycoprotein \\
JMH & Protein \\
\hline Ii & Branched (I)/ Unbranched (i) polysaccharide \\
Globoside & Glycolipid. Antigen P \\
GIL & Aquaporin 3 \\
Rh-associated & Rh-associated glycoprotein \\
glycoprotein & \\
\hline & \\
\hline
\end{tabular}

\subsubsection{Biochemical and Electrokinetic Analysis}

Typically, the structure and function of human blood cells are explored via microscopic and biochemical analysis methods. The most common method of blood typing also happens to be one of the most common methods of cellular analysis in general. There is a wide area of rapid antigen detection tests based on the idea that an 
antigen will bind to its corresponding antibody. The most common of these tests, and the one used for $\mathrm{ABO}$ blood typing, is an agglutination test. In a typical agglutination test, particles coated with the antibody of interest are placed on a glass slide and then the specimen of interest is added and the sample is mixed. The test is read visually by searching for clusters of the cells of interest. Generally these tests are qualitative in nature but can be made semi-quantitative by changing the dilution of the cell of interest and noting the dilution that produces the minimum or maximum reaction. A false negative occurs as a result of "prozoning", which is where there is an over abundance of the antigen and therefore the antibodies will adhere to only one antigen and the clumping that is indicative of agglutination is prevented. This result can be checked by redoing the test at a higher dilution of the antigen. This type of rapid antigen detection tests tends to be extremely fast and requires little equipment or training, however it also is not as sensitive as other tests such as enzyme immunoassays or fluorescent detection ${ }^{144}$. It is this agglutination test for ABO blood typing that the work described within this document is aiming to replace in order to avoid the false negative results that can often occur as a result of the test being read incorrectly.

The other two main forms of rapid antigen detection tests are immunofluorescence and enzyme immunoassays. Immunofluorescence uses fluorescently-tagged antibodies to detect the presence of their corresponding antigen on a sample. The most commonly used fluorochromes are fluorescein and rhodamine. This technique has its advantages over agglutination because it allows for the detection of just a few cells or viruses, which greatly increases the sensitivity of the test. However, there are additional expenses with this test due to the need for a fluorescence microscope that 
must be well-maintained. Additionally, the person performing the test must have gone through extensive training in order to properly prepare the sample and read the result ${ }^{144}$. The final major type of rapid antigen detection test is the enzyme immunoassay. This is actually a generic term for any test that allows for the detection of antigen-antibody reaction via a resulting enzymatic reaction that produces a colorimetric, fluorimetric or chemiluminescent result. These tests tend to be very customizable so that a wide variety of antigen-antibody complexes can be tested for. The tests have an advantage over others in that a large number of samples can be run in a matter of hours with very little hands-on input from the researcher needed. They also do not usually require the training time that immunoassays do but improper preparation of the sample can result in false positive results. It is also impossible with these tests to check for specimen quality because they do not require the use of a microscope like the previous two described tests do ${ }^{144}$. However, in cellular biology perhaps the most known test for antigen-antibody reactions falls under this category. Enzyme-linked immunosorbent assay (ELISA) is a form of an enzyme immunoassay in which the antibody is linked to a solid phase. A competitive ELISA can be set up with either the antigen or the antibody bound to the solid phase and then a labeled antigen is added along with the patient specimen. These tests are more rapid and specific but less sensitive than the non-competitive ELISA ${ }^{144}$.

Cells can be studied both biochemically and electrokinetically by subjecting the cell population to a treatment and then monitoring the biochemical or dielectrophoretic response to determine if and how the treatment impacted cell composition, morphology, and/or behavior. Infection of erythrocytes with the malaria virus is such a treatment explored from both a biochemical ${ }^{145}$ and electrokinetic ${ }^{4,5}$ perspective. Erythrocytes 
infected with the malaria parasite Plasmodium falciparum express membrane knobs that are antigenically different than the rest of the membrane. Evidence suggests the knob is an electron-dense material below the cell membrane ${ }^{145-148}$, where gold nanoparticles preferentially bind to $P$. falciparum infected erythrocytes ${ }^{145}$. This biochemical evidence of membrane-altercation with malaria infection was reflected in dielectrophoretic characterizations. Gascoyne and colleagues observed changes in the dielectrophoretic spectra and the electrorotation spectra of $P$. falciparum infected erythrocytes and were able to correlate to the biophysical changes such as a leaky membrane observed biochemically.

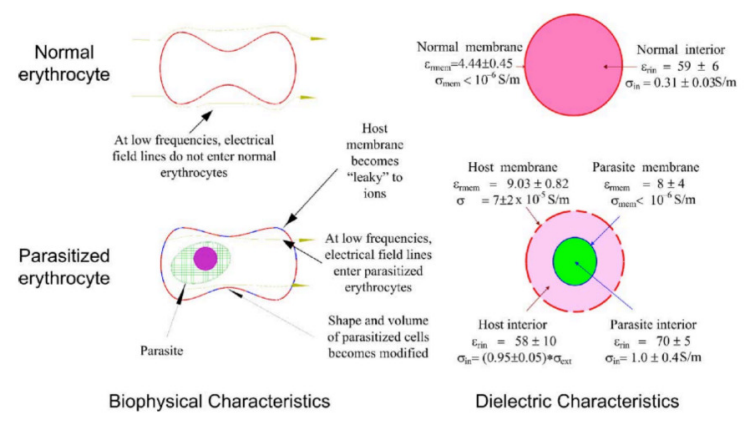

Figure 2.6. Biophysical and dielectric property differences between healthy and parasitized human erythrocytes. Summarized from ${ }^{4}$. Reprinted with kind permission from ${ }^{5 *}$.

At varying medium conductivities, the crossover frequency of the malarial infected erythrocytes is higher than that of the healthy erythrocytes as shown in Figure $2.6^{4}$. This is likely related to the doubling of the membrane permittivity after infection and the order of magnitude increase in membrane conductivity. The cytosolic dielectric properties do not change appreciably outside of the inclusion of the parasite inside the

* Reprinted from Acta Tropica, Vol. 89, Gascoyne P, Satayavivad J, Ruchirawat M. Microfluidic approaches to malaria detection, pp. 357-369, Copyright 2004, with permission from Elsevier. 
erythrocyte $^{4,5}$. The combined biochemical and dielectrophoretic information about malarial infected erythrocytes suggests the infection causes a breakdown in membrane integrity, which leads to greater permittivity of membrane. This allows the electrical field lines to more easily penetrate through the erythrocyte especially at lower frequencies. The increase in membrane conductivity is likely due to the knobs that appear on the membrane since these knobs are made up of electron-dense material that would thereby increase the conductivity of this portion of the cell. Figure 2.6 depicts the permittivity and conductivity changes in the erythrocyte cytosol and membrane as the parasite overtakes the host cell. The parasitic changes to the cell are discernable via dielectrophoretic tools ${ }^{5}$.

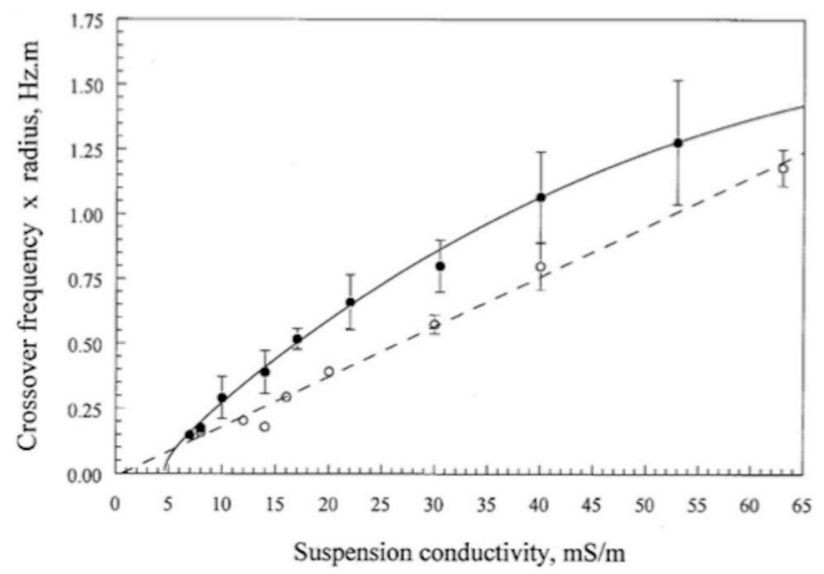

Figure 2.7. Crossover Frequency of healthy human erythrocytes (open symbols) and human erythrocytes infected with Plasmodium falciparum (closed symbols). Reprinted with kind permission from ${ }^{* *}$.

\footnotetext{
** Reprinted from Biochimica et Biophysica Acta (BBA)- Biomembranes, Vol. 1323, Gascoyne P, Pethig R, Satayavivad J, Becker FF, Ruchirawat M. Dielectrophoretic detection of changes in erythrocyte membranes following malarial infection, pp. 240-252, Copyright 1997, with permission from Elsevier.
} 
Cells can also be irreversibly damaged by the dielectrophoretic force, usually in the form of disrupting the integrity of the membrane. Intentional dielectrophoretic cell rupturing has been achieved with much weaker fields than by electroporation ${ }^{149}$. This phenomenon can also serve as an interrogation tool to discern the mechanical properties of the cell membrane.

\subsection{Summary}

The human blood system is comprised of erythrocytes, leukocytes, platelets and the suspending plasma. All three cellular components are derived from the same hematopoietic stem cell but after differentiation have different sizes, shapes and functions. The fields of biochemistry and microbiology have yielded insights into cellular function, genotype/phenotype composition, and morphology. The field of electrokinetics has advanced to determine the dielectric properties of various blood cell types. However, these two research approaches remain disconnected, which hinders the knowledge that can be gained about blood cell function, phenotype, and morphology.

These fields have the opportunity to connect dielectric properties to biochemical or biophysical properties of the cell. A few notable groups have begun to accomplish this including extensive work on exploring difference in leukocyte subpopulations ${ }^{12-14,150}$, differentiating healthy and $P$. falciparum infected erythrocytes ${ }^{4,5}$ and exploring the molecular-level changes between the same erythrocyte types ${ }^{1,10,11,145-148,151-156}$. Much progress remains to be done to closely couple the biochemical phenotype to the dielectric properties.

Combining well-established microbiology techniques for cellular analysis with electrokinetic interrogations could yield more, higher quality information. Further, 
coordination between these fields could increase the rate of knowledge acquired in the fields. As relationships between observed dielectric properties are coupled to the corresponding biophysical change the cell, this knowledge could lead to a priori knowledge or predictions of the nature of biophysical changes in cell populations. Electrokinetic tests have advantages such as intact cell interrogation, rapid measurements, portability, low cost, no chemical additives and small volume usage. The coupling of microbiological information with dielectric information has the potential to push the field of cellular analysis forward rapidly. 


\section{ELECTROKINETICS AND DIELECTROPHORESIS: THEORY AND USE IN MICRODEVICES}

\subsection{Introduction}

The force isolated in these experiments is alternating current dielectrophoresis, which belongs to a much larger group of forces known as electrokinetic phenomena. Electrokinetic phenomena are a group of effects that happen when electric fields are applied to conductive fluids including, but not limited to the fields of electrokinetics: electrophoresis, electro-osmosis, diffusiphoresis, capillary osmosis, sedimentation potential, streaming potential/current, colloid vibration current and electric sonic amplitude. In classic electrokinetic theory, as a particle surface acquires a net charge, a polarization occurs on the surface and hence around the particle. This polarization causes ions of the opposite charge (counter-ions) to concentrate adjacent to the surface to form what is known as the Debye double layer. Four of the above discussed electrokinetic phenomena exhibit this behavior: electro-osmosis, electrophoresis, streaming potential and sedimentation potential ${ }^{12}$. This section will focus on AC dielectrophoresis (AC DEP) and its theory and use in microdevices.

Dielectrophoresis was researched by H.A. Pohl for almost 20 years prior to publishing his milestone book in 1978. He defined dielectrophoresis as the movement of polarizable particles in a nonuniform electric field ${ }^{15}$; it is now wildly explored as a way to quickly separate, manipulate, and identify bioparticles ${ }^{1,3-7,12-14,17,23,116,118,123,124,140,157-173}$ on a micrometer or nanometer scale ${ }^{129}$. Dielectrophoretic studies on erythrocytes include removal of leukemia cells from blood ${ }^{3}$ and separation of cells affected by malaria from 
unharmed cells ${ }^{5}$. The dielectrophoretic force can also irreversibly damage cells, either accidentally or as an intentional mechanism for cell rupture as discussed in Chapter 4. Cell rupture by dielectrophoresis uses much weaker fields than by electroporation and is likely due to a disruption of lipid/lipid interactions in the membrane surrounding the cell $^{149}$. Discussed herein is the physics behind dielectrophoretic theory, the various models most commonly used to predict particle behavior as a result of the dielectrophoretic force and the use of dielectrophoresis in microdevice technology both with regards to particles and biological cells.

There are general dielectrophoretic behavior trends for most particles, especially cells, when subjected to alternating current as shown in Figure 3.1. Typically for cells the low frequency region between $1-100 \mathrm{kHz}$ is characterized by negative DEP behavior. Somewhere after $100 \mathrm{kHz}$ there is a transition to positive DEP behavior, which tends to last until around $100 \mathrm{MHz}^{164}$. With cells, this second transition (COF) back to nDEP occurs because the cell has a membrane and therefore the current must pass through two different dielectrics. Cells also generally start with nDEP behavior because the cell is less polarizable than the medium in this region and the current would rather pass through the medium than the cell, so the cells are pushed to areas of low field strength. 


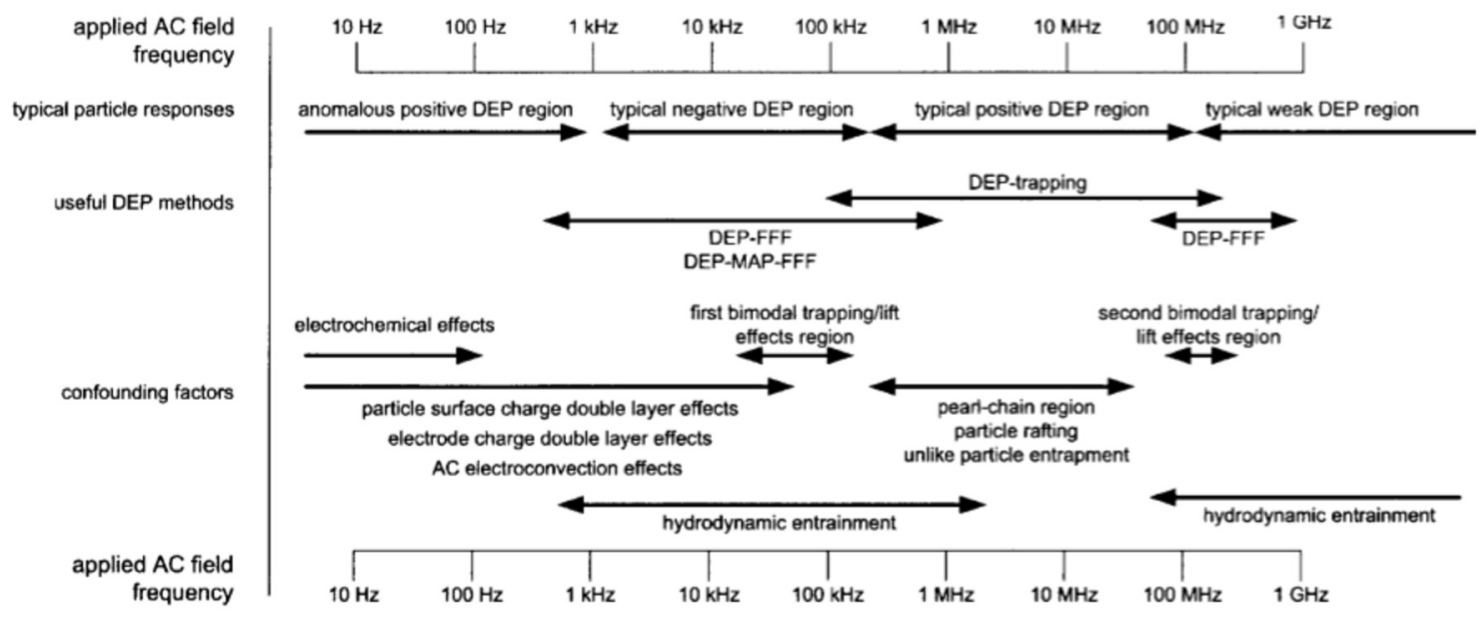

Figure 3.1. Guide to frequency regions at which specific DEP behaviors typically occur, as well as information about what methodology is appropriate in these regions. Reprinted with permission from ${ }^{164}$.

\subsection{Dielectrophoresis}

Alternating current dielectrophoresis is defined as the movement of particles in an inhomogeneous field. Based on the particle's intrinsic polarizability as compared to that of the suspending medium, it will experience either positive dielectrophoresis and move towards a region of high field density or negative dielectrophoresis and move towards a region of low field density ${ }^{15}$. As will be seen later the polarizability of the particle is determined by its perimittivity and conductivity, as well as the frequency of the field being applied. Therefore, the intrinsic polarizability of the particle can change as both a function of its components and as a function of the frequency of the applied field. In order to derive the governing equations for this theory we need to start with a dipole and sum of the forces acting upon the dipole. 


\subsubsection{Spherical Model}

To begin, consider a dipole in a molecule crudely represented by $+\mathrm{q}$ on one side and $-\mathrm{q}$ on the opposing side as Figure 3.2.

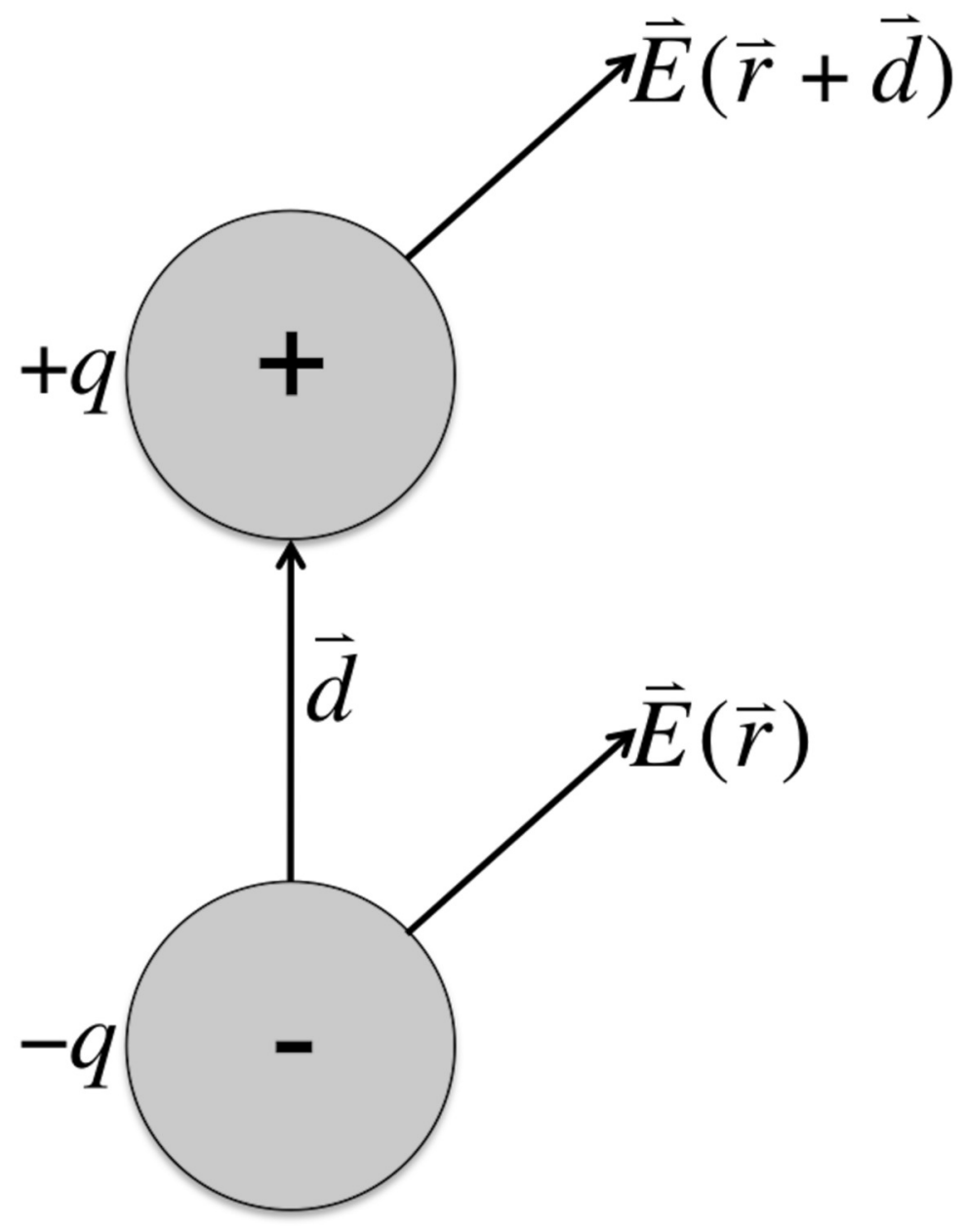

Figure 3.2. Basic diagram showing the electric field on each dipole side. The force on the dipole is the electric fields are multiplied by the charge on the dipole half and summed.

Summing the forces on the dipole shown in Figure 3.2 give:

$$
\vec{F}=q \vec{E}(\vec{r}+\vec{d})=q \vec{E}(\vec{r})
$$

and when $\mathrm{d}$ is small compared to the electric field, we can use a Taylor series expansion to simplify this expression for the electric force

$$
\vec{E}(\vec{r}+\vec{d})=\vec{E}(\vec{r})+\vec{d} \bullet \nabla \vec{E}(\vec{r})+\ldots
$$


where all higher order terms can be neglected. Substituting the first two terms of (2) into (1) gives:

$$
\vec{F}=q \vec{d} \bullet \nabla \vec{E}(\vec{r})
$$

where the dipole moment is defined as $\vec{p} \equiv q \vec{d}$. This gives us a final equation for the dipole force:

$$
\vec{F}_{\text {dipole }}=\vec{p} \bullet \nabla \vec{E}
$$

Therefore, in order to have a net force on a dipole, the external electric field must be spatially nonuniform because otherwise the gradient of the field would be zero ${ }^{114}$. Pohl identified dielectrophoresis with the real part of the second term in Equation (4), the movement of a particle in a nonuniform field ${ }^{15}$. Since Pohl, dielectrophoresis has been expanded to include particle quadrupoles (Q) and higher order terms ${ }^{114}$.

Poisson's equation must hold true for the electric fields as well, which relates them to the electrostatic potential

$$
\nabla \bullet(\varepsilon \vec{E})=-\nabla \bullet(\varepsilon \nabla \Phi) \quad(5)^{174}
$$

Now must solve for a general expression for the electrostatic potential:

$$
\Phi(r, \theta)=\frac{q}{4 \pi \varepsilon_{m} r_{+}}-\frac{q}{4 \pi \varepsilon_{m} r_{-}}
$$

We can define $r_{+}$and $r$. by means of $d, r$ and $\theta$

$$
\begin{aligned}
& \left(\frac{r}{r_{+}}\right)=\left[1+\left(\frac{d}{2 r}\right)^{2}-\frac{d}{r} \cos \theta\right]^{-1 / 2} \\
& \left(\frac{r}{r_{-}}\right)=\left[1+\left(\frac{d}{2 r}\right)^{2}+\frac{d}{r} \cos \theta\right]^{-1 / 2}
\end{aligned}
$$


Equations (7) and (8) can be expanded using a Maclaurin series to get

$$
\begin{aligned}
& \left(\frac{r}{r_{+}}\right)=P_{0}+\left(\frac{d}{2 r}\right) P_{1}+\left(\frac{d}{2 r}\right)^{2} P_{2}+\left(\frac{d}{2 r}\right)^{3} P_{3}+\ldots \\
& \left(\frac{r}{r_{-}}\right)=P_{0}-\left(\frac{d}{2 r}\right) P_{1}+\left(\frac{d}{2 r}\right)^{2} P_{2}-\left(\frac{d}{2 r}\right)^{3} P_{3}+\ldots
\end{aligned}
$$

where $\mathrm{P}_{0}, \mathrm{P}_{1}, \mathrm{P}_{2}$, and $\mathrm{P}_{3}$, are the Legendre polynomials. We can solve for an expression for the electrostatic potential in terms of $r$ and $\theta$ by combining Equations (9) and (10) with Equation (6)

$$
\Phi(r, \theta)=\frac{q d P_{1} \cos \theta}{4 \pi \varepsilon_{m} r^{2}}+\frac{q d^{3} P_{3} \cos \theta}{4 \pi \varepsilon_{m} r^{4}}+\ldots
$$

The first term of Equation (11) is the dipole electrostatic potential and the second term is the octupolar correction term.

Now that we have a general expression for the electrostatic potential, we can look at the specific case of a lossless dielectric sphere in a dielectric medium and subjected to a z-direction electric field of magnitude $\mathrm{E}_{0}$ as seen in Figure 3.3. In order to solve this problem we need to make the following assumptions:

1. Electric field, $\mathrm{E}_{0}$, is uniform close to the particle

2. No free charge in sphere

3. No free charge in dielectric medium around the sphere

4. Presence of sphere does not significantly disturb the system that creates $\mathrm{E}_{0}$ 


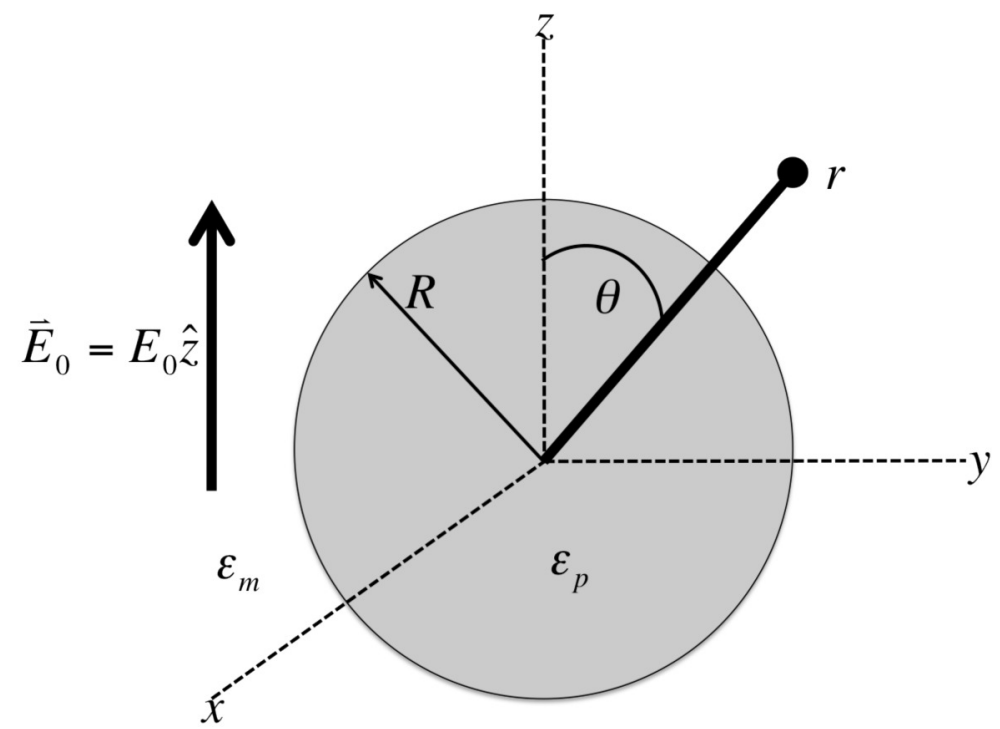

Figure 3.3. Representation of a dielectric sphere in a dielectric medium that is used to solve for the electrostatic potential.

The solution to the electrostatic potential must satisfy Laplace's equation because the electrostatic field must be both divergence and curl free to meet \#1 and \#4 above

$$
\nabla^{2} \Phi=0
$$

Two solutions to Equation (12) have previously been obtained for the outside $\Phi_{1}$ and inside $\Phi_{2}$ electrostatic potential ${ }^{114}$

$$
\begin{gathered}
\Phi_{1}(r, \theta)=-E_{0} r \cos \theta+\frac{A \cos \theta}{r^{2}}, \quad r>R \\
\Phi_{2}(r, \theta)=-B r \cos \theta, \quad r>R
\end{gathered}
$$

In Equation (13) the first term is the imposed uniform electrostatic field and the second term is the induced dipole term resulting from the particle interacting with the field. In order to solve for the constants A and B, we must impose two boundary conditions at the sphere boundary $(\mathrm{r}=\mathrm{R})$. The first boundary condition is the continuity condition: the electrostatic potential must be continuous across the particle-fluid boundary

$$
\Phi_{1}(R, \theta)=\Phi_{2}(R, \theta) \quad(\text { B.C. } 1)
$$


The second boundary condition states that the normal component of the displacement flux vector which is the must also be continuous across the particle-fluid boundary with finite permittivities $\varepsilon_{\mathrm{m}}$ (medium) and $\varepsilon_{\mathrm{p}}$ (particle)

$$
\left.\varepsilon_{m} \frac{\partial \Phi_{1}}{\partial r}\right|_{r=R}=\left.\varepsilon_{p} \frac{\partial \Phi_{2}}{\partial r}\right|_{r=R}
$$

In order to satisfy boundary condition 2 we must take the derivative with respect to position of Equations (13) and (14) to obtain the flux vectors.

$$
\begin{gathered}
\frac{\partial \Phi_{1}}{\partial r}=-E_{0} \cos \theta-\frac{2 A \cos \theta}{r^{3}} \\
\frac{\partial \Phi_{2}}{\partial r}=-B \cos \theta
\end{gathered}
$$

Using B.C.1 and B.C.2 in conjunction with Equations 13-16, we can solve for the constants A and B

$$
\begin{array}{r}
A=R^{3} E_{0}\left(\frac{\varepsilon_{p}-\varepsilon_{m}}{\varepsilon_{p}+2 \varepsilon_{m}}\right) \\
B=E_{0}\left(\frac{3 \varepsilon_{m}}{\varepsilon_{p}+2 \varepsilon_{m}}\right)
\end{array}
$$

When the dipole term in Equation (11) is compared to the induced dipole term in Equation (13) the effective moment becomes

$$
p_{\text {eff }}=4 \pi \varepsilon_{m} A=4 \pi \varepsilon_{m} f_{C M} R^{3} E_{0}
$$

where $\mathrm{f}_{\mathrm{CM}}$ is the Clausius-Mossotti factor, which is given by

$$
f_{C M}=\frac{\varepsilon_{p}-\varepsilon_{m}}{\varepsilon_{p}+2 \varepsilon_{m}}
$$


All of the above equations were valid for a homogenous dielectric sphere with no ohmic $\operatorname{loss}^{114}$.

The dipole moment therefore exhibits a time delay when the field is applied suddenly or a phase lag when the applied field is sinusoidal. In addition to having finite permittivities ( $\varepsilon_{\mathrm{m}}$ and $\varepsilon_{\mathrm{p}}$ ) as they do in the lossless case, the medium and sphere now also have finite conductivities $\left(\sigma_{\mathrm{m}}\right.$ and $\left.\sigma_{\mathrm{p}}\right)$. In an alternating current electric field, the applied field will have the form:

$$
\vec{E}(t)=\operatorname{Re}\left[E_{0} \hat{z} \exp (i \omega t)\right]
$$

Solutions in form of Equations (13) and (14) still hold as long as the assumption can now be made that the constants $\mathrm{A}$ and $\mathrm{B}$ are complex. The continuity electrostatic boundary condition (B.C.1) remains unchanged but the displacement flux vector (B.C.2) must be replaced by a charge continuity condition because the finite conductivity allows for a time-dependent accumulation of free electrical surface charge

$$
J_{r_{1}}-J_{r_{2}}+\frac{\partial \sigma_{f}}{\partial t}=\left.0\right|_{r=R}
$$

where $\mathrm{J}_{\mathrm{r} 1}$ and $\mathrm{J}_{\mathrm{r} 2}$ are the normal components of the ohmic current outside and inside the dielectric sphere, respectively and $\sigma_{\mathrm{f}}$ is the free electric surface charge

$$
\begin{gathered}
J_{r_{1}}=\sigma_{m} E_{r_{1}} \\
J_{r_{2}}=\sigma_{p} E_{r_{2}} \\
\sigma_{f}=\varepsilon_{m} E_{r_{1}}-\varepsilon_{p} E_{r_{2}}
\end{gathered}
$$

We can now assume a periodic time dependence and for convenience use Euler's exponential form $\exp (i \omega t)$ for all variables and can substitute $i \omega$ for $\frac{\partial}{\partial t}$ to transform into 
the frequency domain. When combining this with equations $21-23$, boundary condition 3 becomes

$$
\varepsilon_{m}^{*} E_{r_{1}}^{*}(r=R, \theta)=\varepsilon_{p}^{*} E_{r_{2}}^{*}(r=R, \theta)
$$

where * indicates a complex term and the complex permittivities are given by

$$
\begin{gathered}
\varepsilon_{m}^{*}=\varepsilon_{m}+\frac{\sigma_{m}}{i \omega} \\
\varepsilon_{p}^{*}=\varepsilon_{p}+\frac{\sigma_{p}}{i \omega}(26)
\end{gathered}
$$

Using these new boundary conditions we can then solve for $\mathrm{A}^{*}$ and $\mathrm{B}^{*}$ in equations (15) and (16). They will keep the same form as before except the complex permittivity will replace the real permittivity.

$$
\begin{gathered}
A=R^{3} E_{0}\left(\frac{\varepsilon_{p}^{*}-\varepsilon_{m}^{*}}{\varepsilon_{p}^{*}+2 \varepsilon_{m}^{*}}\right) \\
B=E_{0}\left(\frac{3 \varepsilon_{m}^{*}}{\varepsilon_{p}^{*}+2 \varepsilon_{m}^{*}}\right)
\end{gathered}
$$

Equation (19) now becomes the complex effective moment

$$
p_{e f f}=4 \pi \varepsilon_{m} A^{*}=4 \pi \varepsilon_{m} f_{C M}^{*} R^{3} \vec{E}_{0}
$$

where $\mathrm{f}_{\mathrm{CM}}$ is the complex Clausius-Mossotti factor, which is given by

$$
f_{C M}^{*}=\frac{\varepsilon_{p}^{*}-\varepsilon_{m}^{*}}{\varepsilon_{p}^{*}+2 \varepsilon_{m}^{*}}
$$

Now that we have an expression for the effective moment for both a lossless (Equation 19) and lossy sphere (Equation 29), we can obtain an expression for the dielectrophoretic force for both cases ${ }^{114}$. 
In order to determine the dielectrophoretic force for a lossless sphere, we must substitute Equation (19), the effective moment, into the general dielectrophoretic force Equation (4) as follows

$$
\vec{F}_{D E P}=4 \pi \varepsilon_{m} f_{C M} R^{3} \vec{E}_{0} \bullet \nabla \vec{E}_{0}
$$

The following vector calculus identity helps translate Equation (31) into a more recognizable form

$$
\frac{1}{2} \nabla\left(\vec{E}_{0} \bullet \vec{E}_{0}\right)=\vec{E}_{0} \times\left(\nabla \times \vec{E}_{0}\right)+\left(\vec{E}_{0} \bullet \nabla\right) \vec{E}_{0}
$$

This identity is useful because the second term is the curl of the electric field, which we have set to be zero in our assumptions. Therefore Equation (32) becomes

$$
\frac{1}{2} \nabla\left(\vec{E}_{0}^{2}\right)=\left(\vec{E}_{0} \bullet \nabla\right) \vec{E}_{0}
$$

Substituting Equation (32) into Equation (30) gives the lossless dielectrophoretic force

$$
\vec{F}_{D E P}=2 \pi \varepsilon_{m} f_{C M} R^{3} \nabla \vec{E}_{0}^{2}
$$

which is the most common form of the dielectrophoretic force equation; it was first established by Pohl in $1951^{15,114}$.

For particles with dielectric loss, Equation (31) becomes

$$
\vec{F}_{D E P}(t)=\operatorname{Re}\left[\vec{p}_{\text {eff }}^{*} \exp (i \omega t)\right]=\operatorname{Re}\left[\vec{E}^{*} \exp (i \omega t)\right]
$$

The time-average of Equation (35) yields

$$
\rangle \vec{F}_{D E P}\left\langle=2 \pi \varepsilon_{m} R^{3}\left[f_{C M}(\omega)\right] \nabla \vec{E}_{r m s}^{2}\right.
$$

We therefore have derived the dielectrophoretic force equation and the Clausius-Mossotti factor for both a lossless and a lossy dielectric sphere. These equations are often sufficient for explaining the behavior of particles in a dielectrophoretic field, however a 
more involved model is needed for most biological particles because the particle core has different dielectric properties than the particle shell.

\subsubsection{Core-Shell Model}

The expressions thus far represent either a homogenous sphere without or with ohmic loss manifesting with the absence or presence of particle/media conductivity. However, most biological particles are heterogeneous, having at the very least an outer membrane surrounding cellular cytoplasm. For this section we consider the same type of lossy dielectric sphere as above with a radius $r_{p}$ and a core with a radius $r_{c}$. The electrostatic field follows Laplace's Equation (12) with the following boundary conditions

$$
\begin{gathered}
\left.\varepsilon_{m} \frac{\partial \Phi}{\partial r}\right|_{r=r_{p}}=\left.\varepsilon_{s} \frac{\partial \Phi}{\partial r}\right|_{r=r_{p}} \\
\left.\varepsilon_{s} \frac{\partial \Phi}{\partial r}\right|_{r=r_{c}}=\left.\varepsilon_{c} \frac{\partial \Phi}{\partial r}\right|_{r=r_{c}} \\
\Phi=-\left.E_{0} z\right|_{r \rightarrow \infty}
\end{gathered}
$$

This system can be solved similar to the homogeneous sphere system of equations. The solution to the effective moment is

$$
p_{\text {eff }}=4 \pi \varepsilon_{m} f_{C M}^{*} r_{p}^{3} \vec{E}_{0}
$$

The Clausius-Mossotti factor in this case is given by:

$$
f_{C M}^{*}=\frac{\varepsilon_{p}^{*}-\varepsilon_{m}^{*}}{\varepsilon_{p}^{*}+2 \varepsilon_{m}^{*}}
$$

where the complex permittivity of the particle is given by: 


$$
\varepsilon_{p}^{*}=\varepsilon_{s}^{*}\left[\frac{\frac{r_{p}^{3}}{r_{c}^{3}}+\frac{\varepsilon_{c}^{*}-\varepsilon_{s}^{*}}{\varepsilon_{c}^{*}+2 \varepsilon_{s}^{*}}}{\frac{r_{p}^{3}}{r_{c}^{3}}-\frac{\varepsilon_{c}^{*}-\varepsilon_{s}^{*}}{\varepsilon_{c}^{*}+2 \varepsilon_{s}^{*}}}\right]
$$

Parameters with a subscript "s" represent shell parameters and subscript "c" is included on core parameters. The whole radius of the particle is $r_{p}$ and the radius of just the core is $r_{c}$. This idea of a core-shell model can be extended to multiple shells, always working from the inside towards the outside ${ }^{114,115}$. As will be seen in later chapters, there are differences in the DEP behavior of human erythrocytes based on their ABO-Rh antigen expression. It is possible that a way to correlate the behavior of these erythrocytes with the theory by adding an extra shell to the theoretical model that will take into account the presence or absence of the $\mathrm{A}, \mathrm{B}$ and $\mathrm{O}$ antigens.

The complex permittivity of the particle can take on a simplified form when the shell is extremely thin when compared to the core. A linear expansion of the first term in Equation (39) is permissible because the shell is extremely thin compared to the core and the result is as follows:

$$
\frac{r_{p}^{3}}{r_{c}^{3}}=1+3 \frac{\Delta r}{r_{p}}
$$

where $\Delta r=r_{p}-r_{c}$ and $\Delta r<<r_{p}$. When Equation (40) is inserted into Equation (39) the complex permittivity of a particle with a thin shell is simplified to

$$
\varepsilon_{p}^{*}=\varepsilon_{s}^{*}\left[\frac{\varepsilon_{c}^{*}+\frac{\Delta r}{r_{p}}\left(\varepsilon_{c}^{*}+2 \varepsilon_{s}^{*}\right)}{\varepsilon_{s}^{*}+\frac{\Delta r}{r_{p}}\left(\varepsilon_{c}^{*}+2 \varepsilon_{s}^{*}\right)}\right]
$$


When the conductivity of the core is much greater than the shell and the core permittivity is also much greater than the shell, assumptions that hold true for human erythrocytes, than further simplification of Equation (41) is possible because the following is true

$$
\varepsilon_{c}^{*}+2 \varepsilon_{s}^{*} \approx \varepsilon_{c}^{*}(42)
$$

Equation (41) therefore becomes

$$
\varepsilon_{p}^{*}=\varepsilon_{s}^{*}\left[\frac{\varepsilon_{c}^{*}+\frac{\Delta r}{r_{p}} \varepsilon_{c}^{*}}{\varepsilon_{s}^{*}+\frac{\Delta r}{r_{p}} \varepsilon_{c}^{*}}\right]=\left[\frac{\varepsilon_{c}^{*} \varepsilon_{s}^{*}}{\frac{\Delta r}{r_{p}} \varepsilon_{c}^{*}+\varepsilon_{s}^{*}}\right] \quad(43)^{115,117 .}
$$

This model, with the thin-shell assumption, works well for most biological cells because the cell membrane is extremely thin compared to the core and the core of the cell is always more conductive and permeable than the membrane surrounding it ${ }^{115,117}$. However, the particle shape in the above models is assumed to be spherical. A lot of cells, erythrocytes in particular, are not actually spherical in shape. Ellipsoidal models are a better fit geometrically for the biconcave shape of the human erythrocyte.

\subsubsection{Ellipsoid Model}

To solve for a particle's effective moment, the field applied is taken into account as well as the field created by the particle. The particle-created field is highly dependent on particle shape, so deviation from a spherical shape must be included in the dipole moment. Ellipsoidal particles have three axes: $\mathrm{r}_{1}$ (major) and $\mathrm{r}_{2}$ and $\mathrm{r}_{3}$ (minor) ${ }^{114,115,117}$. For an oblate ellipsoid, $r_{1}$ is equal to $r_{2}$ and these are both larger than $r_{3}$. Human erythrocytes are geometrically classified as oblate ellipsoids. Each axes therefore has its own dipole moment given by 


$$
p_{0, i}^{*}=4 \pi r_{1} r_{2} r_{3} \varepsilon_{m} f_{C M, i}^{*} E_{0, i}
$$

The Clausius-Mossotti factor for each axis, $\mathrm{i}$, is

$$
f_{C M, i}^{*}=\frac{\varepsilon_{p}^{*}-\varepsilon_{m}^{*}}{3\left[\varepsilon_{m}^{*}+\left(\varepsilon_{p}^{*}-\varepsilon_{m}^{*}\right) L_{i}\right]}
$$

where $L_{i}$ is the depolarization factor for each axis, $\mathrm{i}$, that takes into account the eccentricity, the amount by which it deviates from spherical geometry, of the particle via the following axis relationship

$$
L_{i}=\frac{r_{1} r_{2} r_{3}}{2} \int_{0}^{\infty} \frac{1}{\left(l+r_{i}^{2}\right) \sqrt{\left(l+r_{1}^{2}\right)\left(l+r_{2}^{2}\right)\left(l+r_{3}^{2}\right)}} d l
$$

with a dummy variable of integration $l$. If we take into account the fact that $\mathrm{a}_{1}$ is equal to $\mathrm{a}_{2}$ for the oblate ellipsoid geometry that models a human erythrocyte most accurately, we can substitute $\mathrm{a}_{1}$ in for $\mathrm{a}_{2}$ and Equation (46) becomes

$$
L_{i}=\frac{r_{1}^{2} r_{3}}{2} \int_{0}^{\infty} \frac{1}{\left(l+r_{i}^{2}\right)\left(l+r_{1}^{2}\right) \sqrt{\left(l+r_{3}^{2}\right)}} d l
$$

For the major axes the depolarization factor is

$$
L_{1}=\frac{r_{1}^{2} r_{3}}{2} \int_{0}^{\infty} \frac{1}{\left(l+r_{1}^{2}\right)^{2} \sqrt{\left(l+r_{3}^{2}\right)}} d l
$$

and the for the minor axis is

$$
L_{3}=\frac{r_{1}^{2} r_{3}}{2} \int_{0}^{\infty} \frac{1}{\left(l+r_{3}^{2}\right)^{3 / 2}\left(l+r_{1}^{2}\right)} d l
$$

These depolarization factors can be analytically integrated to determine a non-integral expression for $L_{1}$ and $L_{3}$ that can be used in Equation (45) to determine the ClausiusMossotti factor for both the major and minor axes ${ }^{114,115,117}$. If using the ellipsoidal model 
it is best to use a program such as Matlab or Mathematica to calculate the depolarization factors and from there move on to the Clausius-Mossotti factor and DEP force because the integrals of the depolarization factors are indefinite and the researcher must take the limit of them as they go to infinity. For the major axis this will result in an infinite number so then L'Hopital's rule must be used to further simplify the answer to that integral.

\subsubsection{Review of Models}

The main models for dielectrophoretic behavior of homogeneous and nonhomogeneous spheres and ellipsoids have been discussed and are summarized in Table 3.1. References use the specified theoretical model to explain the behavior of the particles/cells in their system. 


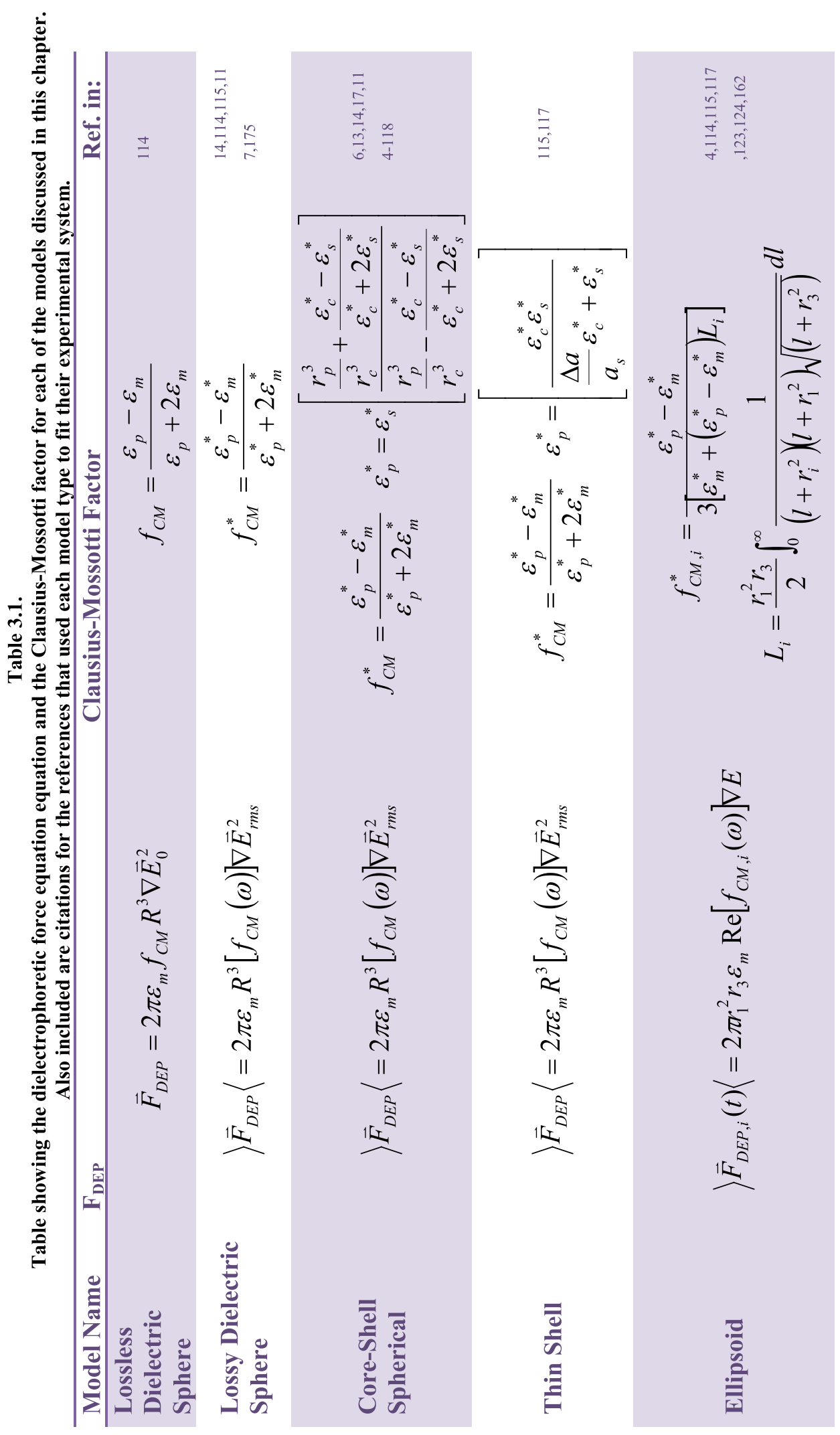


Using previously published values for the membrane and cytoplasm conductivity and permittivity ${ }^{3-5,123,176-179}$, various models discussed above for human erythrocytes were plotted. The Matlab code used to make these models can be found in Appendix 9.3. The human erythrocyte was assumed to be $3.5^{*} 10^{-6} \mathrm{~m}$ by $1 * 10^{-6} \mathrm{~m}$ in diameter with a membrane thickness of $4.6^{*} 10^{-9} \mathrm{~m}^{26}$. Figure 3.4A shows the basic spherical model using $0.31 \mathrm{~S} / \mathrm{m}$ as the cytoplasm conductivity and 59 for the cytoplasm permittivity ${ }^{5}$. This basic model allowed observation of the high frequency transition from $\mathrm{pDEP}$ to $\mathrm{nDEP}$ behavior at $35 \mathrm{MHz}(0.01 \mathrm{~S} / \mathrm{m})$ and $37 \mathrm{MHz}(0.1 \mathrm{~S} / \mathrm{m})$ but completely misses the low frequency transition from nDEP to pDEP. The Core-Shell model in Figure 3.4B demonstrates the low frequency transition because the model accounts for the current passing through two different dielectrics (cytosol and membrane) and thus gives the two points of cross-over behavior. The parameters used for the Core-Shell and Ellipsoidal models were $10^{-7} \mathrm{~S} / \mathrm{m}$ membrane conductivity, 4.44 membrane permittivity, $0.31 \mathrm{~S} / \mathrm{m}$ cytoplasm conductivity and 59 cytoplasm permittivity ${ }^{5}$. Due to the polarization factors in Equations (48) and (49), the minor axis (Figure 3.4C) and major axis (Figure 3.4D) are shown separately for the ellipsoidal model. For the experiments presented in this dissertation, the cells were always found to be laying parallel to the plated electrodes, so the experienced field followed along the major axis and this was the axis used in ellipsoid model predictions for behavior. If a 3D field was used and observed then a combination of the two axes models would need to be used. The medium parameters were a permittivity of 136 , assumed to be similar to $\mathrm{PBS}^{179}$, and conductivities of $0.01 \mathrm{~S} / \mathrm{m}$ (red), $0.1 \mathrm{~S} / \mathrm{m}$ (green) and $0.9 \mathrm{~S} / \mathrm{m}$ (blue). The real part of the Clausius-Mossotti factor, which determines if a particle is experiencing pDEP or nDEP, was calculated according to Equations (30), (41), 
(48) and (49) for frequencies between $10 \mathrm{kHz}\left(10^{4} \mathrm{~Hz}\right)$ and $100 \mathrm{MHz}\left(10^{8} \mathrm{~Hz}\right)$. The calculated theoretical COF values for the four models are shown in Table 3.2. There are no values shown for any of the models at $0.9 \mathrm{~S} / \mathrm{m}$ because theoretically the cells should experience only $\mathrm{nDEP}$ behavior across the entire $10 \mathrm{kHz}$ to $100 \mathrm{MHz}$ range at this medium conductivity. In Chapter 5 this theoretical behavior is shown to not match with the experimental results ${ }^{1}$.
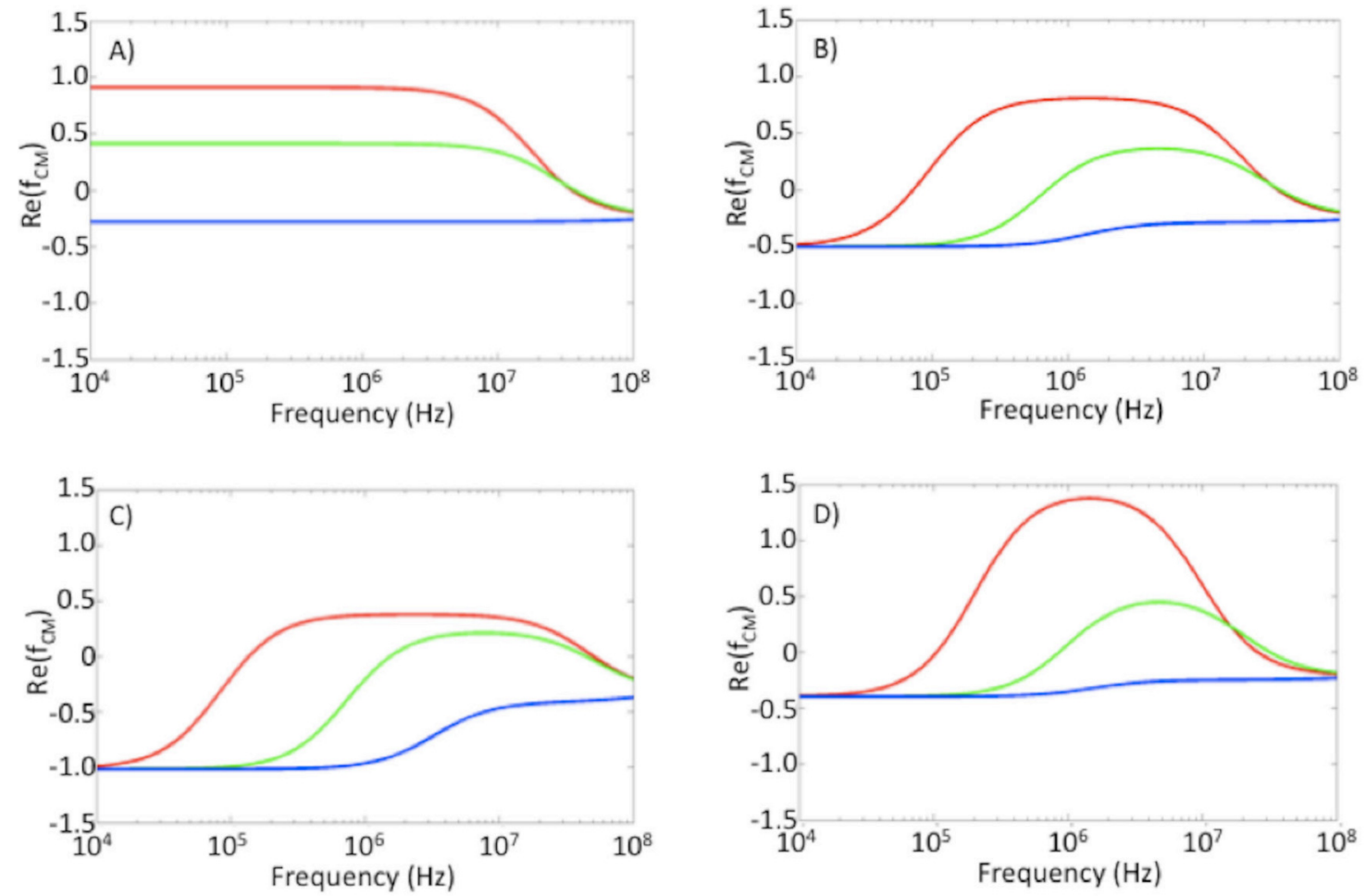

Figure 3.4. Theoretical plots corresponding to the four models discussed above Spherical (A), Shelled Spherical (B), Ellispoidal-Minor (C) and Elllipsoidal-Major (D). Shown are the three medium conductivities tested: $0.01 \mathrm{~S} / \mathrm{m}$ (red), $0.1 \mathrm{~S} / \mathrm{m}$ (green) and $0.9 \mathrm{~S} / \mathrm{m}$ (blue). Both the Shelled Spherical and the Ellipsoidal models show the two-COF behavior that is typical of cells due to their double dielectric nature. 
Table 3.2.

Shows COF values (in MHz) for the nDEP to pDEP transition and the pDEP to nDEP transition for the four models graphed above in Figure 3.4. Values were calculated by fitting a linear equation between the two points closest to zero as calculated by Matlab.

\begin{tabular}{|c|c|c|c|c|c|c|c|c|}
\hline Freq & \multicolumn{2}{|c|}{ Spherical } & \multicolumn{2}{c|}{ Core-Shell } & \multicolumn{2}{c|}{ Ellipsoid Minor } & \multicolumn{2}{c|}{ Ellipsoid Major } \\
\hline (MHZ) & $0.01 S / m$ & $0.1 S / m$ & $0.01 S / m$ & $0.1 S / m$ & $0.01 S / m$ & $0.1 S / m$ & $0.01 S / m$ & $0.1 S / m$ \\
\hline n to p & & & 0.07 & 0.70 & 0.13 & 1.56 & 0.11 & 0.87 \\
\hline p to n & 35.44 & 36.86 & 35.28 & 36.52 & 50.41 & 44.26 & 25.61 & 31.41 \\
\hline
\end{tabular}

Because the ellipsoidal model has two axes, it is important to study how these axes differ from one another at all three conductivities of interest. Figure 3.5 demonstrates that the frequency range of $\mathrm{pDEP}$ behavior, indicated by positive $\operatorname{Re}\left(\mathrm{f}_{\mathrm{CM}}\right)$ values, decreased by $5 \mathrm{MHz}$ for the major axis and increased by $7 \mathrm{MHz}$ for the minor axis as medium conductivity increases from $0.01 \mathrm{~S} / \mathrm{m}(\mathrm{A})$ to $0.1 \mathrm{~S} / \mathrm{m}(\mathrm{B})$. For the medium conductivity of $0.9 \mathrm{~S} / \mathrm{m}(\mathrm{C})$ all behavior was $\mathrm{pDEP}$. For all graphs, the major axis is the solid line and the minor axis is dashed. The minor axis, which refers to the height of the erythrocyte, experiences the cross-over frequency (COF) at a higher frequency than the major axis in mediums of both $0.01 \mathrm{~S} / \mathrm{m}$ and $0.1 \mathrm{~S} / \mathrm{m}$. For the highest medium conductivity, COFs are not observed; however, the minor axis consistently experiences stronger $\mathrm{nDEP}$ than the major axis. The major axis consistently experiences stronger pDEP behavior at $0.01 \mathrm{~S} / \mathrm{m}$ and $0.1 \mathrm{~S} / \mathrm{m}$ than the minor axis. This is likely because the cell membrane to axis radius ratio, which shows up in Equations (39) and (40) as $\frac{\Delta a}{a_{s}}$, is 3.5 times larger for the minor axis than for the major axis. Thus, the cytoplasm contribution is much smaller for the major axis so membrane characteristics tend to dominate behavior leading to more polarizable particles than the medium at the lower frequencies. At higher frequencies, where permittivity dominates behavior, the less 
permeable membrane promotes $\mathrm{nDEP}$ cell behaviors at a lower frequency for the major axis than for the minor. In the experiments described in this work, the cells orient parallel to the electrodes, so the majority of the electric field aligns along the cell's major axis. Therefore, the behavior of the erythrocytes described herein more closely follows the major axis theoretical behavior.

These theoretical graphs show overall, ideal behavior and thus do not match experimental results in Chapters 5 and 6 perfectly. Theory assumes all erythrocytes are perfectly symmetric, have uniform properties, and are all the same. Experimentally, this is not the case; there are definite differences in the frequency at which cells within a population begin to experience $\mathrm{pDEP}$ and the range over which they experience each behavior ${ }^{1}$. Additional parameters should be added to the current models to account for polarizability differences between ABO-Rh blood types. As discussed above, the membrane dielectric constant heavily influences DEP behavior, especially as the shape approaches the ellipsoidal case. The dielectric constant is thus affected by changes in surface and transmembrane antigen expression. Evidence for cell type yielding appreciable differences in DEP behavior is presented in Chapter 3 in extensive detail. 

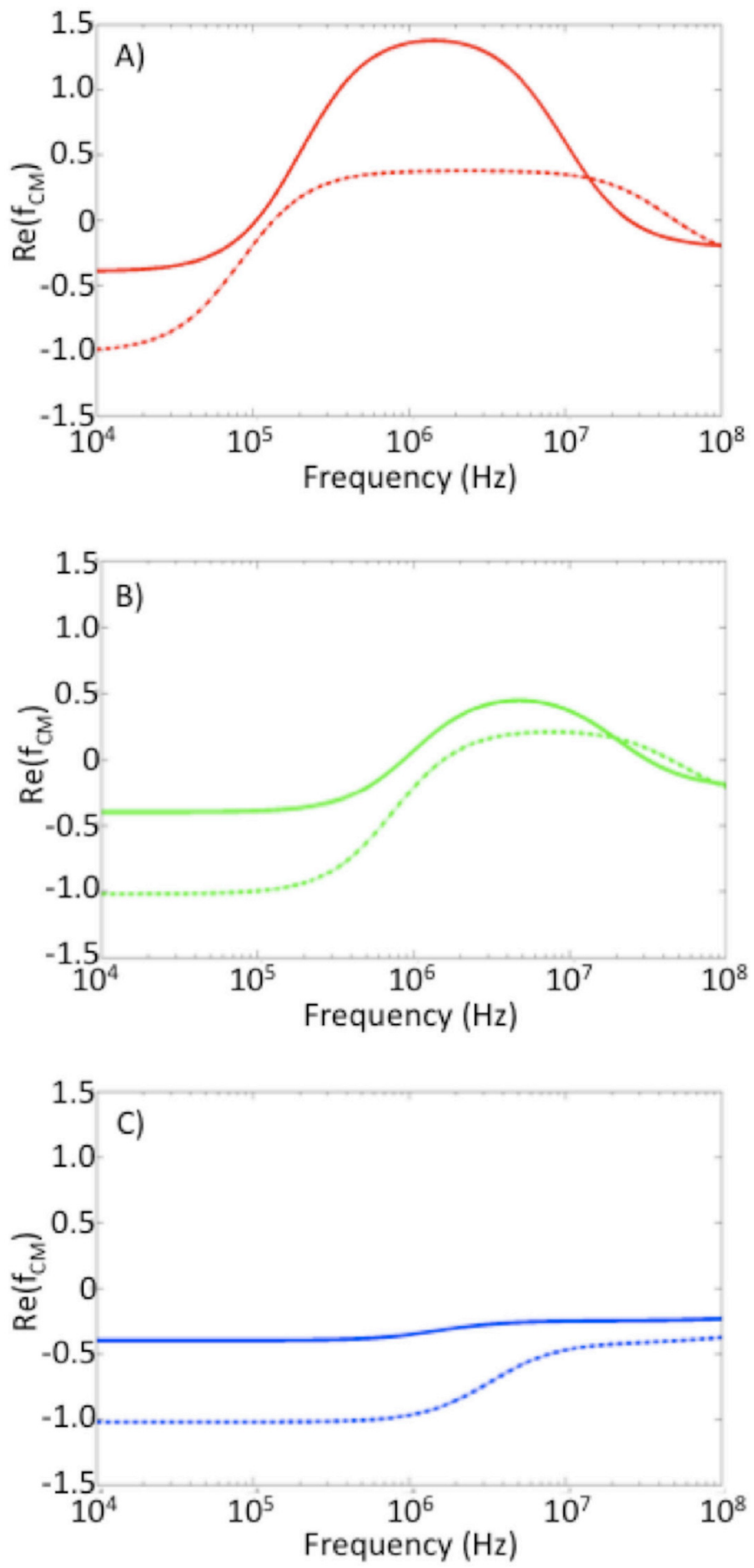

Figure 3.5. Theoretical graphs of major (solid) and minor (dashed) axis ellipsoidal models. Shown for three representative medium conductivities: $0.01 \mathrm{~S} / \mathrm{m}(\mathrm{A}), 0.1 \mathrm{~S} / \mathrm{m}(\mathrm{B})$ and $0.9 \mathrm{~S} / \mathrm{m}(\mathrm{C})$. These conductivities correspond to those used in experimental section. The minor axis hits both COFs at a higher frequency than the major axis. 


\subsubsection{Maxwell-Wagner Membrane Instability}

One of the goals of this work was to integrate the dielectrophoretic force theory with the Maxwell-Wagner membrane instability theory in order to understand why a cell ruptures when subjected to an electric force. When a particle is subjected to an electric field it experiences a force given by:

$$
\vec{F}_{\text {elec }}=q \vec{E}+(m \nabla) \vec{E}+\frac{1}{6} \nabla(\vec{Q}: \nabla \vec{E})+\ldots
$$

The first term refers to the coulombic interaction between the charge of the particle and the electric field. This term vanishes to zero when the particle has no charge or when the time average of the electric field is nearly zero. The other terms come from the polarization of the particle in an inhomogenous field. Therefore, these terms only vanish if the field is homogenous ${ }^{164}$. The relationship between the particle permittivity and the membrane permittivity determines whether the particle experiences positive dielectrophoresis (pDEP) or negative dielectrophoresis (nDEP) as demonstrated in the discussion surrounding Equation (31). A positive $f_{C M}$ is indicative of positive dielectrophoresis and a negative $f_{C M}$ of negative dielectrophoresis. Combining equations 2, 3, and 4 leads to a time-averaged dielectrophoretic force expression:

$$
\rangle \vec{F}_{D E P}\left\langle=2 \pi \varepsilon_{m} R^{3}\left[f_{C M}(\omega)\right] \nabla \vec{E}_{r m s}^{2}\right.
$$

where $\mathrm{E}_{(\mathrm{rms})}$ is the root mean square value of the electric field. This force depends on the real part of the Clausius-Mossotti factor (Equations 31\&36), also known as the in-phase component $^{175}$. This derivation was based on the effective dipole method. A more rigorous way to find the dielectrophoretic force is based on the Maxwell stress tensor method ${ }^{150}$. Both methods result in the equation given above. 
The Maxwell-Wagner effect is a relaxation process that occurs when an electric current must pass through two different dielectrics. Cells usually exhibit this at the interface of their membrane and the cytosol. The basis for this dispersion is that both dielectrics have a conductance in parallel with a capacitance. Therefore, the interface can be charged due to the conductivity ${ }^{180}$. Maxwell derived a solution for the conductivity of a dilute solution of spherical particles based upon $\mathrm{A}, \mathrm{B}$, and $\mathrm{C}$ principles:

$$
\frac{\sigma-\sigma_{m}}{\sigma+2 \sigma_{m}}=\frac{p\left(\sigma_{p}-\sigma_{m}\right)}{\sigma_{p}+2 \sigma_{m}}
$$

where $p$ is the volume fraction of spheres and $\sigma_{\mathrm{p}}$ and $\sigma_{\mathrm{m}}$ refer to the conductivity of the particle and medium, respectively ${ }^{181}$. This theory was expanded by Fricke to include membrane covered spheres where the membrane diameter, $\Delta r$, is much smaller than the particle radius, $r_{p}$ :

$$
\bar{\sigma}_{p}=\frac{\bar{\sigma}_{c}-\left(\frac{2 \Delta r}{r_{p}}\right)\left(\bar{\sigma}_{c}-\bar{\sigma}_{s}\right)}{\left(1+\frac{\Delta r}{r_{p}}\right) \frac{\left(\bar{\sigma}_{c}-\bar{\sigma}_{s}\right)}{\bar{\sigma}_{s}}}
$$

In this expression, $\sigma_{\mathrm{c}}$ is the conductivity of the interior of the sphere and $\sigma_{\mathrm{s}}$ is the conductivity of the membrane ${ }^{182}$. This equation is valid for particles such a red blood cells. The connection between this conductivity expression and the dielectrophoretic force given in Equation (36) with complex permittivity from Equation (43) needs further exploration. 


\subsection{Dielectrophoresis in Microdevices}

In a microfluidic device, particle size determines the feasiblity of dielectrophoretic movement. Particles larger than $1 \mu \mathrm{m}$ experience dominant dielectrophoretic forces and minimal gravitational force and Brownian motion ${ }^{117}$. For human erythrocytes of $7 \mu \mathrm{m}$ diameter, the particle displacement in 1 second is approximately $10 \mathrm{~nm}$ due to the gravitational force and $1 \mu \mathrm{m}$ due to Brownian motion. This dielectrophoretic force displacement can easily dominate these other forces by decreasing the characteristic electrode dimension or increasing the applied voltage across the electrodes ${ }^{117}$. The DEP force is sensitive to particle size, geometry and polarizability (combination of permittivity and conductivity). These versatile equations can distinguish between particles ${ }^{8,17,157,158,160,164,166,167,175,183-186}$, slightly different cell phenotypes ${ }^{12-14}$ and genotypically similar cells either infected versus their equivalent healthy cells ${ }^{3-5}$.

Experiments by Yang and colleagues in $1999{ }^{14}$ and $2000{ }^{13}$ explored dielectrophoresis as a way to separate and identify subpopulations of human leukocytes. Initially, they used electrorotation, which relies upon the imaginary part of the ClaussiusMossotti factor to determine differences between four human leukocytes: T-lymphocytes, B-lymphocytes, monocytes and granulocytes. All cells began with antifield rotation below $6 \mathrm{MHz}$ and experienced cofield rotation above $6 \mathrm{MHz}$, but the cells experienced different peak antifield rotation points: T-lymphocytes $(\sim 350 \mathrm{kHz})$, B-lymphocytes $(\sim 350 \mathrm{kHz})$, monocytes $(\sim 200 \mathrm{kHz})$ and granulocytes $(\sim 300 \mathrm{kHz})$. From the imaginary part of the dielectrophoretic theory equations which govern electrorotation, the following mean membrane capacitance values were calculated: T-lymphocytes $\left(10.5 \pm 3.1 \mathrm{mF} / \mathrm{m}^{2}\right)$, B-lymphocytes $\left(12.6 \pm 3.5 \mathrm{mF} / \mathrm{m}^{2}\right)$, monocytes $\left(15.3 \pm 4.3 \mathrm{mF} / \mathrm{m}^{2}\right)$ and granulocytes 
$\left(11.0 \pm 3.2 \mathrm{mF} / \mathrm{m}^{2}\right)^{14}$. Later work used field flow fractionation dielectrophoresis to separate lymphocytes (both B and T) from monocytes, lymphocytes from granulocytes and monocytes from granulocytes ${ }^{13}$. These works demonstrate dielectrophoresis and electrorotation can separate ${ }^{13}$ and identify ${ }^{14}$ closely related subpopulations of human leukocytes. The concept of using dielectrophoresis to identify and separate subpopulations of human blood cells is further discussed in Chapter 2; concurrently, this information is linked to the known biochemical subpopulation information.

Interesting work in cell dielectrophoresis in microdevices has focused on the separation of healthy and dead/infected cells. Beginning with healthy vs. heat-treated yeast cell studies in 1992, capabilities have increased to achieve detection of leukemia cells from blood cells. Huang and colleagues used frequency changes to move a yeast cell in a polynomial electrode device ${ }^{187}$ and observed differences in electrokinetic behavior between healthy yeast cells and heat treated yeast cells. The core-shell spherical model outline in Section 3.2.2 yielded membrane conductivity increases from $2.5^{*} 10^{-}$ ${ }^{7} \mathrm{~S} / \mathrm{m}$ to $1.6^{*} 10^{-4} \mathrm{~S} / \mathrm{m}$ after heat treatment and interior conductivity decreases from $0.2 \mathrm{~S} / \mathrm{m}$ to $7 * 10^{-3} \mathrm{~S} / \mathrm{m}^{6}$. This work showed that it was possible to experimentally distinguish between live and dead cells, and that the experimental differences could be theoretically tied to physiological changes upon cell death.

Dielectrophoresis has also distinguished healthy erythrocytes from malariainfected erythrocytes ${ }^{4,5}$. Gascoyne and colleagues found that malaria-parasitized cells experience field line inclusion at lower frequencies than healthy erythrocytes; healthy and malaria-infected erythrocytes have different DEP and electrorotation (ROT) spectra. Healthy erythrocytes showed good ROT experiment agreement suggesting membrane 
specific capacitance was $12 \pm 1.2 \mathrm{mF} / \mathrm{m}^{2}$, the cytoplasmic conductivity was $0.52 \pm 0.05 \mathrm{~S} / \mathrm{m}$ and the internal relative permittivity was $57 \pm 5.4^{4}$. These same values were not obtained from the theoretical equations for the infected cells because the experiments showed such large variability, although they did always show deviation from the healthy spectra. The peak for counter-field rotation was at $260 \mathrm{kHz}$ for the healthy cells and around $50 \mathrm{kHz}$ for the infected cells. Also, the magnitude of this rotation was substantially less for the infected cells by $\sim 30$ to $50 \%{ }^{4}$. The DEP spectra also provided insightful information to distinguish healthy versus infected cells by graphing COF as a function of medium conductivity and is reprinted in Figure 2.7. In general, the healthy cells displayed higher cross-over frequencies than their infected counterparts because they are less permeable to the electric field than the infected cells.

Field Flow Fractionation (FFF) combined with $\mathrm{DEP}^{5}$ was able to levitate the healthy/infected erythrocytes at different heights above the electrodes plated on the chamber floor. Since a parabolic flow profile forms, the levitated cells flow through the chamber at a streamline velocity corresponding to their height, resulting in a different elution time based on the healthy/infected status of the cell. The infected cells were levitated more strongly off the chamber floor at the frequencies applied $(40-250 \mathrm{kHz})$ and thereby eluted through the chamber faster ${ }^{5}$. Additionally, leukocytes were separated from both the healthy and infected erythrocytes because they were retained in the chamber and only eluted after the field was turned off. Researchers suggested that this technique could be combined with a cell lysis chamber and a PCR detection stage for onchip malaria detection and analysis ${ }^{5}$. Such a device could make use of the DEP lysis explored in Chapter 4. 


\subsection{Summary}

Various models for dielectrophoretic behavior have been mathematically explored and their influence in dielectrophoretic literature elucidated. The correct model is necessary to yield the most insightful parameters from experimental data in a given system. Selection of the model is based on geometric considerations as well as shell (membrane) structure and thickness compared to the core. This chapter focused on dielectrophoretic behavior for comparison to our experimental results obtained, however the electrorotation behavior (imaginary component of the Clausius-Mossotti factor) is equally important to this field of research. Research measuring both behaviors allows the determination of both components of the dielectric constant: permittivity and conductivity.

A great deal of work has been done to explore the dielectrophoretic behavior of particles $^{8,17,157,158,160,164,166,167,175,183-186}$ and cells ${ }^{1,3-9,12-14,118,123,124,158,159,161,162,188-192}$. In the majority of these works the experimental results have been used to calculate parameters from the governing Clausius-Mossotti factor. This approach is useful because the dielectric parameters of various particle and cell types are estimated. However, at this point, most parameter estimations neglect conductivity to obtain permittivity or viceversa. The resulting values are accurate to the order of magnitude and so there is potential for improvement.

In the work presented in this dissertation, the thin-shell approximation was used with the ellipsoidal model because it was found to best fit the physical parameters of the human erythrocyte as well as the behavior exhibited in DEP experiments. However, this model wouldn't perfectly fit the behavior of the erythrocytes because the geometry is 
only an estimate and the shell is assumed to be homogeneous composition. The strongest evidence of this is that if the model fit perfectly then all human erythrocytes should behave the same regardless of blood type, which is proven to not happen in Chapters 4-6. It is likely that an additional shell is needed that is even smaller than the membrane shell and accounts for the antigenic expression on the blood cell surface. This would take into account differences based on ABO blood type. The difference based on transmembrane Rhesus factor most likely could be dealt with in changing the current membrane dielectric parameters, but because it also functions as an ammonium transporter, the cytosolic parameters may also be affected by the presence or absence of the Rhesus factor.

This chapter provided the foundation, motivation and basis for fitting an appropriate model to experimental data in order to obtain physical dielectric parameters for the cell. In order to match experimental data to theoretical models, four different parameters would need to be adjusted: the conductivity of the core and shell and the permittivity of the core and shell. Since there is only one equation for the dielectrophoretic force, a least squares analysis is the best place to begin fitting these parameters. A Matlab code could be used so that each parameter is individually fit using an iterative loop that performs a least squares analysis to fit the experimental data to the theoretical model by changing only that one parameter. This whole code would need to be iterative such that once all four parameters are fit the code runs again to make sure that each parameter has reached its optimal value. 



\section{Alternating Current Dielectrophoretic Characterization of Human Erythrocyte Lysis in a Medical Microdevice}

\section{1. $\quad$ Abstract}

Medical microdevices, or lab-on-a-chip devices, have the potential to improve the performance, speed and expense of medical tests. Custom fabricated medical microdevices were used to determine the rupturing of human erythrocytes of blood types $\mathrm{A}^{+}, \mathrm{B}+, \mathrm{O}+, \mathrm{AB}^{+}, \mathrm{A}-, \mathrm{O}-$ and $\mathrm{AB}-$ when subjected to a $1 \mathrm{kHz}$ dielectrophoretic field at a constant field density of $0.03 \mathrm{Vpp} /$ micron. Video microscopy images were analyzed to determine a normalized rupturing percentage and thus explore differences in membrane integrity at $1 \mathrm{kHz}$ based on blood type. It was found that $74 \%$ of $\mathrm{B}+$ cells ruptured over the fifteen-minute experiment, more than any other blood type and some blood types did not rupture at all. The B antigen appeared to be especially important by increasing rupturing in the absence of the $\mathrm{A}$ antigen $(\mathrm{B}+$ blood) and inhibiting rupturing in the presence of the $\mathrm{A}$ antigen $(\mathrm{AB}+$ and $\mathrm{AB}-$ blood). The Rhesus factor, a multipass transmembrane protein present in positive blood types also decreased rupturing for each of the four types in the ABO typing system. This dependence could be utilized for differentiation between positive and negative $\mathrm{ABO}$ blood types. The $1 \mathrm{kHz}$ dielectrophoretic microdevice was able to distinguish between $\mathrm{O}^{+}, \mathrm{A}+, \mathrm{B}+, \mathrm{AB}+$ and $\mathrm{A}-$, even taking into account donor and microdevice variations. However, it was not possible to distinguish between blood belonging to groups $\mathrm{A}+$ and $\mathrm{O}-\mathrm{or} \mathrm{O}+$ and $\mathrm{AB}-$ based upon 
the standard $95 \%$ confidence interval (CI). A first-order kinetic model was applied to $\mathrm{A}+$, $\mathrm{A}$ - and $\mathrm{B}+$ yielding reaction rate constants of $0.02 \mathrm{sec}^{-1}, 0.04 \mathrm{sec}^{-1}$, and $0.11 \mathrm{sec}^{-1}$, respectively. This microdevice demonstrated erythrocyte rupture without buffer changes or chemical additives; this technique could be used for cell lysis prior to subsequent subcellular separations and analysis.

\subsection{Introduction}

Lab-on-a-chip devices are widely being explored for rapid, point-of-care (POC) testing for cellular diagnostics adaptable to various medical diseases. These devices could potentially perform complete integration of analytical blood tests with small sample volumes in time-sensitive and resource-limited situations distant from a full medical laboratory. Further, small sample volume, POC devices could rapidly perform cellular and subcellular analysis for constant blood chemistry monitoring of critically ill patients ${ }^{193}$. Human erythrocytes are a convenient cell system because they are readily accessible, are compositionally simpler than other human cells because they lack a nucleus, have phenotypic diversity between donors, and in general provide a wide range of information about a patient's body chemistry and health.

\subsubsection{Erythrocytes}

Red blood cells, also known as erythrocytes are produced and released into the blood stream by the hematopoietic bone marrow as fully mature cells. The life span of erythrocytes in circulation is approximately 120 days. Blood cells perform their primary function of transporting oxygen and carbon dioxide utilizing hemoglobin, a complex protein containing heme groups, within the interior cytosol of the erythrocyte. Human 
erythrocytes are highly deformable biconcave disks approximately 6-8 microns in diameter $^{26}$.

Important medical compatibility factors are determined by the $\mathrm{ABO}$ blood typing system, discovered by Karl Landsteiner in $190{ }^{119}$. This blood typing system is defined by the presence or absence of two main antigens or complex carbohydrates found on the erythrocyte membrane ${ }^{26}$. The number of antigens on the surface of the human erythrocyte varies from person to person but can be as high as 1.5 million. As such, the minor molecular differences between the A and B antigen are amplified on the cell surface $^{122}$. Type A blood is classified by the presence of the A antigen, whereas type B blood is classified by the presence of the B antigen. Type AB blood has both A and B antigens and type $\mathrm{O}$ blood has neither antigen ${ }^{26}$. Another major factor in the ABO system is the presence or absence of antibodies inherently produced in humans against the $\mathrm{ABO}$ antigens that they do not express ${ }^{120}$. Therefore, blood types $\mathrm{A}$ and $\mathrm{O}$ have the $\mathrm{B}$ antibody and blood types $\mathrm{B}$ and $\mathrm{O}$ have the $\mathrm{A}$ antibody. Blood type $\mathrm{AB}$ does not have any antibodies. Because of this unique antigen-antibody relationship, $\mathrm{AB}$ is often referred to as the universal accepter and $\mathrm{O}$ as the universal donor ${ }^{26}$. This relationship is incredibly important for blood transfusions; incompatible blood transfusions can be deadly. In addition to A and $\mathrm{B}$, there is a third antigen, the Rhesus factor, a multipass transmembrane protein ${ }^{120,121,131}$ without an innate antibody, which is important to match during transfusions ${ }^{26}$. If the $\mathrm{Rh}$ antigen is present, the blood type is classified as positive. If the $\mathrm{Rh}$ antigen is absent, the blood type is negative ${ }^{121}$. Rh negative people can synthesize the antibody to Rh positive blood including that of a fetus during pregnancy. The eight resulting blood types are: $\mathrm{A}+, \mathrm{B}+, \mathrm{O}+, \mathrm{AB}+, \mathrm{A}-, \mathrm{B}-, \mathrm{O}-, \mathrm{AB}-$. At the end of 
their life span, the erythrocytes are ruptured by the white blood cells (leukocytes) and removed from circulation by the liver and spleen ${ }^{26}$. The signaling pathway for the lymphocyte-mediated rupture involves surface antigens ${ }^{194}$ and while the normal death of erythrocytes is mediated mostly by loss of membrane lipids and rigidity in the cytoskeleton there are a series of hemolytic disorders mediated by antigen-antibody interactions ${ }^{131}$. Hence the rupturing dependencies observed in this study are physiologically grounded. Since the $\mathrm{ABO}$ antigens reside on and $\mathrm{Rh}$ factors reside in the cell membrane, it is hypothesized that ABO-Rh expression will influence the kinetics of erythrocyte rupture in a low frequency DEP field.

Blood is a convenient diagnostic fluid from which a wide variety of diagnostic tests and disease screening can be run. A complete blood count (CBC) measures cell types, a Basic Metabolic Panel (BMP) measures nutrients and electrolytes in the plasma, and a lipid profile measures cholesterol and triglyceride levels. More specialized medical tests also examine the contents of the desired cell, such as DNA, protein or nucleic acid analysis. Subcellular molecules can only be accessed after cell membrane lysis. This paper reviews chemical, mechanical, and electrical methods of cell lysis and then describes original research into dielectrophoretic red blood cell rupture.

\subsubsection{Chemical Lysis}

Chemical lysis is lysis upon cell contact with a chemical reagent. Chemical cell lysis is the most common method used, however unwanted chemical remain inside the analytical fluid. Erythrocytes can be lysed by hypochlorous acid or at a rate 10-fold faster by hypobromous acid ${ }^{141}$. Vissers and colleagues examined $\mathrm{HCl}$ and $\mathrm{HB}$ treatment effects on thiols, membrane lipids, and membrane proteins to determine the oxidant's 
mechanism. No difference in membrane thiol loss was noted between the two oxidants 141. The hypobromous acid yielded phospholipid bromohydrins reaction products at a concentration of $50 \mathrm{nmol}$ per $10^{7}$ cells. Nearly ten times this amount of hypochlorous acid was needed before any chlorohydrins were detected. At low concentrations, hemolysis was immediate; lipid modification did not occur until much higher concentrations, suggesting that this was not the correct hemolysis mechanism ${ }^{141}$. However, protein modifications in the SDS/PAGE profile were detected at the low concentrations of $20 \mathrm{nmol}$ per $10^{7}$ cells of hypobromous acid and $100 \mathrm{nmol}$ per $10^{7}$ cells of hypochlorous acid. It was concluded that $\mathrm{HCl}$ and $\mathrm{HB}$ modify membrane proteins as their mechanism for erythrocyte lysis ${ }^{141}$. Further, hypothiocyanous acid induced cell death at lower concentrations and with a higher efficiency in murine macrophage cells than hypochlorous or hypobromous acids. This is especially interesting because hypothiocyanous acid is milder acid and is thiol-specific, and impacts intracellular thiols 195. Hypochlorous and hypobromous acid at concentrations of $20 \mathrm{nmol}$ per $10^{7}$ cells lyse 30 and 90 percent of the blood cells respectively in a 15 -minute experiment ${ }^{141,195}$. At concentrations of 25,50 and $100 \mu \mathrm{M}$, hypothiocyanous acid (HOSCN) exhibited a greater percentage of both apoptosis and necrosis of J774A.1 cells over the course of both 1 and 2 hour incubation times than hypochlorous acid ( $\mathrm{HOCl})$ and hypobromous acid (HOBr). In all $100 \mu \mathrm{M}$ tests except the 1 hour apoptosis incubation test this difference in percentage was significant between $\mathrm{HOSCN}$ and $\mathrm{HOCl}$ via a two-way ANOVA test. For all $100 \mu \mathrm{M}$ tests the percentage difference was significant between HOSCN and HOBr via the same test ${ }^{195}$. Necrosis is most often defined by the mechanism of oncosis which is where the cell swells and the membrane breaks down before death ${ }^{196}$. This is the 
mechanism for electroporation-induced cell death. Apoptosis is defined by the cell shrinking and then breaking apart into pieces ${ }^{196}$. The rupture seen in this experimental work likely follows a mechanism of fast oncosis (necrosis) because no cell remnants are seen in the post-rupture microscope images.

Chemical lysis has been demonstrated in five different lab-on-a-chip or microdevice applications ${ }^{197-200}$. One device used picoliter volumes of cells and separately stored the cells and the chemical lysis agent using a liquid-air interface barrier between the two liquid suspensions ${ }^{198}$. The cells and lysing agent were then mixed when the air was pulled out; $3 \mathrm{M}$ guanidine thiocyanate, $0.2 \%$ sodium dodecyl sulfate and $0.1 \%$ sodium dodecyl sulfate were all tested as chemical lysis agents in the device. The lysing agents all had low molecular weights, which lead to rapid diffusion and thus rapid lysis. Protection of the intracellular contents was verified via Oregon-green phalloidin (actin-bound) or YOYO-1 iodide (DNA-bound) fluorescence ${ }^{198}$.

A picoliter sized microwell device used a commercially available lysing detergent CelLytic-M (Sigma-Aldrich) to lyse a phosphate buffer saline diluted cell suspension. ${ }^{199}$. CelLytic-M was designed for protein extraction from mammalian cells for subsequent analytical tests. Picoliters of mammalian cells were lysed in minutes using $70 \%$ CelLytic$\mathrm{M}$; the resulting lysate was analyzed for protein composition and damage. No adverse effects from CelLytic-M were noted in the downstream analysis ${ }^{199}$.Another picoliter microdevice was integrated with micellar capillary electrophoresis with the goal of performing chemical cytometry on the chemical contents of a single cell ${ }^{200}$. The cells were mixed with $20 \mathrm{mM}$ borate buffer, $1 \%$ sodium dodecyl sulfate (SDS) and $30 \mathrm{mM}$ sodium cyanide. The SDS rapidly lysed the cell and the borate buffer shifted $\mathrm{pH}$ to basic 
for further analysis. The cell contents were tagged prior to separation via micellar electrokinetic capillary electrophoresis. This fully integrated chip analyzed one cell in less than one hour ${ }^{200}$. These two systems ${ }^{199,200}$ demonstrate chemical lysis integrated with further single cell analyses.

However, challenges with chemical lysates have been widely noted including dilution of the inner contents of the cell, extended contact time between lysing agent and intracellular contents causing analyte degredation, and the removal of debris after lysis ${ }^{201}$. The unwanted and sometimes harmful chemicals add complexity to devices and remain with the lysate after lysis. Added complexity includes reactant storage, mixing, inherent dilution of the lysate, and chemical removal before on-chip analysis. Microfluidic devices must be robustly designed for these four additional processes and be robust. In microdevices, lysis debris can cause blockages in channels and fouling of channel walls that interfere with fluid flow and subcellular analyte transport thus yielding quality control issues during operation. The use of high potentials was preferred for cell lysis because of safer operation, dual use of the same potentials to separate the lysate ${ }^{201}$. Electrically induced separations are common, so by using mechanical or electrical lysis, the chip is simpler to fabricate/operate and easier to integrate with downstream subcellular anaylsis.

\subsubsection{Mechanical Lysis}

Mechanical approaches can be used to break up the membrane as illustrated in a nano-knives filter to slice the cell membrane of sheep erythrocytes ${ }^{202}$. Rupture was quantified optically from flourescein released from the cell interior. Rapid cell flow through the knives achieved fewer piercings of the membrane, but was equally as 
effective at releasing fluorescein ${ }^{202}$ and thus would give access to intracellular biomolecules. Advantages are that reversible poration may be possible, no chemicals are introduced, and membrane and internal proteins are accessible ${ }^{202}$. However, nano-knives were difficult to fabricate.

\subsubsection{Lysis via Electroporation}

Electrical lysis commonly uses traditional, laser-induced or field-induced electroporation ${ }^{109,203-216}$. Traditional electroporation is the most common electrically driven lysis method, which works via micropore formation in cell membranes.

Electroporation in a capillary electrophoresis environment was achieved in less than $33 \mathrm{~ms}$ after optimizing interelectrode distances and pulse durations ${ }^{203}$. Cell swelling during electroporation and the transfer of membrane impermeable molecules were observed in a high-throughput small lysis section of a microdevice ${ }^{215}$. Cell lysis occurred in $600 \mathrm{~V} / \mathrm{cm}$ or higher electric fields at flow rates between 1.2 and $2.7 \mu \mathrm{L} / \mathrm{min}$ which yielded a cell residence time in the field of approximately $35-95 \mathrm{~ms}$. When the field was $1000 \mathrm{~V} / \mathrm{cm}$ or higher the cells were lysed within $60 \mathrm{~ms}{ }^{215}$.

Capillary electrophoresis was utilized for single cell lysis and analysis in order to discern single specific cell variations in type and composition ${ }^{197}$. Capillary electrophoresis can also be coupled with a pulsed, laser microbeam to lyse cells.

Focusing the laser to a specific location on the cell allowed membrane breakage. Video microscopy recorded cell contents diffuse into solution after rupture. These new ghost cells were then analyzed using capillary electrophoresis ${ }^{213}$.

Human colon carcinoma cells were electroporated between two sawtooth electrodes on either side of a microchannel ${ }^{210}$. Cell viability was determined with 
acridine orange, which stains all cells, and propidium iodide, which cannot cross the membrane of healthy cells. Comparisons with the control group led to the conclusion that electroporation induced release of intracellular contents ${ }^{210}$. Modeling revealed that lower frequencies and higher peak-to-peak voltages result in a higher transmembrane potentials that lead to membrane instabilities and rupture. Direct current electroporation was achieved in an $\sim 500$ to $\sim 62$ microns channel constriction by achieving field variations that mimiced electric pulses as the cell flowed through the device ${ }^{109}$. Devices with multiple narrow sections mimicked multiple pulses to yield significantly better results in transfection. Electroporation is not dimension-dependent so it can be scaled for cell samples from milliliters to picoliters ${ }^{109}$. Dielectrophoretic transport was also observed due to the presence of non-uniform fields in these devices, which could facilitate downstream analysis ${ }^{180}$.

\subsubsection{Lysis via Alternating Current Dielectrophoresis}

The use of cell lysis in microfluidics is not a new research topic ${ }^{217-222}$, however the alternating current dielectrophoresis explored in this work is a novel and convenient method for human erythrocyte lysis because fabrication is simple, no additional reagents are required, and smaller voltages can be employed. Dielectrophoretic forces on cells are due to the cell's frequency-dependent polarizability in spatially nonuniform fields. An added benefit of this dielectrophoretic approach is easier downstream integration with subsequent biochemical analyte transport, separations or focusing with the electric fields. The much smaller fields avoid damage to the internal proteins, heme groups and nucleic 
acids. Thus, this approach enables simpler genomic or proteomic analysis integrated with other lab-on-a-chip processes.

The mechanism of dielectrophoretic lysis at $1 \mathrm{kHz}$, which is in the $\beta$-dispersion region ${ }^{180}$, is likely a Maxwell-Wagner membrane instability. Experiments were run for 15 minutes on custom-fabricated microdevices at $1 \mathrm{kHz}$ and a field density from 0.015 to $0.06 \mathrm{Vpp} /$ micron. Rupture variations were explored within 7 of the $8 \mathrm{ABO}-\mathrm{Rh}$ blood types, microdevice variability, aging of the erythrocytes and washing of the erythrocytes. B- blood was not tested due to its rarity in the population, occurring in only one of fifty people, and the lack of an available donor of this blood type.

\subsection{Materials and Methods}

\subsubsection{Microdevice Fabrication}

Custom fabricated microdevices (see Figure 4.1C\&D) were designed and then fabricated using soft photolithography techniques ${ }^{33,223,224}$. Briefly, silica wafers were cleaned with ethanol, isopropanol and acetone, then functionalized with sodium hydroxide before spincoating negative photoresist to 200 microns. This thickness allowed 100 micron platinum wire to be embedded in the channels. The photoresist was soft baked onto the wafer before exposure to UV light through a 32,521 dpi AutoCAD mask (Fineline Imaging, CO). The UV-polymerized photoresist patterned wafer was then hard baked and put in developer before rinsing away excess photoresist. A 10:1

polydimethylsiloxane (PDMS) elastomer and curing agent (Dow Corning) were poured over the wafer master, degassed 30 minutes and baked overnight at $65^{\circ} \mathrm{C}$. The casting 
was gently pealed off of the master wafer, sample ports were punched with a biopsy

punch and 100 micron platinum wires were fit in a " $\mathrm{T}$ " shape in the casting (Figure 2b).

The PDMS was cleaned with scotch tape and sealed to an isopropanol-washed glass

microscope slide by heating in a $95^{\circ} \mathrm{C}$ oven for 10 minutes. The device was tested for

sealing and the electrode distance verified to be between 100 and 300 microns.

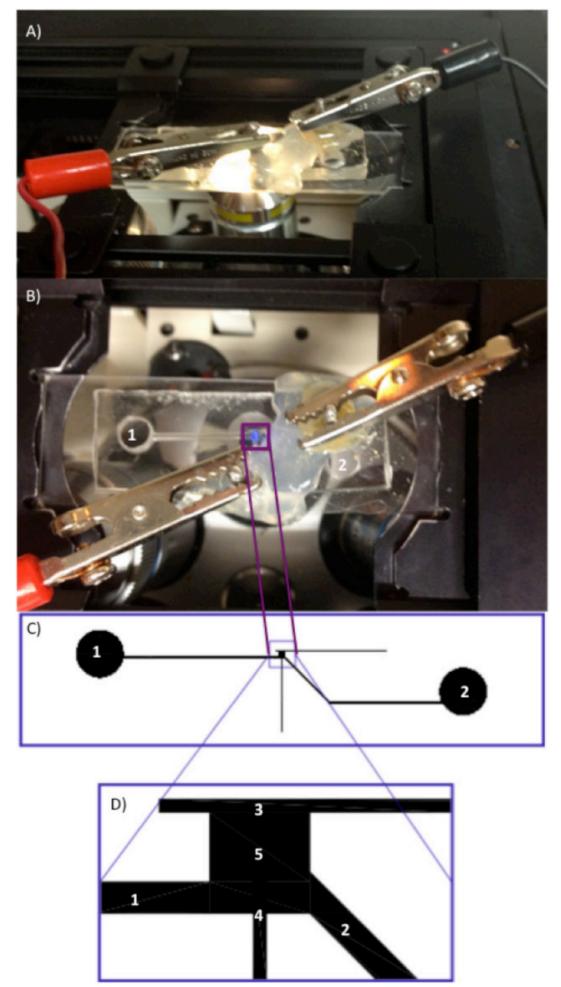

Figure 4.1. Side (A) and Top (B) views of the microdevice on the microscope stage with alligator clips leading to AC Generator. AutoCAD mask of complete fluidic layer (C) and magnified view of the lysis chamber (D). Labels are 1: Inlet, 2: Outlet, 3: Horizontal grounded electrode, 4: Vertical active electrode and 5: lysis chamber.

\subsubsection{Erythrocyte Sample Preparation}

Whole blood samples were obtained from anonymous donors with blood type proof via venipuncture at the Longest Student Health Center at Mississippi State University. Blood volumes of $4 \mathrm{~mL}$ were drawn into vacuutainers with $1.8 \mathrm{mg} \mathrm{K} \mathrm{K}_{2}$ EDTA 
anticoagulant per $\mathrm{mL}$. Blood was stored at $5^{\circ} \mathrm{C}$; experiments occurred on the same day as donation. Donor variation was tracked by having one donor for each blood type donate again five days later. Variations between two microdevices were tested within a single donation. Whole blood was centrifuged at $1400 \mathrm{rpm}$ for 4 minutes to separate cells from the plasma. For the experiments determining the affect of washing on erythroyctes, the cells were resuspended in isotonically matched phosphate buffer saline (PBS) and then re-centrifuged, with this process repeating four times. The erythrocyte fraction was then mixed 1:750 with the same PBS and loaded into the custom fabricated microdevice. For the Concentration Dependency tests this volume-to-volume ratio was also adjusted to $1: 1500,1: 3750$ and $1: 7500$.

\subsubsection{Experimental Procedure}

As shown in Figure 4.1, the microdevice was hooked up the alternating current generator such that the horizontal electrode was always grounded and the vertical electrode is always active. The verified electrode separation distance determined the applied peak-to-peak voltage via the following equation:

$$
E=V / d
$$

The electric field, E, was fixed to 0.015, 0.03 (base rate for all experiments except Field Strength Dependency tests) or $0.06 \mathrm{Vpp} /$ micron via a waveform generator at $1.0 \mathrm{kHz}$ for 15 minutes. In order to have an accurate initial cell count, the first image was always taken before the field was turned on. Images were taken every 15 seconds for 15 minutes with the Zeiss Axiovert 200M inverted light microscope (Zeiss). For the Frequency 
dependency tests, images were taken every 15 seconds for 50 minutes allowing for an exposure time of 10 minutes for each frequency from $1-10 \mathrm{kHz}$. The various dependencies tested along with the experimental conditions of blood type, field strength, washing, concentration of cells to PBS and frequency are outlined in Table 4.1. 
Table 4.1.

Experimental Plan for antigen, field, washing, concentration, and frequency dependencies tested. Columns refer to individual experiments and rows are experimental parameters within that experimental set. Bold parameter is the parameter of interest for that set of experiments.

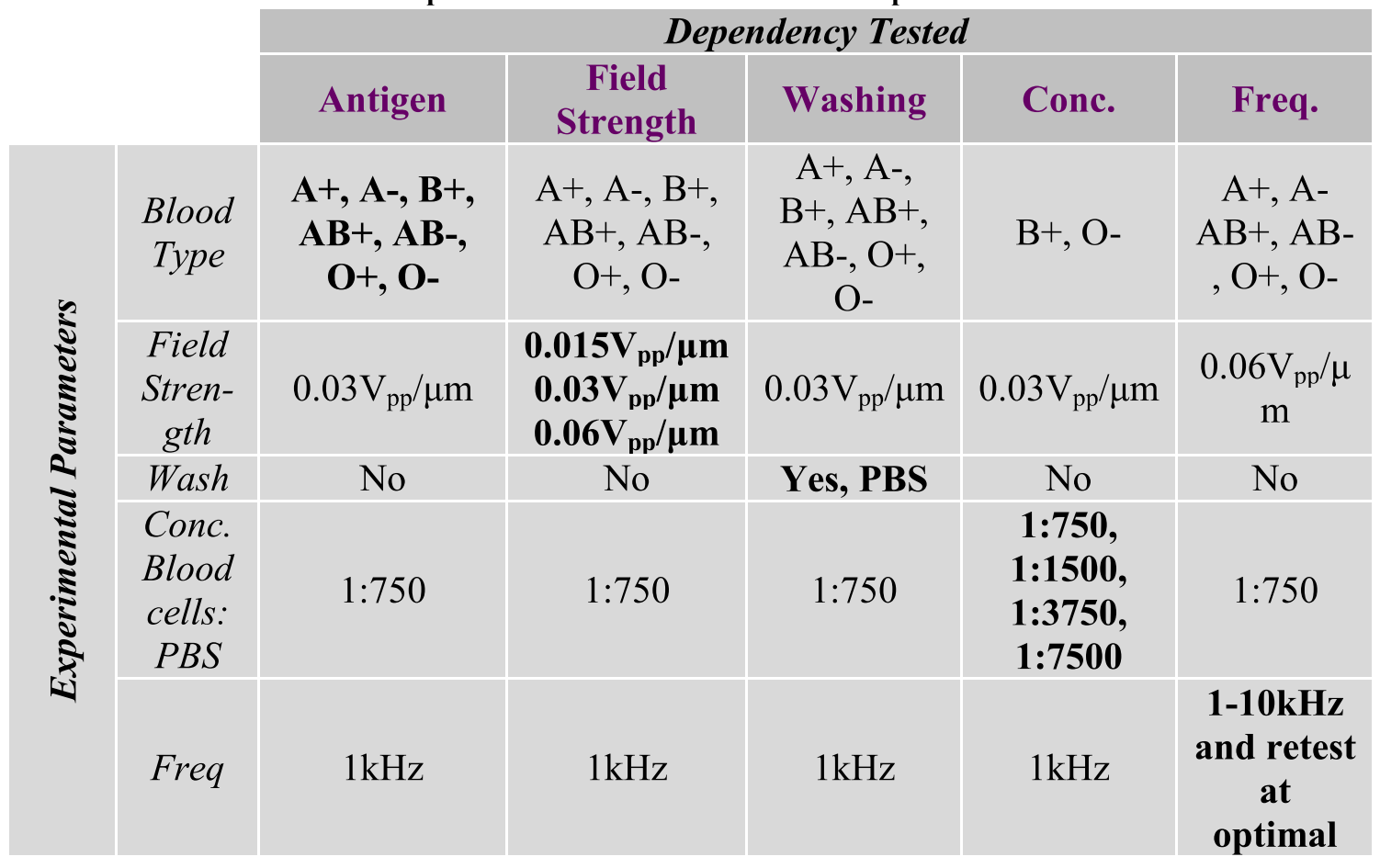

\subsubsection{Data Analysis}

Cells were manually counted and the fraction, $\mathrm{X}_{\mathrm{RBC}, \mathrm{t}}$, calculated with time as follows:

$$
X_{R B C, t}=\frac{N_{0}-N_{t}}{N_{0}}
$$

The number of erythrocytes at time zero, $\mathrm{N}_{0}$, were quantified before electric field application. Overall rupturing profiles, $\mathrm{X}_{\mathrm{RBC}, \mathrm{t}}$, for manual and computer analysis were compiled from cell counts at each time, $\mathrm{N}_{\mathrm{t}}$. Computer facilitated cell counting was accomplished in Zeiss' AxioVision 4.6 Automeasurement Program. Object recognition algorithms were run with user-control over contrast, grayscale pixel recognition for the 
cell edge, permissible size range, and manual segmentation of large cell clusters. Algorithm outputs included cell counts, cell size, and x/y position. Program accuracy was explored by comparing manual and computer-generated counts in overall rupture profiles. Computer rupture profiles were used when the $t=15$ min count was within $10 \%$ of manual count. The final rupture fraction was calculated as

$$
X_{f}=\frac{N_{0}-N_{15}}{N_{0}}
$$

where $\mathrm{N}_{15}$ is the number of cells that are viable at the end of the 15 minute experiment. The $95 \%$ confidence intervals were calculated from the computer counts by first averaging the rupture fraction for each time point across the repeats for that blood type and then taking the confidence interval for each time point. Final rupture and $95 \%$ confidence intervals for the final rupture fraction were used to complete pair-wise comparisons between blood types.

The rupturing of the blood cells can also be treated as a first order reaction where the species of interest is the number of unruptured blood cells. The rate equation for this situation is:

$$
\ln \left[N_{t}\right]=-k t+\ln \left[N_{0}\right]
$$

When the natural logarithm of the number of unruptured cells is plotted versus time for each of the blood types, the resulting linear fit would have a slope corresponding to the rate constant $\mathrm{k}$ and a y-intercept of the natural logarithm of the original number of unruptured cells. 
Another way to determine the rate constant is to plot $\mathrm{N}_{\mathrm{t}} / \mathrm{N}_{0}$ which corresponds to the adjusted rate equation:

$$
\frac{N_{t}}{N_{0}}=e^{-k t}
$$

In order to determine the rate constant, $\mathrm{k}$, the ratio of $\mathrm{N}_{\mathrm{t}} / \mathrm{N}_{0}$ was plotted versus time for all blood types and Excel was used to fit the data to an exponential decay equation.

\subsection{Results and Discussion}

The Rhesus factor and ABO antigen dependency of rupturing were determined as well as variation within donor, variation within device, aging of the erythrocyte sample, field strength dependency, the effect of washing erythrocytes with phosphate buffer saline prior to experimentation and the effect of changing frequency on the rate of rupture. Ruptured erythrocytes were tabulated and overall rupture profiles were compiled using Equation 2 for blood types $\mathrm{A}+, \mathrm{B}+, \mathrm{O}+, \mathrm{AB}+, \mathrm{A}-, \mathrm{O}-$ and $\mathrm{AB}-$. Representative profiles are shown for B+ blood in Figure 4.2A\&B. Initial cell counts ranged from 82 to 1413 and final cells counts ranged from 44 to 2148 . Average rupturing profiles, and 95\% CIs for $3 \leq \mathrm{n} \leq 6$ repeats were calculated for each dependency. Due to the availability of blood donors, experimental n-numbers for all blood types were: $A+=5, A-=6, B+=4, A B+=4$, $\mathrm{AB}-=3, \mathrm{O}+=6$ and $\mathrm{O}-=5$. 

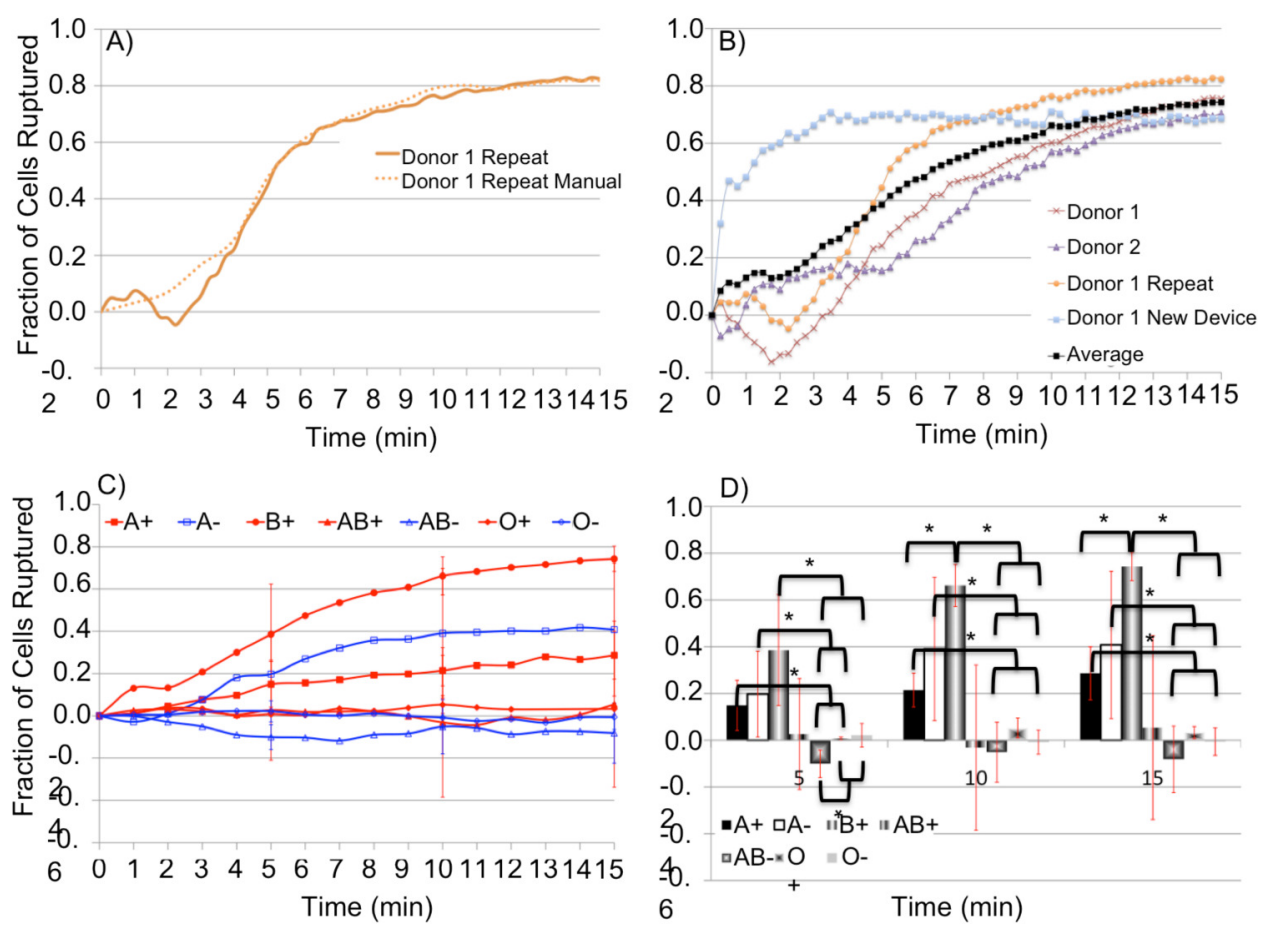

Figure 4.2. Time dependent rupturing for $B+$ manual versus computer (A), all computer-analyzed B+ donors (B) and the average profile for all blood types tested (C). Shown in (D) is the fraction of cells ruptured at 5, 10 and 15 minutes along with $95 \%$ confidence intervals. Statistically relevant differences at a $95 \%$ CI are indicated by *.

Rupture profiles provided insight into time dependence of cell rupturing in the dielectrophoretic fields. Average profiles with the associated $95 \%$ CIs were the basis for comparison between each dependency. Figure 4.2 shows the progression of rupture profiles for $\mathrm{B}+$ manual count comparison with computer (B) to solely computer with the average across all experiments (D). Figure 4.2A demonstrates the agreement between manual and computer generated rupture profiles for Donor $1 \mathrm{~B}+$ repeat. For each of the seven blood types tested the overall rupture profile of erythrocytes was calculated and graphed. The blood types were tested for dependency on both the Rhesus factor antigen as well as the $\mathrm{ABO}$ antigen. They were also test for variation between donors, variation 
within a single donor across two donations and variation within a single donation across two microdevices.

Figure 4.2B compares computer generated rupturing profiles for different $\mathrm{B}+$ donors (lines are present only to guide the eye); an average (black points) profile for each blood type was calculated. Closer examination of the $\mathrm{B}+$ Donor 1 and its repeat demonstrates a positive DEP concentrating effect whereby cells move into the field of view for the first $\sim 2$ minutes followed by cell rupture. Positive DEP concentrating was blood type dependent; for AB-, this happened consistently yielding negative rupture fractions. Average rupturing profiles are in Figure 4.2C for seven blood types and Figure 2D shows the $95 \%$ CI bars for 5, 10 and 15 minutes into the experiment. Confidence intervals were calculated for each timepoint across a dependency as described in materials and methods.

The Rhesus factor appears to have a possible influence on the overall rupturing profile. Comparisons between the positive and negative blood types, reveals that the presence of the Rhesus factor transmembrane protein seems to inhibit rupturing in three of the four groups $(\mathrm{O}, \mathrm{A}$ and $\mathrm{AB})$. This trend could not be verified for $\mathrm{B}$ because $\mathrm{B}-$ donors were unavailable.

Comparison within a Rhesus group, i.e. the positive blood types, suggests it is possible to distinguish at $95 \%$ confidence between the four blood types. In Figure 4.2D, distinction between overall rupture fractions is possible as early as 5 minutes into the experiment; it is possible to distinguish $\mathrm{B}+$ from $\mathrm{AB}-, \mathrm{O}+$ and $\mathrm{O}-$ at a $95 \% \mathrm{CI}$ although this changes. After 5 minutes, AB-, O+ and O- cannot be distinguished with $95 \%$ confidence. At 10 minutes, it is also possible to distinguish $\mathrm{A}+$ and $\mathrm{A}-$ from $\mathrm{AB}-, \mathrm{O}+$ 
from $\mathrm{O}-$, and $\mathrm{A}+$ from $\mathrm{B}+$ at $95 \%$ confidence. $\mathrm{A}-$ cannot be distinguished from either $\mathrm{A}+$ or $\mathrm{B}+$, nor can $\mathrm{AB}+$ be distinguished from the other blood types because of large rupture profile variations. Type B+ blood cells rupture $74 \%$ in 15 minutes, more than any other blood type. Approximately $29 \%$ of A+ cells rupture in $15 \mathrm{~min}$ and are distinguishable at $95 \%$ confidence from both $\mathrm{B}+$ and $\mathrm{O}+$ cells that rupture only $3 \%$. An average of $5 \%$ of $\mathrm{AB}+$ cells rupture, but a large deviation exists in the data due to one peculiar experiment exhibiting strong positive DEP without rupture. This data could not be excluded statistically with a Q-test at $90 \%$ CI, nor could it be excluded because of an identifiable experimental mistake.

Comparison within the three negative blood types tested suggests the $\mathrm{ABO}$ antigen expression can be distinguished at a confidence interval of less than $95 \%$. It can also be seen that at different time points throughout the 15 minute experiment it is possible to distinguish between blood types. Blood of type A- final rupture values indicate a higher percentage (41\%) than both $\mathrm{O}-(-0.5 \%)$ and $\mathrm{AB}-(-8.1 \%)$. This Adifference is statistically significant at a 95\% confidence as seen in Figure 4.2D. Although there is not a statistically significant difference between $\mathrm{O}$ - and AB-, the experiments behave differently which is apparent when comparing the profiles or 5,10 , and 15 minute confidence intervals. O- cells moved very little and did not rupture, whereas AB- cells concentrated into the field of view under pDEP behavior with minor rupture events.

A trend comparison was conducted between the positive and their negative blood types. Figure 4.2D shows that for the positive blood types the rupture fraction increases from $\mathrm{O}$ to $\mathrm{A}$ to $\mathrm{B}$ and then $\mathrm{AB}$ does not rupture at all. For the negative blood types the 
rupture fraction increases from $\mathrm{O}$ to $\mathrm{A}$ and $\mathrm{AB}$ does not rupture at all $(\mathrm{B}$ is not included due to donor logistics). For each of the blood types, the negative counterpart has a higher final rupture fraction than the positive counterpart. Assuming a consistent trend between Rhesus positive and Rhesus negative cells, the final rupture percentage of B- is expected to be over $74 \%$.

In summary, $\mathrm{B}+$ cells rupture more readily than any other blood type ending at $0.74 \pm 0.06$ rupture fraction. $\mathrm{A}+$ and $\mathrm{A}-$ also have a propensity to rupture at $0.29 \pm 0.11$ and $0.41 \pm 0.31$ final rupture fractions, respectively. Blood types $\mathrm{O}+, \mathrm{O}-$, and $\mathrm{AB}+$ do not experience appreciable rupturing, while $\mathrm{AB}$ - blood cells remain intact and experience positive DEP concentration up the electric field gradient at $1 \mathrm{kHz}$. The final rupture fractions for each donor and for all blood types are compiled in Table 4.2. From these experiments, a series of analysis of variance (ANOVA) tests were run using Excel 2010. The first test run was a single-factor ANOVA with the null hypothesis that the final rupture fraction is the same for all blood types test at an alpha level of 0.05 , which corresponds to a confidence interval of $95 \%$. The $\mathrm{F}_{\text {statistic }}$ value for this ANOVA test was 6.37 and the $F_{\text {critical }}$ was 2.47. Since the $F_{\text {statistic }}$ value is greater than $F_{\text {critical }}$ the null hypothesis is rejected, meaning that the mean final rupturing fraction is not the same for all blood types at an alpha level of 0.05 . This confirms that the select blood types can be distinguished from others at a 95\% confidence level. This test does not tell us which blood types those are. In order to gather that information the different combinations of groups of blood type would have to be tested against one another and significant differences sought. 
Table 4.2.

Final rupturing fraction for seven blood types tested and the four donors with the repeat donor and the repeat donor with a new device. Missing cells correspond to tests that either couldn't be run or were deemed statistical

\begin{tabular}{|l|c|c|c|c|c|c|c|}
\hline & A+ & A- & B+ & AB+ & AB- & O+ & O- \\
\hline Donor 1 & 0.459 & 0.170 & 0.756 & 0.268 & -0.226 & 0.136 & 0.093 \\
\hline Donor 2 & 0.367 & 0.053 & & -0.520 & -0.006 & 0.099 & -0.092 \\
\hline Donor 3 & 0.232 & 0.016 & 0.704 & 0.380 & & 0.184 & \\
\hline Donor 4 & & 0.823 & & 0.091 & & -0.027 & -0.027 \\
\hline Donor 1 Repeat & 0.124 & 0.921 & 0.824 & & & -0.007 & -0.017 \\
\hline Donor 1 Device & 0.250 & 0.463 & 0.687 & & -0.012 & -0.189 & 0.015 \\
\hline
\end{tabular}

A kinetic reaction model was applied to the overall rupture profiles. Equation (3) reliably fit the data for $\mathrm{A}+, \mathrm{A}-$ and $\mathrm{B}+$ as demonstrated in Figure 4.3. Table 4.3 compiles the reaction rate equation and the exponential constant, $\mathrm{k}$, which corresponds to the reaction rate. $\mathrm{B}+$ erythrocytes rupture nearly 3 times faster than $\mathrm{A}$ - and 5 times faster than $\mathrm{A}+$. Propagation of error from the original confidence intervals reveals that the rate constants are fairly consistent within a $95 \% \mathrm{CI}$, especially for $\mathrm{A}+$ and $\mathrm{B}+$, as shown in Table 4.3. The confidence interval is of the same order of magnitude as the rate constant for A- because A- cells appeared to display three distinct behaviors. Three donors experienced negligible rupturing, while the donor repeat and a fourth donor experienced substantial rupture all in the same device. The donor repeat in a new device displayed an intermediate amount of rupture. Thus, a statistical outlier could not be identified and systemic or procedural were not noted. 
Table 4.3.

Kinetic reaction models and rate constants for $\mathrm{A}+, \mathrm{A}-$ and $\mathrm{B}+$, which experienced enough rupture to fit to a reaction model.

\begin{tabular}{|c|c|c|}
\hline Blood Type & $\mathbf{N}_{\mathbf{t}} / \mathbf{N}_{\mathbf{0}}=$ & $\mathbf{k}=$ \\
\hline $\mathrm{A}+$ & $0.98 \mathrm{e}^{-0.02 \mathrm{t}}$ & $0.02 \pm 0.002 \mathrm{sec}^{-1}$ \\
\hline $\mathrm{A}-$ & $1.00 \mathrm{e}^{-0.04 t}$ & $0.04 \pm 0.04 \mathrm{sec}^{-1}$ \\
\hline $\mathrm{B}+$ & $1.06 \mathrm{e}^{-0.11 \mathrm{t}}$ & $0.11 \pm 0.008 \mathrm{sec}^{-1}$ \\
\hline
\end{tabular}

Rupturing kinetics for each blood type begins to explore the time dependent electrokinetic instabilities of the cell membrane and could eventually enable a priori selection of rupture percentage as a function of time and electric field. These preliminary results suggest this could be used as a blood type determinant and suggest that additional research into the physiological reason for these differences should be conducted.

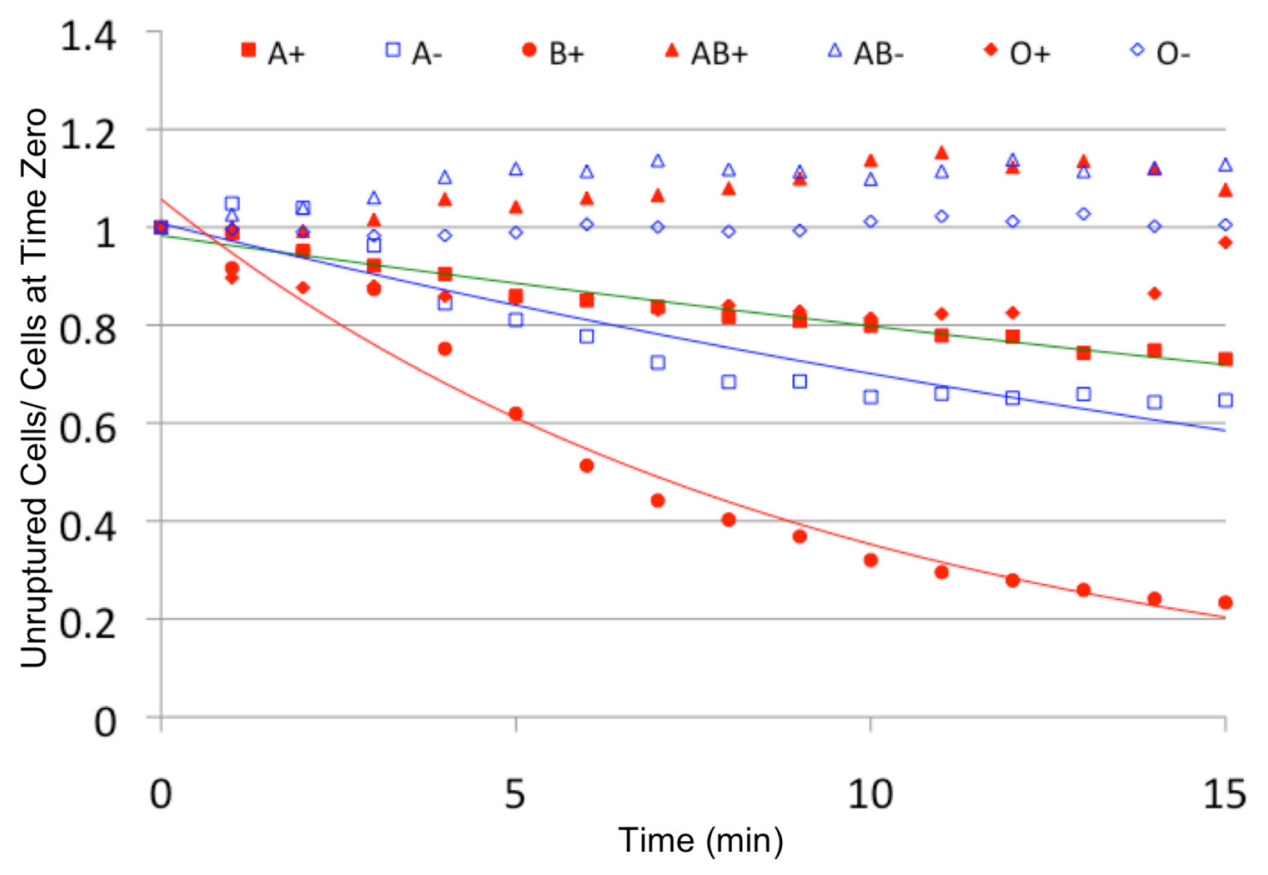

Figure 4.3. Graph showing kinetic fit for $A+(n), A-(\square)$ and $B+(\bullet)$ based on Equation (3). The erythrocytes are assumed to follow a first order reaction rate where the species of interest is the number of unruptured blood cells versus time.

\subsubsection{Variation Within Donor}

One donor for each blood type donated on two separate occasions to explore variation within a single donor. Table 4.4 presents small same donor variations for all 
blood types except A- (0.282). Lowest variations between donations were observed with $\mathrm{B}+$ and $\mathrm{O}-$ at 0.002 and 0.006 , respectively. $\mathrm{A}+$ and $\mathrm{O}+$ have an order of magnitude greater variation between donors at 0.01 and 0.056 , respectively. Donor 1 repeat for $\mathrm{AB}+$ was excluded for both the old and new device because video contrast prevented analysis. Donor 1 Repeat for AB- was excluded via a 99\% Q-test. Two-factor without replication ANOVA tests were run to determine if differences between same donor repeats in Table 4.4 were statistically significant at $95 \%$ confidence. The null hypothesis was the mean final rupture fraction between Donor 1 and Donor 1 repeat was not different at an alpha level of 0.05 . The $\mathrm{F}_{\text {statistic }}$ value was 0.06 and less than $\mathrm{F}_{\text {critical }}$ was 7.71 , so the null hypothesis is accepted, meaning there is no statistically significant difference between Donor 1 and Donor 1 Repeat at a 95\% confidence level across all blood types.

\subsubsection{Variation Between Microdevices}

The custom fabricated microdevices had larger tolerances than traditional clean room microfabricated devices, so overall rupturing profile variations were examined between devices. The same donor was tested on one device that had $6+$ hours while the second device had 0 experimental hours. Blood type $\mathrm{O}-$ had the lowest variation between its first and second device at 0.001 . Blood types $\mathrm{A}+, \mathrm{B}+$ and $\mathrm{O}+$ had devices with variations ranging from 0.008 to 0.017 and $\mathrm{A}$ - had the highest variation between devices with 0.105. Two-factor without replication ANOVA tests were conducted to determine if the null hypothesis that the mean final rupturing fraction between Donor 1 Repeat and Donor 1 Device shown in Table 4.4 was not different at an alpha level of 0.05 . The

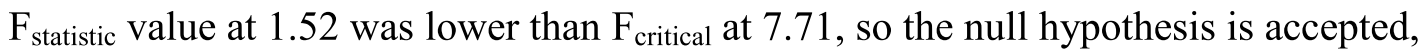
meaning there is no statistically significant difference between the custom built devices at 
a $95 \%$ confidence level across all blood types. Microdevice variations could be due to changing chemical and morphological surface of the platinum wires ${ }^{225}$ or slight geometrical differences. The key implication from this analysis is that device variation was not a factor in the blood type differences observed in Figure 4.2 and Figure 4.3.

Table 4.4.

Final rupturing five blood types that had donor and device dependency tested as well as the variation between the two donations from the same donor and the two devices from the same day of donation.

\begin{tabular}{|c|c|c|c|c|c|}
\hline & $\mathbf{A}+$ & $\mathbf{A}-$ & $\mathbf{B}+$ & $\mathbf{O}+$ & $\mathbf{O}-$ \\
\hline Donor 1 & 0.459 & 0.170 & 0.756 & 0.136 & 0.093 \\
\hline Donor 1 Repeat & 0.124 & 0.921 & 0.824 & -0.007 & -0.017 \\
\hline Donor 1 Device & 0.250 & 0.463 & 0.687 & -0.189 & 0.015 \\
\hline $\begin{array}{c}\text { Variation between Donation } \\
\text { Days }\end{array}$ & 0.056 & 0.282 & 0.002 & 0.010 & 0.006 \\
\hline $\begin{array}{c}\text { Variation between Devices } \\
\text { on same Donation Day }\end{array}$ & 0.008 & 0.105 & 0.009 & 0.017 & 0.001 \\
\hline
\end{tabular}

Results demonstrated erythrocyte rupturing in an alternating current dielectrophoretic microdevice is blood type dependent, with $\mathrm{B}+$ rupturing more cells than any other blood type. Variations from 0.001 to 0.105 occurred between different microdevices with a single donor and from 0.002 to 0.282 between same donor, different donation days on the same microdevice.

\subsubsection{Erythrocytes Aging in Storage}

A longitudinal study was conducted to track overall rupturing profiles for five blood types for six days following donation. Individual single factor ANOVA tests were run for each of the blood types aged: $\mathrm{A}+, \mathrm{B}+, \mathrm{AB}+, \mathrm{O}+$ and $\mathrm{O}-$. The null hypothesis for all of these tests was that aging does not affect the mean overall rupture fraction at an alpha of 0.05 and the results are shown in Table 4.5. F values less than $F_{\text {critical }}$ mean that 
overall rupture fraction did not change with age for that blood type at a 95\% CI. Blood types $\mathrm{A}+($ only tested up through Day 4$), \mathrm{B}+, \mathrm{AB}+$ and $\mathrm{O}+$ did not change with age. $\mathrm{O}-$ was the only blood type to show that an age dependency on erythrocyte rupture. Figure 4.4A demonstrates that overall $\mathrm{O}$ - decreases in overall rupture fraction over 5 days in storage. A+ experienced a notable decrease after the day of donation (Day 0) but rupture behaviors remained steady thereafter. The other blood types showed slight increases in overall rupture fraction with storage, but none of these changes were significant at $95 \%$ confidence.

Table 4.5.

Single factor ANOVA for erythrocyte storage age dependency. $F$ and $F_{\text {critical }}$ values were used to determine validity of the null hypothesis at an alpha of 0.05 . Green shading indicates tests where age did not alter final rupture values while red shading at a $95 \%$ confidence level.

\begin{tabular}{l|c|c|c|r|r|r|} 
& $S S$ & $d f$ & $M S$ & $F$ & P-value & $F$ crit \\
\hline A+ & 0.12 & 2 & 0.06 & 0.82 & 0.47 & 4.26 \\
B+ & 0.04 & 3 & 0.01 & 2.48 & 0.11 & 3.49 \\
AB+ & 0.27 & 3 & 0.09 & 1.03 & 0.41 & 3.49 \\
O+ & 0.11 & 3 & 0.04 & 1.39 & 0.31 & 4.07 \\
\hline O- & 0.50 & 3 & 0.17 & 6.79 & 0.01 & 3.49
\end{tabular}

\subsubsection{Field Strength Dependency}

Dielectrophoretic forces on cells increase with increasing applied field strength as shown in Chapter 3, Equation (36). Thus, overall rupture profiles were examined at 0.015, 0.03 , and $0.06 \mathrm{~V} \mathrm{pp}_{\mathrm{pp}} / \mu \mathrm{m}$ to determine if increasing DEP forces on the cells would result in increases in cell rupture across all blood types. Two-factor without replication ANOVA was run with the null hypothesis that the overall rupture fraction would not change with field strength. The resulting $F$ value of 7.66 was greater than the $F_{\text {critical }}$ value of 5.14 indicating a statistically significant difference in overall rupture fraction for all blood types at an alpha of 0.05 . Figure $4.4 \mathrm{~B}$ demonstrates an increasing trend in rupture fraction 
with increasing field for $\mathrm{A}^{+}, \mathrm{B}+, \mathrm{AB}+$ and $\mathrm{O}^{+}$. Experiments were not repeated, so further statistical analysis and $95 \%$ CIs were not calculated.

\subsubsection{Washing Dependency}

A plethora of antibodies, proteins and biochemical indicator molecules exist in the blood plasma surrounding blood cells. To determine whether cell interactions with the plasma such as antigen-antibody interactions ${ }^{1}$ impacted dielectrophoretic rupture behaviors, cells were washed with isotonic phosphate buffer saline prior to $1 \mathrm{kHz}$ DEP rupture treatment. Final rupture fractions for washed and unwashed cells are plotted with their 95\% CI bars in Figure 4.4C. A two-factor with replication ANOVA test yielded a F value of 0.32 , which is less than $F_{\text {critical }}$ of 4.20 indicating no statistical difference between simple dilution or washing followed by dilution for any blood type. Washing thus adds an unnecessary and complicated step to a future microdevice. These results also suggest that plasma biomolecules are not involved in membrane rupture mechanisms.

\subsubsection{Concentration Dependency}

Cells were diluted with isotonic phosphate buffer saline from whole blood as described in materials and methods. To determine if cell/cell interactions influenced dielectrophoretic rupture behaviors, two blood types, $\mathrm{B}+$ and $\mathrm{O}-$, representing the highest and lowest rupture fractions, were tested at 1:750, 1:1500, 1:3750 and 1:7500 cells to PBS as seen in Figure 4.4D. Single factor ANOVA tests were run separately for B+ and $\mathrm{O}$ - and indicated that the overall rupture fraction did not change with concentration at an 
alpha of 0.05 . For $\mathrm{B}+$ the $\mathrm{F}$ value was 2.07 , less than the $\mathrm{F}_{\text {critical }}$ value of 2.79 , and for $\mathrm{O}-$ the $F$ value was 0.52 , less than the $F_{\text {critical }}$ value of 2.77 . Large variations existed between experimental repeats as demonstrated by the large $95 \%$ CIs, thus it is inconclusive whether cell/cell interactions impact the field induced membrane instabilities leading to membrane rupture. Further the notion that a critical cell/cell spacing could achieve polarization shielding and thus stabilization of the membrane is not supported by the data.

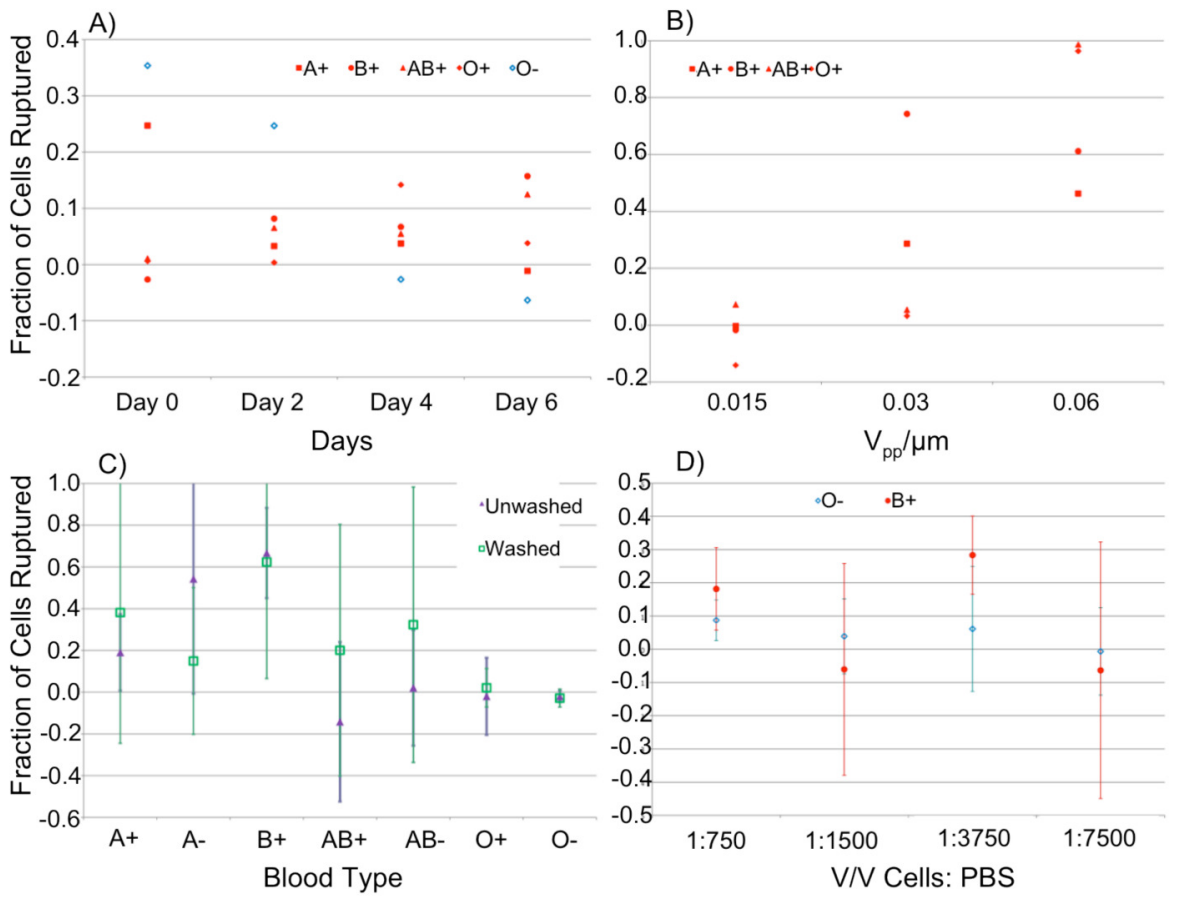

Figure 4.4. Final cell rupture fraction, $X_{f}$, for the dependencies: Erythrocyte storage age (A), Electric field strength (B), Washing, (C) and Cell concentration (D). For clarity, $95 \%$ confidence bars were calculated but are not shown for storage age (A) and field strength (B). Symbols for (A), (B) and (D) are: A+ (n), B+(•), AB+( $(\notin)$, $O+(u)$ and $O-($ ). Bars in (C) and (D) represent $95 \%$ confidence intervals.

\subsubsection{Frequency Dependence}

To determine if different blood types exhibited different optimal frequencies for cell rupture, frequency dependency studies between 1 and $10 \mathrm{kHz}$ were completed. The goal was to achieve maximum rupture fractions and to discern if $\mathrm{ABO}-\mathrm{Rh}$ expression influenced the DEP facilitated membrane rupture. Previous work by this group examined 
dielectrophoretic frequency dependence of $\mathrm{ABO}-\mathrm{Rh}$ erythrocytes at higher frequencies $1,3-9,12-14,118,123,124,158,159,161,162,188-192$. Only a single donor and donation was tested for A+, $\mathrm{A}-, \mathrm{AB}+, \mathrm{AB}-, \mathrm{O}+$ and $\mathrm{O}-$, which limited statistical analysis for this dependency. Figure 4.5 shows frequency dependency by blood type: Maximum final rupture fractions were observed for $\mathrm{A}+$ at $4 \mathrm{kHz}, \mathrm{A}-$ at $6 \mathrm{kHz}, \mathrm{AB}+$ at $1 \mathrm{kHz}, \mathrm{AB}-$ at $6 \mathrm{kHz}, \mathrm{O}+$ at $2 \mathrm{kHz}$ and $\mathrm{O}-$ at $3 \mathrm{kHz}$. For all three ABO pairs tested, the blood type (negative) that does not express the Rhesus factor hits its maximum rupturing fraction at a higher frequency than the equivalent positive blood type. This is further evidence that the $\mathrm{ABO}$ antigens, as well as the transmembrane Rhesus factor protein, affect the dielectrophoretic behavior of human erythoryctes. Further work needs to be done in this area to determine the statistical significance of these differences.

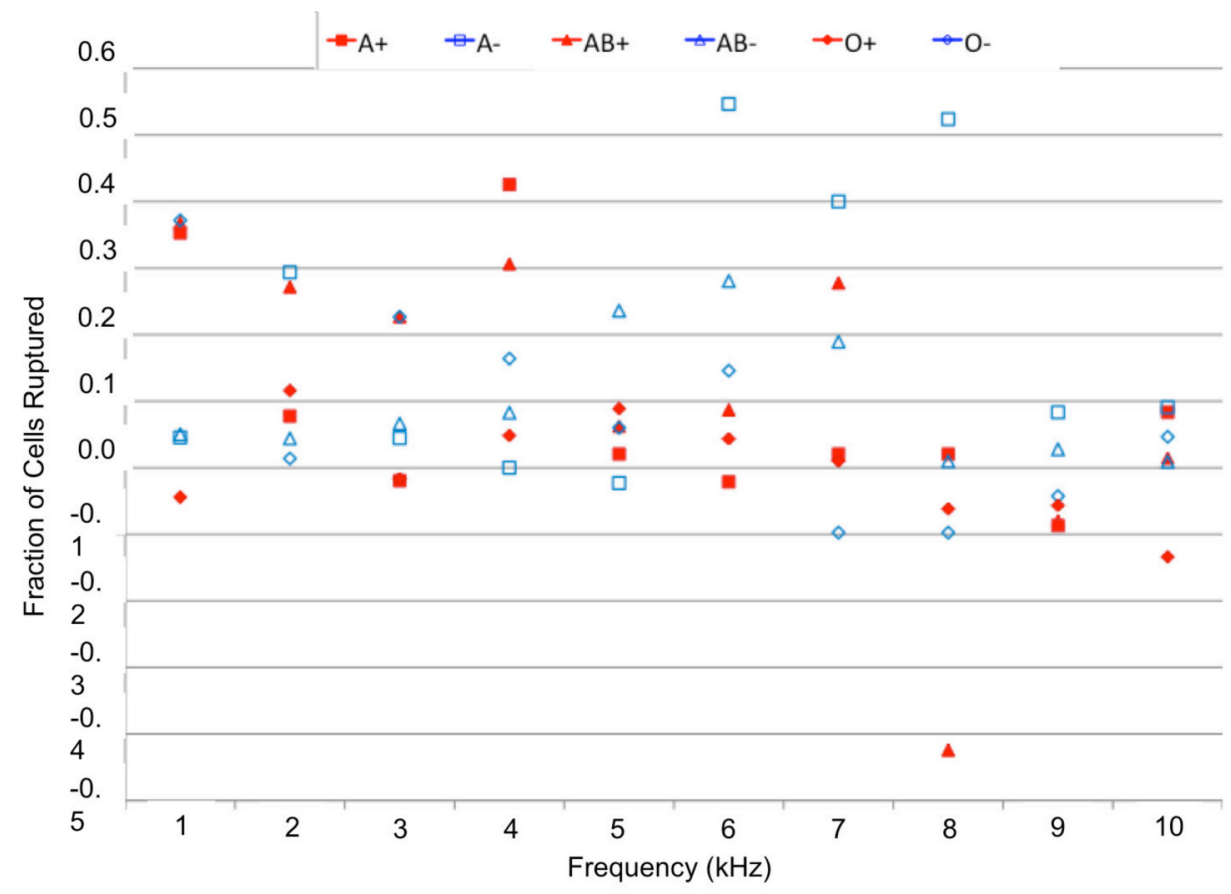

Figure 4.5. Final cell rupture fraction, $X_{f}$ versus applied frequency for $A+$ (red square), $A-$ (blue square), $A B+$ (red triangle), AB- (blue triangle), $\mathrm{O}+$ (red diamond) and $\mathrm{O}$ - (blue diamond). 
Erythrocyte rupture was explored in a dielectrophoretic field as a function of ABO-Rh blood type, donor, microdevice, field strength, washing, concentration, and frequency. It was found that for $\mathrm{A}+, \mathrm{A}-$ and $\mathrm{B}+$ a kinetic model could be fit to the time dependent rupturing behavior. Variation between donors, variation within a single donor across two donations and variation within a single donation across two microdevices were found to be insignificant. ANOVA analysis revealed a statistically significant aging dependency on O- erythrocyte rupture with regard to blood storage age, however even though the other blood types showed an increase in overall rupture fraction after Day 0 , none of this was statistically significant at $95 \%$ CI. There was no statistical significance found based on washing the cells prior to analysis. Two-factor ANOVA without replication showed that final rupture fraction increases with electrical field strength were significant.

\subsection{Conclusion}

Erythrocyte rupture was explored in a dielectrophoretic field as a function of ABO-Rh blood type, donor, microdevice, field strength, washing, concentration, and frequency. Results support the viability of using AC DEP fields for erythrocyte rupture and suggest differing rupturing kinetics of $\mathrm{ABO}-\mathrm{Rh}$ blood cells. A first order exponential decay model was fit to $\mathrm{A}+, \mathrm{A}-$ and $\mathrm{B}+$ time dependent rupture profiles. The other blood types, $\mathrm{AB}+, \mathrm{AB}-, \mathrm{O}+$ and $\mathrm{O}-$, did not experience significant rupturing and thus a kinetic model could not be fit to the data. The rupturing rate constant was significantly different

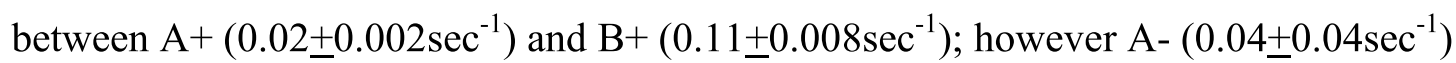
could not be distinguished based on a kinetic trend due to differing time-dependent behavior patterns between donors. As early as 5 minutes in a modest $(0.03 \mathrm{Vpp} /$ micron $)$ 
$\mathrm{DEP}$ field, it is possible to distinguish $\mathrm{B}+$ from $\mathrm{AB}-, \mathrm{O}+$ and $\mathrm{O}-$ at $95 \%$ confidence. In 10 minutes, $\mathrm{A}+$ and $\mathrm{A}-$ can be distinguished from $\mathrm{AB}-\mathrm{O}+$ and $\mathrm{O}-$ with $95 \%$ confidence. Final rupture fractions at 15 minutes had overlapping $95 \% \mathrm{CIs}$ between $\mathrm{A}+, \mathrm{A}-$ and $\mathrm{B}+$.

Final rupture fractions were also used to make comparisons and statistical analysis supported this as an alternative and simpler metric for ABO-Rh type comparisons. $\mathrm{B}+$ cells rupture more readily than any other blood type ending at $0.74 \pm 0.06$ rupture fraction. $\mathrm{A}+$ and $\mathrm{A}-$ also had a propensity to rupture at $0.29 \pm 0.11$ and $0.41 \pm 0.31$ final rupture fractions, respectively. Blood types $\mathrm{O}+, \mathrm{O}-$, and $\mathrm{AB}+$ do not experience appreciable rupturing, while AB- blood cells remained intact and experience positive DEP concentration up the electric field gradient at $1 \mathrm{kHz}$. Within the positive Rhesus blood group, the final rupture fraction increased from $\mathrm{AB}$ (none) to $\mathrm{O}$ to $\mathrm{A}$ to $\mathrm{B}$. A similar trend was observed for the negative blood types without B- data due to donor logistics. The presence of the Rhesus factor transmembrane protein lowered final rupture fractions for blood of type $\mathrm{O}, \mathrm{A}$ and $\mathrm{AB}$, suggesting possible differences in membrane integrity between negative and positive $\mathrm{Rh}$ of the same $\mathrm{ABO}$ antigen group. Thus, $\mathrm{ABO}$ and Rhesus factor antigens alter the cell's response to an electric stimulus, suggesting the Maxwell-Wagner membrane polarization phenomenon is dependent on exterior molecular membrane expression. Both the first order rupture kinetic model and the final rupture fractions suggest complex ion/ABO-Rh molecule/membrane DEP mediated interactions.

Variation between donors, variation within a single donor across two donations and variation within a single donation across two microdevices were found to be insignificant. Experiments revealed 0.002 to 0.282 variation within same donor tested 5 
days apart, and .0 .001 to 0.1 variation between microdevices with the same blood sample. The custom fabricated microdevices used in this research had larger tolerances than traditional clean room microfabricated devices. Blood type trends held between different microdevices, but confidence intervals and experimental errors would likely have been much smaller with micropatterned electrodes instead of wire. Concurrently, chemical and morphological changes to the device's platinum electrodes with field exposure ${ }^{225}$ could have influenced the dielectrophoretic behaviors observed.

Additional dependencies were explored to elucidate the best rupturing conditions as well as rupture mechanisms. Blood storage age effects were tested on the day of donation and every two days thereafter for six days. ANOVA analysis revealed a statistically significant aging dependency on O- erythrocyte rupture. Other blood types also showed slight increases in overall rupture fraction after Day 0 , but none of these changes were significant at $95 \%$ confidence.

A plethora of antibodies, proteins and biochemical indicator molecules exist in the blood plasma surrounding blood cells. To determine whether cell interactions with the plasma such as antigen-antibody interactions ${ }^{1}$ impacted dielectrophoretic rupture behaviors, cells were washed with isotonic phosphate buffer saline prior to $1 \mathrm{kHz}$ DEP rupture treatment. ANOVA analysis revealed no dependence on washing. Thus, washing would add an unnecessary and complicated step to a future microdevice. Further, plasma biomolecules or antigen-antibody interactions do not impact DEP membrane instabilities.

Dielectrophoretic forces on cells increase with increasing applied field strength, which was verified with increasing final rupture profiles from 0.015 to 0.03 to 0.06 
$\mathrm{V}_{\mathrm{pp}} / \mu \mathrm{m}$. Two-factor ANOVA without replication showed that final rupture fraction increases with electrical field strength were significant. Results suggest that rupture is in fact a DEP mediated effect and further increases in field strength will further increase rupture fractions.

To determine if cell/cell interactions influenced dielectrophoretic rupture behaviors, two blood types, $\mathrm{B}+$ and $\mathrm{O}-$, representing the highest and lowest rupture fractions were examined at four different volume-to-volume ratios. ANOVA analysis revealed no statistically significant differences in cell rupture with cell concentration. This result indicates that whole blood sample could be used on-chip and the fraction of cells ruptured over a given time period would stay consistent for a blood type regardless of the starting hematocrit level of the sample.

To determine if different blood types exhibited different optimal frequencies for cell rupture, frequency dependency studies between 1 and $10 \mathrm{kHz}$ were completed. A single donation each was tested for 50 minutes at frequencies ranging from $1-10 \mathrm{kHz}$ for $\mathrm{A}+, \mathrm{A}-, \mathrm{AB}+, \mathrm{AB}-, \mathrm{O}+$ and $\mathrm{O}-$. Statistical analysis couldn't be completed, but optimal frequencies varied by blood type further supporting the conclusion that $\mathrm{ABO}-\mathrm{Rh}$ expression influences DEP induced membrane instabilities and rupture. This result compliments the larger body of literature exploring dielectrophoretic behaviors of cells as a function of higher frequencies ${ }^{1,3-9,12-14,118,123,124,158,159,161,162,188-192}$. Thus frequency could potentially control the amount of rupture based on both exposure time and frequency for a given blood type.

This work has demonstrated the potential to rupture human erythrocytes using low frequency alternating current dielectrophoretic forces, potentially identify blood type 
by rupturing kinetics. DEP provides a low-cost, chemical-free and relatively safe way of rupturing cells that does not require highly technical fabrication techniques. Due to these factors, rupturing with AC DEP has advantages over chemical, mechanical and electroporation-induced lysis. This work has also shown that it is possible to rupture human erythrocytes, and that this rupture is dependent on the ABO-Rh blood type of the sample. This opens this technique up to possible uses ranging from subcellular analysis to blood type determination to therapeutic techniques. Further experiments are needed to tease out the exact kinetic rates of the other blood types and to look at medically significant confidence intervals of closer to $99 \%$.

\subsection{Acknowledgements}

The authors would like to acknowledge the undergraduate researchers who tested the different dependencies: Eric Rutan (Field Strength and Washing), Sean Duke (Concentration) and Philip Jamison (Frequency). All work was performed in an Institutional Biosafety Committee (IBC) certified Biosafety Level 2 laboratory and received approval from the Internal Review Board (IRB) at Mississippi State University for the protection of human subjects (Approval \#05-187). Work was supported by the NSF CBET CAREER (0633538/1041338) and NSF GRS (0737864, 0841403, 0942772). The authors have declared no conflict of interest. 



\section{Explorations of ABO-Rh Antigen Expressions on}

\section{Erythrocyte Dielectrophoresis: Changes in Cross-over}

\section{Frequency $^{1}$ *}

\subsection{Abstract}

A quadrupole dielectrophoretic microdevice was utilized to examine the $\mathrm{ABO}-\mathrm{Rh}$ dependencies on erythrocyte polarizations. This important step toward medical microdevice technology would transform key clinical blood tests from the laboratory into the field. Previous work in dielectrophoretic microdevices demonstrated that the large number of $\mathrm{ABO}$ antigens on erythrocyte membranes impacts their dielectrophoretic signature at $1 \mathrm{MHz}$. This work explores the dielectrophoretic behavior of native human erythrocytes categorized by their ABO-Rh blood types and directly compares these responses to the same erythrocyte sample modified to remove the A and B antigens. A $\beta(1-3)$-galactosidase enzyme was utilized to cleave the $\mathrm{ABO}$ polysaccharide backbone at the galactosidase bonds. The enzymatic reaction was optimized by comparing agglutination of the native and modified blood cells in addition to UV-Vis and HPLC analysis of the reaction effluent for saccharide residues. Next, the dielectrophoretic behaviors of the native and modified erythrocytes were visually verified in a quadrupole electrode microdevice over a frequency range from $100 \mathrm{kHz}$ to $80 \mathrm{MHz}$. The lower cross-over frequency (COF), which transitions from negative to positive dielectrophoresis,

\footnotetext{
${ }^{*}$ The material contained in this chapter was previously published in the journal Electrophoresis
} 
for $\mathrm{ABO}$ blood types tested ( $\mathrm{A}+, \mathrm{A}-, \mathrm{B}+, \mathrm{B}-, \mathrm{AB}+, \mathrm{O}+$ and $\mathrm{O}-)$ differed over the range from $17-47 \mathrm{MHz}$. The cross-over frequencies of the corresponding enzyme modified erythrocytes were also determined and the range narrowed to $29-41 \mathrm{MHz}$. A second crossover frequency in the $70-80 \mathrm{MHz}$ range was observed and was reduced in the presence of the transmembrane Rhesus factor. These results suggest that antigen expression on erythrocyte membrane surfaces influence cell polarizations in nonuniform AC fields.

\subsection{Introduction}

Dielectrophoresis has been shown to distinguish native and altered cells based on infection, live / dead status, and treatments with membrane alterations 3,5,162,164,226,227. Cells infected with malaria were separated from healthy cells due to changes induced in the host cell by the parasite. An additional cytosol and membrane develop within the host cell changing the permittivity and conductivity thus leading to a change in ClausiusMossotti factor and dielectrophoretic force ${ }^{5}$. Healthy blood cells were shown to have a different cross-over frequency (COF) than HL-60 cells and T-lymphocytes as well as different electrorotation spectra ${ }^{3}$. It has also been seen that erythrocytes infected with Babesia bovis virus have significantly different dielectrophoretic responses than cells without viral infection ${ }^{226}$. Live versus dead yeast cells also exhibit different cross-over frequencies especially as medium conductivity increases. Non-viable yeast cells were observed to have a much higher low cross-over frequency and a much lower high crossover frequency than viable cells ${ }^{162}$. Lastly, electroporated cells were separated from non-electroporated cells at a medium conductivity of $0.174 \mathrm{~S} / \mathrm{m}$ and a frequency of $2 \mathrm{MHz}$ 227 
Our group has focused on the dielectrophoretic responses of erythrocytes categorized by their $\mathrm{ABO}-\mathrm{Rh}$ antigens. Erythrocytes are a unique system because both surface $(\mathrm{ABO})$ and transmembrane $(\mathrm{Rh})$ antigens are expressed in combinations with each other; further the cells demonstrate genetic diversity via a plethora of other antigens. The ABO-Rh antigen's influence on dielectrophoretic polarizability is more complex than the present / not present differences demonstrated in references 1 through 6 . Prior results in a perpendicular electrode configuration at $1 \mathrm{MHz}$ revealed that $\mathrm{O}+$ could be distinguished from $\mathrm{A}+, \mathrm{B}+$, and $\mathrm{AB}+$ with greater than $95 \%$ confidence ${ }^{11}$. In a $\mathrm{DC}$ insulator dielectrophoretic device, $\mathrm{A}+$ was distinguishable from all 7 other $\mathrm{ABO}-\mathrm{Rh}$ blood types at $99.986 \%$ confidence $^{10}$. This work demonstrates that treatment of erythrocytes with $\beta(1-3)$ galactosidase successfully cleaves the $\mathrm{ABO}$ polysaccharide antigens from the membrane surface such that they no longer react with their corresponding antibodies (agglutination). This work further demonstrates that this modification changes the dielectric properties of the erythrocyte and alters the cross-over frequency observed. Results were also compiled for the presence/absence of the Rhesus factor. Combined, this demonstrates the influence of $\mathrm{ABO}-\mathrm{Rh}$ antigens on an erythrocyte's dielectrophoretic response.

Human erythrocytes are biconcave disks of major radius $3-4 \mu \mathrm{m}$ and minor radius 0.25 to $1 \mu \mathrm{m}^{26}$. Their unusual shape allows them to maximize surface area with respect to volume for efficient oxygen and carbon dioxide exchange throughout the human body. There are currently 30 blood typing systems recognized by the International Society for Blood Transfusions; the most prominent is the $\mathrm{ABO}-\mathrm{Rh}$ combination due to its essential role in determining blood transfusion compatibility ${ }^{127}$. The ABO system consists of 
three antigens (A, B and $\mathrm{O}$ ) which are surface polysaccharides that differ from each other by a terminal sugar ${ }^{119}$. All three antigens have a backbone of bonded phosphingolipid to galactose to $\mathrm{N}$-acetylglucosamine to a second galactose to a fucose. For the A antigen, the terminal galactose is also bonded to an $\mathrm{N}$-acetylgalactosamine, while for the $\mathrm{B}$ antigen, the terminal galactose is also bonded to a third galactose. The Rhesus factor is a group of transmembrane antigens that are hypothesized to function as ammonium transporters (AMT) due to the homology similarities between the human RHAG protein and the animal erythrocyte equivalent of AMT ${ }^{143}$ Blood is classified as positive if it expresses RHAG and negative if it does not ${ }^{120,121}$.

Dielectrophoresis, the movement of polarizable particles in heterogeneous electric fields, has expanded considerably since Pohl's initial explorations into the AC polarization of neutral matter ${ }^{15}$. The dielectrophoretic force equation is dependent on the effective dipole vector, $\vec{m}$, and the gradient of the squared electric field $\nabla \vec{E}^{2}$ :

$$
\vec{F}_{D E P}=(\vec{m} \cdot \nabla) \vec{E}^{2}
$$

The effective dipole vector is given by:

$$
\vec{m}=3 V \varepsilon_{m} f_{C M} \vec{E}(\omega)
$$

Where $V$ is the volume of the particle of interest, $\varepsilon_{m}$ is the polarizability of the medium and $f_{C M}$ is the Clausius-Mossotti factor. The Clausius-Mossotti factor is comprised of the polarizability of the particle and medium $\left(\varepsilon_{p}\right.$ and $\left.\varepsilon_{m}\right)$ as well as the conductivity of the particle and the medium $\left(\sigma_{p}\right.$ and $\left.\sigma_{m}\right)$ in the form of the complex permittivity of the particle $\left(\varepsilon_{p}{ }^{*}\right)$ and the medium $\left(\varepsilon_{m}^{*}\right)$. Where the complex permittivity is a frequency $(\omega)$ dependent combination of the permittivity and conductivity:

$$
\varepsilon^{*}=\varepsilon+j \sigma / \omega
$$


The $f_{C M}$ equation has been expanded to separately represent the cytosol and membrane of a particle (in this case erythrocyte) in an ellipsoidal core-shell configuration as follows.

$$
f_{C M, r}=\frac{\frac{\frac{\varepsilon}{\text { membrane }}_{\text {cytosol }}^{*}}{\frac{d}{r} \varepsilon_{\text {cytosol }}^{*}+\varepsilon_{\text {membrane }}^{*}}-\varepsilon_{m}^{*}}{3\left[\varepsilon_{m}^{*}+L_{r}\left(\frac{\varepsilon_{\text {membrane }}^{*} \varepsilon_{\text {cytosol }}^{*}}{\frac{d}{r} \varepsilon_{\text {cytosol }}^{*}+\varepsilon_{\text {membrane }}^{*}}-\varepsilon_{m}^{*}\right)\right]}
$$

Where $r$ represents either $a$ for long axis radius or $c$ for the short axis radius of the cell, $d$ is the membrane thickness and $L_{r}$ is the depolarization factor, which is given by equation (5) ${ }^{115}$ :

$$
L_{r}=\frac{a^{2} c}{2} \int_{0}^{\infty} \frac{1}{\left(l+r^{2}\right) \sqrt{\left(l+a^{2}\right)^{2}\left(l+c^{2}\right)}} d l
$$

The cytosol and membrane complex permittivities, $\varepsilon_{\text {cytosol, and }}^{*}{ }_{\text {membrane }}{ }^{228}$, respectively follow equation (3) and therefore depend on the permittivity $\left(\varepsilon_{c y t o s o l}\right)$ and conductivity $\left(\sigma_{\text {cytosol }}\right)$ of the cytosol and the permittivity $\left(\varepsilon_{\text {membrane }}\right)$ and conductivity $\left(\sigma_{\text {membrane }}\right)$ of the membrane. The frequency in equation (3) and subsequently in equation (4) at which the real part of the Clausius-Mossotti factor is equal to zero is known as the cross-over frequency, which is dependent on the permittivity and conductivity of the membrane, the cytosol and the medium ${ }^{12,112,164}$. Based on the cell's intrinsic frequency-dependent polarizability as captured by $f_{C M}, \operatorname{Re}\left(f_{C M}\right)$ can be positive such that the cell will experience positive dielectrophoresis and move towards regions of high squared electric field gradients or $\operatorname{Re}\left(f_{C M}\right)$ can be negative and the cell will experience negative dielectrophoresis and move towards regions of low squared electric field gradients ${ }^{15}$. In the case of human erythrocytes, which lack nuclei, this polarizability is based on the 
permittivity and conductivity of the cytosol and the permittivity and conductivity of the membrane as described in equation (4). Antigens are expressed on or within the cell membrane such that the permittivity and conductivity of the cell membrane change thus altering the sign of the dielectrophoretic response and the $\mathrm{COF}$, which was observable in our quadrupole microdevice for each of the blood types tested.

This paper describes the successful enzymatic removal of $\mathrm{ABO}$ antigens from erythrocyte membranes and then explores the dielectrophoretic cross-over frequency of the native human erythrocytes of known $\mathrm{ABO}-\mathrm{Rh}$ blood type $(\mathrm{B}+, \mathrm{B}-, \mathrm{A}+, \mathrm{A}-, \mathrm{AB}+, \mathrm{O}+$ and O-) and compares with the same erythrocyte sample after the enzyme modification. The ABO backbone contains two galactose molecules, so $\beta 1-3$ galactosidase was selected as a specific exoglycosidase to catalyze hydrolysis reactions of both $\beta 1-3$ and $\beta 1-6$ (to a lesser degree) galactose ${ }^{229,230}$. This enzyme is traditionally utilized in the production of galacto-oligosaccharides to improve food quality and stimulate bacteria growth ${ }^{230}$. To remove the $\mathrm{ABO}$ antigens, erythrocytes were incubated with $\beta 1-3$ galactosidase for half an hour; the erythrocytes were then dielectrophoretically examined to determine if these polysaccharide antigens were responsible for the difference in COF seen with the native blood types. Three comparisons were made: 1) comparison between the native (unmodified) erythrocytes and between the enzyme modified erythrocytes, 2) comparisons between erythrocytes of the same $\mathrm{ABO}$ expression, but differing $\mathrm{Rh}$ expressions, and 3) comparison between the enzyme modified erythrocytes and the $\mathrm{O}$ type with the same Rh expression. 


\subsection{Materials and Methods}

\subsubsection{Materials}

Human blood samples of types $\mathrm{B}+, \mathrm{B}-, \mathrm{A}+, \mathrm{A}-, \mathrm{AB}+, \mathrm{O}+$ and $\mathrm{O}-$ were obtained from voluntary donors of a verified blood type via venipuncture by a trained phlebotomist at the Portage Health Clinic at Michigan Technological University. Blood was stored at $4^{\circ} \mathrm{C}$ and tested on the day of donation to avoid aging effects because phospholipids composition in the membrane changes with storage ${ }^{231}$ and that $\mathrm{pH}$, hemolysis, $\mathrm{K}+$ concentration and erythrocyte morphology alters during storage ${ }^{232}$. Erythrocytes storage has also been shown to reduce erythrocyte polarizability and impact DEP behavior ${ }^{23}$. The suspending medium was a $4 \%$ dextrose solution comprised of $6 \mathrm{~g}$ dextrose, $0.4 \mathrm{~g} \mathrm{NaCl}, 0.5 \mathrm{~g} \mathrm{KH}_{2} \mathrm{PO}_{4}$ and $0.24 \mathrm{~g} \mathrm{~K}_{2} \mathrm{HPO}_{4}$ in $150 \mathrm{~mL}$ E-pure water. $\mathrm{pH}$ was adjusted to 7.0 with $1 \mathrm{M} \mathrm{NaOH}$ or $2 \mathrm{M} \mathrm{HCl}$. The final solution conductivity was $0.9 \mathrm{~S} / \mathrm{m}$ and is on the order of physiological conditions of pure plasma. An equation describing the conductivity of stationary blood ${ }^{133}$ shows increases as the hematocrit level (fraction of cells) decreases until the pure plasma limit of $1.57 \mathrm{~S} / \mathrm{m}$ is reached in Table 5.1. Pooled plasma from donors in this study was measured as $1.1 \mathrm{~S} / \mathrm{m}$. Traditional DEP cross-over frequency analysis has been done at conductivities below $0.1 \mathrm{~S} / \mathrm{m}$ $3,4,8,140,162,164,167,226,227,233$. However, operating at physiological conductivities is diagnostically advantageous and facilitated a hybrid electrokinetic technique at $\sim 1 \mathrm{~S} / \mathrm{m}^{234}$. 
Table 5.1.

Conductivity of whole blood (S/m) based on calculations from Vissers ${ }^{133}$. Pure plasma shown to have conductivity of $1.57 \mathrm{~S} / \mathrm{m}$.

\begin{tabular}{|c|c|c|c|}
\hline $\begin{array}{c}\text { Hematocrit } \\
\text { Value }\end{array}$ & Meaning & C number & $\begin{array}{c}\text { Blood } \\
\text { Conductivity }(\mathrm{S} / \mathrm{m})\end{array}$ \\
\hline 0 & No erythrocytes & 1.91 & 1.57 \\
\hline & $\begin{array}{c}\text { Anemic Threshold } \\
\text { Women }\end{array}$ & 1.91 & 0.81 \\
\hline 0.33 & Low Normal Women & 1.91 & 0.74 \\
\hline 0.37 & Anemic Threshold Men & 1.91 & 0.71 \\
\hline 0.39 & Low Normal Men & 1.91 & 0.61 \\
\hline 0.45 & High Normal Women & 1.91 & 0.57 \\
\hline 0.48 & High Normal Men & 1.91 & 0.51 \\
\hline 0.52 & Polycythemic Threshold & 1.91 & 0.47 \\
\hline 0.55 & Extreme Polycythemia & 1.91 & 0.18 \\
\hline 0.8 & \multicolumn{2}{|l}{} \\
\hline
\end{tabular}

\subsubsection{Microdevice Fabrication}

Microdevices were constructed with micropatterned electrodes and PDMS soft photolithography. To create gold plated electrodes, positive photoresist (Futurrex PR1000A, Franklin, NJ) was spun onto glass slides (Laurell Technologies, North Wales, PA) and UV exposed (EVG620, EVG, AZ) via soft photolithography ${ }^{33,223,224}$. Undesired features were removed with isopropanol. The slides were then sputter coated with a thin adhesive chrome layer followed by gold to a depth of 200nm (Perkin-Elmer Randex Sputtering System Model 2400, Perkin-Elmer, USA). Excess photoresist and sputter metals were removed from the glass slide via sonication with acetone (VWR), leaving the gold electrodes pattern, which consists of $100 \mu \mathrm{m}$ electrodes spaced $50 \mu \mathrm{m}$ apart. This slide was later adhered to the PDMS casting of the device.

For the PDMS fluidic layer, positive SU-8 2025 (Microchem, MA, USA) was spun onto 4 inch Si wafers (WRS Materials, CA) to $\sim 70 \mu \mathrm{m}$ and pattered via UV exposure (EVG 620, EVG, AZ) at 20W for 10 seconds. PDMS castings were made of the 
patterned wafer with a 10:1 PDMS elastomer and curing agent at $60^{\circ} \mathrm{C}$ for 3 hours (Isotemp Model 280A, Fisher Scientific, Pittsburgh, PA). The casting was removed, then cut to size and ports punched for electrode connections and sample injection/removal. The casting and electrode patterned glass slide were subjected to oxygen plasma (Harrick Plasma PDC-001, Harrick Plasma, Ithaca, NY), then a microscope was used to align the casting/electrodes as shown in Figure 5.1 before pressure and heat bonding. Sealing was verified with dye. Copper wires were connected to the gold plated electrodes using silver epoxy (ITW Chemtronics, Kennesaw, GA) hardened in the oven at $90^{\circ} \mathrm{C}$.

\subsubsection{Antigen Modification}

To directly quantify the contribution of $\mathrm{ABO}$ antigens to the dielectrophoretic behavior independent of other antigen expression, blood cells were chemically altered to remove $\mathrm{A}, \mathrm{B}$, and $\mathrm{O}$ antigens from the membrane. $\beta(1-3)$ galactosidase (New England Biolabs, Ipswich, MA) was selected to break the $1 \rightarrow 3$ linkage of galactose in the antigen backbone. The enzymatic reactants were (Fig. 1): $66.7 \%$ packed erythrocytes, $0.1 \mathrm{U} / \mu \mathrm{L}$ $\beta(1-3)$ galactosidase, $10 \%$ G2 reaction buffer (New England Biolabs, Ipswich, MA), 1\% bovine serum albumin (New England Biolabs, Ipswich, MA) and 21.3\% $\mathrm{NaCl}$ (VWR) (volume to total volume percentages). Packed erythrocytes were obtained by centrifuging a whole blood sample at $1400 \mathrm{rpm}$ for 5 minutes, removing the plasma and then washing the cells twice with isotonic $0.9 \mathrm{wt} \% \mathrm{NaCl}$. The enzymatic reaction mixture was oven incubated at $40^{\circ} \mathrm{C}$ for 30 minutes. The reaction was stopped with the addition of $4 \mathrm{~mL}$ of $\mathrm{NaCl}$ and centrifugation to separate the effluent from the modified erythrocytes. 
The effluent was tested for sugars using UV-Vis (Genesys 10, ThermoSpectronic, Waltham, MA) and HPLC. The carbohydrate HPLC column using ion-moderated partition chromatography, had a flow rate of $0.6 \mathrm{~mL} / \mathrm{min}$ with a mobile phase of distilled and deionized water. Sugar residues were identified and compared to both literature values and standards run on the same HPLC column (Aminex HPX-87P, Biorad, CA). Standards were galactose, fucose (VWR, PA), and an A-trisaccharide (Accurate Chemical, NY) which has the same polysaccharide structure as the A antigen. The native and modified blood cells were both tested for agglutination with Anti-A and Anti-B serum (Edvotex, Bethesda, MD). One drop of Anti-A serum was added to one native and one modified blood well; similarly with Anti-B serum. The respective mixtures were then placed between a glass slide and a cover slip and imaged using the LabSmith microscope (LabSmith, CA). Bulk responses and 25X microscope images were recorded. Prior to COF experiments, zeta potentials of the native and modified erythrocyte suspensions at DEP experiment dilutions were measured using a NanoSeries ZetaSizer (Malvern, Worcestershire, UK). Five measurements of each were performed to determine an average and a 95\% CI. 


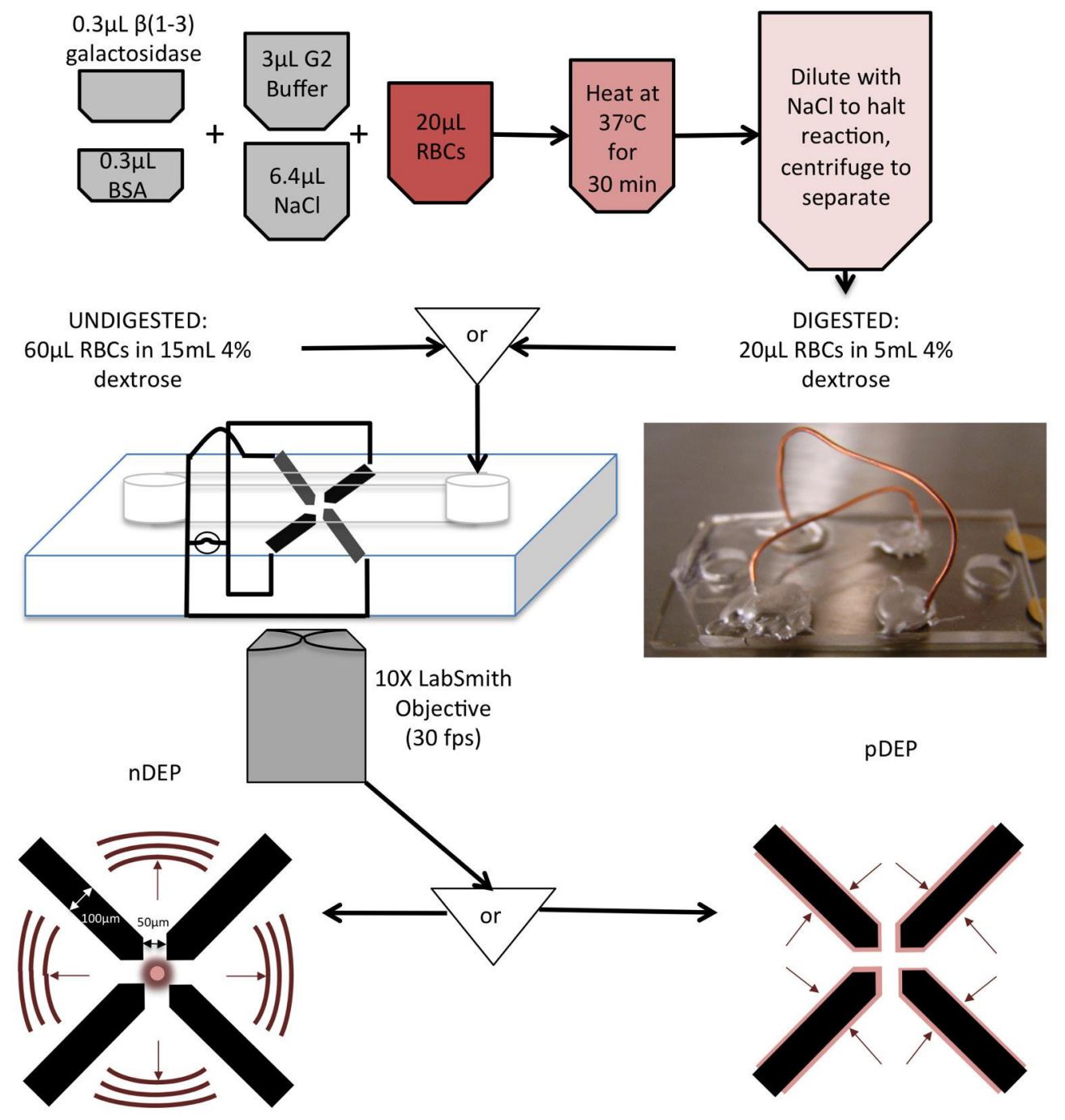

Figure 5.1. Enzymatic reaction recipe and procedure. Dilution conditions are given for both native and modified blood, which are separately loaded into the microdevice. The gold-plated quadrapole electrodes (width of $100 \mathrm{~mm}$ and spacing of $50 \mathrm{~mm}$ ) in the PDMS-glass microdevice are connected to an AC generator and erythrocyte frequency responses are recorded with 10x and20x LabSmith video microscopy. Responses are categorized based on the positive and negative dielec- trophoresis patterns shown.

\subsubsection{Dielectrophoresis Experiments}

Two erythrocyte dilutions were prepared for each blood donation. The first

solution was native blood, centrifuged at 1400rpm for 5 minutes and washed two times in $0.9 \% \mathrm{NaCl}$ solution to recover packed blood cells for $1: 250 \mathrm{~V}: \mathrm{V}$ resuspension in $0.9 \mathrm{~S} / \mathrm{m}$ dextrose solution. Post-enzyme modification, the packed blood cells were dilution at a 
1:125 ratio gave a comparable cell density in the device. Between experiments, the device was washed with E-pure water and cleanliness verified optically.

Alternating current dielectrophoresis experiments were performed on quadrupole electrode configurations using an $\mathrm{AC}$ waveform generator (Agilent 33250A, Agilent, Santa Clara, CA). The electric field density was fixed at $0.1 \mathrm{~V}_{\mathrm{pp}} / \mu \mathrm{m}$ and the frequency was adjusted. 10X videos at $30 \mathrm{fps}$ were taken using a LabSmith SVM Synchronized Video Microscope (LabSmith, CA) for 15 seconds at the following frequencies: $100 \mathrm{kHz}$, $500 \mathrm{kHz}, 1 \mathrm{MHz}, 10 \mathrm{MHz}, 20 \mathrm{MHz}, 30 \mathrm{MHz}, 40 \mathrm{MHz}, 50 \mathrm{MHz}, 60 \mathrm{MHz}, 70 \mathrm{MHz}$ and $80 \mathrm{MHz}$ (generator maximum). Once initial COF ranges were identified, closely-spaced frequencies were tested to determine a more exact COF.

\subsection{Results}

\subsubsection{Antigen Modification}

Three different analytical methods were used to verify successful modification in the post- $\beta(1-3)$ galactosidase modified blood samples: UV-Vis spectrophotometry, HPLC, and basic agglutination tests. Reaction conditions were systematically explored

to determine the optimal procedure to a) avoid cell lysis, b) successfully cleave galactose (determined by detection of polysaccharide residues in the supernatant), and c) achieve null agglutination against the blood type's corresponding antibody.

\subsubsection{UV-Vis Results}

Absorbance was measured for each enzymatically modified sample effluent as well as polysaccharide controls from $190 \mathrm{~nm}$ to $1100 \mathrm{~nm}$. In Figure $5.2 \mathrm{~A}$, five peaks at 
$\sim 280,340,400,530$ and $580 \mathrm{~nm}$ were evident. The broad peak at 400nm corresponds to

literature values for heme ${ }^{235,236}$. Large peak absorbance, such as that observed with the 1 hour $\mathrm{A}+0.2 \mathrm{U} / \mu \mathrm{L} \beta(1-3)$ galactosidase reaction run, indicated the enzyme had caused erythrocyte rupture. The $1 \mathrm{hr} \mathrm{A}+0.05 \mathrm{U} / \mu \mathrm{L} \beta(1-3)$ galactosidase reaction showed a small heme peak, but the blood still reacted with the Anti-A serum in the agglutination tests indicating sufficient antigen cleavage was not achieved. Optimal reaction conditions were 0.5 hour at $40^{\circ} \mathrm{C}$ at $0.1 \mathrm{U} / \mu \mathrm{L}$. A peak at $280 \mathrm{~nm}$ increased with increasing reaction concentration/time and was attributed to cytosol proteins. Two peaks at 530 and $580 \mathrm{~nm}$ were attributed to non-enzyme derived blood cell components because the same peaks show up in native erythrocyte solutions pre and post vortex rupturing. Figure 5.2B demonstrates that individual enzymatic reactants (BSA, Beta-galactosidase, $\mathrm{NaCl}$ and $\mathrm{G} 2$ reaction buffer) have negligible absorbance in the 300 to $900 \mathrm{~nm}$ range suggesting that the post digestion peaks at $280 \mathrm{~nm}$ and $340 \mathrm{~nm}$ were likely polysaccharide residues.

\subsubsection{HPLC Results}

Figure 5.2C shows a standard galactose peak while elution times and peak heights were tabulated in Table 5.2 including four standards (fucose, galactose, $\beta(1-3)$ galactosidase and A-trisaccharide) and four trial erythrocyte modification effluents (A+ $0.2 \mathrm{U} / \mu \mathrm{L} \beta(1-3)$ galactosidase $1 \mathrm{hr}, \mathrm{A}+0.05 \mathrm{U} / \mu \mathrm{L} \beta(1-3)$ galactosidase $1 \mathrm{hr}, \mathrm{A}+0.1 \mathrm{U} / \mu \mathrm{L}$ $\beta(1-3)$ galactosidase $1 / 2 \mathrm{hr}, \mathrm{B}-0.1 \mathrm{U} / \mu \mathrm{L} \beta(1-3)$ galactosidase $1 / 2 \mathrm{hr}$ ) and correspond to the same samples in Figure 5.2A\&B. A $\beta(1-3)$ galactosidase peak elutes at 6.7 minutes in the standard solution and was evident in all four erythrocyte-antigen modification 
effluents at heights consistent with extents of reaction. The 6.7 min peak was also present in the fucose and galactose standards and was attributed to the similar sugar configuration and ion affinity to $\beta(1-3)$ galactosidase. The peak at 12.5 minutes for the $0.05 \mathrm{U} / \mu \mathrm{L} \beta(1-3)$ galactosidase run is likely due to the incomplete digestion of the sugar residues. The primary peak for A-trisaccharide was at 28.4 minutes with a secondary peak at 10.5 minutes. Peaks were observed at 28.4 minutes for the $0.05 \mathrm{U} / \mu \mathrm{L}$ and $0.1 \mathrm{U} / \mu \mathrm{L}$ $\mathrm{A}+$ digestion runs, but not for the $0.2 \mathrm{U} / \mu \mathrm{L} \mathrm{A}+$ or $0.1 \mathrm{U} / \mu \mathrm{L}$ B- digestions suggesting that the A trisaccharide residues disappeared with increasing extents of reaction. The absence of the 28.4 minute peak for the B- blood was as expected. The fucose standard eluted at 16.5 minutes with minor peaks at 12.5 and 15.2 minutes. Also at 15.2 minutes was the primary galactose peak with minor peaks at 12.5 and 28.4 minutes. These results suggest similar affinity chemistry and fragments between the sugar standards and support UV-vis conclusions on the extent of reaction at difference concentrations. 

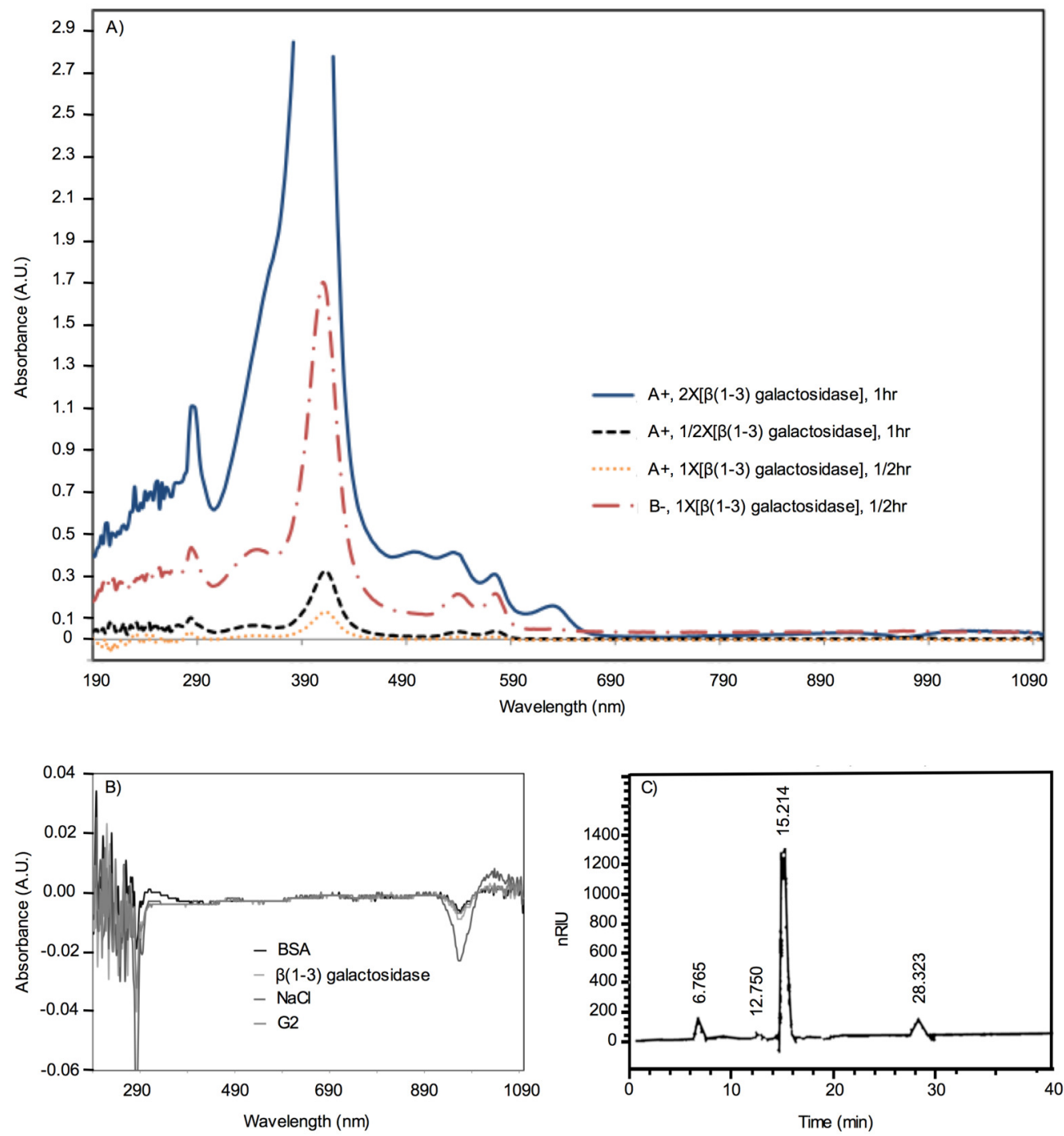

Figure 5.2. (A) UV-Vis analysis of effluent from red blood cells modified with b(1-3)-galactosidase under four different reaction conditions. (B) UV-Vis traces of the enzyme reaction components (BSA, b-galactosidase, $\mathrm{NaCl}$, G2) demonstrating that these do not contribute to peaks from 300 to $900 \mathrm{~nm}$. (C) Representative HPLC trace for galactose standard. 
Table 5.2.

HPLC elution times and peak heights for four standards (fucose, galactose, (1-3) galactosidase and Atrisaccharide) and four trail erythrocyte modification effluents $(A+0.2 U / \mu L \beta(1-3)$ galactosidase $1 \mathrm{hr}, A+1 / 2 X$ $\beta(1-3)$ galactosidase $1 \mathrm{hr}, \mathrm{A}+0.1 \mathrm{U} / \mu \mathrm{L} \beta(1-3)$ galactosidase $1 / 2 \mathrm{hr}$, and $\mathrm{B}-\mathrm{0.1 \textrm {U }} / \mu \mathrm{L} \beta(1-3)$ galactosidase $1 / 2 \mathrm{hr}$.)

\begin{tabular}{|c|c|c|c|c|c|c|}
\hline & \multicolumn{6}{|c|}{ Elution Time } \\
\hline Specimen & 6.7 & 10.5 & 12.5 & 15.2 & 16.5 & 28.4 \\
\hline Fucose & 156 & & 90.1 & 378 & 65002 & \\
\hline Galactose & 155 & & 39.6 & 1710 & & 135 \\
\hline$\beta(1-3)$ galactosidase & 156 & & 37.6 & & & \\
\hline A-trisaccharide & & 47.0 & & & & 219 \\
\hline $\begin{array}{l}A+0.2 U / \mu L \beta(1-3) \text { galactosidase } \\
1 \mathrm{hr}\end{array}$ & 128 & & & & & \\
\hline $\begin{array}{l}A+0.05 U / \mu L \beta(1-3) \text { galactosidase } \\
1 \mathrm{hr}\end{array}$ & 143 & & 33.2 & & & 91.2 \\
\hline $\begin{array}{l}B-0.1 U / \mu L \beta(1-3) \text { galactosidase } \\
1 / 2 \mathrm{hr}\end{array}$ & 133 & & & & & \\
\hline $\begin{array}{l}\text { A+ } 0.1 U / \mu L \beta(1-3) \text { galactosidase } \\
1 / 2 \mathrm{hr}\end{array}$ & 126 & & & & & 141 \\
\hline
\end{tabular}

\subsubsection{Agglutination Results}

Upon completion of the enzymatic digestion, the erythrocytes were tested against the Edvotex agglutination kit as described previously. Anti-B serum yields an agglutination reaction with the $\mathrm{B}$ antigen while anti-A serum yields an agglutination reaction with the A antigen. Figure 5.3A\&D are control images of B- and $0.1 \mathrm{U} / \mu \mathrm{L} \beta(1-$ 3) galactosidase modified B- erythrocyte suspensions, respectively at $25 \mathrm{X}$ without either antibody present. Figure 5.3B\&E show these samples contacted with anti-A serum and agglutination was not observed. Figure 5.3C\&F show the samples contacted with anti-B serum. Substantial agglutination was observed for the unmodified B- blood while slight erythrocyte swelling was observed in the modified erythrocyte/anti-B serum likely due to a mismatch in isotonicity. However, antigen/antibody binding events linking cells together is not any greater than is displayed with the sample in the anti-A serum 
suggesting successful cleavage of the B antigens. Similar results were observed for the other blood types (B+, $\mathrm{A}+, \mathrm{A}-, \mathrm{AB}+, \mathrm{O}+$ and $\mathrm{O}-)$.
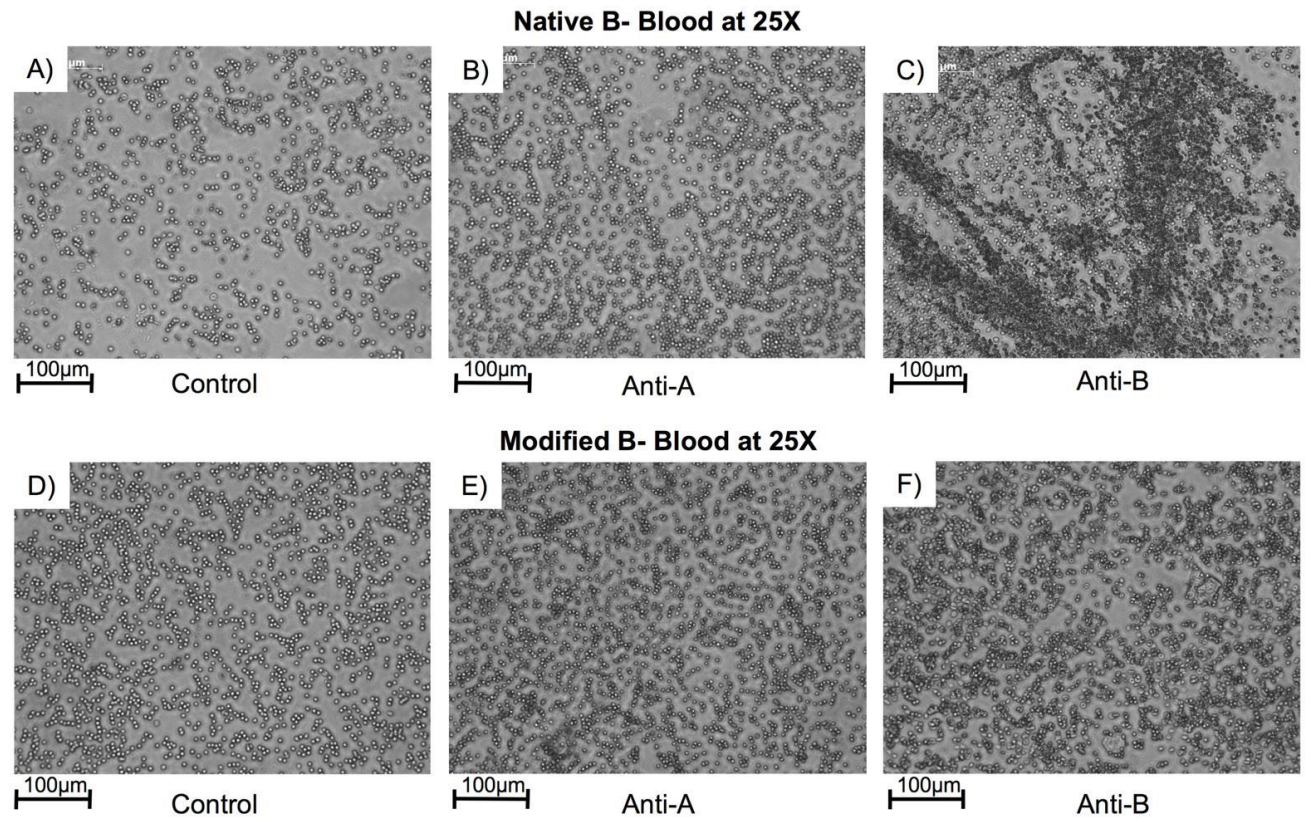

Figure 5.3. Microscope images of native and modified B- blood agglutination results at 25x. Native B- blood (A) without serum, (B) with anti-A serum and (C) with anti-B serum. $\beta(1,3)$-Galactosidase-modified B- blood (D) without serum, (E) with anti-A serum and (F) with anti-B serum. (C) shows B- agglutination with a substantially attenuated cell agglutination is observed in (F). The enzyme damages membrane integrity so intact cell counts drops upon digestion.

\subsubsection{Zeta potential measurements}

Prior to COF experiments, the native and modified erythrocyte zeta potentials were measured. At $95 \%$ confidence, the native suspension $(-15.86 \mathrm{mV})$ was the same as the modified suspension $(-15.92 \mathrm{mV})$. This suggests that cleavage of the sugars did not appreciably alter the membrane surface charge, which makes sense because the base of the antigen backbone (phosphingolipid to galactose to $\mathrm{N}$-acetylglucosamine) is likely intact after enzyme digestion and would therefore express a similarity of charge with the unmodified erythrocyte antigen. 


\subsubsection{Cross-over Frequency (COF)}

Native erythrocyte suspensions and the corresponding modified erythrocyte suspensions of a single donation from each of the blood types $(\mathrm{B}-, \mathrm{B}+, \mathrm{A}+, \mathrm{A}-, \mathrm{AB}+, \mathrm{O}+$ and $\mathrm{O}-$ ) were tested in a quadrupole microdevice at $0.1 \mathrm{Vpp} / \mu \mathrm{m}$ and at frequencies ranging from $100 \mathrm{kHz}$ to $80 \mathrm{MHz}$. Once transition ranges were determined for the lower and higher COFs, DEP responses at $1 \mathrm{MHz}$ intervals were performed. All blood types experienced strong negative dielectrophoresis at $100 \mathrm{kHz}$ judged by rapid movement to the center of the four electrodes (Figure 5.1). This is evident by comparing time zero (no field) to 2 seconds in Figure 5.4A (B- native) and Figure 5.4D (B- enzyme modified). For the B- in Figure 5.4B (42MHz), the cells experienced both pDEP and nDEP, but for the modified B- in Figure 5.4E, the cells are extremely close to their COF and demonstrate very weak positive dielectrophoresis and very weak negative dielectrophoresis. Both the native and modified B- cells again display nDEP at $80 \mathrm{MHz}$ (Figure 5.4C\&F). 
Native B- Blood at 20X

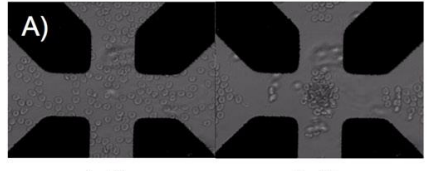

$\mathrm{t}=0 \mathrm{~s}$ $100 \mathrm{kHz}$

$t=2 s$

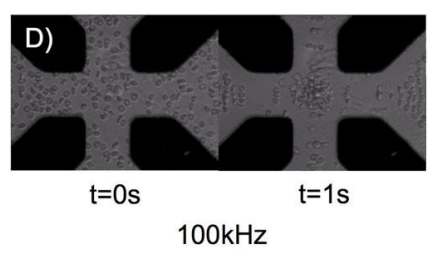

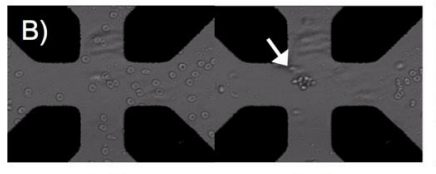

$\mathrm{t}=0 \mathrm{~s}$ $42 \mathrm{MHz}$

Modified B- Blood at 20X

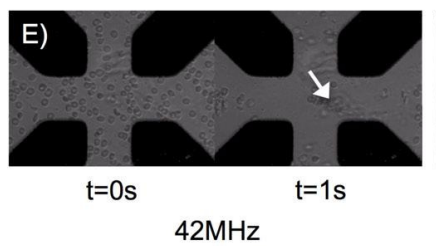

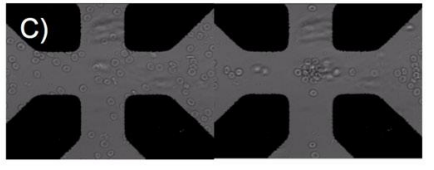

$t=0 s$ $80 \mathrm{MHz}$

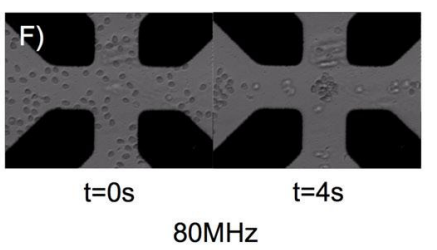

Figure 5.4. 20x Microscopy images of native and modified B- experiencing DEP. The first row shows native erythrocytes at $100 \mathrm{kHz}(\mathrm{A}), 42 \mathrm{MHz}(\mathrm{B})$ and $80 \mathrm{MHz}(\mathrm{C})$, whereas the second row shows $\beta$-galactosidasemodified erythrocytes at the same frequencies (D)-(F). At $100 \mathrm{kHz}$, native (A) and modified (D) both show nDEP. At $42 \mathrm{MHz}$, native erythrocytes (B) experience $\mathrm{nDEP}$ and pDEP (out of focus) while the modified erythrocytes (E) experience very weak nDEP and pDEP as would be expected near the COF. $80 \mathrm{MHz}$ images show nDEP for both (C) and (F). The white arrows point to erythrocytes located in the center or away from the electrodes for $\mathrm{nDEP}(\mathrm{B})$ and near the electrode surface for $\mathrm{pDEP}(\mathrm{E})$.

Experiments were repeated for $\mathrm{B}+, \mathrm{B}-, \mathrm{A}+, \mathrm{A}-, \mathrm{AB}+, \mathrm{O}+$, and $\mathrm{O}-$. Erythrocytes from each blood type transitioned from $\mathrm{nDEP}$ to $\mathrm{pDEP}$ and then back from $\mathrm{pDEP}$ to $\mathrm{nDEP}$ at differing frequencies. $\mathrm{A}+$, for example, experienced both positive and negative dielectrophoresis at $19 \mathrm{MHz}$ (see Figure 5.5A\&B), whereas all of the other native blood types only experienced negative dielectrophoresis at this point. Figure 5.5A shows the ranges over which cells of a given blood type experience nDEP (black bars) and pDEP (gray bars). For the lower cross-over frequency range, this behavior was tabulated for each of the blood types tested in Figure 5.5B. The midpoint of the concurrent $\mathrm{pDEP} / \mathrm{nDEP}$ range was used to estimate cross-over frequency and is shown in Figure 5.6A-D. At 80MHz in Figure 5.5A, it can be seen that all blood types except O- native once again experience negative dielectrophoresis. 
In all blood samples, transitional responses were observed near the cross-over frequencies reflected the distribution of cell differences typical in a real cell population. Red blood cells remain in circulation for around 120 days ${ }^{26}$ and their ribosomal RNA actively synthesize proteins, leading to redistributions in cell density ${ }^{237}$. It is then inferred that polysaccharide distributions in the membrane could also change over the erythrocytes' lifetime. Research has shown that a membrane protein (band 3) plays a role in accelerating erythrocyte aging and also affects anion and glucose transport in the cell. Anion transport across the erythrocyte membrane can be increased as much as three times the normal value ${ }^{177,238}$ and would affect the cytosolic properties such as the conductivity. Real cell populations would have a subset of older erythrocytes with active band 3 and thus altered dielectric properties. Further, oxidative lesions can adhere to older erythrocyte membranes ${ }^{239}$, potentially affecting the membrane's dielectric properties and therefore the dielectrophoretic response. Such properties of a real cell population would alter individual cell's dielectrophoretic response and likely account for the overlapping ranges of $\mathrm{pDEP}$ and $\mathrm{nDEP}$.

To ascertain $\mathrm{ABO}-\mathrm{Rh}$ antigen contributions to the cross-over frequency, four comparisons were made between the data: 1) comparisons between erythrocytes of the same ABO expression, but differing Rh expressions, 2) comparison between the enzyme modified erythrocytes and the $\mathrm{O}$ type with the same Rh expression, 3) comparisons between two donors of the same blood type and 4) comparison between the native (unmodified) erythrocytes and between the enzyme modified erythrocytes.

A comparison between the higher $\mathrm{COF}$ for $\mathrm{B}+$ and $\mathrm{B}-$ (both native) showed that the addition of the Rhesus factor in $\mathrm{B}+$ caused a $7 \mathrm{MHz}$ increase in COF. A similar trend 
can be seen wherein $\mathrm{A}+$ native blood experienced a cross-over frequency at $77 \mathrm{MHz}$ and A- experienced its cross-over frequency at $64.5 \mathrm{MHz}$. Table 5.3 provides the average higher COF for the Rh positive erythrocytes which is $71.5 \mathrm{MHz}$ (native) and $71.1 \mathrm{MHz}$ (modified) while the $\mathrm{Rh}$ negative erythrocytes are $62.3 \mathrm{MHz}$ (native) and $66.0 \mathrm{MHz}$ (modified). The higher COF for O- exceeds $80 \mathrm{MHz}$ and was not included in either average. Assuming $(\mathrm{O}+)_{\mathrm{d}}$ had a $\mathrm{COF}$ of $80 \mathrm{MHz}$ alters the modified $\mathrm{Rh}$ positive average to $72.9 \mathrm{MHz}$ which is still fairly consistent with the native average. The presence of the transmembrane Rhesus factor increased the higher COF and enzyme modification altered the higher COF very little. Given that the Rhesus transmembrane protein structures suggest ammonium transport functionality, cytoplasmic conductivity would be impacted and the insulative characteristics of the membrane would be reduced. Higher frequency DEP responses are typically attributed to cytosol characteristics, which is consistent with these results ${ }^{118}$. 

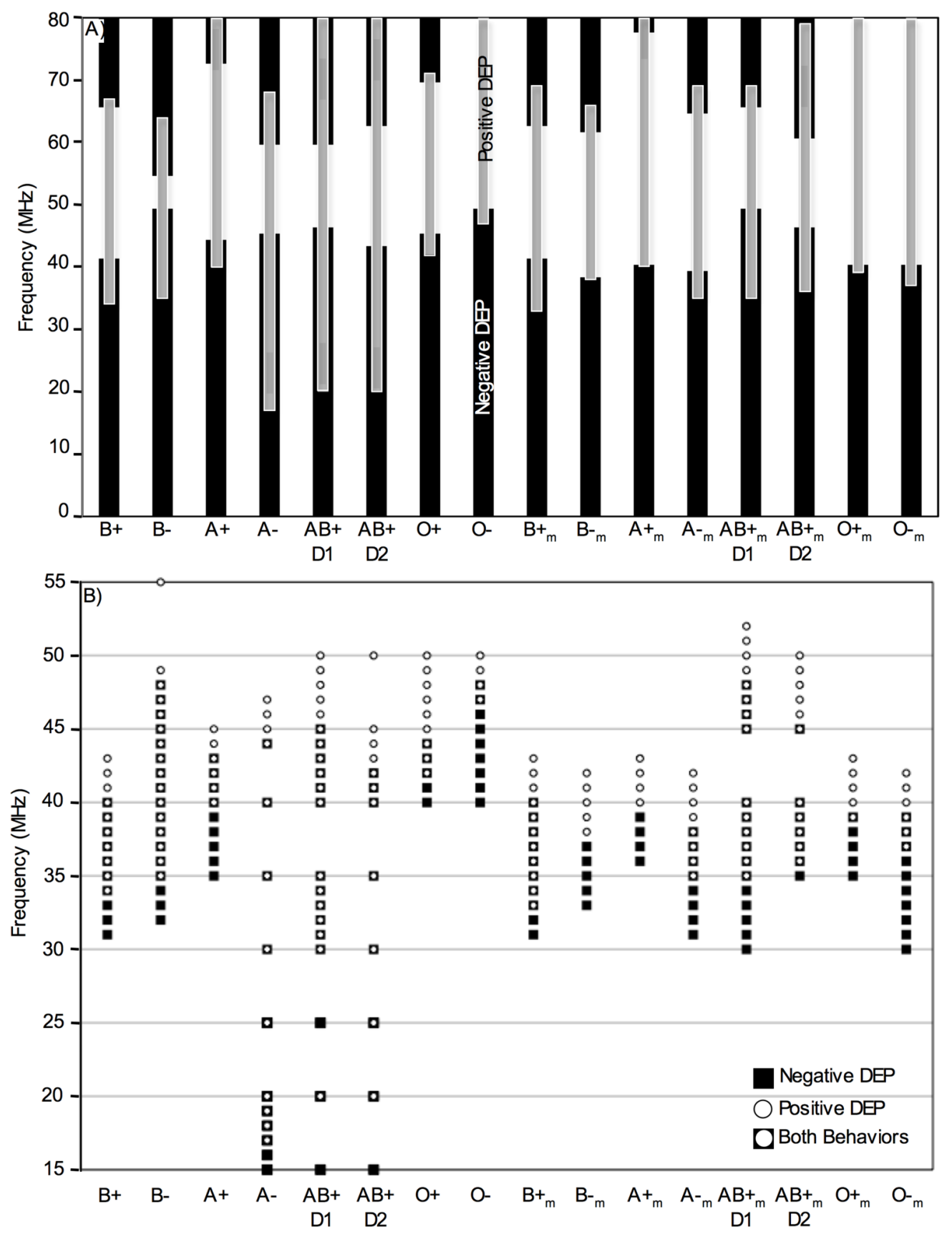

Figure 5.5. (A) Negative (black bars) and positive (gray bars) dielectrophoresis ranges for native (left side) and modified (right side) erythrocytes. COF was calculated as the midpoint of the concurrent $\mathrm{nDEP} / \mathrm{pDEP}$ range. (B) Lower COF range (30-55 MHz) showing nDEP (匹), pDEP ( $\circ$ ) or both $(\square+\circ)$ behaviors. Differences are discernable with $\mathrm{ABO}$ antigen expression.

The lower frequency region is shown in Figure 5.5B. Overlaps in nDEP/pDEP

ranges were observed, but it may be possible to differentiate between $\mathrm{ABO}$ blood types. 
For example, in Figure 5.5B, B+'s combined nDEP/pDEP range was 34 to $40 \mathrm{MHz}$ whereas $\mathrm{O}+$ 's combined range was 42 to $44 \mathrm{MHz}$. This means $\mathrm{B}+$ could be distinguished from $\mathrm{O}+$ with either pure $\mathrm{nDEP}(\mathrm{O}+)$ or pure $\mathrm{pDEP}(\mathrm{B}+)$ at all frequencies in the range. Similarly, A+ could be distinguished from B+ from 34 to $39 \mathrm{MHz}$ and 41 to $44 \mathrm{MHz}$. A+ and $\mathrm{O}+$ would be harder to distinguish however. Further, reproducibility of donors would encroach upon these ranges as demonstrated by $\mathrm{AB}+$ donor 1 and $\mathrm{AB}+$ donor 2 who display a similar broad range of simultaneous pDEP/nDEP (20 to $42 \mathrm{MHz})$ and variation in the onset of pure pDEP behavior ( 42 to $45 \mathrm{MHz}$ ). These separation ranges are greater than any published COF experimental error either directly reported or calculated from graphs: $0.0625 \mathrm{MHz}^{4}, 0.1 \mathrm{MHz}^{240}, 0.475 \mathrm{MHz}^{167}, 1 \mathrm{MHz}^{241}$. In addition, many papers do not report error ${ }^{12,123,124,158,161,162,170}$. A paper with bovine erythrocytes determined a COF shift of $1.5 \mathrm{MHz}$ (no error reported) between cells that were 4 weeks old and 1 week old ${ }^{123}$. Therefore, the frequency shifts observed between blood types are discernable from typical experimental error and consistent magnitude with other DEP shifts.

The cross-over frequency was computed as the midpoint of the simultaneous $\mathrm{nDEP} / \mathrm{pDEP}$ range and is tabulated in Table 5.3. The lower COF range for all ABO-Rh erythrocytes was initially the same as that for the higher $\mathrm{COF}(30.5 \mathrm{MHz}$ to $47.5 \mathrm{MHz}$ vs. 60 to $77 \mathrm{MHz}$ ). However, after modification, the range of the higher COF stayed high at $15 \mathrm{MHz}$ but the lower COF decreased greatly to a range of $5 \mathrm{MHz}$. Figure $5.6 \mathrm{~A} \& \mathrm{~B}$ compare the native and modified samples for $\mathrm{AB}+$ donor $1, \mathrm{O}+$ and $\mathrm{O}-$. Native $\mathrm{AB}+$ experienced a $10.5 \mathrm{MHz}$ lower COF than O+ and a $15 \mathrm{MHz}$ lower COF than O-. However, post-modification traces seen in Figure 5.6B shown a gap of 3.5MHz between 
$\mathrm{O}-$ and $\mathrm{AB}+$ primarily due to an increase in modified $\mathrm{AB}+\mathrm{COF}$ of $9 \mathrm{MHz}$. This indicated that the presence of $\mathrm{A}$ and $\mathrm{B}$ antigens influenced $f_{C M}$ and thus the dielectrophoretic behavior. Figure $6 \mathrm{C}$ demonstrates donor reproducibility regarding this trend between the native and modified erythrocytes. Native $A B+s$ lower COFs differ by $1.5 \mathrm{MHz}$ while the modified $\mathrm{AB}+\mathrm{s}$ differ by $1 \mathrm{MHz}$. Both donors display similar nDEP to pDEP transitions indicating good donor reproducibility. However, more experiments with a panel of donors will need to be conducted to draw conclusions about donor variability.

Figure 5.6D presents the lower cross-over frequency for all native and modified erythrocytes. The lower COF for native erythrocytes (closed symbols) was observed over a $17 \mathrm{MHz}$ range whereas post-modification (open symbols), this range narrowed to $5 \mathrm{MHz}$, seen in Figure 5.6D. Type $\mathrm{O}$ blood antigens have the same polysaccharide backbone as $\mathrm{A}$ and $\mathrm{B}$ so $\mathrm{O}$ antigens were also cleaved at the galactosidase bonds and displayed shifts in their lower COF suggesting that each sugar in the antigen chain contributes to the erythrocyte membrane polarizability and therefore to the cross-over frequency. 

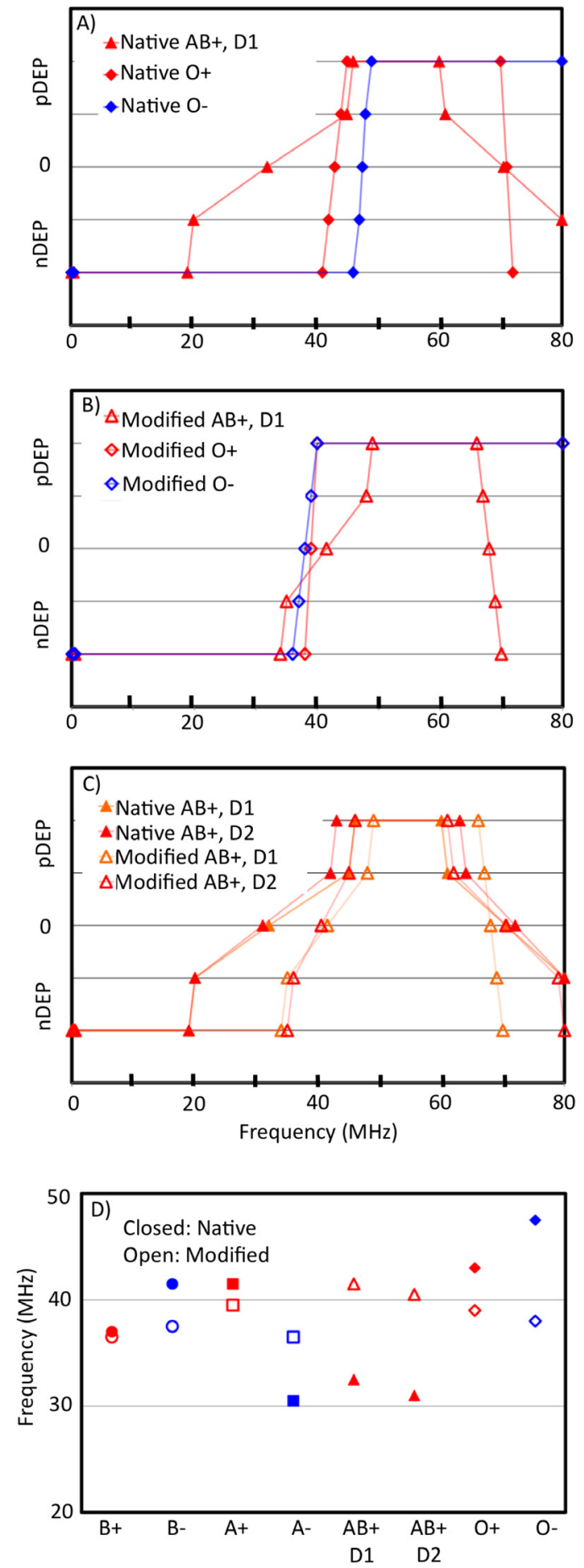

Figure 5.6. A more traditional plot of nDEP, COF and pDEP behaviors as a function of frequency. Plot (A) demonstrates a $\mathrm{COF}$ difference between native $\mathrm{AB}+, \mathrm{O}+$ and $\mathrm{O}$ - that is negated after modification (B). Graph (C) illustrates donor reproducibility between native and modified $\mathrm{AB}+$. (D) The narrowing of the lower COF range from the native (closed symbols) to the modified (open symbols) erythrocytes. 
Table 5.3.

Low and high COF for the native and modified blood types tested. Average, standard deviation and range are computed. Average low and high COF are comparable between native and modified erythrocytes. However, the low COF range for the native erythrocytes is $17 \mathrm{MHz}$, whereas after modification this range drops to $5 \mathrm{MHz}$. The range for the high COF only drops by $2 \mathrm{MHz}$ indicating that this value is not controlled by the expression of

\begin{tabular}{|c|c|c|c|}
\hline \multicolumn{4}{|c|}{ ABO antigens. } \\
\hline & Blood Type & Low COF (MHz) & $\begin{array}{l}\text { High COF } \\
(\mathrm{MHz})\end{array}$ \\
\hline \multirow{13}{*}{ 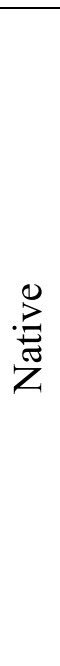 } & $\mathrm{B}+$ & 37 & 67 \\
\hline & B- & 41.5 & 60 \\
\hline & $\mathrm{A}+$ & 41.5 & 77 \\
\hline & A- & 30.5 & 64.5 \\
\hline & $\mathrm{AB}+\mathrm{D} 1$ & 32.5 & 70.5 \\
\hline & $\mathrm{AB}+\mathrm{D} 2$ & 31 & 72 \\
\hline & $\mathrm{O}+$ & 43 & 71 \\
\hline & O- & 47.5 & $>80$ \\
\hline & Average & 38.1 & 68.9 \\
\hline & Standard Dev & 6.28 & 5.54 \\
\hline & Range & 17 & 17 \\
\hline & Rh Positive Average & 37.0 & 71.5 \\
\hline & Rh Negative Average & 39.8 & 62.3 \\
\hline \multirow{13}{*}{ 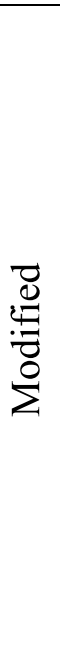 } & $\mathrm{B}+\mathrm{d}$ & 36.5 & 66.5 \\
\hline & B-d & 37.5 & 64.5 \\
\hline & $\mathrm{A}+\mathrm{d}$ & 39.5 & 79.5 \\
\hline & A-d & 36.5 & 67.5 \\
\hline & $\mathrm{AB}+\mathrm{d} \mathrm{D} 1$ & 41.5 & 68 \\
\hline & $\mathrm{AB}+\mathrm{d} \mathrm{D} 2$ & 40.5 & 70.5 \\
\hline & $\mathrm{O}+\mathrm{d}$ & 39 & $>80$ \\
\hline & $\mathrm{O}-\mathrm{d}$ & 38 & $>80$ \\
\hline & Average & 38.6 & 69.4 \\
\hline & Standard Dev & 1.83 & 5.31 \\
\hline & Range & 5 & 15 \\
\hline & Rh Positive Average & 39.4 & 71.1 \\
\hline & Rh Negative Average & 37.3 & 66.0 \\
\hline
\end{tabular}

\subsection{Conclusions}

Portable medical microdevices have made headway into mainstream medicine

with widely recognized potential for ever more versatile, yet precise and accurate biofluid diagnostic devices. The ability to reliably determine ABO-Rh blood type from a drop of human blood sample is an essential, yet fundamental medical diagnosis step. This paper 
explored dielectrophoretic cross-over frequencies of erythrocytes with known $\mathrm{ABO}-\mathrm{Rh}$ antigen expression and compared to the same erythrocytes modified to remove sugar units from the $\mathrm{A}, \mathrm{B}$, and $\mathrm{O}$ antigens. The first part of this study systematically optimized and verified enzyme modification of the human erythrocytes to cleave ABO polysaccharides at the $\beta$ (1-3) galactose bond. We achieved a) acceptable levels of erythrocyte lysis, b) positive identification of the saccharides in the supernatant, and c) no agglutination against the blood type's antibody. It should be noted that this technique is a means to alter non-compatible blood for transfusions into universally donate-able blood. To the author's knowledge, this technique has not been reported in the literature.

The second half of this study utilized a quadrupole dielectrophoretic microdevice to map out regions and strength of negative dielectrophoresis and positive dielectrophoresis for seven of the eight blood types and compared to the same erythrocyte samples after antigen modification. Results demonstrated that erythrocytes a) experienced differing dielectrophoretic forces at a given frequency based on $\mathrm{ABO}-\mathrm{Rh}$ expression that manifested as altered nDEP and pDEP ranges, and $b$ ) the enzyme modified cells exhibited different cross-over frequencies from their corresponding native cells.

The presence of the transmembrane Rhesus factor, (i.e. positive blood types) increased the higher $\mathrm{COF}$ in the 70 to $80 \mathrm{MHz}$ range. This difference was $\sim 9 \mathrm{MHz}$ and greater than any experimental error $(0.0625$ to $1 \mathrm{MHz})$ reported in the literature. This suggests that the DEP shifts could be exploited to trap Rh positive erythrocytes from $\mathrm{Rh}$ negative erythrocytes of the same $\mathrm{ABO}$ type. The Rhesus factor is a transmembrane protein that likely functions as an ion channel thus affecting the membrane permeability 
and supporting prior conclusions that the higher COF is associated with cytosolic properties.

The lower COF signatures in the 40 to $45 \mathrm{MHz}$ range could be exploited to distinguish $\mathrm{ABO}$ blood types. Results were compiled to show ranges over which native A erythrocytes experienced opposite dielectrophoretic behavior from native $\mathrm{O}$ erythrocytes or similarly for A to B and B to O (all native). Erythrocytes with both A and $\mathrm{B}$ antigens displayed unique concurrent $\mathrm{nDEP}$ and $\mathrm{pDEP}$ signatures over a wide frequency range and reproducibility between donors was good. Current results suggest the potential to determine the ABO-Rh blood type of an unknown sample using multistep comparisons.

This work also demonstrated that modification of human erythrocytes with $\beta(1-3)$ galactosidase caused an appreciable change in the lower cross-over frequency. Since the $\beta(1-3)$ galactosidase only reacted with $\mathrm{ABO}$ antigens and would not have altered the $\mathrm{Rh}$ antigens, this result indicated that the lower COF is dependent on membrane surface expression. While average low and high COF are comparable between native and modified erythrocytes, the low $\mathrm{COF}$ range for the native erythrocytes is $17 \mathrm{MHz}$, whereas after modification this range drops to $5 \mathrm{MHz}$. This directly points to $\mathrm{ABO}$ antigen expression strongly influencing dielectrophoretic behaviors. The range for the high COF only drops by $2 \mathrm{MHz}$ indicating that this value is not controlled by the expression of $\mathrm{ABO}$ antigens.

Cross-over frequencies observed in these experiments differ from literature values ${ }^{4,123}$ most likely because our medium conductivities were an order of magnitude larger; theory and experimental results support that cross-over frequency is a strong function of 
medium conductivity ${ }^{8,12,242}$. However, it is probably that lower medium conductivities would completely disguise the polarization effects of the $\mathrm{ABO}-\mathrm{Rh}$ antigens because at lower medium conductivities, the lipid bilayer for a cell represents a molecular-sized capacitor and since this dimension is smaller than the double layer, all double layer effects become unimportant. While this yields the strongest DEP forces, the response is dominated by the membrane's phospholipids and would shield membrane protein affects on the double layer conductivity and permeability. Increasing the external medium conductivity facilitates polarization of the membrane exterior thus screening penetration into the cell [17]. Lastly, advantages exist with performing DEP at physiological conditions close to pure blood plasma $(\sim 1.1 \mathrm{~S} / \mathrm{m})$ because it might be possible to perform DEP diagnostics with minimal dilutions or in vivo. This work also suggests that bovine erythrocyte results may not be as comparable to human erythrocyte responses as previously assumed because bovine blood typing systems differ from human blood types 243 and the erythrocytes likely have different dielectric properties ${ }^{123}$.

In summary, the $\mathrm{ABO}$ antigen structures and chemical compositions play a key role in an erythrocyte's effective polarizability in dielectrophoretic fields. Since ABO antigens are expressed on the membrane surface, they likely impact surface conductivity; whereas the transmembrane Rh-D antigen likely influences the permittivity of the membrane. Thus, A, B or $\mathrm{O}$ antigens affects the dielectric properties of the cell membrane and therefore the dielectrophoretic force on the entire cell at a specified frequency. 


\subsection{Acknowledgements}

All work was performed in an Institutional Biosafety Committee (IBC) certified

Biosafety Level 2 laboratory and received approval from the Internal Review Board (IRB) for the protection of human subjects (Approval \#M0540). Work was supported by the NSF CBET CAREER (0633538/ $\underline{1041338)}$ ) and NSF GRS (0737864, 0841403, 0942772). Thanks to Michael Brodeur-Campbell and Dr. David Shonnard (Chemical Engineering, Michigan Technological University) for their assistance with the HPLC analysis and Sushil Pachpinde and Dr. Ching-An Peng (Chemical Engineering, Michigan Technological University) for their assistance with the Zeta Potential measurements. The authors have declared no conflict of interest. 


\section{Dielectrophoretic Response of Human Erythrocytes Using AC Sweeps: ABO-Rh Antigen \& Conductivity Dependencies}

\subsection{Abstract}

A quadrupole dielectrophoretic microdevice was used to determine $\mathrm{ABO}-\mathrm{Rh}$ dependency on dielectrophoretic (DEP) behavior of human erythrocytes. Previous work examined ABO-Rh impact on dielectrophoretic responses in the $\mathrm{MHz}$ region under $0.9 \mathrm{~S} / \mathrm{m}$ physiological medium conductivity conditions. In contrast, this work utilizes sweeping frequency instrumentation to explore the dielectrophoretic behavior of native and $\beta(1-3)$ galactosidase modified human erythrocytes at conductivities of $0.01 \mathrm{~S} / \mathrm{m}, 0.04 \mathrm{~S} / \mathrm{m}$ and $0.1 \mathrm{~S} / \mathrm{m}$. Erythrocyte dielectrophoretic behaviors were observed via video microscopy and quantified via intensity analysis over $10 \mathrm{kHz}$ to $80 \mathrm{MHz}$ with a focus on the frequency range from $10 \mathrm{kHz}$ to $1.9 \mathrm{MHz}$. Four intensity profiles in the symmetric quadrupole device were used to approximate magnitudes of negative dielectrophoresis (nDEP) and positive dielectrophoresis (pDEP) behaviors as a function of frequency. The frequency sweep and automated intensity analysis to track cell motion allowed for DEP spectra to be obtained for each $\mathrm{ABO}-\mathrm{Rh}$ blood type alongside the $\mathrm{ABO}$ modified erythrocyte. Crossover frequencies were also extracted from the DEP spectra. ABO-Rh antigen expression and modification are shown to influenced cell polarization and therefore dielectrophoretic behavior. Further, the resolution between DEP blood type responses is exacerbated at conductivities below the $0.9 \mathrm{~S} / \mathrm{m}$ conductivities previously published. 


\subsection{Introduction}

The notion of dielectrophoresis began with Pohl ${ }^{15}$, but it has been used to determine differences between particles ${ }^{8,17,157,158,160,164,166,167,175,183-186}$, similar cell types $^{1,7-9,11-14}$ and healthy versus infected cells ${ }^{3-6}$. One commonality is that these studies use single frequency trials to determine the dielectrophoretic and electrorotation behavior of particles and cells $\mathrm{s}^{1,3-9,11-14,17,118,123,124,157-162,164,166,167,175,183-186,188-192}$. This work builds upon a novel, but limitedly explored, method to explore frequency-dependent dielectrophoretic responses by applying an alternating current frequency sweep to a cell sample. Further, a novel automated intensity profile analysis was developed and coupled with the frequency sweeps to enable the quantification of full DEP spectral behaviors, which can then be fit to the theoretical equations.

Alternating current dielectrophoresis (AC DEP) is the movement of particles in an spatially inhomogeneous electric field. A particle will experience either positive dielectrophoresis and move towards regions of high field density or negative dielectrophoresis and move towards regions of low field density based on the particle's intrinsic polarizability compared to the suspending medium ${ }^{15}$. Full explanation of the theory behind AC DEP can be found in Chapter 3. The DEP force equation for an ellipsoidal particle with a shell, which applies to a human erythrocyte and its membrane is:

$$
\left\langle\vec{F}_{D E P, i}(t)\right\rangle=2 \pi a_{1}^{2} a_{3} \varepsilon_{m} \operatorname{Re}\left[f_{C M, i}(\omega)\right] \nabla E
$$

where the Clausius-Mossotti factor, $f_{C M, I}$ is given by: 


$$
f_{C M, i}^{*}=\frac{\varepsilon_{p}^{*}-\varepsilon_{m}^{*}}{3\left[\varepsilon_{m}^{*}+\left(\varepsilon_{p}^{*}-\varepsilon_{m}^{*}\right) L_{i}\right]}
$$

The depolarization factor, $L_{i}$, accounts for the eccentricity of the cell due to its nonspherical shape is axis dependent as follows.

$$
L_{i}=\frac{a_{1}^{2} a_{3}}{2} \int_{0}^{\infty} \frac{1}{\left(l+a_{i}^{2}\right) \sqrt{\left(l+a_{1}^{2}\right)^{2}\left(l+a_{3}^{2}\right)}} d l
$$

Equations (2) and (3) account for the ellipsoidal geometry of human

erythrocytes $^{4,114,115,123,124,162}$ and Equation (4) accounts for the thin shell approximation of the Core-Shell model applicable to biological cells with thin membranes ${ }^{114,115,117}$.

$$
\varepsilon_{p}^{*}=\left[\frac{\varepsilon_{c}^{*} \varepsilon_{s}^{*}}{\frac{\Delta a}{a_{s}} \varepsilon_{c}^{*}+\varepsilon_{s}^{*}}\right]
$$

Dielectrophoresis is beneficial in microfluidic devices because manipulated particle sizes are on the order $1 \mu \mathrm{m}$ whereby the dielectrophoretic force dominates over the gravitational force and Brownian motion ${ }^{117}$. For human erythrocyte at $\sim 7 \mu \mathrm{m}$ diameter, particle displacement in 1 second is approximately $10 \mathrm{~nm}$ due to the gravitational force and $1 \mu \mathrm{m}$ due to Brownian motion, according to Morgan and Green ${ }^{117}$. The dielectrophoretic force displacement is dependent on characteristic electrode dimensions which govern the electric field gradient; at the $2.5 \mathrm{~V}_{\mathrm{pp}}$ in our devices, and are thus dominant over the other two forces ${ }^{117}$. 
The goal of most dielectrophoretic microdevices is to discern one cell type from another. Researchers have distinguished healthly from Plasmodium falciparum infected human erythrocytes and determined the altered dielectric parameters through a combination of field-flow fractionation DEP and electrorotation ${ }^{4,5}$. DEP has also successfully separated leukemia cells experiencing trapping nDEP from healthy blood cells that experience pressure-drive flow ${ }^{3}$ near zeroDEP at $3 \mathrm{MHz}$. Different subpopulations of leukocytes displayed differing dielectric properties ${ }^{12-14}$. Similar to erythrocytes, these cells express morphological differences, but they are derived from a common progenitor cell and therefore have similar biochemical properties. The commonality between leukocyte subpopulations was discussed in Chapter 2. An impedance-based approach to leukocyte subpopulation cell identification and separation occurred, however these differences were not linked to any biochemical or biophysical cell properties beyond the traditionally reported dielectric properties of membrane permittivity and conductivity ${ }^{12}$.

Prior research has largely overlooked healthy erythrocyte blood type ${ }^{5,23}$. Recent work published by this group revealed differing ABO-Rh human erythrocytes behaviors in $\mathrm{AC} \mathrm{DEP}^{1,11}$ and DC DEP fields ${ }^{10}$. The human erythrocyte system is optimal for exploring the limits of DEP-discernable cell attributes. Common properties of all erythrocytes are cytosolic and membrane composition as discussed in Chapter 2. They differ in phenotype by blood type of which the ABO surface antigens and $\mathrm{Rh}$ transmembrane antigens are key. Thus, this work is able to explore how changes in expression of saccharide functional groups or removal of the saccharide altogether impact whole cell polarizability in DEP fields. 
This work moves beyond fixed frequency experiments into frequency sweeps to determine the dielectric properties of $\mathrm{ABO}-\mathrm{Rh}$ human erythrocytes as a function of frequency, conductivity, and ABO modification. Cell motions in the DEP field were tracked with intensity profiles and automated analysis to obtain experimentally derived DEP profiles as a function of frequency for direct comparison with theoretical profiles as shown in Chapter 3. This approach facilitates model comparison to experimental DEP curves via a least squares approach yielding more accurate dielectric property predictions than from two COF values. This greater accuracy has allowed this research to distinguish between multiple ABO-Rh cell types, which is an 8 dimensional discernment of cell phenotype, and a large advance above the biomodal distinctions with prior work regarding healthy/unhealthy ${ }^{2-7}$ and similar cell types ${ }^{7-9,12-14}$.

\subsection{Materials and Methods}

\subsubsection{Materials}

Human erythrocytes of type $\mathrm{A}+, \mathrm{A}-, \mathrm{B}+, \mathrm{B}-, \mathrm{AB}+, \mathrm{AB}-, \mathrm{O}+$ and $\mathrm{O}-$ were obtained from human blood samples of voluntary donors with verified blood type via a Portage Health Clinic phlebotomist at Michigan Technological University. Four donors for each blood type were recruited and one donor was asked to repeat the donation greater than 5 days later such that variations between $\mathrm{ABO}-\mathrm{Rh}$ type donors and within same donors could be independently tracked. The blood was stored at $4{ }^{\circ} \mathrm{C}$ and experiments completed within 48 hours of donation to avoid storage-induced changes in the phospholipid composition of the membrane $\mathrm{e}^{231}$ or alterations to $\mathrm{pH}$, hemolysis, $\mathrm{K}+$ concentration and 
erythrocyte morphology ${ }^{232}$. Erythrocyte polarizability has been reduced with storage, further influencing dielectrophoretic behavior ${ }^{23}$. Three suspending mediums were made from four components (Table 6.1) with final conductivities of $0.01 \mathrm{~S} / \mathrm{m}, 0.04 \mathrm{~S} / \mathrm{m}$ and $0.1 \mathrm{~S} / \mathrm{m}$ after $\mathrm{pH}$ adjustment with $1 \mathrm{M} \mathrm{NaOH}$ or $1 \mathrm{M} \mathrm{HCl}$ to $7.00 \pm 0.03$. The $1 \mathrm{M}$ salt stock was comprised of $0.38 \mathrm{~g} \mathrm{NaCl}, 0.64 \mathrm{~g} \mathrm{KH}_{2} \mathrm{PO}_{4}$, and $0.86 \mathrm{~g} \mathrm{~K}_{2} \mathrm{HPO}_{4}$ in $5 \mathrm{~mL}$ E-pure water. Bovine Serum Albumin (BSA) was included to prevent erythrocyte adherence to the bottom glass slide during experiments. Previous experiments presented in Chapter 5 were at $0.9 \mathrm{~S} / \mathrm{m}^{1}$, similar to pure plasma physiological conductivities of $1.57 \mathrm{~S} / \mathrm{m}$ (predictive equation) ${ }^{133}$ and $1.1 \mathrm{~S} / \mathrm{m}^{1}$ (experimentally measured). Traditionally, DEP experiments were performed at conductivities below $0.1 \mathrm{~S} / \mathrm{m}^{3,4,8,140,162,164,167,226,227,233}$, so experiments presented herein were performed at similar conductivities in order to determine where the $\mathrm{ABO}-\mathrm{Rh}$ antigen dependency fits into the current literature on cellular DEP.

Table 6.1.

Table describing the reactants used in creating the four different medium solutions used. Also included is the

\begin{tabular}{|c|c|c|c|c|c|}
\hline $\begin{array}{l}\text { Solution } \\
\text { Name }\end{array}$ & $\begin{array}{l}\text { 1M Salt } \\
\text { Stock }\end{array}$ & Dextrose & $\begin{array}{c}\text { E-pure } \\
\text { Water }\end{array}$ & $B S A$ & $\begin{array}{c}\text { Final } \\
\text { Conductivity }\end{array}$ \\
\hline $\begin{array}{c}0.01 \mathrm{~S} / \mathrm{m} \\
\text { with } 0.75 \% \\
\text { BSA }\end{array}$ & $4.55 \mu \mathrm{L}$ & $0.8 \mathrm{~g}$ & $14.995 \mathrm{~mL}$ & $0.12 \mathrm{~g}$ & $0.02 \mathrm{~S} / \mathrm{m}$ \\
\hline $\begin{array}{c}0.04 \mathrm{~S} / \mathrm{m} \\
\text { with } 1.5 \% \\
\text { BSA }\end{array}$ & $9.48 \mu \mathrm{L}$ & $0.78 \mathrm{~g}$ & $14.991 \mathrm{~mL}$ & $0.24 \mathrm{~g}$ & $0.04 \mathrm{~S} / \mathrm{m}$ \\
\hline $0.1 S / m$ & $38.6 \mu \mathrm{L}$ & $0.75 \mathrm{~g}$ & $14.961 \mathrm{~mL}$ & $0.0 \mathrm{~g}$ & $0.09 \mathrm{~S} / \mathrm{m}$ \\
\hline $\begin{array}{c}0.1 \mathrm{~S} / \mathrm{m} \text { with } \\
1.5 \% \mathrm{BSA}\end{array}$ & $38.6 \mu \mathrm{L}$ & $0.75 \mathrm{~g}$ & $14.961 \mathrm{~mL}$ & $0.24 \mathrm{~g}$ & $0.11 \mathrm{~S} / \mathrm{m}$ \\
\hline
\end{tabular}




\subsubsection{Microdevice Fabrication}

Microdevices were fabricated via previously published procedures ${ }^{1}$ including micropatterned electrodes, SU-8 soft photolithography and PDMS casting as show in Figure 6.1A-F. Positive photoresist (Futurrex PR-1000A, Franklin, NJ) was spun onto glass slides (Laurell Technologies, North Wales, PA) and UV exposed (EVG620, EVG, AZ) via soft photolithography ${ }^{33,223,224}$ for the micropatterned electrode template. Glass slides were sputter coated with a thin adhesive titanium followed by a gold layer to a final depth of 100nm (Perkin-Elmer Randex Sputtering System Model 2400, Perkin-Elmer, USA). The extra photoresist and sputtered metals were removed from the glass slide by acetone (VWR) sonication yielding the electrode pattern in Figure 6.1D.

The PDMS fluidic layer were fabricated from SU-8 2025 (Microchem, MA, USA) spun onto a 4 inch Si wafer (WRS Materials, CA, USA) to approximately $70 \mu \mathrm{m}$ followed by UV exposure at 20W for 10 seconds (Figure 6.1A). Castings were made of the wafer with a 10:1 PDMS elastomer to curing agent ratio (Dow Corning, USA) and set at $60^{\circ} \mathrm{C}$ for 3 hours (Isotemp Model 280A, Fisher Scientific, Pittsburgh, PA). The casting was removed (Figure 6.1E), cut to size and ports drilled for electrode connections and sample inlet/outlet, then the casting and electrode-patterned glass slide were exposed to oxygen plasma (Harrick Plasma PDC-001, Harrick Plasma, Ithaca, NY) for 30 seconds prior to glass slide/casting alignment, sealing, and placement in a $60^{\circ} \mathrm{C}$ oven for 3 hours to ensure PDMS/glass adhesion. Copper wires were silver epoxy-ed to the plated electrodes and hardened at $90^{\circ} \mathrm{C}$. The final device is in Figure 6.1F. 

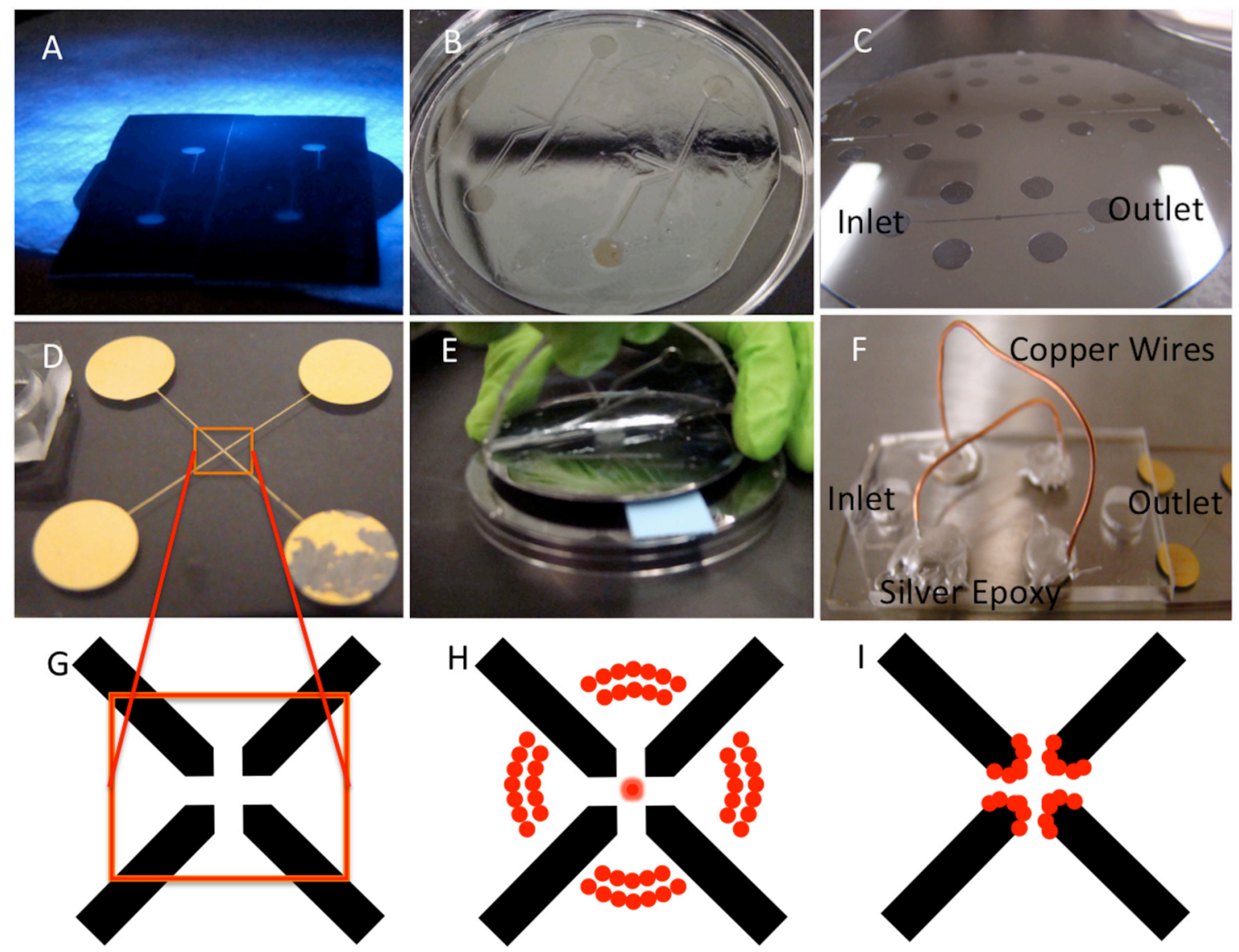

Figure 6.1. Fabrication of microdevices: UV exposure of SU-8 covered silica wafer with UV mask (A), developing of photoresist (B), final wafer showing quadrapole chamber design (C), titanium-gold plated electrodes made via sputter system (D), PDMS casting of chamber (E) and final device showing copper wires used to connect to alligator clips and silver epoxy that connects copper wires to plated electrodes (F). Zeiss view window (for 25X magnification) of electrode area (G). Example of behavior for nDEP (H) and pDEP (I) response.

\subsubsection{Antigen Modification}

In order to compare presence and absence of $\mathrm{ABO}$ antigens on dielectrophoretic behavior, the erythrocytes where enzymatically modified to remove the A, B and O antigens. $\beta(1-3)$ galactosidase (New England Biolabs, Ipswich, MA) breaks the $1 \rightarrow 3$ galactosidase bonds with the following reactants: $66.7 \%$ packed erythrocytes, $0.1 \mathrm{U} / \mu \mathrm{L}$ $\beta(1-3)$ galactosidase, $10 \% \mathrm{G} 2$ reaction buffer, $1 \%$ bovine serum albumin, and $21.3 \%$

$\mathrm{NaCl}$ (VWR) (volume to total volume percentages). For full experimental procedure 
regarding antigen modification see Figure 1 in Leonard et al, $2011^{1}$ which is reprinted in Chapter 5 as Figure 5.1.

\subsubsection{Dielectrophoresis Experiments}

For each blood donation, four erythrocytes dilutions were prepared with the supporting mediums in Table 6.1. Whole blood was centrifuged at 1400rpm for 5 minutes and washed in $0.9 \% \mathrm{NaCl}$ solution to recover packed blood cells for $1: 75 \mathrm{~V}: \mathrm{V}$ resuspension in $0.01 \mathrm{~S} / \mathrm{m}$ with $0.75 \% \mathrm{BSA}$ as well as in $0.1 \mathrm{~S} / \mathrm{m}$ solutions. The enzymatically modified blood cells were suspended in a 1:25 V:V ratio with $0.04 \mathrm{~S} / \mathrm{m}$ with $1.5 \%$ BSA or $0.1 \mathrm{~S} / \mathrm{m}$ with $1.5 \% \mathrm{BSA}$; the differing dilutions was to achieve comparable visual cell densities between the native and modified experiments. BSA was used as a means to prevent the cells from sticking to the glass slide of the microdevice. Samples were individually injected into the microdevice using a modified LabSmith configuration with sample reservoirs and syringes. New samples were injected for each sweep run and washing with E-pure water occurred before switching to a new solution type. 
Table 6.2.

24 sweep (six per solution) that were run for these experiments. Notice that the low conductivity solutions were run with the same six sweeps and the higher conductivity solutions were run with another set of six sweeps. For each solution two of the sweeps were repeated, explaining why two sweeps for each set show up twice.

\begin{tabular}{|c|c|c|c|c|}
\hline Solution & $\begin{array}{c}\text { Start Frequency } \\
(\mathrm{MHz})\end{array}$ & $\begin{array}{l}\text { End Frequency } \\
(\mathrm{MHz})\end{array}$ & Time (sec) & Rate \\
\hline $\begin{array}{l}0.01 \mathrm{~S} / \mathrm{m} \text { with } \\
0.75 \% \mathrm{BSA} \\
\text { and } \\
0.04 \mathrm{~S} / \mathrm{m} \text { with } \\
1.5 \% \mathrm{BSA}\end{array}$ & $\begin{array}{l}0.01 \\
0.01 \\
0.01 \\
0.01 \\
0.01 \\
0.01\end{array}$ & $\begin{array}{l}0.91 \\
1.81 \\
0.235 \\
0.460 \\
1.81 \\
0.91\end{array}$ & $\begin{array}{l}200 \\
400 s \\
100 \\
400 \\
400 \\
200\end{array}$ & $\begin{array}{l}2 \mathrm{x} \\
2 \mathrm{x} \\
1 \mathrm{x} \\
0.5 \mathrm{x} \\
2 \mathrm{x} \\
2 \mathrm{x}\end{array}$ \\
\hline $\begin{array}{l}0.1 \mathrm{~S} / \mathrm{m} \\
\text { and } \\
0.1 \mathrm{~S} / \mathrm{m} \text { with } \\
1.5 \% \mathrm{BSA}\end{array}$ & $\begin{array}{l}0.1 \\
0.1 \\
0.1 \\
0.1 \\
0.1 \\
0.1\end{array}$ & $\begin{array}{l}1 \\
1 \\
1.9 \\
0.55 \\
1 \\
1\end{array}$ & $\begin{array}{l}200 \\
400 \\
400 \\
200 \\
200 \\
400\end{array}$ & $\begin{array}{l}2 \mathrm{x} \\
1 \mathrm{x} \\
2 \mathrm{x} \\
1 \mathrm{x} \\
2 \mathrm{x} \\
1 \mathrm{x}\end{array}$ \\
\hline
\end{tabular}

Dielectrophoretic experiments were performed using an $\mathrm{AC}$ waveform generator (Agilent 33250A, Agilent, Santa Clara, CA) to apply a $2.5 \mathrm{~V}_{\mathrm{pp}}$ sinusoidal electrical signal. The frequency was swept across a range of frequencies at the rates outlined in Table 2. instead of being fixed as is normal in dielectrophoretic experiments. Images were recorded every second with a Zeiss Axiovert 200M inverted light microscope (Zeiss) at 10x magnification with a 2.5 optovar achieving the field of view shown by the red box in Figure 6.1G. Figure 6.1H and Figure 6.1I show cartoons of nDEP and pDEP behavior, respectively. The respective behavior is governed in sign by equation (2) and in magnitude by equation (1). The raw video images for example $\mathrm{A}$ - and $\mathrm{O}$ - donations at $0.1 \mathrm{~S} / \mathrm{m}$ swept from $100 \mathrm{kHz}$ to $1.9 \mathrm{MHz}$ over 400 seconds are shown in Figure 6.2. The transitional region from $\mathrm{nDEP}$ to $\mathrm{pDEP}$ behavior is different for the A- and O- blood 
types shown. Limited experiments were performed from $1.9 \mathrm{MHz}$ to $80 \mathrm{MHz}$ and only nDEP was observed with all blood types for both 0.1 and $0.01 \mathrm{~S} / \mathrm{m}$. Data is not shown.

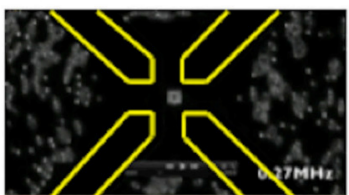

nDEP

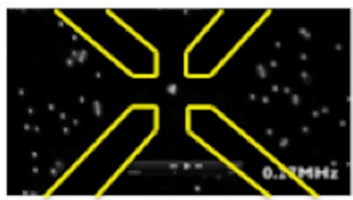

nDEP

A. Native A-suspended in $0.15 / \mathrm{m}$ dextrose buffer

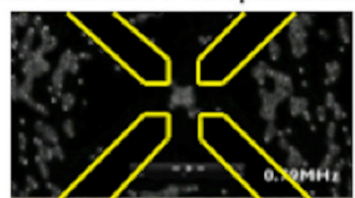

Transition

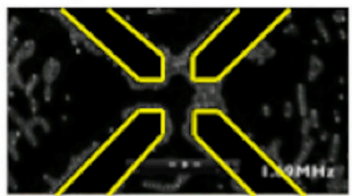

pDEP

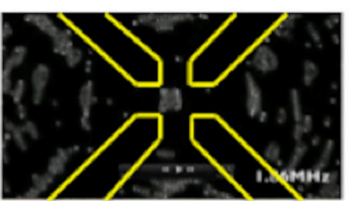

$\mathrm{nDEP}$

B. Native $\mathrm{O}$ - suspended in $0.1 \mathrm{~S} / \mathrm{m}$ dextrose buffer

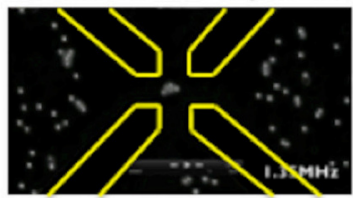

Transition

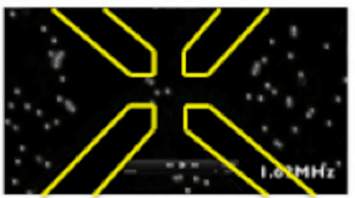

pDEP

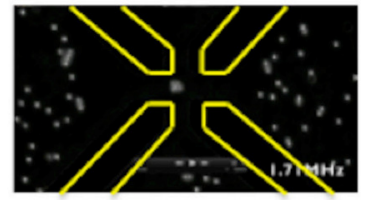

nDEP

Figure 6.2. Native (A) and $O$ - (B) erythrocytes suspended in $0.1 \mathrm{~S} / \mathrm{m}$ dextrose buffer and subjected to a $2.5 \mathrm{~V}_{\mathrm{pp}}$ signal swept from $100 \mathrm{kHz}$ to $1.9 \mathrm{MHz}$ over 400 seconds. At $0.27 \mathrm{MHz}$ both samples are experiencing $\mathrm{nDEP}$. The transition to pDEP occurs at $0.79 \mathrm{MHz}$ for $\mathrm{A}$ - and $1.35 \mathrm{MHz}$ for $\mathrm{O}$-. A period of strong pDEP occurs at $1.69 \mathrm{MHz}$ for $A$ - and $1.62 \mathrm{MHz}$ for $O$-. Transition back to $\mathrm{nDEP}$ occurs at $1.86 \mathrm{MHz}$ for $\mathrm{A}$ - and $1.71 \mathrm{MHz}$ for $\mathrm{O}$-. This demonstrates visually observable differences in blood cell motion by ABO type.

\subsubsection{Intensity Profile Analysis}

In order to independently quantify both the sign (Equation 2) and the magnitude (equation 1) of the dielectrophoretic behavior, image intensity was used to discern cell location and thus motion with time. The frequency sweep and automated intensity analysis enabled DEP spectra to be obtained for each native $\mathrm{ABO}-\mathrm{Rh}$ and corresponding ABO-modified erythrocyte sample. Five intensity profiles were used to accurately track cell motion in the electric field gradient and thus obtain dielectrophoretic direction and extent of cell population response as a function of frequency. The placement of four lines is shown in Figure 6.3: upward, downward, horizontal and vertical. The fifth line captured image background to then calculate intensity deviations. A matrix was constructed from these each intensity profile; the matrix components were $\mathrm{x}$ and $\mathrm{y}$ 
position, corresponding intensity value for each position and time (corresponds to frequency).
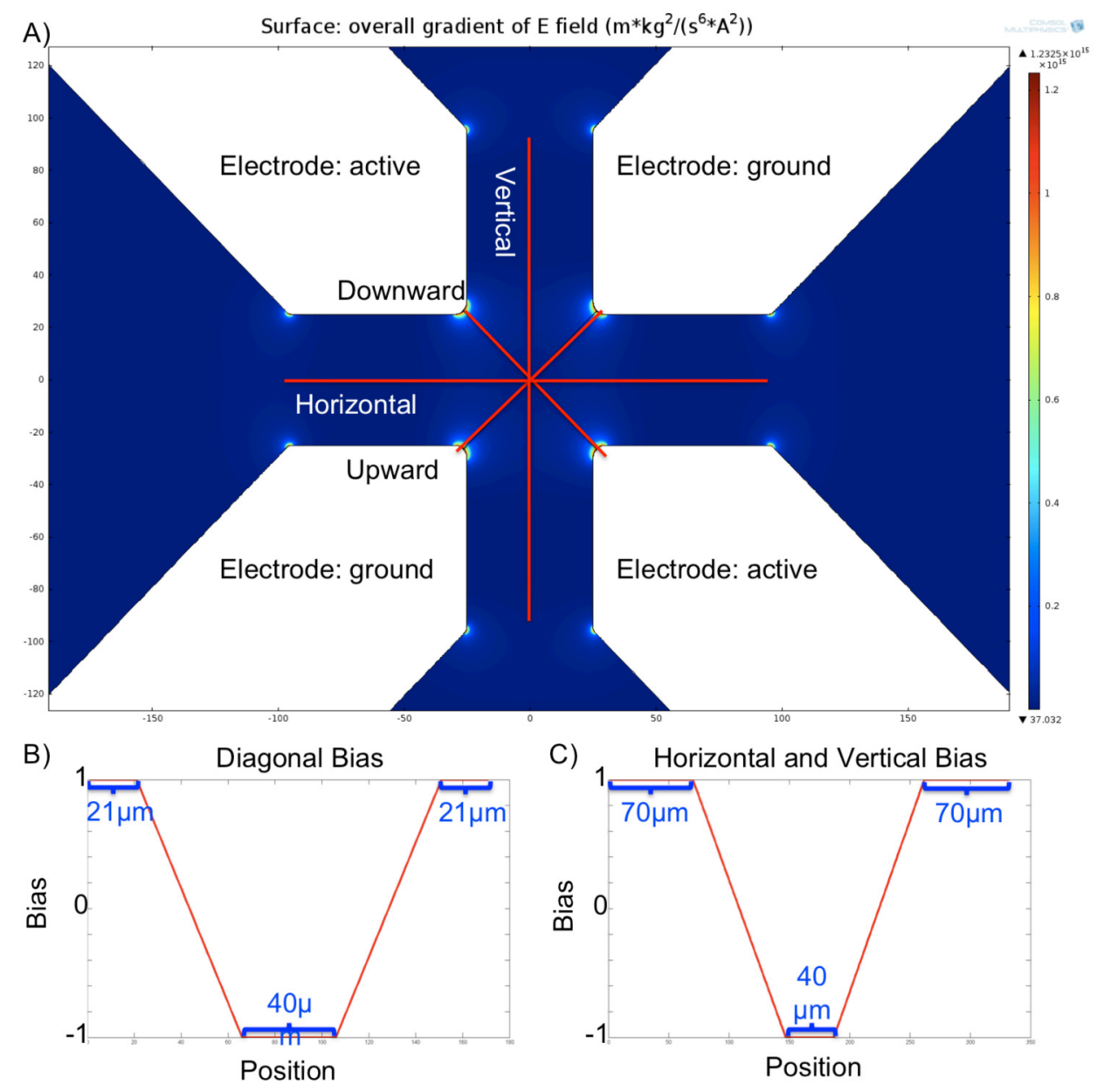

Figure 6.3. (A) COMSOL simulation of of gradient of Electric field squared for quadrapole device showing Downward, Upward, Horizontal and Vertical profile line positions. Diagonal (B) and Horizontal/Vertical Bias (C) used in the calculation of the biased intensity from the raw intensity values.

MATLAB code (included as Appendix 9.B) was custom written to combine these four matrices for a single experiment into a single DEP spectral curve as a function of frequency. The following describes the mathematical manipulations written into the code.

Each profile line, $\mathrm{p}$, for a sweep, $\mathrm{s}$, is a series of intensity values, $I_{s, p, j, k}$, that are a function of position, $\mathrm{j}$, and frequency tracked via timepoint, $\mathrm{k}$. Sometimes, a cell would 
adhere at a given position on the glass slide and not move in the DEP field the entire experiment. These cells would inaccurately bias the intensity profile. These points were excluded from the profile line by obtaining the standard error for each position across all time points:

$$
\text { StError }_{s, p, j}=\frac{\operatorname{StDev}\left(I_{s, p, j, k}\right)}{k_{\max }}
$$

where $k_{\max }$ was the maximum timepoint. Three standard error thresholds were tested: 0.5 , 0.25 , and 0.1 . If the standard error of a given position was found to be less than the threshold, this was considered indicative of stationary cells and then the intensity values for that position over all timepoints were set equal to the average background intensity for that sweep, Int $t_{s, b a c k g r o u n d}$. Positions with StError $_{\mathrm{s}, \mathrm{p}, \mathrm{j}}$ greater than the threshold retained their original values. An initial standard error threshold of 0.5 was ascertained from experiments without BSA wherein cells stuck to the bottom glass of the microdevice yielding no cell movement and experiments with continuous cell movement. Scrutiny of the code with recent experiments revealed that this standard error threshold was too stringent and thus neglecting key cell (intensity) events. Thus, the additional 0.25 and 0.1 standard error thresholds were examined as shown in Figure 6.5. An optimal threshold lies between 0.25 and 0.1 , but this needs to be itteratively verified with a sample intensity profile from each blood type to ensure that non-moving cells are neglected, but slow moving cells are included.

Once stationary cells are neglected, the deviation from the background intensity (fifth profile line) at each position was calculated and is shown in Figure 6.5: 


$$
\operatorname{Int}_{s, p, j, k}=\left|I_{s, p, j, k}-\operatorname{Int} t_{s, \text { background }}\right|
$$

Next, the applicable diagonal or horizontal/vertical bias line was utilized to transform the position dependent intensity into the dielectrophoretic behavior scale. This bias line was used to designate intensity increases towards either pDEP given a value of +1 , or nDEP given a value of -1 based on location. These lines were constructed assuming a $40 \mu \mathrm{m}$ circle at the quadrapole electrode center was -1 for the strongest nDEP behavior. Similarly, the two diagonal bias had a $21 \mu \mathrm{m}$ region at the tip of each electrode designated +1 because this is where the strongest pDEP behavior occurs. For the vertical and horizontal profiles, the space in between the two electrodes, $70 \mu \mathrm{m}$ on each side, is also each to +1 for strong pDEP. The bias line also includes linear transitions from +1 to -1 along the intensity lines $B_{p, j}$ as depicted in Figure $6.3 \mathrm{~B} \& \mathrm{C}$. Thus, the intensity at each position was multiplied by its corresponding bias value and normalize by the average intensity of the entire time and position profile matrix:

$$
B \operatorname{Int}_{s, p, k}=\frac{\sum_{j} B_{p, j} * \operatorname{Int}_{s, p, j, k}}{\frac{\sum_{j} \sum_{k} \operatorname{Int} t_{s, p, j, k}}{j_{\max } * k_{\max }}}
$$

where $j_{\max }$ is the total number of position points and $\mathrm{k}_{\max }$ is the total number of time points. This provided a biased intensity value for each timepoint along each of the four profiles such that each position had its own DEP estimate versus time curve. This curve was smoothed to remove noise using a 5 point running average over time. To translate 
this curve into DEP estimate versus frequency, the frequency was calculated from the time stamp according to:

$$
\text { freq }_{s, k}=\frac{E F-S F}{T-1} k+S F
$$

where $\mathrm{EF}$ is the end frequency in $\mathrm{MHz}, \mathrm{SF}$ is the starting frequency in $\mathrm{MHz}$ and $\mathrm{T}$ is the time duration of the sweep (see Table 6.2). The first timepoint was always set equal to a frequency of $0 \mathrm{MHz}$ because the sweep was started $\sim 2$ seconds after the video microscope acquired the first image. Thus the first point was neglected and this equation is valid for subsequent timepoints.

In order to get one combined DEP estimate versus frequency profile for a single experiment the four profiles are averaged:

$$
C I n t_{s, k}=\frac{\sum_{p=1}^{4} B \operatorname{Int} t_{s, p, k}}{4}
$$

The maximum and minimum values of the combined intensity were also tabulated by the Matlab program under the nomenclature $C I n t_{\mathrm{s}, \max }$ and $C I n t_{\mathrm{s}, \mathrm{min}}$. The first five timepoints were excluded from the maximum and minimum calculations because the cells were settling into their behavior so the intensity values did not provide a true representation of the behavior occurring at those frequencies.

For each solution type, as given in Table 6.1, there were six sweeps run, as shown in Table 6.2 yielding six combined intensity profiles. The previous work described through Equation (5) was written in Matlab as an executable function. The following work was written in the main code and allowed for the user to input information for all 
six sweeps for a given solution type within a blood type. The overall maximum and minimum intensity values when all six sweeps were considered simultaneously was determined by combining individual $C$ Int $t_{\mathrm{s}, \max }$ and $C$ Int $t_{\mathrm{s}, \min }$ into separate maximum and minimum matrices. The global maximum, GlobalInt ${ }_{\max }$, and minimum, Globalint ${ }_{\min }$, were found by then taking the maximum and minimum of those matrices, respectively. This was done to consistently scale the combined intensity profiles for each sweep so that the maximum intensity value was 100 and the minimum intensity value was -100 . In order to preserve the cross over frequency, positive intensity values were scaled separately from negative intensity values. The scaling values for the positive and negative intensity values for each sweep were given by Equation (6) and (7), respectively:

$$
\begin{aligned}
& \text { pscale }_{s}=\frac{100}{\text { GlobalInt }_{\text {max }}} \text { CInt }_{s, \text { max }} \\
& \text { nscale }_{s}=\frac{-100}{\text { GlobalInt }_{\text {min }}} \text { CInt }_{s, \text { min }}
\end{aligned}
$$

In order to scale each sweep to the same maximum and minimum intensity and preserve the differences between the strength of $\mathrm{pDEP}$ and $\mathrm{nDEP}$ behavior between the sweeps, Equations (8) was used for the positive and negative intensity values, respectively:

$$
\operatorname{SInt}_{s, k}=\left\{\begin{array}{l}
\text { Int }_{s, k} * \frac{\text { pscale }_{s}}{\text { CInt }_{s, \max }}, \text { CInt }_{s, k} \geq 0 \\
\operatorname{CInt}_{s, k} * \frac{n \text { scale }_{s}}{\text { Int }_{s, \text { min }}}, \text { CInt }_{s, k} \leq 0
\end{array}\right.
$$


This results in a scaled DEP response versus frequency curve for each sweep in a given solution type. The sweeps were run at different rates as shown in Table 6.3. The sampling rate, or the number of images per $\mathrm{MHz}$, was used to match the faster sampling rates via 2 or 4 point averaging to the slowest sampling rate at 223 images per $\mathrm{MHz}$, thus combining six sweeps into one set of frequency-DEP response data for a given solution. These sweeps were not combined in a straight average, but rather more weight was given to the sweeps that sample at a higher rate over the lower frequency range because these sweeps are more indicative of behavior in that region. This yielded a single DEP response curve derived from four intensity profile lines in six frequency sweeps. Comparisons were made between blood types, donors within the blood types, repeat donor samples, ABO modification, and medium conductivity.

\subsection{Results}

Four different intensity profiles were found for every timepoint in the frequency sweep. An example of the top left to bottom right profile for an $\mathrm{A}+$ donor at $0.1 \mathrm{~S} / \mathrm{m}$ is shown in Figure 6.4 at four timepoints and thus frequencies: before field application, 701 $\mathrm{kHz}, 746 \mathrm{kHz}$, and $796 \mathrm{kHz}$. At $701 \mathrm{kHz}$, the cells move towards the electrodes indicative of pDEP behavior and the intensity $<20 \mu \mathrm{m}$ and $>160 \mu \mathrm{m}$ increase above the background. The cells transition towards the center at $746 \mathrm{kHz}$, and focus by $796 \mathrm{kHz}$ indicative of $\mathrm{nDEP}$ behavior with the corresponding the peak intensity occuring around the center. This pDEP and nDEP behavior is similarly captured for all four profiles lines drawn (data not shown). 

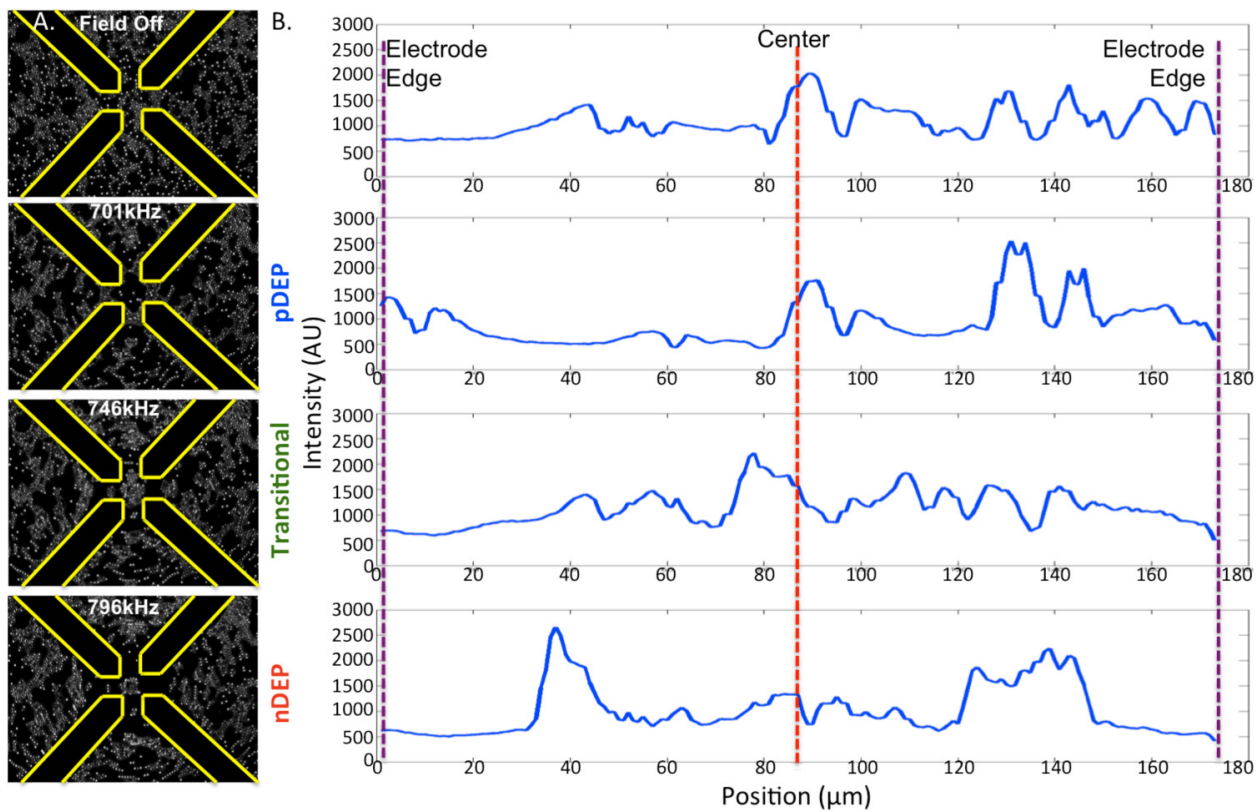

Figure 6.4. Video frames of $\mathrm{B}+$ cell behaviors in the dielectrophoretic microdevice at the noted frequencies (A) and the corresponding intensity profile oriented from top left electrode to bottom right electrode at the same frequency (B)

Stationary cells were neglected using the standard error threshold method described in section 6.3.5. Figure 30 demonstrates the intensity profiles obtained after filtering with standard error thresholds of $0.5,0.25$, and 0.1 . The bias curve is overlaid to demonstrate regions attributed to $\mathrm{nDEP}$ and those attributed to $\mathrm{pDEP}$. These four profile lines were biased with the curves in Figure 6.3B\&C, into DEP response predictions according to Equation (3) so that increased intensity in the center corresponded to nDEP behavior and increased intensity at the edges corresponded to strong pDEP behavior. The

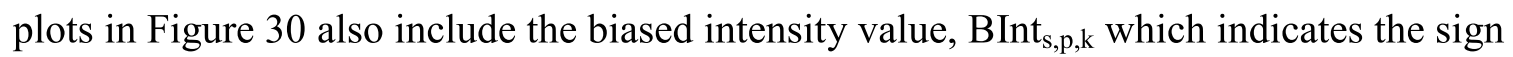
an strength of the DEP response predicted. At the standard error tolerance of 0.5 , consistently strong nDEP was recorded at all frequencies, while the video images reflect transitional behavior to $\mathrm{pDEP}$. Consistently $\mathrm{nDEP}$ responses are recorded for a tolerance of 0.25 , although the strength of this was much weaker. Only at a tolerance of 0.1 , was 
slightly positive DEP responses predicted at $424 \mathrm{kHz}$. Thus, further examination is needed to ascertain an optimal tolerance value to yield DEP responses that are consistent with the visually observed DEP phenomena.
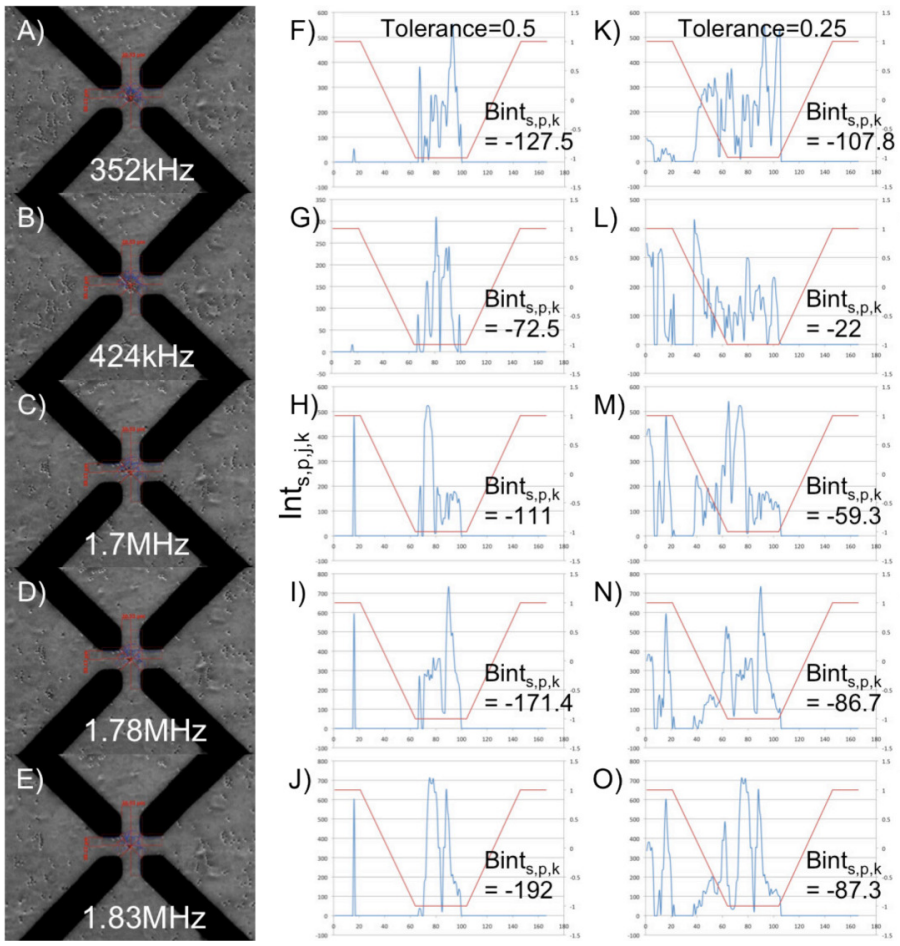

L)

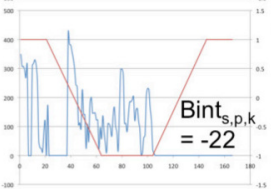

P) $=$ Tolerance $=0.1$
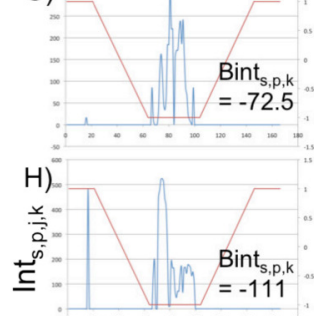

M)
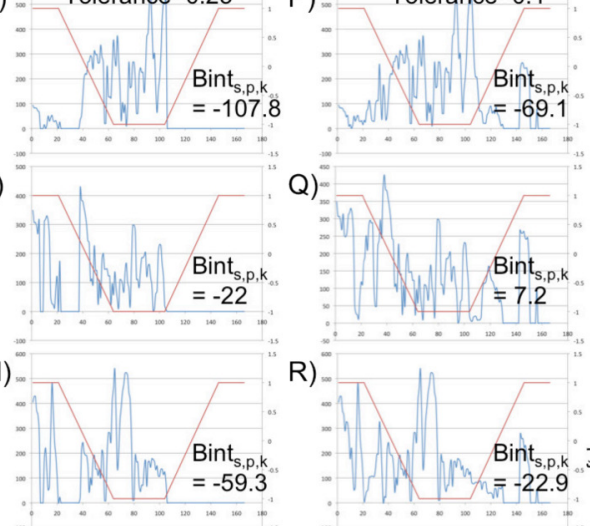

Q)

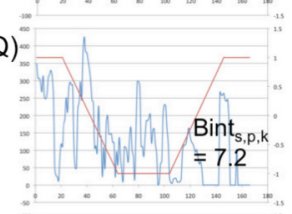

R)
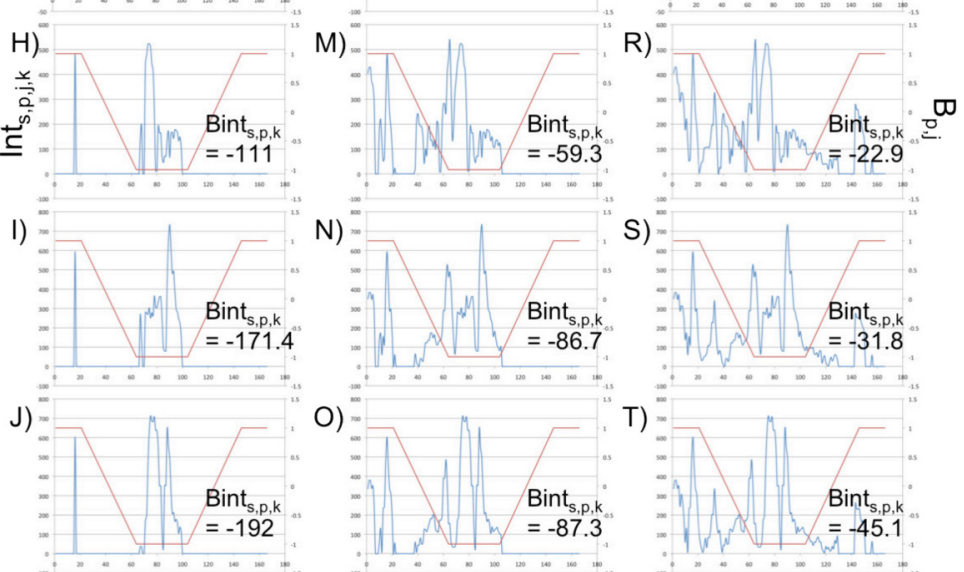

S)

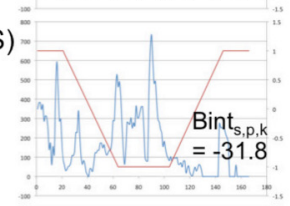

T)

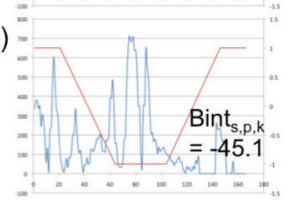

Figure 6.5. Video images of of native $O$ - sample in $0.1 \mathrm{~S} / \mathrm{m}$ medium during the $100 \mathrm{kHz}$ to $1.9 \mathrm{MHz}$ in $400 \mathrm{~s}$ sweep: $352 \mathrm{kHz}(\mathrm{A}), 424 \mathrm{kHz}(\mathrm{B}), 1.7 \mathrm{MHz}(\mathrm{C}), 1.78 \mathrm{MHz}(\mathrm{D})$ and $1.83 \mathrm{MHz}(\mathrm{E})$. Intensity absolute values (blue lines) for three different standard error tolerance levels: 0.5 (F-J), 0.25 (K-O) and 0.1 (P-T), each at the corresponding frequencies. The red lines are the upward diagonal bias curve. The biased intensity value from Equation (3) is noted on each plot.

The four profiles were thus transformed into biased intensities or DEP responses for each frequency and could then be plotted as a function of frequency to resemble DEP spectra.

Figure 6.6 presents the biased intensity as a function of frequency for each profile line.

The four profiles were combined as described into a combined DEP spectrum for the sweep according to Equation (5). Figure 6.6A, B, and C demonstrate the DEP spectra for standard error tolerances of $0.5,0.25$, and 0.1 . As with Figure 6.5 , strongly negative DEP 
behavior is predicted with the high standard error tolerance of 0.5 while transitional behavior is almost captured by the 0.1 level tolerance. The combined DEP response was obtained for each sweep. Comparisons of the sweeps are subsequently included in Figure 6.8. The sweeps were combined as described in Section 6.3.5 to determine a final DEP response versus frequency profile for each blood type at each solution condition. 


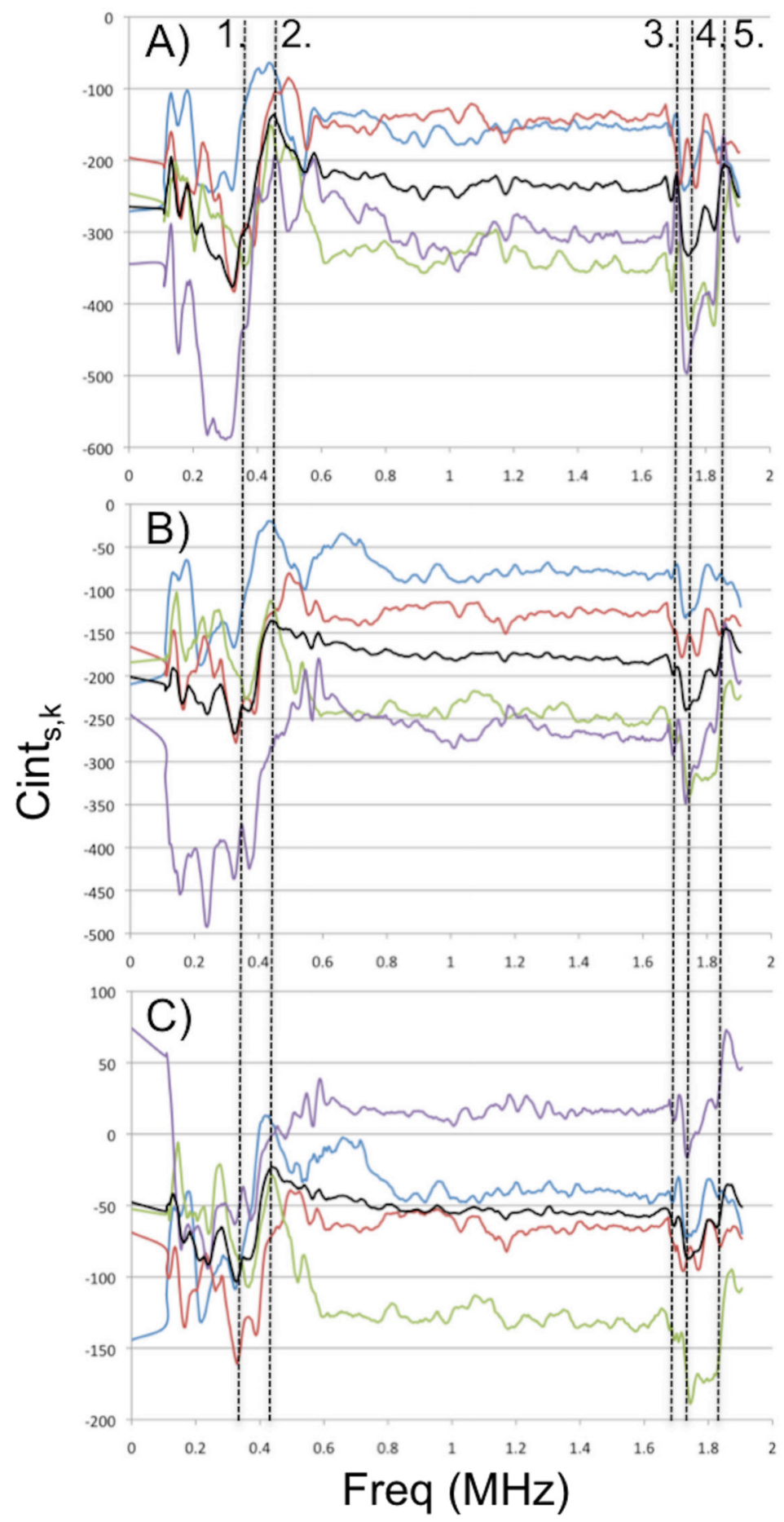

Figure 6.6. Biased intensity for all four profile lines: Downward (blue), Upward (red), Horizontal (green) and Vertical (purple), as well as the Combined Intensity (black) for the three standard error tolerance levels: 0.5 (A), 0.25 (B) and 0.1 (C). Dashed lines correspond to frequencies featured in Figure 6.5: 1. 352kHz, $2.424 \mathrm{kHz}, 3$. 1.7MHz, 4. 1.78MHz and 5. 1.83MHz. 
As mentioned above the cell DEP response curves were calculated by averaging the six sweeps into a weighted response. Cell DEP repsonses depend on medium conductivities because the frequency-dependent effective polarizability is heavily influenced by charge alignment on the surfaces of dielectric materials (i.e. cells). Therefore, the measured DEP repsonses for $\mathrm{B}+$ blood cells were compared for medium conditions of 0.01 and $0.1 \mathrm{~S} / \mathrm{m}$ as demonstrated in Figure 6.7A. Results show that the lower conductivities yielded more positive DEP behavior over the frequency range explored, which is roughly consistent with the theory in Chapter 3. ABO membrane antigens were also removed enzymatically to obtain modified cells and the DEP frequency response explored again for high and low medium conditions. Similarly, lower conductivities yielded more positive DEP behaviors. Comparisons of the native and modified cells at a given conductivity are shown in Figure 6.7C\&D. A shift to higher frequencies is observed for the transition from nDEP to pDEP at both the low and high medium conductivities. This result supports prior work that $\mathrm{ABO}$ expression influences the dielectric properties of erythrocytes manifesting in differing DEP frequency responses. 

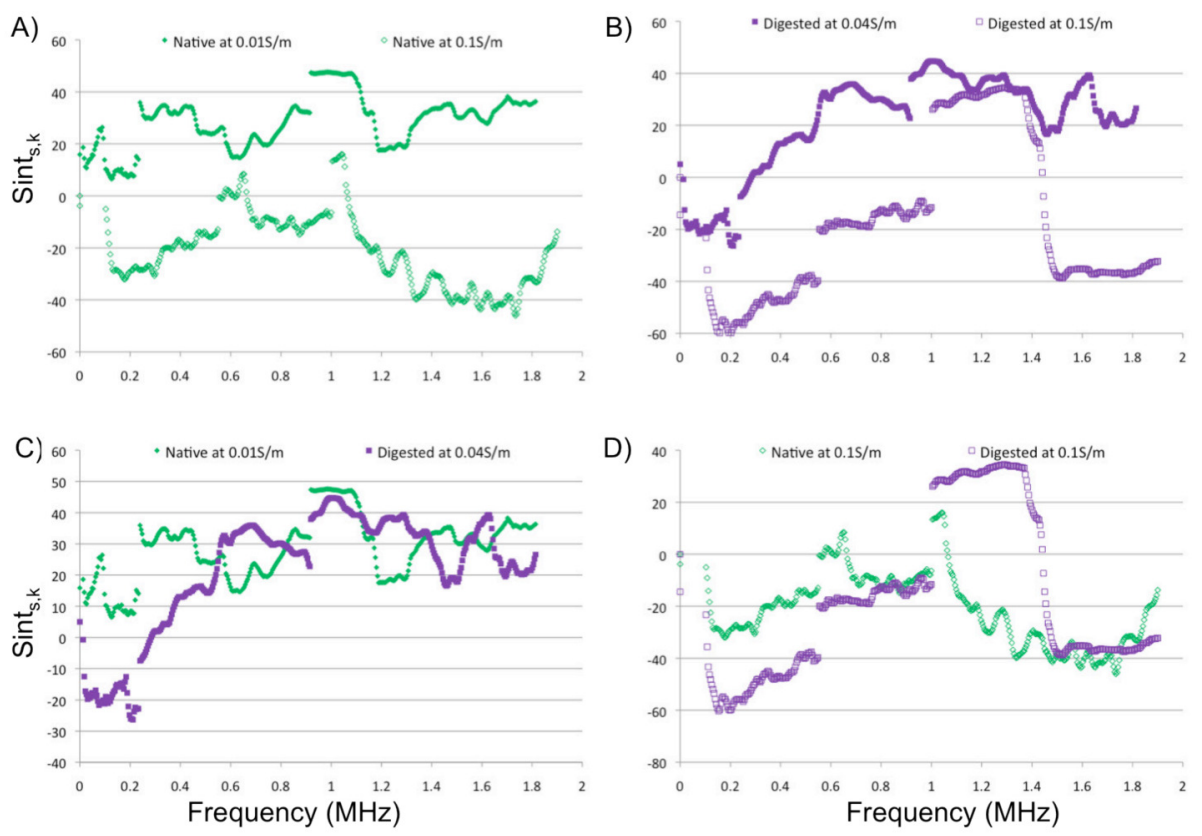

Figure 6.7. DEP response predictions for a $\mathrm{B}+$ donor. Green lines correspond to the native sample and purple the digested. Closed symbols are $0.01 \mathrm{~S} / \mathrm{m}$ medium conductivity while open symbols are $0.1 \mathrm{~S} / \mathrm{m}$ medium conductivity. Conductivity comparisons are in $A$ (native) and $B$ (modified) while native and modified are compared for the $0.01 \mathrm{~S} / \mathrm{m}(\mathrm{C})$ and $0.1 \mathrm{~S} / \mathrm{m}(\mathrm{D})$ medium conditions.

The final DEP frequency response curves for seven blood types are shown in

Figure 6.8. The first apparent general trend is that higher conductivity solutions (Figure 6.8C\&D) allow the blood cells to experience nDEP behavior over a longer frequency range than do the lower conductivity solutions (Figure 6.8A\&B). This is to be expected from the theory because when the medium is more polarizable than the cell, the cell will be pushed to the area of lowest field density so that the field lines can travel through the more conductive medium solution. Further, DEP repsonses were more consistent up to $\sim 1 \mathrm{MHz}$ in the higher conductivity solutions. At frequencies above $1 \mathrm{MHz}$, much larger differences exist between blood types. The cell's effective polarizability is heavily influenced by the conductivity and frequency applied quotient, so at higher frequencies, conductivity effects become less dominant and the effective polarizability is more heavily influenced by permittivity. The transition to $\mathrm{pDEP}$ and for many blood types back to 
$\mathrm{nDEP}$ is a result of the coplex interaction of the permittivity of the cell membrane or shell in the models.

Another apparent trend from this presentation of the data is that less spread exists between the DEP spectra after modification than with the native DEP spectra. This follows the previous results which found that the $\mathrm{COF}$ range decreased from $17 \mathrm{MHz}$ to $5 \mathrm{MHz}$ post-modification ${ }^{1}$. The implications of this result are that $\mathrm{ABO}$ antigen removal clearly manifests in an altered DEP response and this response is closer to that of a baseline erythrocyte. However, variations still exist which suggest other antigens or phenotypic expression influence the dielectric properties of the cell and thus the DEP frequency responses observed.
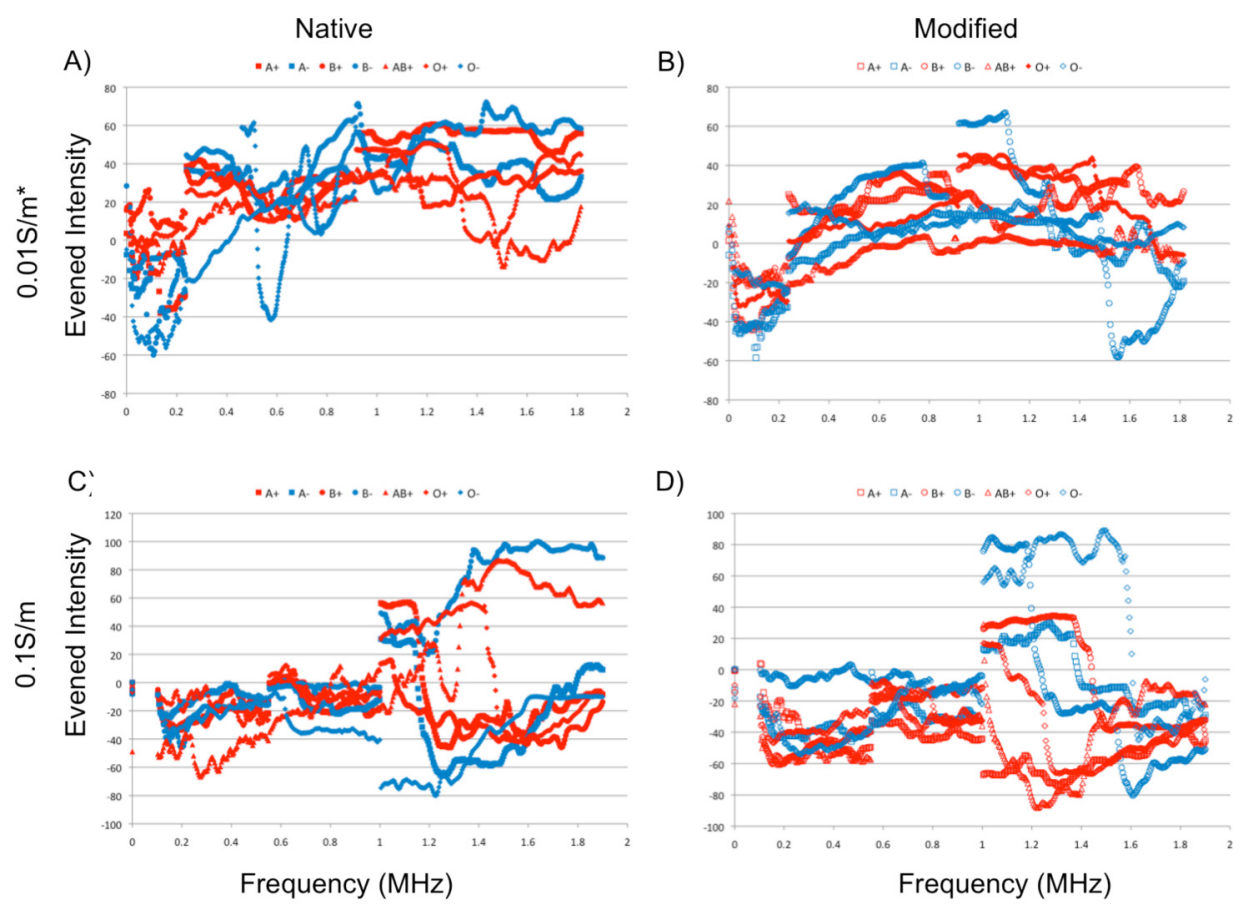

Figure 6.8. Experimentally predicted DEP response for a given blood type at two medium conditions and two cell treatement conditions: native at $0.01 \mathrm{~S} / \mathrm{m}(\mathrm{A})$, modified at $0.04 \mathrm{~S} / \mathrm{m}(\mathrm{B})$, native at $0.1 \mathrm{~S} / \mathrm{m}(\mathrm{C})$, and modified at $0.1 \mathrm{~S} / \mathrm{m}$ (D). The positive blood types are in red and the negative blood types are in blue. Native cells are filled symbols and the modified cells have open symbols. 
Four unique donors were recruited for each blood type. Figure 6.8 includes data for the average across all donors that were currently available for that $\mathrm{ABO}-\mathrm{Rh}$ blood type. Further analysis including a collaboration with a Michigan Tech statistician is ongoing. For the native blood cells at low conductivity in Figure 6.8A, the general trend is that the cells experience $\mathrm{nDEP}$ or slight $\mathrm{pDEP}$ behavior at low frequencies and slowly increase to a maximum pDEP behavior. It is also difficult to distinguish between any of the blood types under this condition because with the exception of $\mathrm{O}+$ and $\mathrm{AB}+$, which dip down in the negative DEP region briefly, they all follow the same upward pDEP trend and the response curves are too noisy to discern differences in cross over frequency. For the native blood cells suspended in the higher conductivity buffer of $0.1 \mathrm{~S} / \mathrm{m}$ Figure 6.8C, negative DEP is observed until $\sim 1 \mathrm{MHz}$. At frequencies above $1 \mathrm{MHz}$, DEP behaviors of blood types differ. $\mathrm{O}+$ experiences stronger $\mathrm{pDEP}$ at lower frequencies than all the other blood types and B- experiences the strongest pDEP behavior around 1.81.9MHz. A+ remains in the pDEP region above $1 \mathrm{MHz}$, while the other blood types transition back to $\mathrm{nDEP}$.

To examine Rhesus factor interactions, the graphs in Figure 6.8 were plotted in pairs to compare the same $\mathrm{ABO}$ antigen, but differing $\mathrm{Rh}$ expression (i.e. $\mathrm{A}+\mathrm{can}$ be compared to A-, etc.). At $0.1 \mathrm{~S} / \mathrm{m}$ and $0.01 \mathrm{~S} / \mathrm{m}$ conductivities, the native blood cells demonstrate similar DEP curves with frequency, especially for A+ compared to A-. This behavior would indicate that the Rhesus factor has a minor or negligible influence low frequency DEP behavior. The same conclusion was obtained in Chapter 5, where results indicated that the $\mathrm{ABO}$ antigens impacted the low $\mathrm{COF}$ values $30.5 \mathrm{MHz}$ (A-) to 47.5 
$\mathrm{MHz}(\mathrm{O}-)$ and the Rhesus factor expression impacted the high COF value $64.5 \mathrm{MHz}(\mathrm{A}-)$ to greater than $80 \mathrm{MHz}(\mathrm{O}-)$.

Blood cells were modified to remove $\mathrm{ABO}$ antigens without altering the $\mathrm{Rh}$ factor. For the modified cells suspended in the high conductivity medium, A+ differs greatly from $\mathrm{A}-\mathrm{A}+$ displays nDEP behavior from 0.01 to $1.9 \mathrm{MHz}$ while $\mathrm{A}$ - experiences a range of $\mathrm{pDEP}$ behavior from approximately $1 \mathrm{MHz}$ to $1.4 \mathrm{MHz}$. $\mathrm{B}+$ and $\mathrm{B}-$ display similar behaviors except the pDEP to nDEP transition around $1.22 \mathrm{MHz}$ is lower for $\mathrm{B}$ - than for $\mathrm{B}+(1.44 \mathrm{MHz})$. Behaviors are similar between $\mathrm{O}+$ and $\mathrm{O}-$ with an $\mathrm{nDEP}$ to $\mathrm{pDEP}$ transition at a similar frequency of $1 \mathrm{MHz}$, but the pDEP to $\mathrm{nDEP}$ transition occurs at a much lower frequency for $\mathrm{O}+(1.1 \mathrm{MHz})$ than $\mathrm{O}-(1.6 \mathrm{MHz})$. Overall, these comparisons suggest variations due to genetic diversity possibly from other blood group antigen expressions, but since there is not a consistent trend with Rhesus factor expression, it is not possible to conclude an Rh dependence on the DEP behaviors observed.

The ability to sweep the frequency and observe DEP reponses was a major advancement over the techniques presented in Chapter 5 in which a frequency was fixed and the DEP response measured. However, as Figure 6.9 demonstrates, the sweep rate potentially skewed the DEP behaviors observed and thus captured by the intensity profile analysis. Since this is not a desired experimental result, the frequency sweeps conducted were modified to systematically test this dependency. The sweep rates run were modified to Table 6.3 and include at least one $2 \mathrm{x}, 1 \mathrm{x}, 0.5 \mathrm{x}$ and $0.25 \mathrm{x}$ sweep rate for each blood sample. Image comparisons at 500kHz in Figure 6.9A demonstrate differing observed DEP responses, which are reflected in the differing DEP response curves in Figure 6.9B. The equipment (AC generator) available was limited to a maximum sweep duration of 
500 seconds. In the experiments presented, the fast sweep at $0.0045 \mathrm{MHz} / \mathrm{sec}$ from $100 \mathrm{kHz}$ to $1.9 \mathrm{MHz}$ in $400 \mathrm{~s}$ tended to miss the initial nDEP behavior. This behavior was observable at slower sweep rates $100 \mathrm{kHz}$ to $550 \mathrm{kHz}$ over $200 \mathrm{~s}$ sweep and is consistent with dielectrophoretic theory in this range. Further, Figure $6.9 \mathrm{C}$ shows that a sweep rate of $1 \mathrm{x}$ yield inconsistent DEP spectra between repeated runs. However, the $0.25 \mathrm{x}$ and $0.5 \mathrm{x}$ runs are relatively consistent over the narrow frequency range examined. This will be systematically explored to field optimal conditions for all subsequent experiments.

Table 6.3.

Sweeps used to test for rate dependency. Each sample was run for at least one $2 \mathrm{x}, 0.25 \mathrm{x}$, and $0.5 \mathrm{x}$ sweep.

\begin{tabular}{l|lllll}
\hline \multicolumn{1}{c}{ Solution } & $\begin{array}{c}\text { Start Frequency } \\
(\mathbf{M H z})\end{array}$ & \multicolumn{1}{c}{$\begin{array}{c}\text { End Frequency } \\
(\mathbf{M H z})\end{array}$} & \multicolumn{1}{c}{ Time (sec) } & \multicolumn{1}{c}{ Rate } \\
\cline { 2 - 6 } & 0.01 & 0.91 & 200 & $2 \mathrm{x}$ \\
$\mathbf{0 . 0 1 S / m}$ with & 0.01 & 1.81 & 400 & $2 \mathrm{x}$ \\
$\mathbf{0 . 7 5 \%}$ BSA & 0.01 & 0.460 & 400 & $0.5 \mathrm{x}$ \\
and & 0.01 & 0.460 & 200 & $1 \mathrm{x}$ \\
$\mathbf{0 . 0 4 S} / \mathbf{m}$ with & 1.81 & 400 & $2 \mathrm{x}$ \\
$\mathbf{1 . 5 \%}$ BSA & 0.01 & 0.29125 & 500 & $0.25 \mathrm{x}$ \\
& 0.01 & 1 & 200 & $2 \mathrm{x}$ \\
\hline $\mathbf{0 . 1 S} / \mathbf{m}$ & 0.1 & 1 & 400 & $1 \mathrm{x}$ \\
$\mathbf{a n d}$ & 0.1 & 1.9 & 400 & $2 \mathrm{x}$ \\
$\mathbf{0 . 1 S} / \mathbf{m}$ with & 0.1 & 1 & 400 & $1 \mathrm{x}$ \\
$\mathbf{1 . 5 \%}$ BSA & 0.1 & 0.6625 & 500 & $0.5 \mathrm{x}$ \\
& 0.1 & 0.381 & 500 & $0.25 \mathrm{x}$ \\
\hline
\end{tabular}

Based on the data analyzed to date, the recommendation is to conduct longer duration sweeps that run over a short frequency range so that the rate of $\mathrm{MHz}$ per second is less than or equal to $0.001125 \mathrm{MHz} / \mathrm{sec}$ (designated at $0.5 \mathrm{x}$ in Figure 6.9). This slower frequency shift allows the erythrocyte to experience each frequency for a longer duration and thus minimizing charge migration and polarization lag phenomena to achieve an effective polarizability closer to steady state. In summary, it is recommended for further 
experiments that sweep rate is less than or equal to $0.001125 \mathrm{MHz} / \mathrm{sec}$ (corresponds to $0.5 \mathrm{x})$ 


\section{$500 \mathrm{kHz}$}
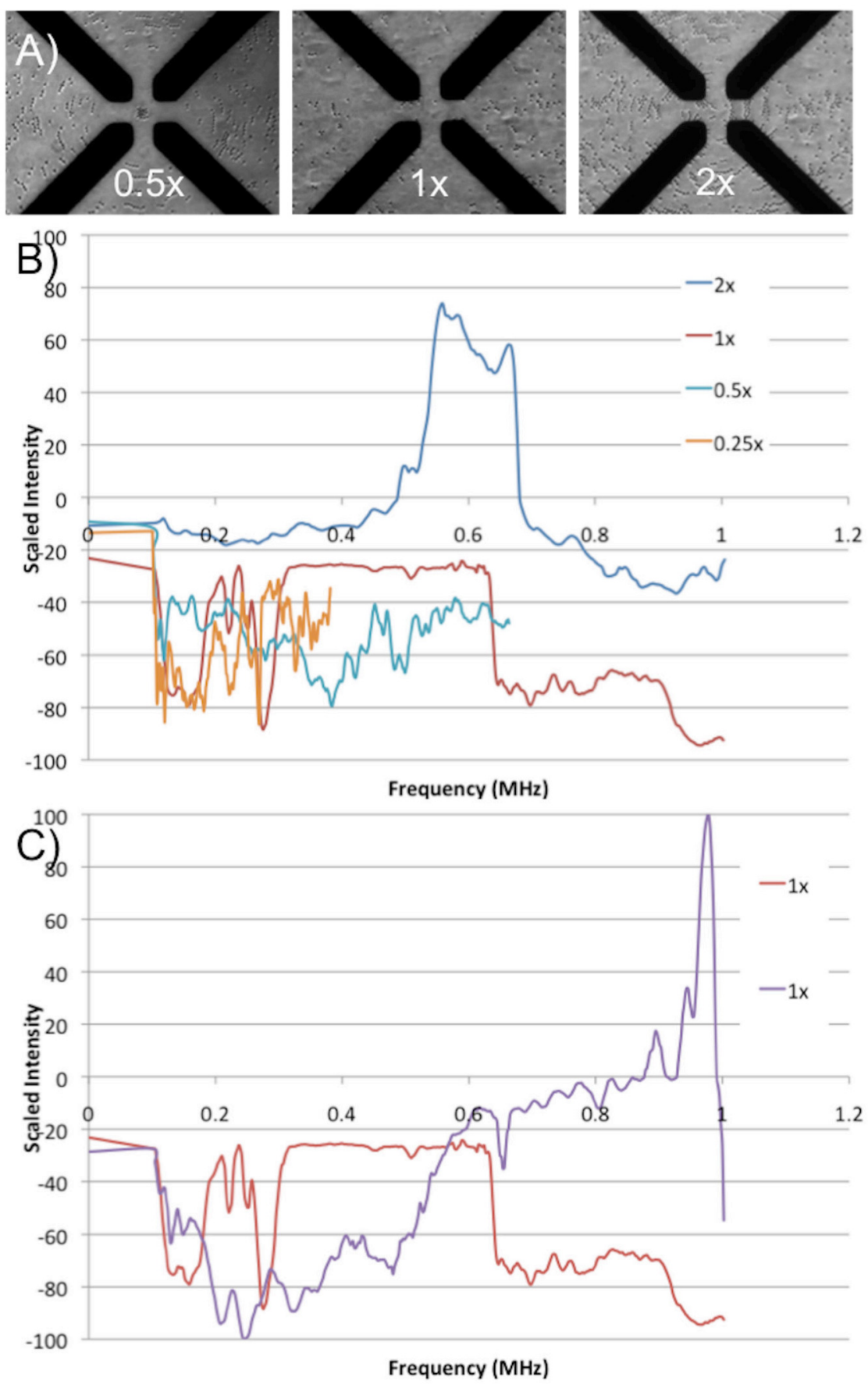

Figure 6.9. Video images (A) of native 0 - blood in $0.1 \mathrm{~S} / \mathrm{m}$ medium at $500 \mathrm{kHz}$ for $0.5 x$, $1 \mathrm{x}$ and $2 \mathrm{x}$ sweep. DEP Response curves $(B)$ for $0.25 x, 0.5 x, 1 x$, and $2 x$ sweep rates. The $0.25 x$ sweep is noisy with limited frequency range and the $2 x$ sweep is so rapid that the cells experience pDEP when no other sweep registers this behavior. DEP response curves for two different $1 \mathrm{x}$ sweeps $(100 \mathrm{kHz}$ to $1.9 \mathrm{MHz}$ in $400 \mathrm{~s})$ which demonstrates inconsistent behavior between runs. Slowe sweep rates yield more consistent behaviors. 
In addition to completing standard error threshold corrections and the statistical analysis of donors, the correlation of the experimental results with the theory presented in Chapter 3 will be completed. The first attempt at correlating these two will be to use a least-squares analysis to determine more accurately the four parameters influencing the Clausius Mossottie factor and thus the calculation of the dielectrophoretic force on a cell: cytosolic and membrane permittivity and conductivity. A Matlab code is in development to itteratively change the 4 parameters in order to minimize the distance between the theory curve and the quantified DEP experimental behavior. As discussed in Chapter 3, future work should adjust these dielectric parameters by including a second shell around the particle tailorable to account for the $\mathrm{ABO}$ antigen expression.

\subsection{Conclusions}

Building upon previous work, which explored the DEP behavior of human erythrocytes suspended in a medium of conductivity $0.9 \mathrm{~S} / \mathrm{m}^{1}$, the DEP behavior of erythrocytes in medium of $0.01 \mathrm{~S} / \mathrm{m}$ and $0.1 \mathrm{~S} / \mathrm{m}$ was explored in order to compare to other cell literature $3,4,8,140,162,164,167,226,227,233$. In addition to this medium conductivity dependency, the human erythrocytes were modified with $\beta$-galactosidase to explore ABO antigen influences on DEP behavior. Both the native and the modified samples were tested at $0.01 \mathrm{~S} / \mathrm{m}$ and $0.1 \mathrm{~S} / \mathrm{m}$, combining to four treatment combinations.

Experimental images from six different sweeps rates/ranges were obtained for all four treatment combinations. A Matlab code was written to manipulate the intensity data from profile lines drawn in the Zeiss software and correlated these to position in order to obtain a DEP response profile as a function of frequency. Through a series of mathematical manipulations described in the materials and methods, these individual 
profiles were combined for a given sweep and then combined across sweeps for each treatment type.

It was found that the intensity profiles, which were modified and combined to mimic typical dielectrophoretic curves, do vary between blood types. The blood types demonstrated cross over frequency behaviors (if observed) at different frequencies. Modifications to the standard error threshold are necessary to ensure robustness of the data. However, it was also found that the sweep rate and the sweep starting and ending parameters affected the observed behavior. For the fastest sweeps, for example low conductivity going from $10 \mathrm{kHz}$ to $910 \mathrm{kHz}$ in 200 seconds (1x), the initial nDEP behavior was often missed but then was caught by a slower sweep like the one going from $10 \mathrm{kHz}$ to $460 \mathrm{kHz}$ in 400 seconds $(0.5 \mathrm{x})$. For future experiments it is recommended that the sweeps used cover $450 \mathrm{kHz}$ over 400 seconds in order to insure that behavior is not skipped. 


\subsection{Acknowledgements}

All work was performed in an Institutional Biosafety Committee (IBC) certified

Biosafety Level 2 laboratory and received approval from the Internal Review Board (IRB) at Michigan Technological University for the protection of human subjects (Approval \#M0540). Work was supported by the NSF CBET CAREER

(0633538/1041338) and NSF GRS $(0737864,0841403,0942772)$. The authors have declared no conflict of interest. 


\section{Conclusions}

The work presented herein has shown that human erythrocytes can and should be used for on-chip cellular analysis with alternating current dielectrophoresis. This document has presented the current state of literature in regards to both the human blood system and dielectrophoretic theory and experimentation. It has also presented experimental results regarding low $\mathrm{kHZ}$ erythrocyte rupturing, $\mathrm{ABO}$ antigen modification with $\beta(1-3)$ galactosidase and DEP of human erythrocytes as a function of medium conductivity, frequency and blood type. These results show that the polarizability of a human erythrocyte is highly dependent on its antigen expression, specifically its ABO-Rh antigen expression. It has shown that while this is not currently looked at as a factor in DEP studies that it should be noted because the baseline responses are not equal for all eight blood types $(\mathrm{A}+, \mathrm{A}-, \mathrm{B}+, \mathrm{B}-, \mathrm{AB}+, \mathrm{AB}-, \mathrm{O}+$ and $\mathrm{O}-)$ and therefore any deviations from the baseline due to disease also cannot be expected to be equal. This work has shown that molecular level differences in the cell can be detected using dielectrophoresis in conjunction with microdevices.

\subsection{Current State of Literature}

The human blood system is comprised of erythrocytes, leukocytes, platelets and the suspending plasma. All three cellular components are derived from the same hematopoietic stem cell but after differentiation have different sizes, shapes and functions. The fields of biochemistry and microbiology have yielded insights into cellular function, 
genotype/phenotype composition, and morphology. The field of electrokinetics has advance to determine the dielectric properties of various blood cell types. However, these two research approaches remain disconnected, which hinders the knowledge that can be gained about blood cell function, phenotype, and morphology.

A great deal of work has been done to explore the dielectrophoretic behavior of particles $8,17,157,158,160,164,166,167,175,183-186$ and cells $1,3-9,12-14,118,123,124,158,159,161,162,188-192$. In the majority of these works the experimental results have been tied to the theory presented above so that more is learned regarding the dielectric parameters of various particle and cell types. These fields have the opportunity to connect dielectric properties to biochemical or biophysical properties of the cell. A few notable groups have begun to accomplish this including extensive work on differentiating healthy and $P$. falciparum infected erythrocytes ${ }^{4,5}$ and exploring the molecular-level changes between the same erythrocyte types ${ }^{1,10,11,145-148,151-156}$. Much progress remains to be done to closely couple the biochemical phenotype to the dielectric properties.

The work presented herein is aimed at furthering the field's understanding of how molecular level changes can appreciably affect dielectrophoretic behavior and dielctric constants. This work adds another layer of affects that need to be considered whenever blood is the diagnostic fluid of use in a dielectrophoretic microdevice. The blood type of the human erythrocyte must be considered from an experimental standpoint and an additional factor needs to be included in the governing equations for dielectrophoretic theory. This work has opened up a subfield of cellular dielectrophoretic research where parameters and affects that were hence considered not to affect the normal behavior now are found to have an impact. Because the ABO-Rh antigens affect 
dielectrophoretic behavior it is possible that other surface and transmembrane antigens could affect $\mathrm{nDEP}$ and $\mathrm{pDEP}$ responses of that cell.

\subsection{Low kHz Erythrocyte Rupturing}

Erythrocyte rupture in a $1 \mathrm{kHz}$ dielectrophoretic field was quantified to determine the viability of using AC DEP fields for erythrocyte rupture and to discern any dependencies on rupturing kinetics of ABO-Rh blood cells. As early as 5 minutes into the experiment it is possible to distinguish $\mathrm{B}+$ from $\mathrm{AB}-, \mathrm{O}+$ and $\mathrm{O}-$ at a $95 \% \mathrm{CI}$ as indicated by * in Figure 4.2D. By the time 10 minutes passes, it is also possible to distinguish $\mathrm{A}+$ and $\mathrm{A}$ - from $\mathrm{AB}-, \mathrm{O}+$ and $\mathrm{O}-$ at a 95\% CI. Thus, $\mathrm{ABO}$ and Rhesus factor antigens alter the cell's response to an electric stimulus, suggesting the Maxwell-Wagner membrane polarization phenomenon is molecule dependent. Further, this ion/molecule/membrane interaction manifests in different blood types exhibiting different rupturing kinetics. A first order exponential decay was fit to $\mathrm{A}+, \mathrm{A}-$ and $\mathrm{B}+$ time dependent rupturing fraction, with kinetic rate constants of: $\mathrm{A}+\left(0.02 \pm 0.002 \mathrm{sec}^{-1}\right)$ and $\mathrm{B}+\left(0.11 \pm 0.008 \mathrm{sec}^{-1}\right)$ and $\mathrm{A}-\left(0.04 \pm 0.04 \mathrm{sec}^{-1}\right)$.

Experiments reveal from 0.002 to 0.282 variation within a single donor tested 5 days apart suggesting that the variation between donation days was not significant. Insignificant variations on the order of 0.001 to 0.1 were observed between microdevices when using the same blood sample. Even though the observations were not necessarily always significant at a $95 \%$ CI there has been previous research done on the aging effects in the form of chemical and morphological surface of the platinum wires ${ }^{225}$ that can alter and reduce reliability of dielectrophoretic quantification. 
Additionally dependencies were explored in order to direct future experiments towards the parameters, which would lead to the best rupturing conditions. Blood aging effects were tested by performing tests on the same blood sample on the day of donation and every two days thereafter and the change with blood age was found to only be significant at a $95 \% \mathrm{CI}$ for $\mathrm{O}$ - according to Table 4.5. It was determined via a two-factor ANOVA without replication for the electric field strength dependency that the resulting $\mathrm{F}$ value of 7.66 is greater than the $F_{\text {critical }}$ value of 5.14 so for all blood types there is a statistically significant difference in overall rupturing fraction at an alpha level of 0.05 . This implies that generally, rupturing fraction will increases as electrical field strength increases. For both $\mathrm{B}+$ and $\mathrm{O}-$ the two-factor ANOVA with replication showed that the null hypothesis that there was no discernable difference in mean overall rupturing fraction with respect to concentration could not be rejected. This result is largely positive because it indicates that it is likely that the whole blood sample could be used on-chip and the fraction of cells ruptured over a given time period would stay consistent for a blood type regardless of the starting hematocrit level of the sample. The most telling results are those that tie directly into the larger body of literature exploring the dielectrophoretic behavior of cells as a function of frequency ${ }^{1,3-9,12-}$ $14,118,123,124,158,159,161,162,188-192$. A single donation each was tested for 50 minutes at frequencies ranging from $1-10 \mathrm{kHz}$ for $\mathrm{A}^{+}, \mathrm{A}^{-}, \mathrm{AB}^{+}, \mathrm{AB}-, \mathrm{O}^{+}$and $\mathrm{O}-$ and as seen in Figure 4.5: $\mathrm{A}+$ experiences its maximum fraction of cells ruptured at $4 \mathrm{kHz}, \mathrm{A}-$ at $6 \mathrm{kHz}$, $\mathrm{AB}+$ at $1 \mathrm{kHz}, \mathrm{AB}-$ at $6 \mathrm{kHz}, \mathrm{O}+$ at $2 \mathrm{kHz}$ and $\mathrm{O}-$ at $3 \mathrm{kHz}$. These results indicate that both the $\mathrm{ABO}$ antigens and Rhesus factor affect rupturing. This dependence on frequency could be extremely useful if further explored because if a blood type of a given sample is 
known it would likely be possible to control the amount of rupture based on both exposure time and frequency.

This work has proven that it is possible to rupture human erythrocytes using the alternating current dielectrophoretic force. This provides a low-cost, chemical-free and relatively safe way of rupturing cells that does not require highly technical fabrication techniques. Due to these factors, rupturing with AC DEP has advantages over chemical, mechanical and electroporation-induced lysis. We have also shown that not only is it possible to rupture some human erythrocytes with AC DEP, and that this rupture is dependent on the ABO-Rh blood type of the sample. This opens this technique up to possible uses ranging from subcellular analysis to blood type determination to therapeutic techniques. As of now, it is possible to differentiate $\mathrm{A}+, \mathrm{A}-$ and $\mathrm{B}+$ from the other blood types tested at $95 \%$ CI because they experience significant rupturing and can be fit to a kinetic decay rate. Further experiments are needed to tease out the exact kinetic rates of the other blood types and to look at medically significant confidence intervals of closer to 99\%. Future work will also include micropatterned electrodes instead of wire to ease fabrication and decrease any experimental error due to device differences. This work has shown that it is possible to rupture human erythrocytes, accurately predict the overall fraction of cells ruptured for A+ and B-, to identify blood type by their rupturing kinetics and use a variety of experimental parameters to further enhance the desired rupturing fraction. 


\subsection{Antigen Modification}

This work demonstrated that modification of human erythrocytes with $\beta(1-3)$ galactosidase is possible and was verified via HPLC, UV-Vis and agglutination testing . This modification also caused an appreciable change in the lower cross-over frequency during those experiments performed in a $0.9 \mathrm{~S} / \mathrm{m}$ medium conductivity. The range of low COF values dropped from $17 \mathrm{MHz}$ to $5 \mathrm{MHz}$ post-modification and since the $\beta(1-3)$ galactosidase only reacted with $\mathrm{ABO}$ antigens and would not have altered the $\mathrm{Rh}$ antigens, this result indicated that the lower COF is dependent on membrane surface expression. The higher COF value only decreased by $2 \mathrm{MHz}$, indicating that this behavior is likely controlled by the Rhesus factor not the $\mathrm{ABO}$ antigens ${ }^{1}$.

The implications of this work are two-fold: it proved that it is possible to cleave off the characteristic $\mathrm{ABO}$ antigens and it provided the strongest evidence that the dielectrophoretic behavior of the human erythrocytes is $\mathrm{ABO}$ type dependent. The work initially published using modification of human erythrocytes with $\beta(1-3)$ galactosidase $^{1}$ was, to the best of the author's knowledge, the first work to show that cleavage of the $\mathrm{ABO}$ antigen was possible using this enzyme. This has the potential to advance research into developing a uniform blood type for emergency transfusions when the blood type of a patient is unknown. Secondly, because the range of COF values decreased so dramatically after modification, there is now strong evidence that the $\mathrm{ABO}$ antigens at least in part control the DEP response of the human erythrocytes. Not only did the blood types exhibit a different lower COF than their unmodified counterparts, but the resulting COF from all blood types was within a $5 \mathrm{MHz}$ range of each other indicating that the 
erythrocytes reached a state where they began to experience the same or similar polarizability.

\subsection{DEP of Human Erythrocytes}

Cross-over frequencies observed in initial DEP experiments performed at $0.9 \mathrm{~S} / \mathrm{m}$ experiments differed from literature values ${ }^{4,123}$ most likely because our $0.9 \mathrm{~S} / \mathrm{m}$ medium conductivities were an order of magnitude larger. Theory and experimental results

support that cross-over frequency is a strong function of medium conductivity ${ }^{8,12,242}$ and that DEP force and thus phenomenological (positive/negative) motion is accentuated at lower medium conductivities. However, performing DEP at physiological conditions close to pure blood plasma $(\sim 1.1 \mathrm{~S} / \mathrm{m})$ provides an advantage in medical microdevice technology because it might be possible to perform DEP diagnostics with minimal dilutions or in vivo.

Building upon previous work, which explored the DEP behavior of human erythrocytes suspended in a medium of conductivity $0.9 \mathrm{~S} / \mathrm{m}^{1}$, the DEP behavior of erythrocytes in medium of $0.01 \mathrm{~S} / \mathrm{m}$ and $0.1 \mathrm{~S} / \mathrm{m}$ was explored in order to compare to other cell literature $3,4,8,140,162,164,167,226,227,233$. In addition to this medium conductivity dependency, the human erythrocytes were modified with $\beta$-galactosidase to explore ABO antigen influences on DEP behavior. Both the native and the modified samples were tested at $0.01 \mathrm{~S} / \mathrm{m}$ and $0.1 \mathrm{~S} / \mathrm{m}$, combining to four treatment combinations. A Matlab code was written to manipulate the intensity data from profile lines drawn in the Zeiss software for the six sweeps run for each treatment condition and correlated these to position in order to obtain a DEP response profile as a function of frequency. Through a series of 
mathematical manipulations described in the materials and methods of Chapter 6 , these individual profiles were combined for a given sweep and then combined across sweeps for each treatment type.

It was found that the intensity profiles, which were modified and combined to mimic typical dielectrophoretic curves, do vary between blood types. However, it was also found that the sweep rate and the sweep starting and ending parameters affected the observed behavior. For the fastest sweeps, for example low conductivity going from $10 \mathrm{kHz}$ to $910 \mathrm{kHz}$ in 200 seconds $(1 \mathrm{x})$, the initial nDEP behavior was often missed but then was caught by a slower sweep like the one going from $10 \mathrm{kHz}$ to $460 \mathrm{kHz}$ in 400 seconds $(0.5 \mathrm{x})$. For future experiments it is recommended that the sweeps used cover $450 \mathrm{kHz}$ over 400 seconds in order to insure that behavior is not skipped. Also, minor modifications are needed to the Matlab code to more accurately represent the tolerance for non-moving cells.

In summary, the $\mathrm{ABO}$ antigen structures and chemical compositions play a key role in an erythrocyte's effective polarizability in dielectrophoretic fields. Since ABO antigens are expressed on the membrane surface, they likely impact surface conductivity; whereas the transmembrane Rh-D antigen likely influences the permittivity of the membrane. Thus, A, B or $\mathrm{O}$ antigens affects the dielectric properties of the cell membrane and therefore the dielectrophoretic force on the entire cell at a specified frequency.

\subsection{Future Work}

The work presented thus far shows great promise in being able to distinguish between ABO-Rh blood types based on the erythrocytes response to a dielectrophoretic 
force. It has been shown that $\mathrm{ABO}-\mathrm{Rh}$ antigens appreciably change dielectrophoretic behavior of cells in the manners of rupture, $\mathrm{nDEP}, \mathrm{pDEP}$ and COF values. The biggest problem faced in this research was large confidence intervals in most of the projects. With a larger donor population these confidence intervals could be decreased and the results could reach medical significance levels. It is the recommendation of this author that this work be continued to further explore the affect of the ABO-Rh antigens on dielectrophoretic behavior of human erythrocytes by increasing the number of donors per blood type. Due to the availability of willing donors with proof of blood type this would likely mean partnering with a hospital is necessary but the results gleaned should be well worth the effort of the partnership.

After highly reproducible dielectrophoretic curves are found for each of the ABORh blood types, the next logical step in research projects is to begin to discern differences between a healthy human erythrocyte of known blood type and an unhealthy erythrocyte of the same type. Since such a body of work already exists regarding cancerous cells, it would be convenient to start with testing leukemia cells and see if these exhibit a different dielectrophoretic signature than the health human erythrocytes.

The author also thinks that a medical microdevice could be fabricated to make use of the rupturing kinetics observed in this project. The rupture research was conducted with platinum wire electrodes so it first needs to be replicated with copper/gold plated electrodes in order to decrease variability between samples. Once reproducibility is achieved, the rupturing of human erythrocytes with DEP will prove to be a useful mechanism in medical microdevices because it can be easily integrated with subcellular analysis. For example, there has been some initial work done in the author's group to 
determine resistance differences between different hemoglobin phenotypes to explore the DEP of sickle cell anemia. A potential microdevice could take the rupture work presented in this dissertation and couple that with the exploration of the resistance of a protein such as hemoglobin to gather additional information about the biochemical and biophysical state of the erythrocyte. As mentioned above, utilizing microdevices as groups of building blocks is gaining interest ${ }^{126}$, and such a device would easily make use of the low-cost, no chemical and low field strength DEP rupturing mechanism presented in this work.

The final step in the additional work that needs to be completed for this project is the direct comparison of reproducible experimental results to the current thin shell ellipsoidal model. This must be done to determine what exactly are the differences between the dielectric parameters of human erythrocytes based on their differing antigen expression and to determine if these antigen dependencies can be included in the theory. It seems likely that the ABO antigens, which are surface antigens, could be added onto the current core-shell model as yet another shell outside the membrane. The dielectric properties (permittivity and conductivity) of this shell would therefore depend on whether or not the cell expressed $\mathrm{A}$ and $\mathrm{B}$ antigens or if it expressed only the $\mathrm{O}$ antigen. These exact parameters could be teased out experimentally using the same $\beta(1-3)$ galactosidase modification that has been presented in this work because the modification would bring all cells to the same outer shell type and then as A and B antigens are added, the outer shell parameters would change.

Overall, this work has provided the much needed answer to whether or not molecular level differences can be detected using dielectrophoresis and if they can, is 
antigenic expression strong enough that this could be one of the differences detected. The overall HYPOTHESIS of this project was that structural differences in the ABO-Rh antigens on or in the membrane cause a difference in the human erythrocyte's response to an alternating current dielectrophoretic field. This work strived to statistically differentiate between blood types using a small panel of 4 donors for each of the $8 \mathrm{ABO}-\mathrm{Rh}$ blood types. This hypothesis was proven to be likely via three different experimental sections: rupturing of human erythrocytes (Chapter 4), high medium conductivity COF analysis of human erythrocytes (Chapter 5) and lower medium conductivity sweeps of human erythrocytes (Chapter 6). With more donors and greater reproducibility it is hoped that the hypothesis will be proven to be correct and that work can move forward looking at deviations from normal erythrocyte behavior. 



\section{References}

1. Leonard KM, Minerick AR. Explorations of ABO-Rh antigen expressions on erythrocyte dielectrophoresis: Changes in cross-over frequency. Electrophoresis 2011;32(18):2512-2522.

2. Alazzam A, Stiharu I, Bhat R, Meguerditchian A-N. Interdigitated comb-like electrodes for continuous separation of malignant cells from blood using dielectrophoresis. Electrophoresis 2011;32(11):1327-1336.

3. Becker FF, Wang XB, Huang Y, Pethig R, Vykoukal J, Gascoyne PRC. The removal of human leukaemia cells from blood using interdigitated microelectrodes. Journal of Physics D: Applied Physics 1994;27(12):2659.

4. Gascoyne P, Pethig R, Satayavivad J, Becker FF, Ruchirawat M. Dielectrophoretic detection of changes in erythrocyte membranes following malarial infection. Biochimica Et Biophysica Acta-Biomembranes $1997 ; 1323(2): 240-252$.

5. Gascoyne P, Satayavivad J, Ruchirawat M. Microfluidic approaches to malaria detection. Acta Tropica 2004;89(3):357-369.

6. Huang Y, Holzel R, Pethig R, Xiao BW. Differences in the AC electrodynamics of viable and non-viable yeast cells determined through combined dielectrophoresis and electrorotation studies. Physics in Medicine and Biology 1992;37(7):1499. 
7. Sano MB, Caldwell JL, Davalos RV. Modeling and development of a low frequency contactless dielectrophoresis (cDEP) platform to sort cancer cells from dilute whole blood samples. Biosensors \& Bioelectronics 2011;30(1):13-20.

8. Hu Q, Joshi RP, Beskok A. Model study of electroporation effects on the dielectrophoretic response of spheroidal cells. Journal of Applied Physics 2009;106(2):8.

9. Sano MB, Henslee EA, Schmelz E, Davalos RV. Contactless dielectrophoretic spectroscopy: Examination of the dielectric properties of cells found in blood. Electrophoresis 2011;32(22):3164-3171.

10. Srivastava SK, Artemiou A, Minerick AR. Direct current insulator-based dielectrophoretic characterization of erythrocytes: ABO-Rh human blood typing. Electrophoresis 2011;32(18):2530-2540.

11. Srivastava SK, Daggolu PR, Burgess SC, Minerick AR. Dielectrophoretic characterization of erythrocytes: Positive ABO blood types. Electrophoresis 2008;29(24):5033-5046.

12. Vykoukal DM, Gascoyne PRC, Vykoukal J. Dielectric characterization of complete mononuclear and polymorphonuclear blood cell subpopulations for label-free discrimination. Integrative Biology 2009;1(7):477-484.

13. Yang J, Huang Y, Wang X-B, Becker FF, Gascoyne PRC. Differential Analysis of Human Leukocytes by Dielectrophoretic Field-Flow-Fractionation. Biophysical Journal 2000;78(5):2680-2689. 
14. Yang J, Huang Y, Wang XJ, Wang XB, Becker FF, Gascoyne PRC. Dielectric properties of human leukocyte subpopulations determined by electrorotation as a cell separation criterion. Biophysical Journal 1999;76(6):3307-3314.

15. Pohl H. Dielectrophoresis. New York, NY: Cambridge University Press; 1978.

16. Cemazar J, Vrtacnik D, Amon S, Kotnik T. Dielectrophoretic Field-Flow Microchamber for Separation of Biological Cells Based on Their Electrical Properties. Ieee Transactions on Nanobioscience 2011;10(1):36-43.

17. Cetin B, Li D. Dielectrophoresis in microfluidics technology. Electrophoresis 2011;32(18):2410-2427.

18. Guido I, Jaeger MS, Duschl C. Dielectrophoretic stretching of cells allows for characterization of their mechanical properties. European Biophysics Journal with Biophysics Letters 2011;40(3):281-288.

19. Imasato H, Yamakawa T. Separation of leukemia cells from blood by employing dielectrophoresis. 2010 World Automation Congress (WAC 2010) 2010.

20. Jones PV, Staton SJR, Hayes MA. Blood cell capture in a sawtooth dielectrophoretic microchannel. Analytical and Bioanalytical Chemistry 2011;401(7):2103-2111.

21. Lei U, Huang CW, Chen J, Yang CY, Lo YJ, Wo A, Chen CF, Fung TW. A travelling wave dielectrophoretic pump for blood delivery. Lab on a Chip 2009;9(10):1349-1356.

22. Luis Sebastiyn J, Mu oz S, Sancho M, Alvarez G. Polarizability of shelled particles of arbitrary shape in lossy media with an application to hematic cells. Physical Review E 2008;78(5). 
23. Minerick AR, Zhou RH, Takhistov P, Chang HC. Manipulation and characterization of red blood cells with alternating current fields in microdevices. Electrophoresis 2003;24(21):3703-3717.

24. Nakashima Y, Hata S, Yasuda T. Blood plasma separation and extraction from a minute amount of blood using dielectrophoretic and capillary forces. Sensors and Actuators B-Chemical 2010;145(1):561-569.

25. Salmanzadeh A, Romero L, Shafiee H, Gallo-Villanueva RC, Stremler MA, Cramer SD, Davalos RV. Isolation of prostate tumor initiating cells (TICs) through their dielectrophoretic signature. Lab on a Chip 2012;12(1):182-189.

26. Dailey JF. Blood. Medical Consulting Group; 1998.

27. Takakuwa A, Ikawa M, Fujita M, Yase K. Micropatterning of Electrodes by Microcontact Printing Method and Application to Thin Film Transistor Devices. Japanese Journal of Applied Physics;46(9A):5960.

28. Flack WW, Soong DS, Bell AT, Hess DW. A mathematical model for spin coating of polymer resists. Journal of Applied Physics 1984;56(4):1199-1206.

29. Polson NA, Hayes MA. Microfluidics - Controlling fluids in small places. Analytical Chemistry 2001;73(11):312A-319A.

30. Beebe DJ, Mensing GA, Walker GM. Physics and applications of microfluidics in biology. Annual Review of Biomedical Engineering 2002;4:261-286.

31. Childs WR, Nuzzo RG. Decal transfer microlithography: A new soft-lithographic patterning method. Journal of the American Chemical Society 2002;124(45):13583-13596. 
32. Manica DR, Ewing AG. Prototyping disposable electrophoresis microchips with electrochemical detection using rapid marker masking and laminar flow etching. Electrophoresis 2002;23(21):3735-3743.

33. McDonald JC, Whitesides GM. Poly(dimethylsiloxane) as a material for fabricating microfluidic devices. Accounts of Chemical Research 2002;35(7):491499.

34. Ng JMK, Gitlin I, Stroock AD, Whitesides GM. Components for integrated poly(dimethylsiloxane) microfluidic systems. Electrophoresis 2002;23(20):34613473.

35. Reyes DR, Iossifidis D, Auroux PA, Manz A. Micro total analysis systems. 1. Introduction, theory, and technology. Analytical Chemistry 2002;74(12):26232636.

36. Fiorini GS, Jeffries GDM, Lim DSW, Kuyper CL, Chiu DT. Fabrication of thermoset polyester microfluidic devices and embossing masters using rapid prototyped polydimethylsiloxane molds. Lab on a Chip 2003;3(3):158-163.

37. Rodriguez I, Spicar-Mihalic P, Kuyper CL, Fiorini GS, Chiu DT. Rapid prototyping of glass microchannels. Analytica Chimica Acta 2003;496(1-2):205215.

38. Verpoorte E, De Rooij NF. Microfluidics meets MEMS. Proceedings of the Ieee 2003;91(6):930-953.

39. Zhang LH, Dang FQ, Baba Y. Microchip electrophoresis-based separation of DNA. Journal of Pharmaceutical and Biomedical Analysis 2003;30(6):1645-1654. 
40. Berdichevsky Y, Khandurina J, Guttman A, Lo YH. UV/ozone modification of poly(dimethylsiloxane) microfluidic channels. Sensors and Actuators B-Chemical 2004;97(2-3):402-408.

41. Chen L, Ren JC. High-throughput DNA analysis by microchip electrophoresis. Combinatorial Chemistry \& High Throughput Screening 2004;7(1):29-43.

42. Fiorini GS, Lorenz RM, Kuo JS, Chiu DT. Rapid prototyping of thermoset polyester microfluidic devices. Analytical Chemistry 2004;76(16):4697-4704.

43. Jia ZJ, Fang Q, Fang ZL. Bonding of glass microfluidic chips at room temperatures. Analytical Chemistry 2004;76(18):5597-5602.

44. Fiorini GS, Chiu DT. Disposable microfluidic devices: fabrication, function, and application. Biotechniques 2005;38(3):429-446.

45. Mao P, Han JY. Fabrication and characterization of $20 \mathrm{~nm}$ planar nanofluidic channels by glass-glass and glass-silicon bonding. Lab on a Chip 2005;5(8):837844.

46. Metzker ML. Emerging technologies in DNA sequencing. Genome Research 2005;15(12):1767-1776.

47. Squires TM, Quake SR. Microfluidics: Fluid physics at the nanoliter scale. Reviews of Modern Physics 2005;77(3):977-1026.

48. Sudarsan AP, Wang J, Ugaz VM. Thermoplastic elastomer gels: An advanced substrate for microfluidic chemical analysis systems. Analytical Chemistry 2005;77(16):5167-5173.

49. Szekely L, Guttman A. New advances in microchip fabrication for electrochromatography. Electrophoresis 2005;26(24):4590-4604. 
50. Wu HK, Huang B, Zare RN. Construction of microfluidic chips using polydimethylsiloxane for adhesive bonding. Lab on a Chip 2005;5(12):1393-1398.

51. An YH, Song SM. Fabrication of a CNT filter for a microdialysis chip. Molecular \& Cellular Toxicology 2006;2(4):279-284.

52. Chen LX, Luo GA, Liu KH, Ma JP, Yao B, Yan YC, Wang YM. Bonding of glass-based microfluidic chips at low- or room-temperature in routine laboratory. Sensors and Actuators B-Chemical 2006;119(1):335-344.

53. Dittrich PS, Tachikawa K, Manz A. Micro total analysis systems. Latest advancements and trends. Analytical Chemistry 2006;78(12):3887-3907.

54. Liu CC, Cui DF, Cai HY, Chen X, Geng ZX. A rigid poly(dimethylsiloxane) sandwich electrophoresis microchip based on thin-casting method. Electrophoresis 2006;27(14):2917-2923.

55. Mair DA, Geiger E, Pisano AP, Frechet JMJ, Svec F. Injection molded microfluidic chips featuring integrated interconnects. Lab on a Chip 2006;6(10):1346-1354.

56. Pan YJ, Yang RJ. A glass microfluidic chip adhesive bonding method at room temperature. Journal of Micromechanics and Microengineering 2006;16(12):2666-2672.

57. Addae-Mensah KA, Kassebaum NJ, Bowers MJ, Reiserer RS, Rosenthal SJ, Moore PE, Wikswo JP. A flexible, quantum dot-labeled cantilever post array for studying cellular microforces. Sensors and Actuators a-Physical 2007;136(1):385397. 
58. Akiyama Y, Morishima K, Kogi A, Kikutani Y, Tokeshi M, Kitamori T. Rapid bonding of Pyrex glass microchips. Electrophoresis 2007;28(6):994-1001.

59. Bubendorfer A, Liu XM, Ellis AV. Microfabrication of PDMS microchannels using SU-8/PMMA moldings and their sealing to polystyrene substrates. Smart Materials \& Structures 2007;16(2):367-371.

60. Chen Q, Li G, Jin QH, Zhao JL, Ren QS, Xu YS. A rapid and low-cost procedure for fabrication of glass microfluidic devices. Journal of Microelectromechanical Systems 2007;16(5):1193-1200.

61. Chueh BH, Huh D, Kyrtsos CR, Houssin T, Futai N, Takayama S. Leakage-free bonding of porous membranes into layered microfluidic array systems. Analytical Chemistry 2007;79(9):3504-3508.

62. Coltro WKT, Piccin E, Carrilho E, de Jesus DP, Fracassi da Silva JA, Torres da Silva HD, do Lago CL. Micro chemical analysis systems. Introduction, fabrication technologies, instrumentation and applications. Quimica Nova 2007;30(8):1986-2000.

63. Genes LI, Tolan NV, Hulvey MK, Martin RS, Spence DM. Addressing a vascular endothelium array with blood components using underlying microfluidic channels. Lab on a Chip 2007;7(10):1256-1259.

64. Gu J, Gupta R, Chou CF, Wei QH, Zenhausern F. A simple polysilsesquioxane sealing of nanofluidic channels below $10 \mathrm{~nm}$ at room temperature. Lab on a Chip 2007;7(9):1198-1201.

65. Haeberle S, Zengerle R. Microfluidic platforms for lab-on-a-chip applications. Lab on a Chip 2007;7(9):1094-1110. 
66. Iles A, Oki A, Pamme N. Bonding of soda-lime glass microchips at low temperature. Microfluidics and Nanofluidics 2007;3(1):119-122.

67. Lee K, Kim C, Shin KS, Lee J, Ju BK, Kim TS, Lee SK, Kang JY. Fabrication of round channels using the surface tension of PDMS and its application to a 3D serpentine mixer. Journal of Micromechanics and Microengineering 2007;17(8):1533-1541.

68. Liu HB, Gong HQ, Ramalingam N, Jiang Y, Dai CC, Hui KM. Micro air bubble formation and its control during polymerase chain reaction (PCR) in polydimethylsiloxane (PDMS) microreactors. Journal of Micromechanics and Microengineering 2007;17(10):2055-2064.

69. Maltezos G, Johnston M, Maltezos DG, Scherer A. Replication of threedimensional valves from printed wax molds. Sensors and Actuators a-Physical 2007;135(2):620-624.

70. Matsui T, Franzke J, Manz A, Janasek D. Temperature gradient focusing in a PDMS/glass hybrid microfluidic chip. Electrophoresis 2007;28(24):4606-4611.

71. Pan T, Fiorin GS, Chiu DT, Woolley AT. In-channel atom-transfer radical polymerization of thermoset polyester microfluidic devices for bioanalytical applications. Electrophoresis 2007;28(16):2904-2911.

72. Park JS, Zhu H, Zhao ZL, Tseng AA, Chen TP. Direct writing of spot and line bonds for microsystem packaging using transmission laser bonding technique. Materials and Manufacturing Processes 2007;22(1):71-80. 
73. Patrito N, McLachlan JM, Faria SN, Chan J, Norton PR. A novel metal-protected plasma treatment for the robust bonding of polydimethylsiloxane. Lab on a Chip 2007;7(12):1813-1818.

74. Piccin E, Coltro WKT, da Silva JAF, Neto SC, Mazo LH, Carrilho E.

Polyurethane from biosource as a new material for fabrication of microfluidic devices by rapid prototyping. Journal of Chromatography A 2007;1173(1-2):151158.

75. Samel B, Chowdhury MK, Stemme G. The fabrication of microfluidic structures by means of full-wafer adhesive bonding using a poly(dimethylsiloxane) catalyst. Journal of Micromechanics and Microengineering 2007;17(8):1710-1714.

76. Samel B, Griss P, Stemme G. A thermally responsive PDMS composite and its microfluidic applications. Journal of Microelectromechanical Systems 2007;16(1):50-57.

77. Torisawa YS, Chueh BH, Huh D, Ramamurthy P, Roth TM, Barald KF, Takayama S. Efficient formation of uniform-sized embryoid bodies using a compartmentalized microchannel device. Lab on a Chip 2007;7(6):770-776.

78. Zhao JY, Shang ZP, Gao LX. Bonding quartz wafers by the atom transfer radical polymerization of the glycidyl methacrylate at mild temperature. Sensors and Actuators a-Physical 2007;135(1):257-261.

79. Abdelgawad M, Watson MWL, Young EWK, Mudrik JM, Ungrin MD, Wheeler AR. Soft lithography: masters on demand. Lab on a Chip 2008;8(8):1379-1385.

80. Allen PB, Chiu DT. Calcium-assisted glass-to-glass bonding for fabrication of glass microfluidic devices. Analytical Chemistry 2008;80(18):7153-7157. 
81. Becker H, Gartner C. Polymer microfabrication technologies for microfluidic systems. Analytical and Bioanalytical Chemistry 2008;390(1):89-111.

82. Castano-Alvarez M, Ayuso DFP, Granda MG, Fernandez-Abedul MT, Garcia JR, Costa-Garcia A. Critical points in the fabrication of microfluidic devices on glass substrates. Sensors and Actuators B-Chemical 2008;130(1):436-448.

83. Coltro WKT, Lunte SM, Carrilho E. Comparison of the analytical performance of electrophoresis microchannels fabricated in PDMS, glass, and polyester-toner. Electrophoresis 2008;29(24):4928-4937.

84. Gao YX, Chen LW. Versatile control of multiphase laminar flow for in-channel microfabrication. Lab on a Chip 2008;8(10):1695-1699.

85. Hanada Y, Sugioka K, Kawano H, Ishikawa IS, Miyawaki A, Midorikawa K. Nano-aquarium for dynamic observation of living cells fabricated by femtosecond laser direct writing of photostructurable glass. Biomedical Microdevices 2008;10(3):403-410.

86. Kamotani Y, Bersano-Begey T, Kato N, Tung YC, Huh D, Song JW, Takayama S. Individually programmable cell stretching microwell arrays actuated by a Braille display. Biomaterials 2008;29(17):2646-2655.

87. Li PY, Shih J, Lo R, Saati S, Agrawal R, Humayun MS, Tai YC, Meng E. An electrochemical intraocular drug delivery device. 2008. p 41-48.

88. Lo R, Li PY, Saati S, Agrawal R, Humayun MS, Meng E. A refillable microfabricated drug delivery device for treatment of ocular diseases. Lab on a Chip 2008;8(7):1027-1030. 
89. Luo YQ, Yu F, Zare RN. Microfluidic device for immunoassays based on surface plasmon resonance imaging. Lab on a Chip 2008;8(5):694-700.

90. Luo YQ, Zare RN. Perforated membrane method for fabricating threedimensional polydimethylsiloxane microfluidic devices. Lab on a Chip 2008;8(10):1688-1694.

91. Ou JJ, Glawdel T, Samy R, Wang SW, Liu Z, Ren CL, Pawliszyn J. Integration of dialysis membranes into a poly(dimethylsiloxane) microfluidic chip for isoelectric focusing of proteins using whole-channel imaging detection. Analytical Chemistry 2008;80(19):7401-7407.

92. Pandolfi A, Ortiz M. Numerical analysis of elastomeric fluidic microvalves. 2008. p 43-48.

93. Suk JW, Jang JY, Cho JH. Reagent-loaded plastic microfluidic chips for detecting homocysteine. Journal of Micromechanics and Microengineering 2008;18(5).

94. Truxal SC, Tung YC, Kurabayashi K. A flexible nanograting integrated onto silicon micromachines by soft lithographic replica molding and assembly. Journal of Microelectromechanical Systems 2008;17(2):393-401.

95. Wu DP, Qin JH, Lin BC. Electrophoretic separations on microfluidic chips. Journal of Chromatography A 2008;1184(1-2):542-559.

96. Wu ZM, Li DQ. Mixing and flow regulating by induced-charge electrokinetic flow in a microchannel with a pair of conducting triangle hurdles. Microfluidics and Nanofluidics 2008;5(1):65-76.

97. Yun KS, Yoon E. Fabrication of complex multilevel microchannels in PDMS by using three-dimensional photoresist masters. Lab on a Chip 2008;8(2):245-250. 
98. Zhang MJ, Zhao HY, Gao LX. Glass wafers bonding via Diels-Alder reaction at mild temperature. Sensors and Actuators a-Physical 2008;141(1):213-216.

99. Gong XQ, Wen WJ. Polydimethylsiloxane-based conducting composites and their applications in microfluidic chip fabrication. 2009.

100. Im SG, Bong KW, Lee CH, Doyle PS, Gleason KK. A conformal nano-adhesive via initiated chemical vapor deposition for microfluidic devices. Lab on a Chip 2009;9(3):411-416.

101. Kuo JS, Ng LY, Yen GS, Lorenz RM, Schiro PG, Edgar JS, Zhao YX, Lim DSW, Allen PB, Jeffries GDM and others. A new USP Class VI-compliant substrate for manufacturing disposable microfluidic devices. Lab on a Chip 2009;9(7):870-876.

102. Lee NY, Chung BH. Novel Poly(dimethylsiloxane) Bonding Strategy via Room Temperature "Chemical Gluing". Langmuir 2009;25(6):3861-3866.

103. Liu HB, Gong HQ. Templateless prototyping of polydimethylsiloxane microfluidic structures using a pulsed CO2 laser. Journal of Micromechanics and Microengineering 2009;19(3).

104. Subramani BG, Selvaganapathy PR. Surface micromachined PDMS microfluidic devices fabricated using a sacrificial photoresist. 2009.

105. Tolan NV, Genes LI, Subasinghe W, Raththagala M, Spence DM. Personalized Metabolic Assessment of Erythrocytes Using Microfluidic Delivery to an Array of Luminescent Wells. Analytical Chemistry 2009;81(8):3102-3108.

106. van Noort D, Ong SM, Zhang C, Zhang SF, Arooz T, Yu H. Stem Cells in Microfluidics. Biotechnology Progress 2009;25(1):52-60. 
107. Zheng YZ, Dai W, Wu HK. A screw-actuated pneumatic valve for portable, disposable microfluidics. Lab on a Chip 2009;9(3):469-472.

108. El-Ali J, Sorger PK, Jensen KF. Cells on chips. Nature 2006;442(7101):403-411.

109. Wang HY, Lu C. Microfluidic electroporation for delivery of small molecules and genes into cells using a common DC power supply. Biotechnology and Bioengineering 2008;100(3):579-586.

110. Becker FF, Wang XB, Huang Y, Pethig R, Vykoukal J, Gascoyne PRC. THE REMOVAL OF HUMAN LEUKEMIA-CELLS FROM BLOOD USING INTERDIGITATED MICROELECTRODES. Journal of Physics D-Applied Physics 1994;27(12):2659-2662.

111. Martinez-Duarte R. Microfabrication technologies in dielectrophoresis applications - a review. Electrophoresis 2012:n/a-n/a.

112. Chang H-C, Yeo L. Electrokinetically-Driven Microfluidics and Nanofluidics. New York, NY: Cambridge University Press; 2009.

113. Srivastava SK, Gencoglu A, Minerick AR. DC insulator dielectrophoretic applications in microdevice technology: a review. Analytical and Bioanalytical Chemistry 2011;399(1):301-321.

114. Jones TB. Electromechanics of Particles. Cambridge, UK: Cambridge University Press; 1995.

115. Kirby B. Micro- and Nanoscale Fluid Mechanics: Transport in Microfluidic Devices. New York, NY: Cambridge University Press; 2010.

116. Menachery A, Pethig R. Controlling cell destruction using dielectrophoretic forces. Iee Proceedings-Nanobiotechnology 2005;152(4):145-149. 
117. Morgan H, Green NG. AC Electrokinetics: Colloids and Nanoparticles. Pethig R, editor. Baldock, Hertfordshire, England: Research Studies Press Ltd; 2003.

118. Pethig R, Bressler V, Carswell-Crumpton C, Chen Y, Foster-Haje L, GarciaOjeda ME, Lee RS, Lock GM, Talary MS, Tate KM. Dielectrophoretic studies of the activation of human $\mathrm{T}$ lymphocytes using a newly developed cell profiling system. Electrophoresis 2002;23(13):2057-2063.

119. Landsteiner K. Zur Kenntnis def antifementativen, lytischen und agglutinierenden Wirkungen des Bluteserums und der Lumphe. ZB1. Bakt. 1900;27:357-362.

120. Daniels G, Bromilow I. Essential Guide to Blood Groups. Malden, MA: Blackwell Publishing; 2008.

121. Reid ME, Lomas-Francis C. The Blood Group Antigen Facts Book, 2nd Edition. London, UK: Elsevier Ltd.; 2007.

122. Minerick AR. The rapidly growing field of micro and nanotechnology to measure living cells. Aiche Journal 2008;54(9):2230-2237.

123. Gagnon Z, Gordon J, Sengupta S, Chang HC. Bovine red blood cell starvation age discrimination through a glutaraldehyde-amplified dielectrophoretic approach with buffer selection and membrane cross-linking. Electrophoresis 2008;29(11):2272-2279.

124. Gordon JE, Gagnon Z, Chang HC. Dielectrophoretic discrimination of bovine red blood cell starvation age by buffer selection and membrane cross-linking. Biomicrofluidics 2007;1(4):5.

125. Global blood safety and availability [Internet]. 2009 [updated cited Available from: http://www.who.int/mediacentre/factsheets/fs279/en/index.html 
126. Langelier SM, Livak-Dahl E, Manzo AJ, Johnson BN, Walter NG, Burns MA. Flexible casting of modular self-aligning microfluidic assembly blocks. Lab on a Chip 2011;11(9):1679-1687.

127. Table of Blood Group Systems [Internet]. 2006 [updated cited Available from: http://ibgrl.blood.co.uk/isbt\%20pages/isbt\%20terminology\%20pages/table\%20of \%20blood\%20group\%20systems.htm

128. Pethig R. Dielectrophoresis: Using Inhomogeneous AC Electric Fields to Separate and Manipulate Cells. Crit. Rev. Biotech 1996;16(4):341-348.

129. Hughes M. Strategies for dielectrophoretic separation in laboratory-on-a-chip systems. Electrophoresis 2002;23:2569-2582.

130. Tortora GJ, Derrickson B. Principles of Anatomy. Danvers, MA: John Wiley \& Sons Inc; 2006.

131. Pallister C. Blood: Physiologyy and Pathophysiology. London, UK: ButterworthHeinemann Ltd; 1994.

132. Parham P. The Immune System. New York, NY: Garland Science, Taylor \&Francis Group; 1950.

133. Visser KR. Electric conductivity of stationary and flowing human blood at low frequencies. 1989 9-12 Nov 1989. p 1540-1542 vol.5.

134. Clancy J. Basic Concepts in Immunology: A Student's Survival Guide. New York, NY: McGraw-Hill Companies; 1998.

135. Mohandas N, Gallagher PG. Red cell membrane: past, prasent and future. Blood 2008;112(10):3939-3948 . 
136. Gorter E, Grendel F. ON BIMOLECULAR LAYERS OF LIPOIDS ON THE CHROMOCYTES OF THE BLOOD. The Journal of experimental medicine $1925 ; 41(4): 439-43$.

137. Marchesi VT, Steers E, Jr. Selective solubilization of a protein component of the red cell membrane. Science 1968;159(3811):203-4.

138. Steck TL, Fairbanks G, Wallach DF. Disposition of the major proteins in the isolated erythrocyte membrane. Proteolytic dissection. Biochemistry $1971 ; 10(13): 2617-24$.

139. Singer SJ, Nicolson GL. The fluid mosaic model of the structure of cell membranes. Science 1972;175(4023):720-31.

140. Gimsa J, Muller T, Schnelle T, Fuhr G. Dielectric spectroscopy of single human erythrocytes at physiological ionic strength: Dispersion of the cytoplasm. Biophysical Journal 1996;71(1):495-506.

141. Vissers MCM, Carr AC, Chapman ALP. Comparison of human red cell lysis by hypochlorous and hypobromous acids: insights into the mechanism of lysis. Biochemical Journal 1998;330:131-138.

142. Dean L. Blood Groups and Red Cell Antigens. Bethesda, MD: National Center for Biotechnology Information; 2005.

143. Liu Z, Peng JB, Mo R, Hui CC, Huang CH. Rh type B glycoprotein is a new member of the Rh superfamily and a putative ammonia transporter in mammals. Journal of Biological Chemistry 2001;276(2):1424-1433.

144. Tang Y-W, Stratton CW, Campbell S, Landry M. Rapid Antigen Tests Advanced Techniques in Diagnostic Microbiology. Springer US; 2006. p 23-41. 
145. Winograd E, Sherman IW. CHARACTERIZATION OF A MODIFIED REDCELL MEMBRANE-PROTEIN EXPRESSED ON ERYTHROCYTES INFECTED WITH THE HUMAN MALARIA PARASITE PLASMODIUMFALCIPARUM - POSSIBLE ROLE AS A CYTOADHERENT MEDIATING PROTEIN. Journal of Cell Biology 1989;108(1):23-30.

146. Aikawa M. VARIATIONS IN STRUCTURE AND FUNCTION DURING LIFECYCLE OF MALARIAL PARASITES. Bulletin of the World Health Organization 1977;55(2-3):139-156.

147. Miller LH. The ultrastructure of red cells infected by Plasmodium falciparum in man. Transactions of the Royal Society of Tropical Medicine and Hygiene 1972;66(3):459-62.

148. Vernot-Hernandez JP, Heidrich HG. THE RELATIONSHIP TO KNOBS OF THE 92,000 D-PROTEIN SPECIFIC FOR KNOBBY STRAINS OF PLASMODIUM-FALCIPARUM. Zeitschrift Fur Parasitenkunde-Parasitology Research 1985;71(1):41-51.

149. Menachery A, Pethig R. Controlling cell destruction using dielectrophoretic forces. 2005 .

150. Wang X, Wang X-B, Gascoyne PRC. General expressions for dielectrophoretic force and electrorotational torque derived using the Maxwell stress tensor method. Journal of Electrostatics 1997;39:277-295.

151. Eda K, Eda S, Sherman IW. Identification of peptides targeting the surface of Plasmodium falciparum-infected erythrocytes using a phage display peptide library. American Journal of Tropical Medicine and Hygiene 2004;71(2):190-195. 
152. Kay MM. Band 3 and its alterations in health and disease. Cellular and Molecular Biology 2004;50(2):117-138.

153. Pantaleo A, De Franceschi L, Ferru E, Vono R, Turrini F. Current knowledge about the functional roles of phosphorylative changes of membrane proteins in normal and diseased red cells. Journal of Proteomics 2010;73(3):445-455.

154. Pantaleo A, Ferru E, Carta F, Mannu F, Giribaldi G, Vono R, Lepedda AJ, Pippia P, Turrini F. Analysis of changes in tyrosine and serine phosphorylation of red cell membrane proteins induced by P. falciparum growth. Proteomics 2010;10(19):3469-3479.

155. Smith JD, Craig AG. The surface of the Plasmodium falciparum-infected erythrocyte. Current Issues in Molecular Biology 2005;7:81-93.

156. Winograd E, Prudhomme JG, Sherman IW. Band 3 clustering promotes the exposure of neoantigens in Plasmodium falciparum-infected erythrocytes. Molecular and Biochemical Parasitology 2005;142(1):98-105.

157. Cetin B, Li DQ. Continuous particle separation based on electrical properties using alternating current dielectrophoresis. Electrophoresis 2009;30(18):31243133.

158. Cetin B, Li DQ. Lab-on-a-chip device for continuous particle and cell separation based on electrical properties via alternating current dielectrophoresis. Electrophoresis 2010;31(18):3035-3043.

159. Chen DF, Du H, Li WH. Bioparticle separation and manipulation using dielectrophoresis. Sensors and Actuators A: Physical 2007;133(2):329-334. 
160. Chen DF, Du HJ. A dielectrophoretic barrier-based microsystem for separation of microparticles. Microfluidics and Nanofluidics 2007;3(5):603-610.

161. Fatoyinbo HO, Hoeftges KF, Hughes MP. Rapid-on-chip determination of dielectric properties of biological cells using imaging techniques in a dielectrophoresis dot microsystem. Electrophoresis 2008;29(1):3-10.

162. Gagnon Z, Mazur J, Chang HC. Glutaraldehyde enhanced dielectrophoretic yeast cell separation. Biomicrofluidics 2009;3(4).

163. Gascoyne PRC. Dielectrophoretic-Field Flow Fractionation Analysis of Dielectric, Density, and Deformability Characteristics of Cells and Particles. Analytical Chemistry 2009;81(21):8878-8885.

164. Gascoyne PRC, Vkkoukal J. Particle Separation by Dielectrophoresis. Electrophoresis 2002;23:1973-1983.

165. Gimsa J. Particle characterization by AC-electrokinetic phenomena: 1. A short introduction to dielectrophoresis (DP) and electrorotation (ER). Colloids and Surfaces A: Physicochemical and Engineering Aspects 1999;149:451-459.

166. Green NG, Morgan H. Separation of submicrometre particles using a combination of dielectrophoretic and electrohydrodynamic forces. Journal of Physics D: Applied Physics 1998;31(7):L25.

167. Green NG, Morgan H. Dielectrophoresis of Submicrometer Latex Spheres. 1. Experimental Results. The Journal of Physical Chemistry B 1999;103(1):41-50.

168. Hawkins BG, Smith AE, Syed YA, Kirby BJ. Continuous-flow particle separation by 3D insulative dielectrophoresis using coherently shaped, dc-biased, ac electric fields. Analytical Chemistry 2007;79(19):7291-7300. 
169. Hubner Y, Hoettges KF, Kass GEN, Ogin SL, Hughes MP. Parallel measurements of drug actions on erythrocytes by dielectrophoresis, using a three-dimensional electrode design. Nanobiotechnology, IEE Proceedings - 2005;152(4):150-154.

170. Hughes MP, Morgan H, Rixon FJ. Dielectrophoretic manipulation and characterization of herpes simplex virus-1 capsids. European Biophysics Journal with Biophysics Letters 2001;30(4):268-272.

171. Hughes MP, Morgan H, Rixon FJ. Measuring the dielectric properties of herpes simplex virus type 1 virions with dielectrophoresis. Biochimica Et Biophysica Acta-General Subjects 2002;1571(1):1-8.

172. Nascimento EM, Nogueira N, Silva T, Braschler T, Demierre N, Renaud P, Oliva AG. Dielectrophoretic sorting on a microfabricated flow cytometer: Label free separation of Babesia bovis infected erythrocytes. Bioelectrochemistry 2008;73(2):123-128.

173. Pethig R. Dielectrophoresis: Status of the theory, technology, and applications (vol 4, 022811, 2010). Biomicrofluidics 2010;4(3).

174. Newman J, Thomas-Alyea KE. Electrochemical Systems. Hoboken, NJ: John Wiley \& Sons, Inc; 2004.

175. Wang X, Huang Y, Gascoyne PRC, Becker FF. Dielectrophoretic Manipulation of Particles. IEEE Transactions on Industry Applications 1997;33:660-669.

176. Ivorra A, Villemejane J, Mir LM. Electrical modeling of the influence of medium conductivity on electroporation. Physical Chemistry Chemical Physics;12(34):10055-10064. 
177. Kay MMB, Flowers N, Goodman J, Bosman G. ALTERATION IN

MEMBRANE-PROTEIN BAND-3 ASSOCIATED WITH ACCELERATED

ERYTHROCYTE AGING. Proceedings of the National Academy of Sciences of the United States of America 1989;86(15):5834-5838.

178. Selim NS, Desouky OS, Elbakrawy EM, Rezk RA. Electrical behavior of stored erythrocytes after exposure to gamma radiation and the role of alpha-lipoic acid as radioprotector. Appl Radiat Isot;68(6):1018-24.

179. Wang M-H, Jang L-S. A systematic investigation into the electrical properties of single HeLa cells via impedance measurements and COMSOL simulations.

Biosensors and Bioelectronics 2009;24(9):2830-2835.

180. Martinsen O, Grimnes S, Schwan HP. Interface of Phenomena and Dielectric Properties of Biological Tissue. Encyclopedia of Surface and Colloid Science 2002:2643-2652.

181. Maxwell JC. Treatise on Electricity and Magnetism. Oxford University Press; 1873.

182. Fricke H. The complex conductivity of a suspension of stratified particles of spherical cylindrical form. J. Phys. Chem 1955;59.

183. Carrique F, Arroyo FJ, Shilov VN, Cuquejo J, Jimenez ML, Delgado AV. Effect of stagnant-layer conductivity on the electric permittivity of concentrated colloidal suspensions. Journal of Chemical Physics 2007;126(10).

184. Carrique F, Ruiz-Reina E, Arroyo FJ, Jimenez ML, Delgado AV. Dielectric Response of a Concentrated Colloidal Suspension in a Salt-Free Medium. Langmuir 2008;24(20):11544-11555. 
185. Carrique F, Ruiz-Reina E, Arroyo FJ, Jimenez ML, Delgado AV. Dynamic electrophoretic mobility of spherical colloidal particles in salt-free concentrated suspensions. Langmuir 2008;24(6):2395-2406.

186. Froude VE, Zhu YX. Dielectrophoresis of Functionalized Lipid Unilamellar Vesicles (Liposomes) with Contrasting Surface Constructs. Journal of Physical Chemistry B 2009;113(6):1552-1558.

187. Huang Y, Pethig R. ELECTRODE DESIGN FOR NEGATIVE DIELECTROPHORESIS. Measurement Science \& Technology 1991;2(12):11421146.

188. Carrique F, Cuquejo J, Arroyo FJ, Jimenez ML, Delgado AV. Influence of cellmodel boundary conditions on the conductivity and electrophoretic mobility of concentrated suspensions. Advances in Colloid and Interface Science 2005;118(1$3): 43-50$.

189. Carrique F, Ruiz-Reina E, Arroyo FJ, Delgado AV. Cell model of the direct current electrokinetics in salt-free concentrated suspensions: The role of boundary conditions. Journal of Physical Chemistry B 2006;110(37):18313-18323.

190. Chan KL, Morgan H, Morgan E, Cameron IT, Thomas MR. Measurements of the dielectric properties of peripheral blood mononuclear cells and trophoblast cells using AC electrokinetic techniques. Biochimica Et Biophysica Acta-Molecular Basis of Disease 2000;1500(3):313-322.

191. Gagnon ZR. Cellular dielectrophoresis: Applications to the characterization, manipulation, separation and patterning of cells. Electrophoresis 2011;32(18):2466-2487. 
192. Hywel M, et al. Single cell dielectric spectroscopy. Journal of Physics D: Applied Physics 2007;40(1):61.

193. Ouellette AL, Li JJ, Cooper DE, Ricco AJ, Kovacs GTA. Evolving Point-of-Care Diagnostics Using Up-Converting Phosphor Bioanalytical Systems. Analytical Chemistry 2009;81(9):3216-3221.

194. Kurlander RJ, Rosse WF. Lymphocyte-mediated lysis of antibody coated human red cells in the presence of human serum. Blood 1979;53(6):1197-202.

195. Lloyd MM, van Reykt DM, Davies MJ, Hawkins CL. Hypothiocyanous acid is a more potent inducer of apoptosis and protein thiol depletion in murine macrophage cells than hypochlorous acid or hypobromous acid. Biochemical Journal 2008;414:271-280.

196. Majno G, Joris I. APOPTOSIS, ONCOSIS, AND NECROSIS - AN OVERVIEW OF CELL-DEATH. American Journal of Pathology 1995;146(1):3-15.

197. Huang W-H, Ai F, Wang Z-L, Cheng J-K. Recent advances in single-cell analysis using capillary electrophoresis and microfluidic devices. Journal of Chromatography B 2008;866(1-2):104-122.

198. Irimia D, Tompkins RG, Toner M. Single-Cell Chemical Lysis in Picoliter-Scale Closed Volumes Using a Microfabricated Device. Analytical Chemistry 2004;76(20):6137-6143.

199. Sasuga Y, Iwasawa T, Terada K, Oe Y, Sorimachi H, Ohara O, Harada Y. SingleCell Chemical Lysis Method for Analyses of Intracellular Molecules Using an Array of Picoliter-Scale Microwells. Analytical Chemistry 2008;80(23):91419149. 
200. Wu H, Wheeler A, Zare RN. Chemical cytometry on a picoliter-scale integrated microfluidic chip. Proceedings of the National Academy of Sciences of the United States of America 2004;101(35):12809-12813.

201. Price AK, Culbertson CT. Chemical Analysis of Single Mammalian Cells with Microfluidics. Analytical Chemistry 2007;79(7):2614-2621.

202. Di Carlo D, Jeong KH, Lee LP. Reagentless mechanical cell lysis by nanoscale barbs in microchannels for sample preparation. Lab on a Chip 2003;3(4):287-291.

203. Han F, Wang Y, Sims CE, Bachman M, Chang R, Li GP, Allbritton NL. Fast Electrical Lysis of Cells for Capillary Electrophoresis. Analytical Chemistry 2003;75(15):3688-3696.

204. Huang Y, Ewalt KL, Tirado M, Haigis TR, Forster A, Ackley D, Heller MJ, O'Connell JP, Krihak M. Electric manipulation of bioparticles and macromolecules on microfabricated electrodes. Analytical Chemistry 2001;73(7):1549-1559.

205. Huang Y, Rubinsky B. Microfabricated electroporation chip for single cell membrane permeabilization. Sensors and Actuators a-Physical 2001;89(3):242249.

206. Huang Y, Rubinsky B. Flow-through micro-electroporation chip for high efficiency single-cell genetic manipulation. 2003. Elsevier Science Sa. p 205-212.

207. Lee S-W, Tai Y-C. A micro cell lysis device. Sensors and Actuators A: Physical 1999;73(1-2):74-79.

208. Lin YC, Huang MY. Electroporation microchips for in vitro gene transfection. Journal of Micromechanics and Microengineering 2001;11(5):542-547. 
209. Lin YC, Jen CM, Huang MY, Wu CY, Lin XZ. Electroporation microchips for continuous gene transfection. Sensors and Actuators B-Chemical 2001;79(23):137-143.

210. Lu H, Schmidt MA, Jensen KF. A microfluidic electroporation device for cell lysis. Lab on a Chip 2005;5(1):23-29.

211. Nandakumar MP, Marten MR. Comparison of lysis methods and preparation protocols for one- and two-dimensional electrophoresis of $<\mathrm{B}><\mathrm{I}>$ Aspergillus oryzae $</ \mathrm{I}></ \mathrm{B}>$ intracellular proteins. Electrophoresis 2002;23(14):2216-2222.

212. Nashimoto Y, Takahashi Y, Yamakawa T, Torisawa Y-s, Yasukawa T, Ito-Sasaki T, Yokoo M, Abe H, Shiku H, Kambara H and others. Measurement of Gene Expression from Single Adherent Cells and Spheroids Collected Using Fast Electrical Lysis. Analytical Chemistry 2007;79(17):6823-6830.

213. Sims CE, Meredith GD, Krasieva TB, Berns MW, Tromberg BJ, Allbritton NL. Laser Micropipet Combination for Single-Cell Analysis. Analytical Chemistry 1998;70(21):4570-4577.

214. Wang H-Y, Bhunia AK, Lu C. A microfluidic flow-through device for high throughput electrical lysis of bacterial cells based on continuous dc voltage. Biosensors and Bioelectronics 2006;22(5):582-588.

215. Wang H-Y, Lu C. Electroporation of Mammalian Cells in a Microfluidic Channel with Geometric Variation. Analytical Chemistry 2006;78(14):5158-5164.

216. Weaver JC. Electroporation of cells and tissues. 2000. Ieee-Inst Electrical Electronics Engineers Inc. p 24-33. 
217. Le Gac S, Zwaan E, van den Berg A, Ohl CD. Sonoporation of suspension cells with a single cavitation bubble in a microfluidic confinement. Lab on a Chip 2007;7(12):1666-1672.

218. Lee JG, Cheong KH, Huh N, Kim S, Choi JW, Ko C. Microchip-based one step DNA extraction and real-time PCR in one chamber for rapid pathogen identification. Lab on a Chip 2006;6(7):886-895.

219. Nevill JT, Cooper R, Dueck M, Breslauer DN, Lee LP. Integrated microfluidic cell culture and lysis on a chip. Lab on a Chip 2007;7(12):1689-1695.

220. Ramadan Q, Samper V, Poenar D, Liang Z, Yu C, Lim TM. Simultaneous cell lysis and bead trapping in a continuous flow microfluidic device. Sensors and Actuators B-Chemical 2006;113(2):944-955.

221. Sun Y, Yin XF. Novel multi-depth microfluidic chip for single cell analysis. Journal of Chromatography A 2006;1117(2):228-233.

222. West J, Becker M, Tombrink S, Manz A. Micro total analysis systems: Latest achievements. Analytical Chemistry 2008;80(12):4403-4419.

223. McDonald JC, Duffy DC, Anderson JR, Chiu DT, Olivier HW, Schueller JA, Whitesides GM. Fabrication of microfluidic systems in poly(dimethylsiloxane). Electrophoresis 2000;21(1):27-40.

224. Duffy DC, McDonald JC, Schueller OJA, Whitesides GM. Rapid Prototyping of Microfluidic Systems in Poly(dimethylsiloxane). Analytical Chemistry 1998;70(23):4974-4984. 
225. Gencoglu A, Minerick A. Chemical and morphological changes on platinum microelectrode surfaces in AC and DC fields with biological buffer solutions. Lab on a Chip 2009;9(13):1866-1873.

226. Braschler T, Demierre N, Nascimento E, Silva T, Oliva AG, Renaud P. Continuous separation of cells by balanced dielectrophoretic forces at multiple frequencies. Lab on a Chip 2008;8(2):280-286.

227. Oblak J, Krizaj D, Amon S, Macek-Lebar A, Miklavcic D. Feasibility study for cell electroporation detection and separation by means of dielectrophoresis. Bioelectrochemistry 2007;71(2):164-171.

228. Vykoukal J, Vykoukal DM, Sharma S, Becker FF, Gascoyne PRC. Dielectrically addressable microspheres engineered using self-assembled monolayers. Langmuir 2003;19(6):2425-2433 .

229. Iwasaki K-i, Nakajima M, Nakao S-i. Galacto-oligosaccharide production from lactose by an enzymic batch reaction using [beta]-galactosidase. Process Biochemistry 1996;31(1):69-76.

230. Park AR, Oh DK. Galacto-oligosaccharide production using microbial betagalactosidase: current state and perspectives. Applied Microbiology and Biotechnology 2010;85(5):1279-1286.

231. Kawabata T, Nakai K, Hagiwara C, Kurokawa N, Murata K, Yaginuma K, Satoh H. Comparison of long-chain polyunsaturated Fatty acids in plasma and erythrocyte phospholipids for biological monitoring. Nippon Eiseigaku Zasshi 2011;66(1):108-114. 
232. Veale MF, Healey G, Sparrow RL. Effect of additive solutions on red blood cell (RBC) membrane properties of stored RBCs prepared from whole blood held for 24 hours at room temperature. Transfusion 2011;51:25S-33S.

233. Gascoyne P, Satayavivad J, Ruchirawat M. Acta Trop. 2004;89:357.

234. Gao J, Sin MLY, Liu T, Gau V, Liao JC, Wong PK. Hybrid electrokinetic manipulation in high-conductivity media. Lab on a Chip 2011;11:1770-1775.

235. Feng J-J, Zhao G, Xu J-J, Chen H-Y. Direct electrochemistry and electrocatalysis of heme proteins immobilized on gold nanoparticles stabilized by chitosan. Analytical Biochemistry 2005;342(2):280-286.

236. Sun J, Loehr TM, Wilks A, Ortiz de Montellano PR. Identification of Histidine 25 as the Heme Ligand in Human Liver Heme Oxygenase. Biochemistry 1994;33(46):13734-13740.

237. Kabanova S, Kleinbongard P, Volkmer J, Andree B, Kelm M, Jax TW. Gene expression analysis of human red blood cells. International Journal of Medical Sciences 2009;6(4):156-159.

238. Kay MMB, Bosman G, Johnson RC, Poulin J, Lawrence C, Goodman J. MOLECULAR-BASIS OF HUMAN BAND-3 MUTATION ASSOCIATED WITH INCREASED ANION TRANSPORT. Experimental and Clinical Immunogenetics 1994;11(4):209-221.

239. Seppi C, Castellana MA, Minetti G, Piccinini G, Balduini C, Brovelli A. EVIDENCE FOR MEMBRANE-PROTEIN OXIDATION DURING INVIVO AGING OF HUMAN ERYTHROCYTES. Mechanisms of Ageing and Development 1991;57(3):247-258. 
240. White CM, Holland LA, Famouri P. Application of capillary electrophoresis to predict crossover frequency of polystyrene particles in dielectrophoresis.

Electrophoresis 2010;31(15):2664-71.

241. Auerswald J, Knapp HF. Quantitative assessment of dielectrophoresis as a micro fluidic retention and separation technique for beads and human blood erythrocytes. Microelectronic Engineering 2003;67-68:879-886.

242. Green NG, Morgan H. Dielectrophoresis of Submicrometer Latex Spheres. 1. Experimental Results. The Journal of Physical Chemistry B 1998;103(1):41-50.

243. Humble RJ. Blood Types in Cattle. Can J Comp Med Vet Sci. 1954;18(11):379389.

\section{Appendices}

\subsection{Experimental Matrices}

\subsubsection{Chapter 6}

Example Matrix for one blood type $\mathrm{A}(+)$. This is repeated for all 8 blood types: $\mathrm{A}+, \mathrm{A}-$, $\mathrm{B}+, \mathrm{B}-, \mathrm{AB}^{+}, \mathrm{AB}-, \mathrm{O}+$ and $\mathrm{O}-$.

\begin{tabular}{|c|c|c|c|}
\hline $\begin{array}{c}\text { Blood } \\
\text { Type }\end{array}$ & Donor \# & Conductivity & Native/Modified \\
\hline \multirow{4}{*}{ A+ } & \multirow{2}{*}{1} & 0.1 & Native \\
\cline { 3 - 4 } & & 0.01 & Modified \\
\cline { 3 - 4 } & 2 & 0.1 & Native \\
\cline { 3 - 4 } & 2 & Natived \\
\hline
\end{tabular}




\begin{tabular}{|c|c|c|}
\hline & & Modified \\
\hline & \multirow{2}{*}{0.01} & Native \\
\hline & & Modified \\
\hline \multirow{4}{*}{3} & \multirow{2}{*}{0.1} & Native \\
\hline & & Modified \\
\hline & \multirow{2}{*}{0.01} & Native \\
\hline & & Modified \\
\hline \multirow{4}{*}{4} & \multirow{2}{*}{0.1} & Native \\
\hline & & Modified \\
\hline & \multirow{2}{*}{0.01} & Native \\
\hline & & Modified \\
\hline \multirow{4}{*}{ Repeat } & \multirow{2}{*}{0.1} & Native \\
\hline & & Modified \\
\hline & \multirow{2}{*}{0.01} & Native \\
\hline & & Modified \\
\hline
\end{tabular}




\subsection{Fabrication Instructions}

Written by Kaela M. Leonard (portions rewritten from Microfabrication Facility Instructions by Bill Knudsen)

Cleaning Glass Slides:

- Fill clean glass dish with acetone

- Sonicate glass slides in acetone for 10 minutes

- Dry glass slides with kimwipe

Clean Room Procedure- Glass Slides for Electrodes:

- Turn hot plate on to $120^{\circ} \mathrm{C}$

- Setup EVG machine

$\circ$ Check EVG machine for green light

- Turn on EVG machine at key switch and computer CPU

- Open EVG software

- Install appropriate lithography mask (follow along with computer instructions)

- Important parameters:

- Mask thickness: do not change

- Mask size: 5 inch

- Substrate size: 4 inch

- Substrate thickness: $1 \mathrm{~mm}$

- Process time: $10 \mathrm{sec}$ (can be optimized based on specific mask)

- Spin coat glass slide with Futurexx PR-1000 photoresist

$\circ$ Spin at $800 \mathrm{rpm}$ for $15 \mathrm{sec}$ with accl $=100$

- Spin at $800 \mathrm{rpm}$ for $30 \mathrm{sec}$ with accl $=300$

- Pre-bake glass slide at $120^{\circ} \mathrm{C}$ for 30 seconds

- Repeat spinning and pre-baking with two more glass slides before exposure

- Expose to UV light for appropriate amount of time

- Post-bake glass slide at $120^{\circ} \mathrm{C}$ for 30 seconds

- Soak in Futurexx developer for 15 seconds

- Wash with E-pure water

- Dry with Nitrogen

- Repeat post-exposure steps with two more glass slides

RIE/ICP Etch System:

- Sign log book

- Select cancel from touchscreen (remember to always be wearing gloves when touching screen)

- Select "vent chamber"

- Wait until chamber has vented and the lid opens

- Load sample with o-rings surrounding sample

- Select "chamber close" from touchscreen menu 
- $\quad$ Select PR-1 from the files menu

- Turn on oxygen at tank

- Input suitable process parameters from "manual process control" menu

○ RIE: 500

○ ICP: 50

○ $\mathrm{O}_{2}$ flow: 100

○ Time: $15 \mathrm{sec}$

- Pressure: 150

- Once system has pumped down completely run recipe by going to "automatic process control"

- After process has ended put system in standby mode for 5 minutes

- Select "vent chamber"

- Remove sample

- Select "chamber close" and allow system to pump down completely

Six Inch Sputter System:

- Pump up to atmosphere from high vacuum

$\circ$ Turn ion gauge off

o Close the high vac valve

- Open vent valve

- Wait for the chamber to reach atmospheric pressure

O Open the chamber

- Pump down from atmosphere to $5 \times 10^{-2}$ Torr

- Put substrate in the chamber as close to the center of the target as possible

- Close the chamber almost all the way

- Close the vent valve

- Close the chamber completely, making sure it lines up to avoid leaks

- Close the foreline valve

- Open the roughing valve (the foreline and roughing valves should never be open at the same time even though the system doesn't have a failsafe to prevent this)

$\circ$ Wait for the pressure to reach at least $5 \times 10^{-2}$ Torr

- Pump from 50mTorr to high vacuum

- Close the roughing valve

- Open the foreline valve

- Wait for the foreline to evacuate (the pressure reading will stabilize)

- Open the hi-vac valve

- Turn on the ion gauge when the chamber is at $10^{-3}$

- Wait for the chamber to reach the desired pressure of $2-3 \times 10^{-6}$ (This can take 2.5-3 hours so you can leave when the pressure is steadily dropping- usually less than $10^{-3}$ )

- $\quad$ Sputtering

○ Turn off the ion gauge

- Turn on the water for head

○ Turn on the power supply 
- Close throttle valve

- Turn on the gas (at the tank and the valve)

- Set gas flow to $10 \mathrm{sccm}$

- Turn on tuning control (this light should normally come on but may not because of a blown fuse)

- Change process to sputter deposit

- Titanium Sputtering

- Need to preclean the titanium target by moving the Table to Target 1 and the target to Target 3 .

- Set the tuning cap positions

- Turn up the power until the plasma lights (reflected power to a little above 10 and press ignition switch).

- Reduce the reflected power $(=0)$ and increase forward power $(=10)$

- Adjust the tuning cap positions as needed to achieve the power settings

- Let run for 5 minutes

- Move the table to Target 3

- Set the tuning cap positions

- Turn up the power until the plasma lights (reflected power to a little above 10 and press ignition switch).

- Reduce the reflected power $(=0)$ and increase forward power $(=10)$

- Adjust the tuning cap positions as needed to achieve the power settings

- Let run for 5 minutes

○ Gold Sputtering

- Doesn't require precleaning, but if you do please preclean only for 30 seconds or so

- Move the table to Target 2

- Set the Target to Target 2

- Set the tuning cap positions

- Turn up the power until the plasma lights (reflected power to a little above 10 and press ignition switch).

- Reduce the reflected power $(=0)$ and increase forward power $(=10)$

- Adjust the tuning cap positions as needed to achieve the power settings

- Let run for desired length of time (deposits at a rate of $20 \mathrm{~nm} / \mathrm{min}$ ) if longer than 5 minutes do 5 minutes on and 2 minute cool down in between each 5 minute period

- Set the table position to "etch"

$\circ$ Turn down the argon flow and switch the valve off (on equipment and at tank)

- Turn off power

- Venting the chamber

○ Make sure the gas is turned off and then open the throttle valve (put in down position)

- Close the hi-vac valve

O Open the vent valve

- Wait for the chamber to reach atmospheric pressure

o Shut vent valve 
- Open the chamber and remove samples

$\circ$ Pump down to hi-vac when finished

\section{E-beam Deposition:}

- Pump up to atmosphere from high vacuum

○ Turn high vacuum gauge off

- Turn knob to manual

- Turn on mechanical pump to allow it time to warn up

- Turn on vent switch

- Release door handle but do not force door open

○ Check temperature- should be 15 Kelvin

- Remove stage by releasing one screw

- Add 3 slides to stage being careful to not put the clip over the design ( 2 design slides and one blank coupon)

- Place stage back in chamber and check each screw to make sure they are tight

- Close door but do not lock

- Turn off vent

- Lock door

- Pump down to high vacuum

- Turn knob to auto pump

- Transition to high vacuum will happen around 120mTorr

- After transition happens turn on high vacuum gauge

- Turn off mechanical pump

- Vent mechanical pump line (switch under high vacuum gauge monitor)

- Deposition

- Turn on power supply and let warm up for 2 minutes

- Turn on deposition rate monitor

- Turn on beam controllers

- Turn on water - both switches are behind chamber

- Turn on rotation

- Select proper channel for metal deposition: 1 is Titanium, 3 is Platinum and 4 is Gold

- Will need a titanium layer as an adhesion layer before depositing platinum or gold

- Rotate crucible holder to proper crucible- 360 degrees rotates holder one position to the right

- Turn on voltage and turn up to operating voltage (written on the white board)

$\circ$ When metal is in evaporative state open the shutter- this starts the deposition process

$\circ$ The deposition rate and thickness are on the deposition monitor so follow these along with the time to determine when to stop deposition (previously have done $50 \mathrm{~nm}$ of titanium, $50 \mathrm{~nm}$ of gold and 10 minutes of platinum)

- Close shutter

- Select proper channel for top deposition level (usually platinum or gold) 
- Rotate crucible holder to proper crucible- 360 degrees rotates holder one position to the right

- Repeat process for this second metal

- Close shutter

- Turn voltage down completely

- Wait 2 minutes before turning off power supply

- Turn off voltage

- Turn off controller

- Turn off water

○ Turn off rotation

- Pump back up to atmospheric pressure

○ Turn off high vacuum gauge

- Turn on mechanical pump to give it time to warm up

- Turn knob to manual

- Unlock door handle

○ Turn on vent

- Check temperature- should be around $15 \mathrm{Kelvin}$

- When vented take out substrate holder and remove samples

- Replace substrate holder, checking all three screws

- Pump down to high vacuum

- Close door but do not lock

- Turn off vent

○ Lock door

- Turn knob to auto pump

$\circ$ Transition to high vacuum will happen around 120mTorr

- After transition happens turn on high vacuum gauge

- Turn off mechanical pump

○ Vent mechanical pump line (switch under high vacuum gauge monitor)

Sonication of Glass Slides:

- Fill clean glass dish with acetone

- Sonicate glass slides in acetone for 10 minutes

- Wash with DI water, rinsing off any excess photoresist and metal

Clean Room Procedure- Silica Wafer:

- Turn hot plates on to $65^{\circ} \mathrm{C}$ and $95^{\circ} \mathrm{C}$

- Setup EVG machine

- Check EVG machine for green light

- Turn on EVG machine at key switch and computer CPU

- Open EVG software

- Install appropriate lithography mask (follow along with computer instructions)

- Important parameters:

- Mask thickness: do not change

- Mask size: 5 inch

- Substrate size: 4 inch 
- Substrate thickness: XX

- Process time: 20 sec (can be optimized based on specific mask)

- Spin coat silica wafer with SU-8 2025

○ Spin at 750rpm for $15 \mathrm{sec}$ with accl $=100$

- Spin at $1500 \mathrm{rpm}$ for $30 \mathrm{sec}$ with accl=300

- Pre-bake at:

- $65^{\circ} \mathrm{C}$ for 3 minutes

- $95^{\circ} \mathrm{C}$ for 6 minutes

- Expose to UV light for appropriate amount of time

- Post-bake at:

- $65^{\circ} \mathrm{C}$ for 6 minutes

- $95^{\circ} \mathrm{C}$ for 12 minutes

- Soak in SU-8 developer for 10 minutes

○ Stop every 3 minutes or so and wash wafer off with IPA

- Wash with IPA

- Dry with Nitrogen

Fabrication of Devices:

- Put glass slide and PDMS casting in plasma generator

- Pump down for 2 minutes

- Expose to plasma for 30 seconds on RF level of medium

- Turn off plasma generation and pump

- Immediately bring generator back up to room pressure

- Immediately bring glass slide and PDMS casting in contact with each other, lining up electrodes and chamber

- Put device in oven at $60^{\circ} \mathrm{C}$ for at least 3 hours

- Test sealing of device by injecting E-pure water into device and checking for any leaks

- Add copper electrodes with silver epoxy and bonded port connector with regular epoxy

- Put device back in oven for at least an hour at $95^{\circ} \mathrm{C}$ 


\subsection{Matlab Code}

\subsubsection{Theory Code:}

$\%$ Kaela M. Leonard

\% Michigan Technological University

$\%$ October 26th, 2010

$\%$ April 1st, 2012

$\%$ May 28th, 2012

$\%$ May 30th, 2012 - finally fixed the problems with the shelled ellipsoid

$\%$ major axis

$\%$ This program is used to calculate the Clausius-Mossotti factor based on $\%$ multiple different models, focusing on the shelled ellipsoid model and $\%$ using three different medium conductivities.

clear all

clc

$\%$ Parameter definitions

$\mathrm{e} 0=8.85^{*} 10^{\wedge}-12 ; \quad \%$ Permittivity of a vacuum $(\mathrm{F} / \mathrm{m})$

\% Erythrocyte dielectric parameters taken from Gascoyne, P. Acta Tropica 89

$\%$ (2004) 


$$
\begin{array}{lr}
\text { ec }=59 * \mathrm{e} 0 ; & \% \text { Permittivity of particle core }(\mathrm{F} / \mathrm{m}) \\
\mathrm{sc}=.31 ; & \% \text { Conductivity of particle core }(\mathrm{S} / \mathrm{m}) \\
\mathrm{es}=4.44 * \mathrm{e} 0 ; & \% \text { Permittivity of particle shell }(\mathrm{F} / \mathrm{m}) \\
\mathrm{ss}=10^{\wedge}-7 ; & \% \text { Conductivity of particle shell }(\mathrm{S} / \mathrm{m})
\end{array}
$$

\%Medium permittivity taken from Wang, M.H. Biosensors and Bioelectronics 24 $\%$ (2009) for their PBS buffer

$$
\text { em }=136 * \mathrm{e} 0 ; \quad \% \text { Permittivity of medium }(\mathrm{F} / \mathrm{m})
$$

$\%$ Medium conductivity taken from experimental results $\mathrm{sm}=[0.01,0.1,0.9] ; \quad \%$ Conductivity of medium $(\mathrm{S} / \mathrm{m})$ $[\mathrm{M}, \mathrm{N}]=\operatorname{size}(\mathrm{sm})$

freq $=[10000: 1000: 100000000] ; \quad \%$ Frequency $(\mathrm{Hz})$ $\mathrm{W}=(2 * \mathrm{pi})$. freq; $\quad \% O m e g a$

\%Erythrocyte size parameters taken from Pallister Blood: Physiology and \%Pathophysiology, 1994.

$$
\begin{array}{ll}
\mathrm{a}=3.5^{*} 10^{\wedge}-6 ; & \text { \%Radius of long edge }(\mathrm{m}) \\
\mathrm{c}=1 * 10^{\wedge}-6 ; & \text { \%Radius of short edge }(\mathrm{m}) \\
\mathrm{d}=4.6^{*} 10^{\wedge}-9 ; & \text { \%Thickness of membrane }(\mathrm{m})
\end{array}
$$

$\%$ Calculation of Depolarization Factor 
$1=[0$ inf $]$

$\mathrm{b}=\left(\mathrm{a}^{\wedge} 2\right)-\left(\mathrm{c}^{\wedge} 2\right)$

$\mathrm{f}=\left(\mathrm{c}^{\wedge} 2\right)+1$

$\mathrm{j}=\left(\mathrm{a}^{\wedge} 2\right)+1$

inta $=\left(\left(\operatorname{sqrt}\left(\left(\mathrm{c}^{\wedge} 2\right)+1\right) \cdot /\left(\left(\left(\mathrm{a}^{\wedge} 2\right)-\left(\mathrm{c}^{\wedge} 2\right)\right) \cdot *\left(\left(\mathrm{a}^{\wedge} 2\right)+1\right)\right)\right)+\left(\left(\operatorname{atan}\left(\left(\operatorname{sqrt}\left(\left(\mathrm{c}^{\wedge} 2\right)+1\right)\right) \cdot /\left(\operatorname{sqrt}\left(\left(\mathrm{a}^{\wedge} 2\right)-\right.\right.\right.\right.\right.\right.$

$\left.\left.\left.\left.\left.\left.\left(\mathrm{c}^{\wedge} 2\right)\right)\right)\right)\right) /\left(\left(\left(\mathrm{a}^{\wedge} 2\right)-\left(\mathrm{c}^{\wedge} 2\right)\right)^{\wedge}(3 / 2)\right)\right)\right)$;

syms y; \%Assigns a symbolic variable y to be used in the below computation

$\mathrm{x}=\operatorname{limit}\left(\left(\left(\operatorname{sqrt}\left(\left(\mathrm{c}^{\wedge} 2\right)+\mathrm{y}\right) . /\left(\left(\left(\mathrm{a}^{\wedge} 2\right)-\left(\mathrm{c}^{\wedge} 2\right)\right) \cdot *\left(\left(\mathrm{a}^{\wedge} 2\right)+\mathrm{y}\right)\right)\right)+\left(\left(\operatorname{atan}\left(\left(\operatorname{sqrt}\left(\left(\mathrm{c}^{\wedge} 2\right)+\mathrm{y}\right)\right) . /\left(\operatorname{sqrt}\left(\left(\mathrm{a}^{\wedge} 2\right)-\right.\right.\right.\right.\right.\right.\right.$ $\left.\left.\left.\left.\left.\left.\left(\mathrm{c}^{\wedge} 2\right)\right)\right)\right)\right) /\left(\left(\left(\mathrm{a}^{\wedge} 2\right)-\left(\mathrm{c}^{\wedge} 2\right)\right)^{\wedge}(3 / 2)\right)\right)\right), \mathrm{y}$, inf $) ; \%$ Calculates $\mathrm{x}$ which is the limit as y approaches infinity of the expression for inta

$\mathrm{x}=$ double $(\mathrm{x})$; \% Switches $\mathrm{x}$ from being a symbolic variable to a double variable (which for matlab is a numerical variable)

$\operatorname{intc}=(2 . /((0-b) \cdot *(\operatorname{sqrt}(f))))-\left((2 \cdot *(\operatorname{atan}((\operatorname{sqrt}(f)) \cdot /(\operatorname{sqrt}(b))))) \cdot /\left(b^{\wedge}(3 / 2)\right)\right)$

constant $=(\mathrm{a} * \mathrm{a} * \mathrm{c}) / 2$

$\mathrm{La}=$ constant $*(\mathrm{x}-\mathrm{inta}(1)) ; \%$ Depolarization factor for major axis

$\mathrm{Lc}=$ constant $*(\operatorname{intc}(2)-\operatorname{intc}(1)) ; \%$ Depolarization factor for minor axis

for $\mathrm{i}=1: \mathrm{N}$;

$\%$ Calculation of Complex Permittivities

$$
\begin{aligned}
& \mathrm{cec}=\mathrm{ec}+\left(\mathrm{sc} . /\left((\operatorname{sqrt}(-1)) .{ }^{*} \mathrm{w}\right)\right) ; \quad \% \text { Complex permittivity of particle core } \\
& \text { ces }=\text { es }+\left(\operatorname{ss} . /\left((\operatorname{sqrt}(-1)) .{ }^{*} \mathrm{w}\right)\right) ; \quad \% \text { Complex permittivity of particle shell } \\
& \operatorname{cem}(\mathrm{i},:)=\mathrm{em}+\left(\operatorname{sm}(\mathrm{i}) \cdot /\left((\operatorname{sqrt}(-1)) \cdot{ }^{*} \mathrm{w}\right)\right) ; \quad \% \text { Complex permittivity of medium } \\
& \mathrm{g}=\left(\operatorname{cec} .{ }^{*} \operatorname{ces}\right) \cdot /\left(\left((\mathrm{d} / \mathrm{a}) \cdot{ }^{*} \text { cec }\right)+\text { ces }\right) \\
& \mathrm{h}=(\text { cec. } * \text { ces }) . /((\mathrm{d} / \mathrm{c}) \cdot * \text { cec })+\mathrm{ces})
\end{aligned}
$$


$\%$ Calculation of Sphere Model

FCMs $(\mathrm{i},:)=(\operatorname{cec}-\operatorname{cem}(\mathrm{i},:)) . /\left(\operatorname{cec}+\left(2{ }^{*} \operatorname{cem}(\mathrm{i},:)\right)\right) ; \quad \%$ Clausius-Mossotti factor for a spherical model

RealFCMs $=\operatorname{real}(\mathrm{FCMs}) ; \quad \%$ Real part of Clausius-Mossotti factor

$\%$ Calculation of Shelled Sphere Model

FCMss $(\mathrm{i},:)=\left(\left(\left(\right.\right.\right.$ cec. ${ }^{*}$ ces $\left.\left.) . /\left(\left((\mathrm{d} / \mathrm{a}) .{ }^{*} \operatorname{cec}\right)+\operatorname{ces}\right)\right)-\operatorname{cem}(\mathrm{i},:)\right) . /\left(\left(\left(\mathrm{cec}{ }^{*}{ }^{*} \operatorname{ces}\right) . /\left(\left((\mathrm{d} / \mathrm{a}) .{ }^{*} \operatorname{cec}\right)+\right.\right.\right.$ ces $\left.))+\left(2 \cdot{ }^{*} \operatorname{cem}(\mathrm{i},:)\right)\right)$;

\%Clausius-Mossotti factor for a shelled spherical model

RealFCMss $=\operatorname{real}(\mathrm{FCMss}) ; \quad$ \%Real part of Clausius-Mossotti factor

$\%$ Calculation of Shelled Ellipsoid Model

$\operatorname{diffa}(\mathrm{i},:)=\mathrm{g}-\operatorname{cem}(\mathrm{i},:)$;

$\operatorname{diffc}(\mathrm{i},:)=\mathrm{h}-\operatorname{cem}(\mathrm{i},:)$;

FCMa $(\mathrm{i},:)=\operatorname{diffa}(\mathrm{i},:) . /\left(3 . *\left(\operatorname{cem}(\mathrm{i},:)+\left(\right.\right.\right.$ La. $\left.\left.\left.{ }^{*} \operatorname{diffa}(\mathrm{i},:)\right)\right)\right) ; \%$ Clausius-Mossotti factor for major axis

$\operatorname{FCMc}(i,:)=\operatorname{diffc}(i,:) . /(3 . *(\operatorname{cem}(i,:)+(\operatorname{Lc} . * \operatorname{diffc}(i,:)))) ; \%$ Clausius-Mossotti factor for minor axis

RealFCMa=real(FCMa); $\quad \%$ Real part of Clausius-Mossotti factor for major axis

RealFCMc $=$ real $(\mathrm{FCMc}) ; \quad \%$ Real part of Clausius-Mossotti factor for minor axis

end

\%This section of code tries to calculate a complete Clausius-Mossotti 
\%factor for the ellipsoid model based on the idea that the major and minor $\%$ axis act as two parts of a triangle. Not working perfectly.

magnitude $=\operatorname{sqrt}\left(\left((\right.\right.$ RealFCMa $\left.) .^{\wedge} 2\right)+(($ RealFCMc $\left.) . \wedge 2)\right)$

theta $=\operatorname{atan}($ RealFCMa./RealFCMc $)$;

[rows columns]=size(freq);

$\mathrm{x}=\operatorname{zeros}(3$, columns $)$;

for $m=1: 3$

for $\mathrm{i}=1$ : columns

if $($ theta $(m, i)<=$ pi $) \& \&(\operatorname{theta}(m, i)>=0)$

$\mathrm{x}(\mathrm{m}, \mathrm{i})=1$;

else

$$
\mathrm{x}(\mathrm{m}, \mathrm{i})=-1 \text {; }
$$

end

end

end

RealFCM = magnitude. ${ }^{*} \mathrm{x}$;

\%Plotting of Real Parts of Clausius-Mossotti Factors

figure

semilogx(freq, RealFCMs(1,:), 'r', freq, RealFCMs(2,:), 'g', freq, RealFCMs(3,:), 'b', 'LineWidth', 4)

\% title('Frequency vs. Real Part of the Clausius Mossotti Factor for Spherical Model')

$\%$ xlabel('Frequency')

\% ylabel('Real Part of the Clausius Mossotti Factor') 
$\%$ legend('0.01S/m Medium Conductivity', '0.1S/m Medium Conductivity', '0.9S/m Medium Conductivity')

figure

semilogx(freq, RealFCMss(1,:), 'r', freq, RealFCMss(2,:), 'g', freq, RealFCMss(3,:), 'b', 'LineWidth', 4)

\% title('Frequency vs. Real Part of the Clausius Mossotti Factor for Shelled Spherical Model')

$\%$ xlabel('Frequency')

$\%$ ylabel('Real Part of the Clausius Mossotti Factor')

$\%$ legend('0.01S/m Medium Conductivity', '0.1S/m Medium Conductivity', '0.9S/m Medium Conductivity')

figure

semilogx(freq, RealFCMc(1,:), 'r', freq, RealFCMc(2,:), 'g', freq, RealFCMc(3,:), 'b', 'LineWidth', 4)

\% title('Frequency vs. Real Part of the Clausius Mossotti Factor for Shelled Ellipsoidal Model- Minor Axis')

$\%$ xlabel('Frequency')

$\%$ ylabel('Real Part of the Clausius Mossotti Factor')

$\%$ legend('0.01S/m Medium Conductivity', '0.1S/m Medium Conductivity', '0.9S/m Medium Conductivity')

figure

semilogx(freq, RealFCMa(1,:), 'r', freq, RealFCMa(2,:), 'g', freq, RealFCMa(3,:), 'b', 'LineWidth', 4)

\% title('Frequency vs. Real Part of the Clausius Mossotti Factor for Shelled Ellipsoidal Model- Major Axis')

$\%$ xlabel('Frequency')

\% ylabel('Real Part of the Clausius Mossotti Factor') 
$\%$ legend('0.01S/m Medium Conductivity', '0.1S/m Medium Conductivity', '0.9S/m Medium Conductivity')

figure

semilogx(freq, RealFCMa(1,:), 'r-', freq, RealFCMc(1,:), 'r-.', 'LineWidth', 4)

\% title('Frequency vs. Real Part of the Clausius Mossotti Factor for Shelled Ellipsoidal Model at $0.01 \mathrm{~S} / \mathrm{m}^{\prime}$ )

$\%$ xlabel('Frequency')

\% ylabel('Real Part of the Clausius Mossotti Factor')

\% legend('Major Axis', 'Minor Axis')

figure

semilogx(freq, RealFCMa(2,:), 'g-', freq, RealFCMc(2,:), 'g-.', 'LineWidth', 4)

\% title('Frequency vs. Real Part of the Clausius Mossotti Factor for Shelled Ellipsoidal Model at $\left.0.1 \mathrm{~S} / \mathrm{m}^{\prime}\right)$

$\%$ xlabel('Frequency')

\% ylabel('Real Part of the Clausius Mossotti Factor')

\% legend('Major Axis', 'Minor Axis')

figure

semilogx(freq, RealFCMa(3,:), 'b-', freq, RealFCMc(3,:), 'b-.', 'LineWidth', 4)

$\%$ title('Frequency vs. Real Part of the Clausius Mossotti Factor for Shelled Ellipsoidal Model at $\left.0.9 \mathrm{~S} / \mathrm{m}^{\prime}\right)$

$\%$ xlabel('Frequency')

\% ylabel('Real Part of the Clausius Mossotti Factor')

$\%$ legend('Major Axis', 'Minor Axis')

figure

semilogx(freq, RealFCM(1,:), 'r', freq, RealFCM(2,:), 'g', freq, RealFCM(3,:), 'b', 'LineWidth', 4) 
\% title('Frequency vs. Real Part of the Clausius Mossotti Factor for Shelled Ellipsoidal Model- Combined')

$\%$ xlabel('Frequency')

$\%$ ylabel('Real Part of the Clausius Mossotti Factor')

$\%$ legend('0.01S/m Medium Conductivity', '0.1S/m Medium Conductivity', '0.9S/m Medium Conductivity')

\subsubsection{Main Code:}

$\%$ Kaela M. Leonard

\%Thursday, July 12th, 2012

$\%$ Thursday, July 26th, 2012

$\%$ This code takes allows the user to run multiple sweeps through the program

$\%$ so that all the sweeps for a given experiment can be run at once.

clear all

clc

$\mathrm{i}=$ input('How many files would you like to analyze?');

$\%$ This for loop takes the function "profiles" and runs it for each sweep of

$\%$ interest. It then takes the Combined Intensity Data (not scaled) along

$\%$ with the maximum and minimum values of this Combined Intensity and puts

$\%$ them in matrices. The end result is a matrix of all the combined

$\%$ intensity data for all the sweeps and two vectors- one of the maximum

$\%$ intensity value for each sweep and one of the minimum intensity value for

$\%$ each sweep. It also pulls out the frequency data for each sweep.

freq $=$ zeros $(402, \mathrm{i})$;

AllCombined=zeros $(402, \mathrm{i})$;

for $\mathrm{z}=1: \mathrm{i}$

file1=input('What is the file name of the TL to BR file (including .xml at the end)?','s');

file2=input('What is the file name of the BL to TR file (including .xml at the end)?','s');

file3=input('What is the file name of the Horizontal file (including .xml at the end)?','s');

file4=input('What is the file name (including .xml at the end) of the Vertical file?', 's');

file $5=\operatorname{input}($ 'What is the file name (including .xml at the end) of the background file?', 's');

$\mathrm{SF}=$ input('What is the starting frequency in $\mathrm{MHz}$ ?');

$\mathrm{EF}=$ input('What is the ending frequency in $\mathrm{MHz}$ ?');

$\mathrm{T}=$ input('How many seconds did the sweep last for?');

[freqtemp combined AllMax(z) AllMin(z)]= profilesNewAvgNonMoving(file1, file2, file3, file4, file5, SF, EF, T);

$\mathrm{b}=\operatorname{size}($ combined);

for $s=1: b(2)$ 


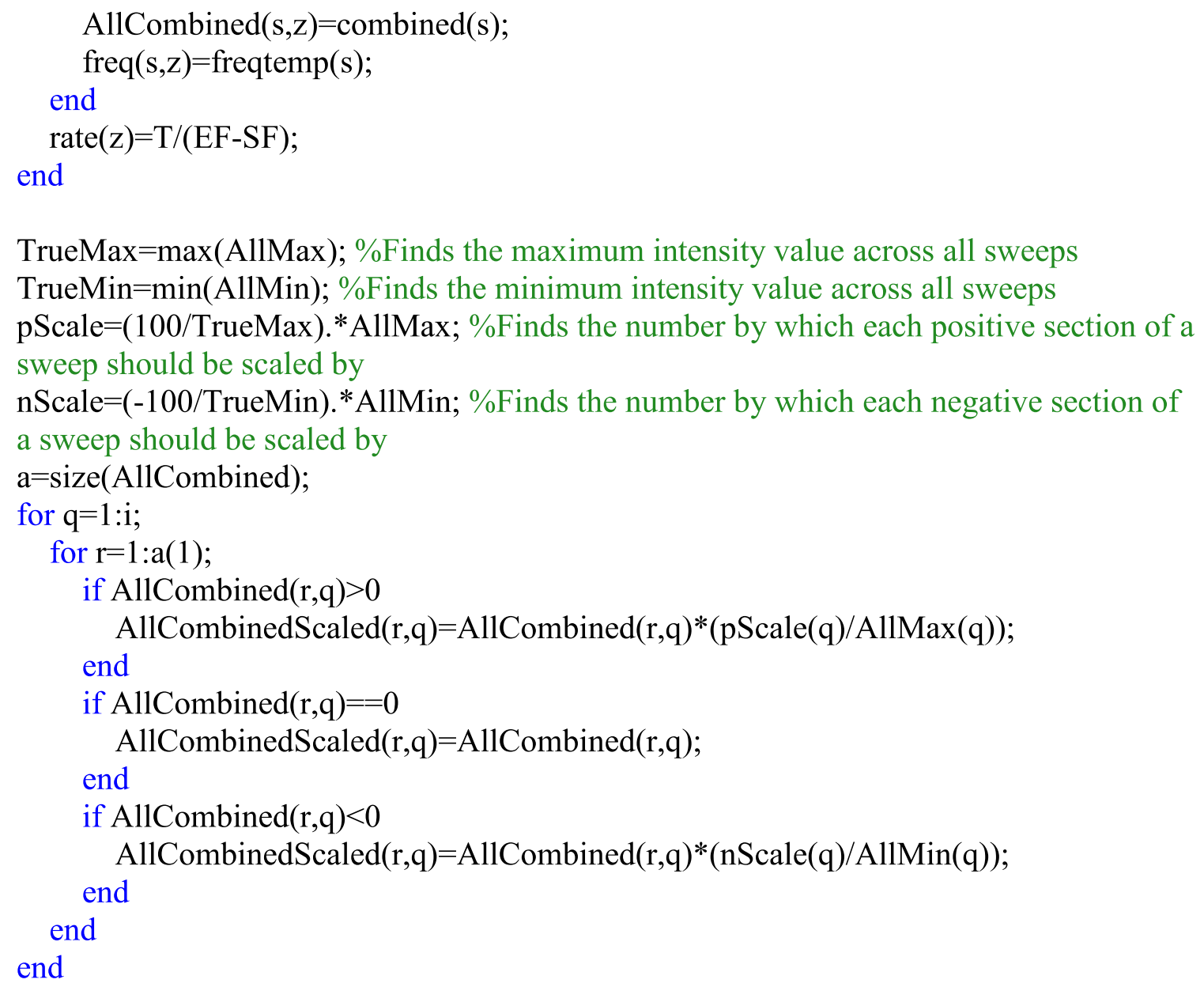


\%Plots:

figure

plot(freq, AllCombined, '^', freq, AllCombinedScaled, '*', 'LineWidth', 4)

axis auto

legend('Sweep 1', 'Sweep 2', 'Sweep 3', 'Sweep 4', 'Sweep 5', 'Sweep 6', 'Sweep 1 Scaled', 'Sweep 2 Scaled', 'Sweep 3 Scaled', 'Sweep 4 Scaled', 'Sweep 5 Scaled', 'Sweep 6

Scaled')

figure

plot(FreqEvened, CombinedEvened, '*', 'LineWidth', 4)

axis auto

legend('Sweep 1 Scaled', 'Sweep 2 Scaled', 'Sweep 3 Scaled', 'Sweep 4 Scaled', 'Sweep 5 Scaled', 'Sweep 6 Scaled') 


\subsubsection{Function}

\section{function [freq combinedintb maxcombined mincombined] $=$ profilesNewAvgNonMoving(file1, file2, file3, file4, file5, SF, EF, T)}

$\%$ Kaela M. Leonard

\%Monday, April 2nd, 2012

$\%$ Wednesday, April 4th, 2012

\%Thursday, April 5th, 2012

\%Monday, April 30th, 2012

\%Monday, June 25th, 2012

\%Tuesday, June 26th, 2012

$\%$ Tuesday, July 10th, 2012

$\%$ Wednesday, July 11 th, 2012

\%Thursday, July 12th, 2012

\%Friday, July 13th, 2012

\%Saturday, July 14th, 2012

\%Thursday, July 26th, 2012

$\%$ Function analyzes profiles from COF sweep videos and combines this with a

$\%$ bias value that ranges from -1 (strong $\mathrm{nDEP}$ ) to +1 (strong $\mathrm{pDEP}$ ) in order

$\%$ to get an overall qualitative assement of the DEP force felt by the

$\%$ particles at each frequency. This uses the intesity profile with the

$\%$ background intensity subtracted and uses a combined step function and

$\%$ linear bias for intensity.

\%Anything with a 1 after it refers to TL to BR intensity profile

$\%$ Anything with a 2 after it refers to BL to TR intensity profile

$\%$ Anything with a 3 after it refers to Horizontal intensity profile

$\%$ Anything with a 4 after it refers to Vertical intensity profile

$\%$ Anything with a 5 after it refers to Background intensity profile

$\%$ clear all

$\%$ clc

$\%$

$\%$ file $1=$ input('What is the file name of the TL to BR file (including .xml at the end)?','s');

$\%$ file $2=$ input('What is the file name of the BL to TR file (including .xml at the end)?','s');

$\%$ file $3=$ input('What is the file name of the Horizontal file (including .xml at the

end)?','s');

$\%$ file4=input('What is the file name (including .xml at the end) of the Vertical file?', 's');

$\%$ file $5=$ input('What is the file name (including .xml at the end) of the background file?',

's');

$\% \mathrm{SF}=$ input('What is the starting frequency in $\mathrm{MHz}$ ');

$\% \mathrm{EF}=\operatorname{input}($ 'What is the ending frequency in $\mathrm{MHz}$ ');

$\% \mathrm{~T}=$ input('How many seconds did the sweep last for?');

\% This section reads the TL to BR file and breaks up the matrix into 
\%Position Number, $\mathrm{x}$ data, $\mathrm{y}$ data and intensity data matrices

[ndata1, tdata1, alldata1] =xlsread(file1); \% This line reads in a xls or xml file- in this case the profile of interest

$[\mathrm{a} 1 \mathrm{~b} 1]=\operatorname{size}($ ndata 1$) ; \%$ This line assigns a as the number of rows and $\mathrm{b}$ as the number of columns in the profile matrix

PosNum1=ndata1(:,1); \% This line assign PosNum as a new column matrix consisting of column 1 of the original profile matrix

$\mathrm{x} 1=$ ndata1(:,2); \% This line assign $\mathrm{x}$ as a new column matrix consisting of column 2 of the original profile matrix- $\mathrm{x}$ positions

$\mathrm{y} 1=$ ndata1(:,3); \% This line assign y as a new column matrix consisting of column 3 of the original profile matrix- $y$ positions

for $\mathrm{k}=4: 1: \mathrm{b} 1$

$\operatorname{int1}(:, \mathrm{k}-3)=$ ndata1 $(:, \mathrm{k}) ; \%$ This for loop allocates an intensity matrix to take the intensity data from the original profile matrix

end

\% This section reads the BL to TR file and breaks up the matrix into

$\%$ Position Number, $\mathrm{x}$ data, $\mathrm{y}$ data and intensity data matrices

[ndata2, tdata2, alldata2] $=x 1 s r e a d(f i l e 2) ; \%$ This line reads in a xls or xml file- in this case the profile of interest

$[\mathrm{a} 2 \mathrm{~b} 2]=\operatorname{size}($ ndata2); \% This line assigns a as the number of rows and $\mathrm{b}$ as the number of columns in the profile matrix

PosNum2=ndata2(:,1); \% This line assign PosNum as a new column matrix consisting of column 1 of the original profile matrix

$\mathrm{x} 2=$ ndata2(:,2); \% This line assign $\mathrm{x}$ as a new column matrix consisting of column 2 of the original profile matrix- $\mathrm{x}$ positions

$\mathrm{y} 2=$ ndata2(:,3); \% This line assign y as a new column matrix consisting of column 3 of

the original profile matrix-y positions

for $\mathrm{k}=4: 1: \mathrm{b} 2$

int2(:,k-3)=ndata2(:,k); \% This for loop allocates an intensity matrix to take the intensity data from the original profile matrix

end

\% This section reads the Horizontal file and breaks up the matrix into

$\%$ Position Number, $\mathrm{x}$ data, $\mathrm{y}$ data and intensity data matrices

[ndata3, tdata3, alldata3]=xlsread(file3); \% This line reads in a xls or xml file- in this case the profile of interest

[a3 b3]= size(ndata3); \% This line assigns a as the number of rows and $b$ as the number of columns in the profile matrix

PosNum3=ndata3(:,1); \% This line assign PosNum as a new column matrix consisting of column 1 of the original profile matrix

$\mathrm{x} 3=$ ndata3(:,2); \% This line assign $\mathrm{x}$ as a new column matrix consisting of column 2 of the original profile matrix- $\mathrm{x}$ positions

$\mathrm{y} 3=$ ndata $3(:, 3) ; \%$ This line assign $\mathrm{y}$ as a new column matrix consisting of column 3 of the original profile matrix-y positions

for $\mathrm{k}=4: 1: \mathrm{b} 3$ 
int3(:,k-3)=ndata3(:,k); \% This for loop allocates an intensity matrix to take the intensity data from the original profile matrix

end

\% This section reads the Vertical file and breaks up the matrix into

$\%$ Position Number, $\mathrm{x}$ data, $\mathrm{y}$ data and intensity data matrices

[ndata4, tdata4, alldata4]=xlsread(file4); \% This line reads in a xls or xml file- in this case the profile of interest

$[\mathrm{a} 4 \mathrm{~b} 4]=\operatorname{size}($ ndata4); \% This line assigns a as the number of rows and $\mathrm{b}$ as the number of columns in the profile matrix

PosNum4=ndata4(:,1); \% This line assign PosNum as a new column matrix consisting of column 1 of the original profile matrix

$\mathrm{x} 4=$ ndata4(:,2); \% This line assign $\mathrm{x}$ as a new column matrix consisting of column 2 of the original profile matrix- $\mathrm{x}$ positions

y4=ndata4(:,3); \% This line assign y as a new column matrix consisting of column 3 of the original profile matrix- y positions

for $\mathrm{k}=4: 1: \mathrm{b} 4$

$\operatorname{int} 4(:, \mathrm{k}-3)=$ ndata4 $(:, \mathrm{k}) ; \%$ This for loop allocates an intensity matrix to take the intensity data from the original profile matrix

end

\% This section takes the background file and calculates the average background

$\%$ intensity

$[$ ndata5, tdata5, alldata5] $=x$ lsread(file5); \% This line reads in a xls or xml file- in this case the profile of interest

$[\mathrm{cd}]=\operatorname{size}($ ndata5); \% This line assigns a as the number of rows and $\mathrm{b}$ as the number of columns in the profile matrix

intb=ndata5(:,4); \% This for loop allocates an intensity matrix to take the intensity data from the original profile matrix

avgintb=mean(intb); \%This line gets the mean value of the background intensity matrix

$\%$ This section creates a frequency matrix for the sweep of interest

freq $=$ zeros $(\mathrm{b} 2-4,1)$;

time $=$ zeros $(\mathrm{b} 2-4,1)$;

for $m=1: 1: b 2-4$

time $(\mathrm{m}+1)=\operatorname{time}(\mathrm{m})+(\mathrm{T} /(\mathrm{T}+1)) ; \%$ This assign a time column matrix based on the length of time the sweep was run for

$\operatorname{freq}(1)=0$;

freq $(\mathrm{m}+1)=(((\mathrm{EF}-\mathrm{SF}) /(\mathrm{T}-1)) *$ time $(\mathrm{m}+1))+\mathrm{SF} ; \%$ This assigns a frequency column matrix based on the length of time of the sweep and the start and end frequencies end

$\%$ This sections checks to see if the intensity of a given position number $\%$ doesn't change across the entire time range 
\%TL to BR Profile:

inttranspose1=int1'; \% This line transposes the intensity matrix so that the standard deviation across time can be taken

[e1 fl] $=$ size(int1); \% This line takes the size of the original matrix where the number of columns is the number of time points and the number of rows is the number of positions stdevint $1=$ std(inttranspose 1$) ; \%$ This line takes the standard deviation of each position across all timepoints

stderror1=stdevint1./(f1); \% This line finds the standard error by dividing the standard deviation by the number of points that went into it (aka the number of time points) erroradjint $1=$ zeros $(\mathrm{e} 1, \mathrm{fl}) ; \%$ This line allocates an empty matrix that is the same size as the original intensity matrix

for $\mathrm{q} 1=1: \mathrm{e} 1 ; \%$ This for loop goes through the entire range of positions

if stderror $1(\mathrm{q} 1)<0.5$; \% This if loop checks to see if the standard deviation of a given position is less than a set acceptable value

erroradjint1(q1,:)=avgintb; \%If the standard error is less than acceptable then all intensity values in that row drop to being equal to the average background intensity end

if stderror1(q1) $>0.5$; \% This if loop checks to see if the standard deviation of a given position is greater than a set acceptable value

erroradjint1(q1,:)=int1(q1,:); \% If the standard error is greater than acceptable then all intensity values in that row are set equal to their original intensity values

end

end

$\%$ BL to TR Profile:

inttranspose2=int2'; \% This line transposes the intensity matrix so that the standard deviation across time can be taken

[e2 f2] $=$ size(int2); \% This line takes the size of the original matrix where the number of columns is the number of time points and the number of rows is the number of positions stdevint $2=\operatorname{std}($ inttranspose 2$) ; \%$ This line takes the standard deviation of each position across all timepoints

stderror2=stdevint2./(f2); \%This line finds the standard error by dividing the standard deviation by the number of points that went into it (aka the number of time points) erroradjint $2=$ zeros $(e 2, f 2) ; \%$ This line allocates an empty matrix that is the same size as the original intensity matrix

for $\mathrm{q} 2=1: \mathrm{e} 2 ; \%$ This for loop goes through the entire range of positions

if stderror2(q2)<0.5; \% This if loop checks to see if the standard deviation of a given position is less than a set acceptable value

erroradjint $2(\mathrm{q} 2,:)=$ avgintb; \%If the standard error is less than acceptable then all intensity values in that row drop to being equal to the average background intensity end

if stderror2(q2) $>0.5 ; \%$ This if loop checks to see if the standard deviation of a given position is greater than a set acceptable value

erroradjint2(q2,:)=int2(q2,:); \% If the standard error is greater than acceptable then all intensity values in that row are set equal to their original intensity values end 
end

\%Horizontal Profile:

inttranspose 3=int3'; \% This line transposes the intensity matrix so that the standard deviation across time can be taken

[e3 f3] =size(int3); \% This line takes the size of the original matrix where the number of columns is the number of time points and the number of rows is the number of positions stdevint3=std(inttranspose 3); \% This line takes the standard deviation of each position across all timepoints

stderror3=stdevint3./(f3); \%This line finds the standard error by dividing the standard deviation by the number of points that went into it (aka the number of time points)

erroradjint $3=$ zeros $(e 3, f 3) ; \%$ This line allocates an empty matrix that is the same size as the original intensity matrix

for $\mathrm{q} 3=1: \mathrm{e} 3$; \% This for loop goes through the entire range of positions

if stderror $3(\mathrm{q} 3)<0.5 ; \%$ This if loop checks to see if the standard deviation of a given position is less than a set acceptable value

erroradjint3(q3,:)=avgintb; \%If the standard error is less than acceptable then all intensity values in that row drop to being equal to the average background intensity end

if stderror3(q3)>0.5; \% This if loop checks to see if the standard deviation of a given position is greater than a set acceptable value

erroradjint3(q3,:)=int3(q3,:); \%If the standard error is greater than acceptable then all intensity values in that row are set equal to their original intensity values end end

$\%$ Vertical Profile:

inttranspose4=int4'; \% This line transposes the intensity matrix so that the standard deviation across time can be taken

[e4 f4]=size(int4); \% This line takes the size of the original matrix where the number of columns is the number of time points and the number of rows is the number of positions stdevint4=std(inttranspose4); \% This line takes the standard deviation of each position across all timepoints

stderror4=stdevint4./(f4); \% This line finds the standard error by dividing the standard deviation by the number of points that went into it (aka the number of time points)

erroradjint $4=$ zeros $(\mathrm{e} 4, \mathrm{f} 4) ; \%$ This line allocates an empty matrix that is the same size as the original intensity matrix

for $\mathrm{q} 4=1: \mathrm{e} 4 ; \%$ This for loop goes through the entire range of positions

if stderror4(q4)<0.5; \% This if loop checks to see if the standard deviation of a given position is less than a set acceptable value

erroradjint4(q4,:)=avgintb; \%If the standard error is less than acceptable then all intensity values in that row drop to being equal to the average background intensity

end

if stderror4(q4) $>0.5$; \% This if loop checks to see if the standard deviation of a given position is greater than a set acceptable value 
erroradjint4(q4,:)=int4(q4,:); \%If the standard error is greater than acceptable then all intensity values in that row are set equal to their original intensity values end end

\% This section subtracts the background intesity from the main intensity $\%$ matrix and takes the absolute value of this to use as the main intensity $\%$ value $\%$ TL to BR Profile:

inttemp1=erroradjint1-avgintb; \%This line subtracts the average background intensity from the intensity adjusted to account for rows that don't change intensity intabs1=abs(inttemp1); \% This line comes up with the final intensity matrix that is used in all later calculations $\% \mathrm{BL}$ to TR Profile: inttemp2=erroradjint2-avgintb; \%This line subtracts the average background intensity from the intensity adjusted to account for rows that don't change intensity intabs2=abs(inttemp2); \% This line comes up with the final intensity matrix that is used in all later calculations

$\%$ Horizontal Profile:

inttemp3=erroradjint3-avgintb; \%This line subtracts the average background intensity from the intensity adjusted to account for rows that don't change intensity intabs3=abs(inttemp3); \% This line comes up with the final intensity matrix that is used in all later calculations

$\%$ Vertical Profile:

inttemp4=erroradjint4-avgintb; \%This line subtracts the average background intensity from the intensity adjusted to account for rows that don't change intensity intabs4=abs(inttemp4); \% This line comes up with the final intensity matrix that is used in all later calculations

\%This section calculates the bias value for each xscale value based on a $\%$ linear profile of the bias where close to the electrodes has a value of +1 $\%$ and near the center of the profile line has a value of -1 .

\%TL to BR Profile:

PosNumMed1 =median(PosNum1); \% This line determines the median position number $\mathrm{j} 1=\operatorname{round}(\operatorname{PosNumMed} 1) ; \%$ The rounded midpoint of the position number

$\mathrm{f} 1=\mathrm{j} 1-20 ; \%$ The beginning of the flat middle region

$\mathrm{g} 1=\mathrm{j} 1+20 ; \%$ The end of the flat middle region

$\mathrm{e} 1=$ PosNum1(21); \% The end of the first flat region

$\mathrm{h} 1=$ PosNum1(a1-20); \% The beginning of the last flat region

$\%$ These for loops make the twenty pixels closest to the electrodes have a bias

$\%$ value of +1

for $\mathrm{i}=1: \mathrm{g} 1$;

bias1(i)=1; 
end

for $\mathrm{i}=\mathrm{h} 1: \operatorname{PosNum} 1(\mathrm{a} 1)$; bias1(i)=1;

end

$\%$ This for loop makes the twenty pixels in the center of the profile line

$\%$ have a bias value of -1

for $\mathrm{i}=\mathrm{fl}: \mathrm{g} 1$;

$\operatorname{bias} 1(\mathrm{i})=-1$;

end

$\%$ These for loops make the two leftover regions have a linear bias that goes

$\%$ from +1 near the electrodes to -1 near the center

$\%$ First region of linear decay from +1 to -1

for $\mathrm{i}=\mathrm{e} 1: \mathrm{fl}$;

$\operatorname{bias} 1(\mathrm{i})=((-2 /(\mathrm{fl}-\mathrm{e} 1)) *(\operatorname{PosNum} 1(\mathrm{i})-\mathrm{PosNum} 1(\mathrm{e} 1)))+1$;

end

$\%$ Second region of linear increase from -1 to +1

for $\mathrm{i}=\mathrm{g} 1: \mathrm{h} 1$;

bias1(i) $=((2 /($ h1-g1) $) *($ PosNum1(i)-PosNum1(h1)) $)+1$;

end

$\% \mathrm{BL}$ to TR Profile:

PosNumMed2=median(PosNum2); \% This line determines the median position number $\mathrm{j} 2=\operatorname{round}(\operatorname{PosNumMed} 2) ; \%$ The rounded midpoint of the position number

$\mathrm{f} 2=\mathrm{j} 2-20 ; \%$ The beginning of the flat middle region

$\mathrm{g} 2=\mathrm{j} 2+20 ; \%$ The end of the flat middle region

$\mathrm{e} 2=$ PosNum2(21); \% The end of the first flat region

$\mathrm{h} 2=$ PosNum2(a2-20); \% The beginning of the last flat region

$\%$ These for loops make the twenty pixels closest to the electrodes have a bias

$\%$ value of +1

for $\mathrm{i}=1: \mathrm{g} 2$;

$$
\operatorname{bias} 2(\mathrm{i})=1 \text {; }
$$

end

for $\mathrm{i}=\mathrm{h} 2: \operatorname{PosNum} 2(\mathrm{a} 2)$;

bias2(i)=1;

end

$\%$ This for loop makes the twenty pixels in the center of the profile line

$\%$ have a bias value of -1

for $\mathrm{i}=\mathrm{f} 2: \mathrm{g} 2$;

$\operatorname{bias} 2(\mathrm{i})=-1$;

end

\% These for loops make the two leftover regions have a linear bias that goes

$\%$ from +1 near the electrodes to -1 near the center

$\%$ First region of linear decay from +1 to -1

for $\mathrm{i}=\mathrm{e} 2: \mathrm{f} 2$;

bias2(i) $=((-2 /(f 2-e 2)) *(\operatorname{PosNum} 2(\mathrm{i})-\operatorname{PosNum} 2(\mathrm{e} 2)))+1$;

end 
$\%$ Second region of linear increase from -1 to +1

for $\mathrm{i}=\mathrm{g} 2: \mathrm{h} 2$;

bias $2(\mathrm{i})=((2 /(\mathrm{h} 2-\mathrm{g} 2)) *(\operatorname{PosNum} 2(\mathrm{i})-\mathrm{PosNum} 2(\mathrm{~h} 2)))+1$;

end

\%Horizontal Profile:

PosNumMed3=median(PosNum3); \% This line determines the median position number $\mathrm{j} 3=$ round(PosNumMed3); \% The rounded midpoint of the position number

e $3=$ PosNum3(70); \% This is the end of the left electrode flat region

$\mathrm{h} 3=\mathrm{PosNum} 3(\mathrm{a} 3-70) ; \%$ This is the beginning of the right electrode flat region

$\mathrm{f} 3=\mathrm{j} 3-20 ; \%$ The beginning of the flat middle region

$\mathrm{g} 3=\mathrm{j} 3+20 ; \%$ The end of the flat middle region

$\%$ These for loops make the pixels between the electrodes have a bias

$\%$ value of +1

for $\mathrm{i}=1: \mathrm{e}$;

$\operatorname{bias} 3(\mathrm{i})=1$;

end

for $\mathrm{i}=\mathrm{h} 3$ :PosNum3(a3);

$\operatorname{bias} 3(i)=1$;

end

\% This for loop makes the forty pixels in the center of the profile line

$\%$ have a bias value of -1

for $\mathrm{i}=\mathrm{f} 3: \mathrm{g} 3$;

$\operatorname{bias} 3(\mathrm{i})=-1$;

end

$\%$ These for loops make the two leftover regions have a linear bias that goes

$\%$ from +1 near the electrodes to -1 near the center

$\%$ First region of linear decay from +1 to -1

for $\mathrm{i}=\mathrm{e} 3: \mathrm{f3}$;

bias3(i) $=((-2 /($ f3-e3) $) *(\operatorname{PosNum} 3(\mathrm{i})-\mathrm{PosNum} 3(\mathrm{e} 3)))+1$;

end

$\%$ Second region of linear increase from -1 to +1

for $\mathrm{i}=\mathrm{g} 3: \mathrm{h} 3$;

bias3(i) $=((2 /(h 3-g 3)) *(\operatorname{PosNum} 3(\mathrm{i})-\mathrm{PosNum} 3(\mathrm{~h} 3)))+1$;

end

\%Vertical Profile:

PosNumMed4=median(PosNum4); \%This line determines the median position number $\mathrm{j} 4=$ round(PosNumMed4); \% The rounded midpoint of the position number e4=PosNum4(70); \% This is the end of the left electrode flat region

$\mathrm{h} 4=\mathrm{PosNum} 4(\mathrm{a} 4-70) ; \%$ This is the beginning of the right electrode flat region

$\mathrm{f} 4=\mathrm{j} 4-20 ; \%$ The beginning of the flat middle region

$\mathrm{g} 4=\mathrm{j} 4+20 ; \%$ The end of the flat middle region

$\%$ These for loops make the pixels between the electrodes have a bias

$\%$ value of +1

for $\mathrm{i}=1: \mathrm{e} 4$; 


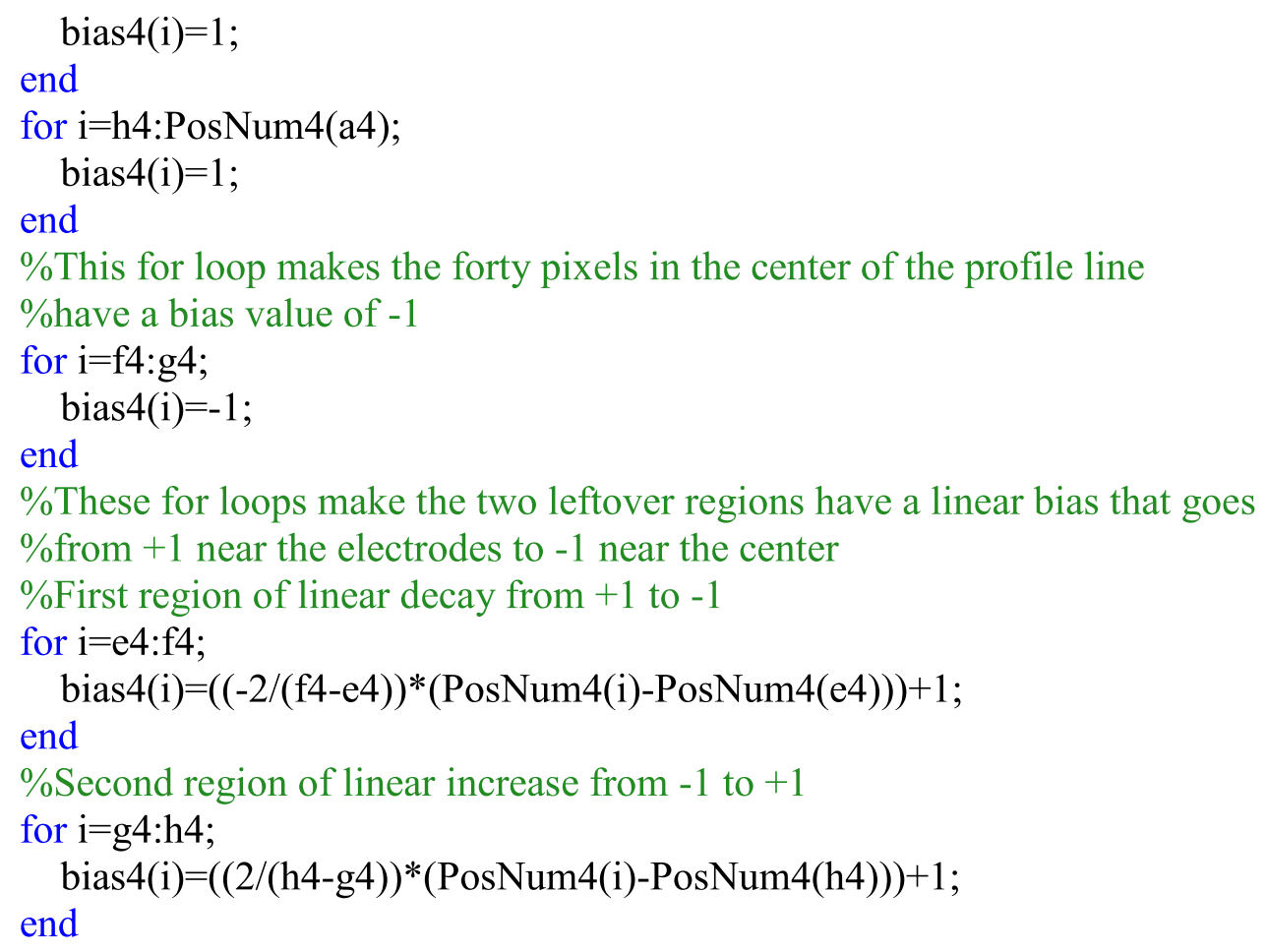




\section{biasint $2=$ sumint 2 ; \\ else}

biasint $2=$ sumint $2 . /$ avgint $2 \mathrm{~b} ; \%$ calculates the final biased intensity value by dividing the summation by the average intensity value at each frequency point

end

biasintsmooth $2=\operatorname{smooth}($ biasint 2$) ; \%$ smooths the data using a running average method

\%Horizontal Profile:

avgint $3 \mathrm{a}=$ mean(intabs3); \%calculates the average intensity value for each frequency

avgint $3 b=$ mean(avgint $3 a)$; \%calculates the average intensity value for the entire profile

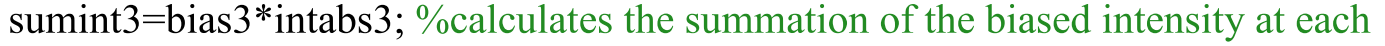

frequency point

if avgint $3 b==0$

biasint $3=$ sumint 3 ;

else

biasint $3=$ sumint $3 . /$ avgint $3 \mathrm{~b} ; \%$ calculates the final biased intensity value by dividing the summation by the average intensity value at each frequency point

end

biasintsmooth $3=$ smooth(biasint 3$) ; \%$ smooths the data using a running average method

$\%$ Vertical Profile:

avgint $4 \mathrm{a}=$ mean(intabs 4$) ; \%$ calculates the average intensity value for each frequency

avgint $4 \mathrm{~b}=$ mean(avgint $4 \mathrm{a}) ; \%$ calculates the average intensity value for the entire profile sumint $4=$ bias $4 *$ intabs $4 ; \%$ calculates the summation of the biased intensity at each

frequency point

if avgint $4 b==0$

biasint4=sumint 4 ;

else

biasint4=sumint4./avgint $4 \mathrm{~b} ; \%$ calculates the final biased intensity value by dividing the summation by the average intensity value at each frequency point

end

biasintsmooth4=smooth(biasint4); \%smooths the data using a running average method

\%This section takes the modified intensity from each profile for every

$\%$ frequency and averages them together to get a single modified intensity

$\%$ for that frequency that is a combination of all three profile lines

combinedint=zeros $(\mathrm{b} 1-3,1)$;

for $m=1: 1: b 1-3$

combinedintb $(\mathrm{m})=($ biasintsmooth $1(\mathrm{~m})+$ biasintsmooth $2(\mathrm{~m})+$ biasintsmooth $3(\mathrm{~m})+$ biasints mooth $4(\mathrm{~m})) / 4$; \%calculates the average intensity for a given timepoint

end

$\%$ To get the max intensity of the combined profile leaving the first five

$\%$ seconds as settling in time

for $m 2=1: b 1-8$ 


\section{combinedintb $2(\mathrm{~m} 2)=$ combinedintb $(\mathrm{m} 2+5)$; \\ end \\ maxcombined $=\max ($ combinedintb2 $)$; \\ mincombined $=\min ($ combinedintb 2$)$;}

$\% \%$ Plots:

$\% \% \mathrm{TL}$ to $\mathrm{BR}$ :

$\% \%$ This plots the bias versus position number for TL to BR profile

$\%$ figure

$\%$ plot(PosNum1,bias1,'r', 'LineWidth', 4);

$\%$ axis auto

$\%$ title('Bias Number versus Position Number for TL to BR Profile')

$\% \%$ This section plots the calculated biased intenisty for TL to BR profile

$\%$ figure

$\%$ plot(freq,biasintsmooth1, 'LineWidth', 4); \%Plots the velocity data versus frequency

$\%$ axis auto

$\%$ legend('Biased Intensity-Smoothed')

$\%$ title(file1)

$\%$

$\% \% \mathrm{BL}$ to $\mathrm{TR}$ :

$\% \%$ This plots the bias versus position number for BL to TR profile

$\%$ figure

$\%$ plot(PosNum2,bias2,'r', 'LineWidth', 4);

$\%$ axis auto

$\%$ title('Bias Number versus Position Number for BL to TR Profile')

$\% \%$ This section plots the calculated biased intenisty for BL to TR profile

$\%$ figure

$\%$ plot(freq,biasintsmooth2, 'LineWidth', 4); \%Plots the velocity data versus frequency

$\%$ axis auto

$\%$ legend('Biased Intensity-Smoothed',)

$\%$ title(file2)

$\%$

$\% \%$ Horizontal:

$\% \%$ This plots the bias versus position number for Horizontal profile

$\%$ figure

$\%$ plot(PosNum3,bias3,'r', 'LineWidth', 4);

$\%$ axis auto

$\%$ title('Bias Number versus Position Number for Horizontal Profile')

$\% \%$ This section plots the calculated biased intenisty for Horizontal profile

$\%$ figure

$\%$ plot(freq,biasintsmooth3, 'LineWidth', 4); \%Plots the velocity data versus frequency

$\%$ axis auto

$\%$ legend('Biased Intensity-Smoothed')

$\%$ title(file 3 )

$\%$ 
$\% \%$ Vertical:

$\% \%$ This plots the bias versus position number for Vertical profile

$\%$ figure

$\%$ plot(PosNum4,bias4,'r', 'LineWidth', 4);

$\%$ axis auto

$\%$ title('Bias Number versus Position Number for Vertical Profile')

$\% \%$ This section plots the calculated biased intenisty for Vertical profile

$\%$ figure

$\%$ plot(freq,biasintsmooth4,'LineWidth', 4); \%Plots the velocity data versus frequency

$\%$ axis auto

$\%$ legend('Biased Intensity-Smoothed')

$\%$ title(file4)

\%Combined Plots:

$\%$ This section plots all of the biased intensity profiles

$\%$ figure

$\%$ plot(freq,biasintsmooth1, 'g', freq,biasintsmooth2, 'r', freq,biasintsmooth3, 'm', freq,biasintsmooth4, 'c', 'LineWidth', 4); \%Plots the velocity data versus frequency $\%$ axis auto

$\%$ legend('Top Left to Bottom Right', 'Bottom Left to Top Right', 'Horizontal', 'Vertical')

$\%$ title('Biased Intensity Profiles')

$\%$ This section plots all of the biased intensity profiles with the averaged

$\%$ intensity

figure

plot(freq,biasintsmooth1, 'g', freq,biasintsmooth2, 'r', freq,biasintsmooth3, 'm', freq,biasintsmooth4, 'c', freq,combinedintb, 'k', 'LineWidth', 4); \%Plots the velocity data versus frequency

axis auto

legend('Top Left to Bottom Right', 'Bottom Left to Top Right', 'Horizontal', 'Vertical', 'Combined Intensity')

title('Smoothed Intensity Profiles with Combined Intensity Profile') 


\title{
9.4. Permission for Republication
}

\author{
9.4.1. Chapter 5:
}

Rightslink Printable License 7/20/12 12:10 PM

\section{JOHN WILEY AND SONS LICENSE TERMS AND CONDITIONS}

This is a License Agreement between Kaela M Leonard ("You") and John Wiley and Sons ("John Wiley and Sons") provided by Copyright Clearance Center ("CCC"). The license consists of your order details, the terms and conditions provided by John Wiley and Sons, and the payment terms and conditions. All payments must be made in full to CCC. For payment instructions, please see information listed at the bottom of this form.

License Number License date Licensed content publisher Licensed content publication Licensed content title Licensed content author Licensed content date Start page End page

Type of use Requestor type Format Portion

Will you be translating? Order reference number Total Terms and Conditions TERMS AND CONDITIONS 2953141052353

Jul 20, 2012

John Wiley and Sons

Electrophoresis

Explorations of ABO-Rh antigen expressions on erythrocyte dielectrophoresis: Changes in cross-over frequency Kaela M. Leonard,Adrienne R. Minerick Aug 23, 201125122522

Dissertation/Thesis Author of this Wiley article Print and electronic Full article No

0.00 USD

This copyrighted material is owned by or exclusively licensed to John Wiley \& Sons, Inc. or one of its group companies (each a "Wiley Company") or a society for whom a Wiley Company has exclusive publishing rights in relation to a particular journal (collectively WILEY"). By clicking "accept" in connection with completing this licensing transaction, you agree that the following terms and conditions apply to this transaction (along with the billing and payment terms and conditions established by the Copyright Clearance Center Inc., ("CCC's Billing and Payment terms and conditions"), at the time that you opened your Rightslink account (these are available at any time at http://myaccount.copyright.com)

Jul 20, 2012

https://s100.copyright.com/App/PrintableLicenseFrame.jsp?publisherID...cationID=31667\&rightID=1\&typeOfUseID=296\&targetPage=printablelicense Page 1 of 5

Rightslink Printable License 7/20/12 12:10 PM

Terms and Conditions

1. The materials you have requested permission to reproduce (the "Materials") are protected by copyright.

2. You are hereby granted a personal, non-exclusive, non-sublicensable, non-transferable, worldwide, limited

license to reproduce the Materials for the purpose specified in the licensing process. This license is for a one-time use only with a maximum distribution equal to the number that you identified in the licensing process. Any form of republication granted by this licence must be completed within two years of the date of the grant of this licence (although copies prepared before may be distributed thereafter). The Materials shall not be used in any other manner or for any other purpose. Permission is granted subject to an appropriate acknowledgement given to the author, title of the material/book/journal and the publisher. You shall also duplicate the copyright notice that appears in the Wiley publication in your use of the Material. Permission is also granted on the understanding that nowhere in the text is a previously published source acknowledged for all or part of this Material. Any third party material is expressly excluded from this permission.

3. With respect to the Materials, all rights are reserved. Except as expressly granted by the terms of the license, no part of the Materials may be copied, modified, adapted (except for minor reformatting required by the new Publication), translated, reproduced, transferred or distributed, in any form or by any means, and no derivative works may be made based on the Materials without the prior permission of the respective copyright owner. You may not alter, remove or suppress in any manner any copyright, trademark or other notices displayed by the Materials. You may not license, rent, sell, loan, lease, pledge, offer as security, transfer or assign the Materials, 
or any of the rights granted to you hereunder to any other person.

4. The Materials and all of the intellectual property rights therein shall at all times remain the exclusive property of John Wiley \& Sons Inc or one of its related companies (WILEY) or their respective licensors, and your interest therein is only that of having possession of and the right to reproduce the Materials pursuant to Section 2 herein during the continuance of this Agreement. You agree that you own no right, title or interest in or to the Materials or any of the intellectual property rights therein. You shall have no rights hereunder other than the license as provided for above in Section 2. No right, license or interest to any trademark, trade name, service mark or other branding ("Marks") of WILEY or its licensors is granted hereunder, and you agree that you shall not assert any such right, license or interest with respect thereto.

5. NEITHER WILEY NOR ITS LICENSORS MAKES ANY WARRANTY OR REPRESENTATION OF ANY KIND TO YOU OR ANY THIRD PARTY, EXPRESS, IMPLIED OR STATUTORY, WITH RESPECT TO THE MATERIALS OR THE ACCURACY OF ANY INFORMATION CONTAINED IN THE MATERIALS, INCLUDING, WITHOUT LIMITATION, ANY IMPLIED WARRANTY OF MERCHANTABILITY, ACCURACY, SATISFACTORY QUALITY, FITNESS FOR A PARTICULAR PURPOSE, USABILITY, INTEGRATION OR NON-INFRINGEMENT AND ALL SUCH WARRANTIES ARE HEREBY EXCLUDED BY WILEY AND ITS LICENSORS AND WAIVED BY YOU.

6. WILEY shall have the right to terminate this Agreement immediately upon breach of this Agreement by you. 7. You shall indemnify, defend and hold harmless WILEY, its Licensors and their respective directors, officers, agents and employees, from and against any actual or threatened claims, demands, causes of action or proceedings arising from any breach of this Agreement by you.

8. IN NO EVENT SHALL WILEY OR ITS LICENSORS BE LIABLE TO YOU OR ANY OTHER PARTY OR ANY OTHER PERSON OR ENTITY FOR ANY SPECIAL, CONSEQUENTIAL, INCIDENTAL, INDIRECT, EXEMPLARY OR PUNITIVE DAMAGES, HOWEVER CAUSED, ARISING OUT OF OR IN CONNECTION WITH THE DOWNLOADING, PROVISIONING, VIEWING OR USE OF THE MATERIALS REGARDLESS OF THE FORM OF ACTION, WHETHER FOR BREACH OF CONTRACT, BREACH OF WARRANTY, TORT, NEGLIGENCE, INFRINGEMENT OR OTHERWISE (INCLUDING, WITHOUT LIMITATION,

https://s100.copyright.com/App/PrintableLicenseFrame.jsp?publisherID...cationID=31667\&rightID=1\&typeOfUseID=296\&targetPage=printablelicense Page 2 of 5

Rightslink Printable License 7/20/12 12:10 PM

DAMAGES BASED ON LOSS OF PROFITS, DATA, FILES, USE, BUSINESS OPPORTUNITY OR

CLAIMS OF THIRD PARTIES), AND WHETHER OR NOT THE PARTY HAS BEEN ADVISED OF THE POSSIBILITY OF SUCH DAMAGES. THIS LIMITATION SHALL APPLY NOTWITHSTANDING ANY FAILURE OF ESSENTIAL PURPOSE OF ANY LIMITED REMEDY PROVIDED HEREIN.

9. Should any provision of this Agreement be held by a court of competent jurisdiction to be illegal, invalid, or unenforceable, that provision shall be deemed amended to achieve as nearly as possible the same economic effect as the original provision, and the legality, validity and enforceability of the remaining provisions of this Agreement shall not be affected or impaired thereby.

10. The failure of either party to enforce any term or condition of this Agreement shall not constitute a waiver of either party's right to enforce each and every term and condition of this Agreement. No breach under this agreement shall be deemed waived or excused by either party unless such waiver or consent is in writing signed by the party granting such waiver or consent. The waiver by or consent of a party to a breach of any provision of this Agreement shall not operate or be construed as a waiver of or consent to any other or subsequent breach by such other party.

11. This Agreement may not be assigned (including by operation of law or otherwise) by you without WILEY's prior written consent.

12. Any fee required for this permission shall be non-refundable after thirty (30) days from receipt.

13. These terms and conditions together with CCC's Billing and Payment terms and conditions (which are incorporated herein) form the entire agreement between you and WILEY concerning this licensing transaction and (in the absence of fraud) supersedes all prior agreements and representations of the parties, oral or written. This Agreement may not be amended except in writing signed by both parties. This Agreement shall be binding upon and inure to the benefit of the parties' successors, legal representatives, and authorized assigns.

14. In the event of any conflict between your obligations established by these terms and conditions and those established by CCC's Billing and Payment terms and conditions, these terms and conditions shall prevail. 15. WILEY expressly reserves all rights not specifically granted in the combination of (i) the license details provided by you and accepted in the course of this licensing transaction, (ii) these terms and conditions and (iii) CCC's Billing and Payment terms and conditions.

16. This Agreement will be void if the Type of Use, Format, Circulation, or Requestor Type was misrepresented 
during the licensing process.

17. This Agreement shall be governed by and construed in accordance with the laws of the State of New York, USA, without regards to such state's conflict of law rules. Any legal action, suit or proceeding arising out of or relating to these Terms and Conditions or the breach thereof shall be instituted in a court of competent jurisdiction in New York County in the State of New York in the United States of America and each party hereby consents and submits to the personal jurisdiction of such court, waives any objection to venue in such court and consents to service of process by registered or certified mail, return receipt requested, at the last known address of such party.

\section{Wiley Open Access Terms and Conditions}

All research articles published in Wiley Open Access journals are fully open access: immediately freely available to read, download and share. Articles are published under the terms of the Creative Commons Attribution Non Commercial License. which permits use, distribution and reproduction in any medium, provided the original work is properly cited and is not used for

https://s100.copyright.com/App/PrintableLicenseFrame.jsp?publisherID...cationID=31667\&rightID=1\&typeOfUseID=296\&targetPage=printablelicense Page 3 of 5

Rightslink Printable License 7/20/12 12:10 PM

commercial purposes. The license is subject to the Wiley Open Access terms and conditions: Wiley Open Access articles are protected by copyright and are posted to repositories and websites in accordance with the terms of the Creative Commons Attribution Non Commercial License. At the time of deposit, Wiley Open Access articles include all changes made during peer review, copyediting, and publishing. Repositories and websites that host the article are responsible for incorporating any publisher-supplied amendments or retractions issued subsequently. Wiley Open Access articles are also available without charge on Wiley's publishing platform, Wiley Online Library or any successor sites.

Use by non-commercial users

For non-commercial and non-promotional purposes individual users may access, download, copy, display and redistribute to colleagues Wiley Open Access articles, as well as adapt, translate, text- and data-mine the content subject to the following conditions:

The authors' moral rights are not compromised. These rights include the right of "paternity" (also known as "attribution" - the right for the author to be identified as such) and "integrity" (the right for the author not to have the work altered in such a way that the author's reputation or integrity may be impugned).

Where content in the article is identified as belonging to a third party, it is the obligation of the user to ensure that any reuse complies with the copyright policies of the owner of that content.

If article content is copied, downloaded or otherwise reused for non-commercial research and education purposes, a link to the appropriate bibliographic citation (authors, journal, article title, volume, issue, page numbers, DOI and the link to the definitive published version on Wiley Online Library) should be maintained.

Copyright notices and disclaimers must not be deleted.

Any translations, for which a prior translation agreement with Wiley has not been agreed, must prominently display the statement: "This is an unofficial translation of an article that appeared in a Wiley publication. The publisher has not endorsed this translation."

Use by commercial "for-profit" organisations

Use of Wiley Open Access articles for commercial, promotional, or marketing purposes requires further explicit permission from Wiley and will be subject to a fee. Commercial purposes include:

Copying or downloading of articles, or linking to such articles for further redistribution, sale or licensing;

Copying, downloading or posting by a site or service that incorporates advertising with such content; 
The inclusion or incorporation of article content in other works or services (other than normal quotations with an appropriate citation) that is then available for sale or licensing, for a fee (for example, a compilation produced for marketing purposes, inclusion in a sales pack)

Use of article content (other than normal quotations with appropriate citation) by forprofit organisations for promotional purposes

Linking to article content in e-mails redistributed for promotional, marketing or educational purposes;

https://s100.copyright.com/App/PrintableLicenseFrame.jsp?publisherID...cationID=31667\&rightID=1\&typeOfUseID=296\&targetPage=printablelicense Page 4 of 5

Rightslink Printable License 7/20/12 12:10 PM

Use for the purposes of monetary reward by means of sale, resale, licence, loan, transfer or other form of commercial exploitation such as marketing products Print reprints of Wiley Open Access articles can be purchased from:

corporatesales@wiley.com

Other Terms and Conditions:

BY CLICKING ON THE "I AGREE..." BOX, YOU ACKNOWLEDGE THAT YOU HAVE READ AND FULLY UNDERSTAND EACH OF THE SECTIONS OF AND PROVISIONS SET FORTH IN THIS AGREEMENT AND THAT YOU ARE IN AGREEMENT WITH AND ARE WILLING TO ACCEPT ALL OF YOUR OBLIGATIONS AS SET FORTH IN THIS AGREEMENT.

v1.7

If you would like to pay for this license now, please remit this license along with your payment made payable to "COPYRIGHT CLEARANCE CENTER" otherwise you will be invoiced within 48 hours of the license date. Payment should be in the form of a check or money order referencing your account number and this invoice number RLNK500822583.

Once you receive your invoice for this order, you may pay your invoice by credit card. Please follow instructions provided at that time.

Make Payment To: Copyright Clearance Center Dept 001 P.O. Box 843006 Boston, MA 02284-3006

For suggestions or comments regarding this order, contact RightsLink Customer Support: customercare@copyright.com or +1-877-622-5543 (toll free in the US) or +1- 978-646-2777.

Gratis licenses (referencing $\$ 0$ in the Total field) are free. Please retain this printable license for your reference. No payment is required.

https://s100.copyright.com/App/PrintableLicenseFrame.jsp?publisherID...cationID=31667\&rightID=1\&typeOfUseID=296\&targetPage=printablelicense Page 5 of 5

\subsubsection{Figure 2.3:}

Rightslink Printable License 8/2/12 10:15 AM

Supplier

Registered Company Number

Customer name Customer address

Elsevier Limited The Boulevard,Langford Lane Kidlington,Oxford,OX5 1GB,UK

1982084

Kaela M Leonard

1400 Townsend Drive

Houghton, MI 49931

2960810829882

Aug 02, 2012

Elsevier

Biophysical Journal

Dielectric Properties of Human Leukocyte Subpopulations Determined by Electrorotation as a Cell Separation Criterion 
Jun Yang,Ying Huang,Xujing Wang,Xiao-Bo Wang,Frederick F. Becker,Peter R.C. Gascoyne June 199976

6

833073314 reuse in a thesis/dissertation other

figures/tables/illustrations

License number License date Licensed content Licensed content Licensed content

Licensed content

Licensed content

Licensed content number

Licensed content number

Number of pages

Start Page

End Page

Type of Use

publisher publication title

author

date volume

issue

Intended publisher of new work

Portion

ELSEVIER LICENSE TERMS AND CONDITIONS

This is a License Agreement between Kaela M Leonard ("You") and Elsevier ("Elsevier") provided by Copyright Clearance Center ("CCC"). The license consists of your order details, the terms and conditions provided by Elsevier, and the payment terms and conditions.

All payments must be made in full to CCC. For payment instructions, please see information listed at the bottom of this form.

Aug 02, 2012

Number of 1

https://s100.copyright.com/App/PrintableLicenseFrame.jsp?publisherID=...icationID=22217\&rightID=1\&typeOfUseID=54\&targetPage=printablelicense Page 1 of 6

Rightslink Printable License 8/2/12 10:15 AM

Number of 1

figures/tables/illustrations Format

Are you the author of this Elsevier article?

Will you be translating? Order reference number

Title of your thesis/dissertation

Expected completion date

Estimated size (number of pages)

Elsevier VAT number Permissions price VAT/Local Sales Tax Total

Terms and Conditions

both print and electronic No

No

Alternating Current Dielectrophoretic Manipulation of Erythrocytes in Medical Microdevice Technology Aug 2012200

GB 4946272120.00 USD 0.0 USD / 0.0 GBP 0.00 USD

INTRODUCTION

1. The publisher for this copyrighted material is Elsevier. By clicking "accept" in connection with completing this licensing transaction, you agree that the following terms and conditions apply to this transaction (along with the Billing and Payment terms and conditions established by Copyright Clearance Center, Inc. ("CCC"), at the time that you opened your Rightslink account and that are available at any time at http://myaccount.copyright.com).

\section{GENERAL TERMS}


2. Elsevier hereby grants you permission to reproduce the aforementioned material subject to the terms and conditions indicated.

3. Acknowledgement: If any part of the material to be used (for example, figures) has appeared in our publication with credit or acknowledgement to another source, permission must also be sought from that source. If such permission is not obtained then that material may not be included in your publication/copies. Suitable acknowledgement to the source must be made, either as a footnote or in a reference list at the end of your publication, as follows:

"Reprinted from Publication title, Vol /edition number, Author(s), Title of article / title of chapter, Pages No., Copyright (Year), with permission from Elsevier [OR APPLICABLE SOCIETY COPYRIGHT OWNER]." Also Lancet special credit "Reprinted from The Lancet, Vol. number, Author(s), Title of article, Pages No., Copyright (Year), with permission from Elsevier."

4. Reproduction of this material is confined to the purpose and/or media for which https://s100.copyright.com/App/PrintableLicenseFrame.jsp?publisherID=...icationID=22217\&rightID=1\&typeOfUseID=54\&targetPage=printablelicense Page 2 of 6

Rightslink Printable License 8/2/12 10:15 AM

permission is hereby given.

5. Altering/Modifying Material: Not Permitted. However figures and illustrations may be altered/adapted minimally to serve your work. Any other abbreviations, additions, deletions and/or any other alterations shall be made only with prior written authorization of Elsevier Ltd. (Please contact Elsevier at permissions@elsevier.com) 6. If the permission fee for the requested use of our material is waived in this instance, please be advised that your future requests for Elsevier materials may attract a fee. 7. Reservation of Rights: Publisher reserves all rights not specifically granted in the combination of (i) the license details provided by you and accepted in the course of this licensing transaction, (ii) these terms and conditions and (iii) CCC's Billing and Payment terms and conditions.

8. License Contingent Upon Payment: While you may exercise the rights licensed immediately upon issuance of the license at the end of the licensing process for the transaction, provided that you have disclosed complete and accurate details of your proposed use, no license is finally effective unless and until full payment is received from you (either by publisher or by CCC) as provided in CCC's Billing and Payment terms and conditions. If full payment is not received on a timely basis, then any license preliminarily granted shall be deemed automatically revoked and shall be void as if never granted. Further, in the event that you breach any of these terms and conditions or any of CCC's Billing and Payment terms and conditions, the license is automatically revoked and shall be void as if never granted. Use of materials as described in a revoked license, as well as any use of the materials beyond the scope of an unrevoked license, may constitute copyright infringement and publisher reserves the right to take any and all action to protect its copyright in the materials.

9. Warranties: Publisher makes no representations or warranties with respect to the licensed material.

10. Indemnity: You hereby indemnify and agree to hold harmless publisher and CCC, and their respective officers, directors, employees and agents, from and against any 
and all claims arising out of your use of the licensed material other than as specifically authorized pursuant to this license.

11. No Transfer of License: This license is personal to you and may not be sublicensed, assigned, or transferred by you to any other person without publisher's written permission.

12. No Amendment Except in Writing: This license may not be amended except in a writing signed by both parties (or, in the case of publisher, by CCC on publisher's behalf).

13. Objection to Contrary Terms: Publisher hereby objects to any terms contained in any purchase order, acknowledgment, check endorsement or other writing prepared by you, which terms are inconsistent with these terms and conditions or CCC's Billing and Payment terms and conditions. These terms and conditions, together with CCC's Billing and Payment terms and conditions (which are incorporated herein), comprise the entire agreement

https://s100.copyright.com/App/PrintableLicenseFrame.jsp?publisherID=...icationID=22217\&rightID=1\&typeOfUseID=54\&targetPage=printablelicense Page 3 of 6

Rightslink Printable License 8/2/12 10:15 AM

between you and publisher (and CCC) concerning this licensing transaction. In the event of any conflict between your obligations established by these terms and conditions and those established by CCC's Billing and Payment terms and conditions, these terms and conditions shall control.

14. Revocation: Elsevier or Copyright Clearance Center may deny the permissions described in this License at their sole discretion, for any reason or no reason, with a full refund payable to you. Notice of such denial will be made using the contact information provided by you. Failure to receive such notice will not alter or invalidate the denial. In no event will Elsevier or Copyright Clearance Center be responsible or liable for any costs, expenses or damage incurred by you as a result of a denial of your permission request, other than a refund of the amount(s) paid by you to Elsevier and/or Copyright Clearance Center for denied permissions.

\section{LIMITED LICENSE}

The following terms and conditions apply only to specific license types:

15. Translation: This permission is granted for non-exclusive world English rights only unless your license was granted for translation rights. If you licensed translation rights you may only translate this content into the languages you requested. A professional translator must perform all translations and reproduce the content word for word preserving the integrity of the article. If this license is to re-use 1 or 2 figures then permission is granted for non-exclusive world rights in all languages.

16. Website: The following terms and conditions apply to electronic reserve and author websites: Electronic reserve: If licensed material is to be posted to website, the web site is to be password-protected and made available only to bona fide students registered on a relevant course if:

This license was made in connection with a course, This permission is granted for 1 year only. You may obtain a license for future website posting, All content posted to the web site must maintain the copyright information line on the bottom of each image, A hyper-text must be included to the Homepage of the journal from which you 
are licensing at http://www.sciencedirect.com/science/journal/xxxxx or the Elsevier homepage for books at http://www.elsevier.com, and Central Storage: This license does not include permission for a scanned version of the material to be stored in a central repository such as that provided by Heron/XanEdu.

17. Author website for journals with the following additional clauses:

All content posted to the web site must maintain the copyright information line on the bottom of each image, and the permission granted is limited to the personal version of your paper. You are not allowed to download and post the published electronic version of your article (whether PDF or HTML, proof or final version), nor may you scan the printed edition to create an electronic version. A hyper-text must be included to the Homepage of the

https://s100.copyright.com/App/PrintableLicenseFrame.jsp?publisherID=..icationID=22217\&rightID=1\&typeOfUseID=54\&targetPage=printablelicense Page 4 of 6

Rightslink Printable License 8/2/12 10:15 AM

journal from which you are licensing at

http://www.sciencedirect.com/science/journal/xxxxx . As part of our normal

production process, you will receive an e-mail notice when your article appears on

Elsevier's online service ScienceDirect (www.sciencedirect.com). That e- mail will include the article's Digital Object Identifier (DOI). This number provides the electronic link to the published article and should be included in the posting of your personal version. We ask that you wait until you receive this e-mail and have the DOI to do any posting.

Central Storage: This license does not include permission for a scanned version of the material to be stored in a central repository such as that provided by Heron/XanEdu. 18. Author website for books with the following additional clauses: Authors are permitted to place a brief summary of their work online only. A hyper-text must be included to the Elsevier homepage at http://www.elsevier.com . All content posted to the web site must maintain the copyright information line on the bottom of each image. You are not allowed to download and post the published electronic version of your chapter, nor may you scan the printed edition to create an electronic version. Central Storage: This license does not include permission for a scanned version of the material to be stored in a central repository such as that provided by Heron/XanEdu. 19. Website (regular and for author): A hyper-text must be included to the Homepage of the journal from which you are licensing at http://www.sciencedirect.com/science/journal/xxxxx. or for books to the Elsevier homepage at http://www.elsevier.com

20. Thesis/Dissertation: If your license is for use in a thesis/dissertation your thesis may be submitted to your institution in either print or electronic form. Should your thesis be published commercially, please reapply for permission. These requirements include permission for the Library and Archives of Canada to supply single copies, on demand, of the complete thesis and include permission for UMI to supply single copies, on demand, of the complete thesis. Should your thesis be published commercially, please reapply for permission.

\section{Other Conditions:}

v1.6 
If you would like to pay for this license now, please remit this license along with your payment made payable to "COPYRIGHT CLEARANCE CENTER" otherwise you will be invoiced within 48 hours of the license date. Payment should be in the form of a check or money order referencing your account number and this invoice number RLNK500830797.

Once you receive your invoice for this order, you may pay your invoice by credit card. Please follow instructions provided at that time.

Make Payment To: Copyright Clearance Center

https://s100.copyright.com/App/PrintableLicenseFrame.jsp?publisherID $=\ldots$...icationID $=22217 \&$ rightID $=1 \&$ typeOfUseID $=54 \&$ targetPage $=$ printablelicense Page 5 of 6

Rightslink Printable License 8/2/12 10:15 AM

Dept 001 P.O. Box 843006 Boston, MA 02284-3006

For suggestions or comments regarding this order, contact RightsLink Customer Support:

customercare@copyright.com or +1-877-622-5543 (toll free in the US) or +1- 978-646-2777.

Gratis licenses (referencing \$0 in the Total field) are free. Please retain this printable license for your

reference. No payment is required.

https://s100.copyright.com/App/PrintableLicenseFrame.jsp?publisherID=...icationID=22217\&rightID=1\&typeOfUseID=54\&targetPage=printablelicense Page 6 of 6

\subsubsection{Figure 2.6:}

Rightslink Printable License 7/31/12 12:24 AM

Supplier

Registered Company Number

Customer name Customer address

Elsevier Limited The Boulevard,Langford Lane Kidlington,Oxford,OX5 1GB,UK

1982084

Kaela M Leonard 1400 Townsend Drive Houghton, MI 499312959150374513 Jul 31, 2012 Elsevier Acta

Tropica Microfluidic approaches to malaria detection Peter Gascoyne,Jutamaad Satayavivad,Mathuros

Ruchirawat February 200489

3

13357369 reuse in a thesis/dissertation other

figures/tables/illustrations 1

License number

License date

Licensed content

Licensed content

Licensed content

Licensed content

Licensed content

Licensed content number

Licensed content number

Number of pages

Start Page

End Page

Type of Use

publisher publication title author date volume

issue

Intended publisher of new work

Portion

Number of figures/tables/illustrations

ELSEVIER LICENSE TERMS AND CONDITIONS

This is a License Agreement between Kaela M Leonard ("You") and Elsevier

("Elsevier") provided by Copyright Clearance Center ("CCC"). The license consists of your order details, the terms and conditions provided by Elsevier, and the payment terms and conditions.

All payments must be made in full to CCC. For payment instructions, please see information listed at the bottom of this form. 
Jul 31, 2012

https://s100.copyright.com/App/PrintableLicenseFrame.jsp?publisherID=...licationID=9917\&rightID=1\&typeOfUseID=54\&targetPage=printablelicense Page 1 of 6

Rightslink Printable License 7/31/12 12:24 AM

Format

Are you the author of this Elsevier article?

Will you be translating? Order reference number

Title of your thesis/dissertation

Expected completion date

Estimated size (number of pages)

Elsevier VAT number Permissions price VAT/Local Sales Tax Total

Terms and Conditions

both print and electronic No

No

Alternating Current Dielectrophoretic Manipulation of Erythrocytes in Medical Microdevice Technology

Aug 2012200

GB 4946272120.00 USD 0.0 USD / 0.0 GBP 0.00 USD

INTRODUCTION

1. The publisher for this copyrighted material is Elsevier. By clicking "accept" in connection with completing this licensing transaction, you agree that the following terms and conditions apply to this transaction (along with the Billing and Payment terms and conditions established by Copyright Clearance Center, Inc. ("CCC"), at the time that you opened your Rightslink account and that are available at any time at http://myaccount.copyright.com).

\section{GENERAL TERMS}

2. Elsevier hereby grants you permission to reproduce the aforementioned material subject to the terms and conditions indicated.

3. Acknowledgement: If any part of the material to be used (for example, figures) has appeared in our publication with credit or acknowledgement to another source, permission must also be sought from that source. If such permission is not obtained then that material may not be included in your publication/copies. Suitable acknowledgement to the source must be made, either as a footnote or in a reference list at the end of your publication, as follows:

"Reprinted from Publication title, Vol /edition number, Author(s), Title of article / title of chapter, Pages No., Copyright (Year), with permission from Elsevier [OR APPLICABLE SOCIETY COPYRIGHT OWNER]." Also Lancet special credit "Reprinted from The Lancet, Vol. number, Author(s), Title of article, Pages No., Copyright (Year), with permission from Elsevier."

4. Reproduction of this material is confined to the purpose and/or media for which permission is hereby given.

https://s100.copyright.com/App/PrintableLicenseFrame.jsp?publisherID=...licationID=9917\&rightID=1\&typeOfUseID=54\&targetPage=printablelicense Page 2 of 6

Rightslink Printable License 7/31/12 12:24 AM

5. Altering/Modifying Material: Not Permitted. However figures and illustrations may be altered/adapted minimally to serve your work. Any other abbreviations, additions, deletions and/or any other alterations shall be made only with prior written authorization of Elsevier Ltd. (Please contact Elsevier at permissions@elsevier.com) 6. If the permission fee for the requested use of our material is waived in this instance, please be advised that your future requests for Elsevier materials may attract a fee.

7. Reservation of Rights: Publisher reserves all rights not specifically granted in the 
combination of (i) the license details provided by you and accepted in the course of this licensing transaction, (ii) these terms and conditions and (iii) CCC's Billing and Payment terms and conditions.

8. License Contingent Upon Payment: While you may exercise the rights licensed immediately upon issuance of the license at the end of the licensing process for the transaction, provided that you have disclosed complete and accurate details of your proposed use, no license is finally effective unless and until full payment is received from you (either by publisher or by $\mathrm{CCC}$ ) as provided in CCC's Billing and Payment terms and conditions. If full payment is not received on a timely basis, then any license preliminarily granted shall be deemed automatically revoked and shall be void as if never granted. Further, in the event that you breach any of these terms and conditions or any of CCC's Billing and Payment terms and conditions, the license is automatically revoked and shall be void as if never granted. Use of materials as described in a revoked license, as well as any use of the materials beyond the scope of an unrevoked license, may constitute copyright infringement and publisher reserves the right to take any and all action to protect its copyright in the materials. 9. Warranties: Publisher makes no representations or warranties with respect to the licensed material.

10. Indemnity: You hereby indemnify and agree to hold harmless publisher and CCC, and their respective officers, directors, employees and agents, from and against any and all claims arising out of your use of the licensed material other than as specifically authorized pursuant to this license.

11. No Transfer of License: This license is personal to you and may not be sublicensed, assigned, or transferred by you to any other person without publisher's written permission.

12. No Amendment Except in Writing: This license may not be amended except in a writing signed by both parties (or, in the case of publisher, by CCC on publisher's behalf).

13. Objection to Contrary Terms: Publisher hereby objects to any terms contained in any purchase order, acknowledgment, check endorsement or other writing prepared by you, which terms are inconsistent with these terms and conditions or CCC's Billing and Payment terms and conditions. These terms and conditions, together with CCC's Billing and Payment terms and conditions (which are incorporated herein), comprise the entire agreement between you and publisher (and CCC) concerning this licensing transaction. In the event of any conflict between your obligations established by these terms and conditions and those

https://s100.copyright.com/App/PrintableLicenseFrame.jsp?publisherID=...licationID=9917\&rightID=1\&typeOfUseID=54\&targetPage=printablelicense Page 3 of 6

Rightslink Printable License 7/31/12 12:24 AM

established by CCC's Billing and Payment terms and conditions, these terms and conditions shall control.

14. Revocation: Elsevier or Copyright Clearance Center may deny the permissions described in this License at their sole discretion, for any reason or no reason, with a full refund payable to you. Notice of such denial will be made using the contact information provided by you. Failure to receive such notice will not alter or invalidate 
the denial. In no event will Elsevier or Copyright Clearance Center be responsible or liable for any costs, expenses or damage incurred by you as a result of a denial of your permission request, other than a refund of the amount(s) paid by you to Elsevier and/or Copyright Clearance Center for denied permissions.

\section{LIMITED LICENSE}

The following terms and conditions apply only to specific license types:

15. Translation: This permission is granted for non-exclusive world English rights only unless your license was granted for translation rights. If you licensed translation rights you may only translate this content into the languages you requested. A professional translator must perform all translations and reproduce the content word for word preserving the integrity of the article. If this license is to re-use 1 or 2 figures then permission is granted for non-exclusive world rights in all languages.

16. Website: The following terms and conditions apply to electronic reserve and author websites: Electronic reserve: If licensed material is to be posted to website, the web site is to be password-protected and made available only to bona fide students registered on a relevant course if:

This license was made in connection with a course, This permission is granted for 1 year only. You may obtain a license for future website posting, All content posted to the web site must maintain the copyright information line on the bottom of each image, A hyper-text must be included to the Homepage of the journal from which you are licensing at http://www.sciencedirect.com/science/journal/xxxxx or the Elsevier homepage for books at http://www.elsevier.com , and Central Storage: This license does not include permission for a scanned version of the material to be stored in a central repository such as that provided by Heron/XanEdu.

17. Author website for journals with the following additional clauses:

All content posted to the web site must maintain the copyright information line on the bottom of each image, and the permission granted is limited to the personal version of your paper. You are not allowed to download and post the published electronic version of your article (whether PDF or HTML, proof or final version), nor may you scan the printed edition to create an electronic version. A hyper-text must be included to the Homepage of the journal from which you are licensing at

http://www.sciencedirect.com/science/journal/xxxxx . As part of our normal production process, you will receive an e-mail notice when your

https://s100.copyright.com/App/PrintableLicenseFrame.jsp?publisherID=...licationID=9917\&rightID=1\&typeOfUseID=54\&targetPage=printablelicense Rightslink Printable License 7/31/12 12:24 AM

article appears on Elsevier's online service ScienceDirect (www.sciencedirect.com). That e- mail will include the article's Digital Object Identifier (DOI). This number provides the electronic link to the published article and should be included in the posting of your personal version. We ask that you wait until you receive this e-mail and have the DOI to do any posting.

Central Storage: This license does not include permission for a scanned version of the material to be stored in a central repository such as that provided by Heron/XanEdu. 18. Author website for books with the following additional clauses: Authors are permitted to place a brief summary of their work online only. A hyper-text must be 
included to the Elsevier homepage at http://www.elsevier.com . All content posted to the web site must maintain the copyright information line on the bottom of each image. You are not allowed to download and post the published electronic version of your chapter, nor may you scan the printed edition to create an electronic version. Central Storage: This license does not include permission for a scanned version of the material to be stored in a central repository such as that provided by Heron/XanEdu. 19. Website (regular and for author): A hyper-text must be included to the Homepage of the journal from which you are licensing at http:/www.sciencedirect.com/science/journal/xxxxx. or for books to the Elsevier homepage at http://www.elsevier.com

20. Thesis/Dissertation: If your license is for use in a thesis/dissertation your thesis may be submitted to your institution in either print or electronic form. Should your thesis be published commercially, please reapply for permission. These requirements include permission for the Library and Archives of Canada to supply single copies, on demand, of the complete thesis and include permission for UMI to supply single copies, on demand, of the complete thesis. Should your thesis be published commercially, please reapply for permission.

\section{Other Conditions:}

\section{v1.6}

If you would like to pay for this license now, please remit this license along with your payment made payable to "COPYRIGHT CLEARANCE CENTER" otherwise you will be invoiced within 48 hours of the license date. Payment should be in the form of a check or money order referencing your account number and this invoice number RLNK500828670.

Once you receive your invoice for this order, you may pay your invoice by credit card. Please follow instructions provided at that time.

Make Payment To: Copyright Clearance Center Dept 001 P.O. Box 843006 Boston, MA 02284-3006

https://s100.copyright.com/App/PrintableLicenseFrame.jsp?publisherID=...licationID=9917\&rightID=1\&typeOfUseID=54\&targetPage=printablelicense Page 5 of 6

Rightslink Printable License 7/31/12 12:24 AM

For suggestions or comments regarding this order, contact RightsLink Customer Support: customercare@copyright.com or +1-877-622-5543 (toll free in the US) or +1- 978-646-2777.

Gratis licenses (referencing $\$ 0$ in the Total field) are free. Please retain this printable license for your reference. No payment is required.

https://s100.copyright.com/App/PrintableLicenseFrame.jsp?publisherID= ..licationID=9917\&rightID=1\&typeOfUseID=54\&targetPage=printablelicense Page 6 of 6

\subsubsection{Figure 2.7:}

Rightslink Printable License 7/22/12 11:36 PM

Supplier

Registered Company Number

Customer name Customer address

Elsevier Limited The Boulevard,Langford Lane Kidlington,Oxford,OX5 1GB,UK

1982084

Kaela M Leonard

1400 Townsend Drive

Houghton, MI 49931

2954560269979

Jul 22, 2012

Elsevier

Biochimica et Biophysica Acta (BBA) - Biomembranes

Dielectrophoretic detection of changes in erythrocyte membranes following malarial infection

Peter Gascoyne,Ronald Pethig,Jutamaad Satayavivad,Frederick F Becker,Mathuros Ruchirawat 
31 January 19971323

2

13240252 reuse in a thesis/dissertation figures/tables/illustrations

1

License number License date Licensed content Licensed content Licensed content

Licensed content

Licensed content

Licensed content number

Licensed content number

Number of pages Start Page End Page Type of Use Portion

publisher publication title

author

date volume

issue

Number of figures/tables/illustrations

ELSEVIER LICENSE TERMS AND CONDITIONS

This is a License Agreement between Kaela M Leonard ("You") and Elsevier

("Elsevier") provided by Copyright Clearance Center ("CCC"). The license consists of your order details, the terms and conditions provided by Elsevier, and the payment terms and conditions.

All payments must be made in full to CCC. For payment instructions, please see information listed at the bottom of this form.

Jul 22, 2012

Formatboth print and electronic

https://s100.copyright.com/App/PrintableLicenseFrame.jsp?publisherID=..icationID=10431\&rightID=1\&typeOfUseID=54\&targetPage=printablelicense Page 1 of 6

Rightslink Printable License 7/22/12 11:36 PM

Formatboth print and electronic

Are you the author of this Elsevier article?

Will you be translating? Order reference number

Title of your thesis/dissertation

Expected completion date

Estimated size (number of pages)

Elsevier VAT number Permissions price VAT/Local Sales Tax Total

Terms and Conditions

No No

Alternating Current Dielectrophoretic Manipulation of Erythrocytes in Medical Microdevice Technology

Aug 2012200

GB 4946272120.00 USD 0.0 USD / 0.0 GBP 0.00 USD

INTRODUCTION

1. The publisher for this copyrighted material is Elsevier. By clicking "accept" in connection with completing this licensing transaction, you agree that the following terms and conditions apply to this transaction (along with the Billing and Payment terms and conditions established by Copyright Clearance Center, Inc. ("CCC"), at the time that you opened your Rightslink account and that are available at any time at http://myaccount.copyright.com).

GENERAL TERMS

2. Elsevier hereby grants you permission to reproduce the aforementioned material subject to the terms and conditions indicated.

3. Acknowledgement: If any part of the material to be used (for example, figures) has appeared in our publication with credit or acknowledgement to another source, permission must also be sought from that source. If such permission is not obtained then that material may not be included in your publication/copies. Suitable 
acknowledgement to the source must be made, either as a footnote or in a reference list at the end of your publication, as follows:

"Reprinted from Publication title, Vol /edition number, Author(s), Title of article / title of chapter, Pages No., Copyright (Year), with permission from Elsevier [OR APPLICABLE SOCIETY COPYRIGHT OWNER]." Also Lancet special credit "Reprinted from The Lancet, Vol. number, Author(s), Title of article, Pages No., Copyright (Year), with permission from Elsevier."

4. Reproduction of this material is confined to the purpose and/or media for which permission is hereby given.

https://s100.copyright.com/App/PrintableLicenseFrame.jsp?publisherID=...icationID=10431\&rightID=1\&typeOfUseID=54\&targetPage=printablelicense Page 2 of 6

Rightslink Printable License 7/22/12 11:36 PM

5. Altering/Modifying Material: Not Permitted. However figures and illustrations may be altered/adapted minimally to serve your work. Any other abbreviations, additions, deletions and/or any other alterations shall be made only with prior written authorization of Elsevier Ltd. (Please contact Elsevier at permissions@elsevier.com) 6. If the permission fee for the requested use of our material is waived in this instance, please be advised that your future requests for Elsevier materials may attract a fee. 7. Reservation of Rights: Publisher reserves all rights not specifically granted in the combination of (i) the license details provided by you and accepted in the course of this licensing transaction, (ii) these terms and conditions and (iii) CCC's Billing and Payment terms and conditions.

8. License Contingent Upon Payment: While you may exercise the rights licensed immediately upon issuance of the license at the end of the licensing process for the transaction, provided that you have disclosed complete and accurate details of your proposed use, no license is finally effective unless and until full payment is received from you (either by publisher or by $\mathrm{CCC}$ ) as provided in CCC's Billing and Payment terms and conditions. If full payment is not received on a timely basis, then any license preliminarily granted shall be deemed automatically revoked and shall be void as if never granted. Further, in the event that you breach any of these terms and conditions or any of CCC's Billing and Payment terms and conditions, the license is automatically revoked and shall be void as if never granted. Use of materials as described in a revoked license, as well as any use of the materials beyond the scope of an unrevoked license, may constitute copyright infringement and publisher reserves the right to take any and all action to protect its copyright in the materials.

9. Warranties: Publisher makes no representations or warranties with respect to the licensed material.

10. Indemnity: You hereby indemnify and agree to hold harmless publisher and CCC, and their respective officers, directors, employees and agents, from and against any and all claims arising out of your use of the licensed material other than as specifically authorized pursuant to this license.

11. No Transfer of License: This license is personal to you and may not be sublicensed, assigned, or transferred by you to any other person without publisher's written permission.

12. No Amendment Except in Writing: This license may not be amended except in a 
writing signed by both parties (or, in the case of publisher, by CCC on publisher's behalf).

13. Objection to Contrary Terms: Publisher hereby objects to any terms contained in any purchase order, acknowledgment, check endorsement or other writing prepared by you, which terms are inconsistent with these terms and conditions or CCC's Billing and Payment terms and conditions. These terms and conditions, together with CCC's Billing and Payment terms and conditions (which are incorporated herein), comprise the entire agreement between you and publisher (and CCC) concerning this licensing transaction. In the event of any conflict between your obligations established by these terms and conditions and those

https://s100.copyright.com/App/PrintableLicenseFrame.jsp?publisherID=...icationID=10431\&rightID=1\&typeOfUseID=54\&targetPage=printablelicense Page 3 of 6

Rightslink Printable License 7/22/12 11:36 PM

established by CCC's Billing and Payment terms and conditions, these terms and conditions shall control.

14. Revocation: Elsevier or Copyright Clearance Center may deny the permissions described in this License at their sole discretion, for any reason or no reason, with a full refund payable to you. Notice of such denial will be made using the contact information provided by you. Failure to receive such notice will not alter or invalidate the denial. In no event will Elsevier or Copyright Clearance Center be responsible or liable for any costs, expenses or damage incurred by you as a result of a denial of your permission request, other than a refund of the amount(s) paid by you to Elsevier and/or Copyright Clearance Center for denied permissions.

\section{LIMITED LICENSE}

The following terms and conditions apply only to specific license types:

15. Translation: This permission is granted for non-exclusive world English rights only unless your license was granted for translation rights. If you licensed translation rights you may only translate this content into the languages you requested. A professional translator must perform all translations and reproduce the content word for word preserving the integrity of the article. If this license is to re-use 1 or 2 figures then permission is granted for non-exclusive world rights in all languages.

16. Website: The following terms and conditions apply to electronic reserve and author websites: Electronic reserve: If licensed material is to be posted to website, the web site is to be password-protected and made available only to bona fide students registered on a relevant course if:

This license was made in connection with a course, This permission is granted for 1 year only. You may obtain a license for future website posting, All content posted to the web site must maintain the copyright information line on the bottom of each image, A hyper-text must be included to the Homepage of the journal from which you are licensing at http://www.sciencedirect.com/science/journal/xxxxx or the Elsevier homepage for books at http://www.elsevier.com, and Central Storage: This license does not include permission for a scanned version of the material to be stored in a central repository such as that provided by Heron/XanEdu.

17. Author website for journals with the following additional clauses:

All content posted to the web site must maintain the copyright information line on the 
bottom of each image, and the permission granted is limited to the personal version of your paper. You are not allowed to download and post the published electronic version of your article (whether PDF or HTML, proof or final version), nor may you scan the printed edition to create an electronic version. A hyper-text must be included to the Homepage of the journal from which you are licensing at http://www.sciencedirect.com/science/journal/xxxxx . As part of our normal production process, you will receive an e-mail notice when your https://s100.copyright.com/App/PrintableLicenseFrame.jsp?publisherID=...icationID=10431\&rightID=1\&typeOfUseID=54\&targetPage=printablelicense Page 4 of 6

Rightslink Printable License 7/22/12 11:36 PM

article appears on Elsevier's online service ScienceDirect (www.sciencedirect.com). That e- mail will include the article's Digital Object Identifier (DOI). This number provides the electronic link to the published article and should be included in the posting of your personal version. We ask that you wait until you receive this e-mail and have the DOI to do any posting.

Central Storage: This license does not include permission for a scanned version of the material to be stored in a central repository such as that provided by Heron/XanEdu. 18. Author website for books with the following additional clauses: Authors are permitted to place a brief summary of their work online only. A hyper-text must be included to the Elsevier homepage at http://www.elsevier.com . All content posted to the web site must maintain the copyright information line on the bottom of each image. You are not allowed to download and post the published electronic version of your chapter, nor may you scan the printed edition to create an electronic version. Central Storage: This license does not include permission for a scanned version of the material to be stored in a central repository such as that provided by Heron/XanEdu. 19. Website (regular and for author): A hyper-text must be included to the Homepage of the journal from which you are licensing at http://www.sciencedirect.com/science/journal/xxxxx. or for books to the Elsevier homepage at http://www.elsevier.com

20. Thesis/Dissertation: If your license is for use in a thesis/dissertation your thesis may be submitted to your institution in either print or electronic form. Should your thesis be published commercially, please reapply for permission. These requirements include permission for the Library and Archives of Canada to supply single copies, on demand, of the complete thesis and include permission for UMI to supply single copies, on demand, of the complete thesis. Should your thesis be published commercially, please reapply for permission.

\section{Other Conditions:}

v1.6

If you would like to pay for this license now, please remit this license along with your payment made payable to "COPYRIGHT CLEARANCE CENTER" otherwise you will be invoiced within 48 hours of the license date. Payment should be in the form of a check or money order referencing your account number and this invoice number RLNK500823192.

Once you receive your invoice for this order, you may pay your invoice by credit card. Please follow instructions provided at that time.

Make Payment To: Copyright Clearance Center Dept 001 P.O. Box 843006 Boston, MA 02284-3006

https://s100. copyright.com/App/PrintableLicenseFrame.jsp?publisherID=...icationID=10431\&rightID=1\&typeOfUseID=54\&targetPage=printablelicense Page 5 of 6

Rightslink Printable License 7/22/12 11:36 PM 
For suggestions or comments regarding this order, contact RightsLink Customer Support: customercare@copyright.com or +1-877-622-5543 (toll free in the US) or +1- 978-646-2777.

Gratis licenses (referencing $\mathbf{\$ 0}$ in the Total field) are free. Please retain this printable license for your reference. No payment is required.

https://s100.copyright.com/App/PrintableLicenseFrame.jsp?publisherID=...icationID=10431\&rightID=1\&typeOfUseID=54\&targetPage=printablelicense Page 6 of 6

9.4.5. Figure 3.1:

Rightslink Printable License 7/22/12 12:53 PM

JOHN WILEY AND SONS LICENSE TERMS AND CONDITIONS

This is a License Agreement between Kaela M Leonard ("You") and John Wiley and Sons ("John Wiley and Sons") provided by Copyright Clearance Center ("CCC"). The license consists of your order details, the terms and conditions provided by John Wiley and Sons, and the payment terms and conditions.

All payments must be made in full to CCC. For payment instructions, please see information listed at the bottom of this form.

License Number

License date

Licensed content publisher

Licensed content publication

Licensed content title

Licensed content author

Licensed content date

Start page

End page

Type of use

Requestor type

Format

Portion

Number of figures/tables

Number of extracts

Original Wiley figure/table number(s)

Will you be translating? Order reference number Total Terms and Conditions TERMS AND CONDITIONS

2954300980302 Jul 22, 2012 John Wiley and Sons Electrophoresis Particle separation by dielectrophoresis Peter R. C. Gascoyne,Jody Vykoukal Jul 22, 2002

19731983 Dissertation/Thesis University/Academic Print and electronic Figure/table 1

Figure 5 No

0.00 USD

This copyrighted material is owned by or exclusively licensed to John Wiley \& Sons, Inc. or one of its group companies (each a "Wiley Company") or a society for whom a Wiley Company has exclusive publishing rights in relation to a particular journal (collectively WILEY"). By clicking "accept" in connection with completing this licensing transaction, you agree that the following

Jul 22, 2012

https://s100.copyright.com/App/PrintableLicenseFrame.jsp?publisherID...cationID=31667\&rightID=1\&typeOfUseID=296\&targetPage=printablelicense Page 1 of 5

Rightslink Printable License 7/22/12 12:53 PM

terms and conditions apply to this transaction (along with the billing and payment terms and conditions established by the Copyright Clearance Center Inc., ("CCC's Billing and Payment terms and conditions"), at the time that you opened your Rightslink account (these are available at any time at http://myaccount.copyright.com) Terms and Conditions

1. The materials you have requested permission to reproduce (the "Materials") are protected by copyright.

2. You are hereby granted a personal, non-exclusive, non-sublicensable, non-transferable, worldwide, limited license to reproduce the Materials for the purpose specified in the licensing process. This license is for a one-time use only with a maximum distribution equal to the number that you identified in the licensing process. Any form of republication granted by this licence must be completed within two years of the date of the grant of this licence (although copies prepared before may be distributed thereafter). The Materials shall not be used in any 
other manner or for any other purpose. Permission is granted subject to an appropriate acknowledgement given to the author, title of the material/book/journal and the publisher. You shall also duplicate the copyright notice that appears in the Wiley publication in your use of the Material. Permission is also granted on the understanding that nowhere in the text is a previously published source acknowledged for all or part of this Material. Any third party material is expressly excluded from this permission.

3. With respect to the Materials, all rights are reserved. Except as expressly granted by the terms of the license, no part of the Materials may be copied, modified, adapted (except for minor reformatting required by the new Publication), translated, reproduced, transferred or distributed, in any form or by any means, and no derivative works may be made based on the Materials without the prior permission of the respective copyright owner. You may not alter, remove or suppress in any manner any copyright, trademark or other notices displayed by the Materials. You may not license, rent, sell, loan, lease, pledge, offer as security, transfer or assign the Materials, or any of the rights granted to you hereunder to any other person.

4. The Materials and all of the intellectual property rights therein shall at all times remain the exclusive property of John Wiley \& Sons Inc or one of its related companies (WILEY) or their respective licensors, and your interest therein is only that of having possession of and the right to reproduce the Materials pursuant to Section 2 herein during the continuance of this Agreement. You agree that you own no right, title or interest in or to the Materials or any of the intellectual property rights therein. You shall have no rights hereunder other than the license as provided for above in Section 2. No right, license or interest to any trademark, trade name, service mark or other branding ("Marks") of WILEY or its licensors is granted hereunder, and you agree that you shall not assert any such right, license or interest with respect thereto.

5. NEITHER WILEY NOR ITS LICENSORS MAKES ANY WARRANTY OR REPRESENTATION OF ANY KIND TO YOU OR ANY THIRD PARTY, EXPRESS, IMPLIED OR STATUTORY, WITH RESPECT TO THE MATERIALS OR THE ACCURACY OF ANY INFORMATION CONTAINED IN THE MATERIALS, INCLUDING, WITHOUT LIMITATION, ANY IMPLIED WARRANTY OF MERCHANTABILITY, ACCURACY, SATISFACTORY QUALITY, FITNESS FOR A PARTICULAR PURPOSE, USABILITY, INTEGRATION OR NON-INFRINGEMENT AND ALL SUCH WARRANTIES ARE HEREBY EXCLUDED BY WILEY AND ITS LICENSORS AND WAIVED BY YOU.

6. WILEY shall have the right to terminate this Agreement immediately upon breach of this Agreement by you. 7. You shall indemnify, defend and hold harmless WILEY, its Licensors and their respective directors, officers, agents and employees, from and against any actual or threatened claims, demands, causes of action or proceedings arising from any breach of this Agreement by you.

8. IN NO EVENT SHALL WILEY OR ITS LICENSORS BE LIABLE TO YOU OR ANY OTHER PARTY OR https://s100.copyright.com/App/PrintableLicenseFrame.jsp?publisherID...cationID=31667\&rightID=1\&typeOfUseID=296\&targetPage=printablelicense Page 2 of 5

Rightslink Printable License 7/22/12 12:53 PM

ANY OTHER PERSON OR ENTITY FOR ANY SPECIAL, CONSEQUENTIAL, INCIDENTAL, INDIRECT, EXEMPLARY OR PUNITIVE DAMAGES, HOWEVER CAUSED, ARISING OUT OF OR IN

CONNECTION WITH THE DOWNLOADING, PROVISIONING, VIEWING OR USE OF THE MATERIALS

REGARDLESS OF THE FORM OF ACTION, WHETHER FOR BREACH OF CONTRACT, BREACH OF

WARRANTY, TORT, NEGLIGENCE, INFRINGEMENT OR OTHERWISE (INCLUDING, WITHOUT

LIMITATION, DAMAGES BASED ON LOSS OF PROFITS, DATA, FILES, USE, BUSINESS

OPPORTUNITY OR CLAIMS OF THIRD PARTIES), AND WHETHER OR NOT THE PARTY HAS BEEN

ADVISED OF THE POSSIBILITY OF SUCH DAMAGES. THIS LIMITATION SHALL APPLY

NOTWITHSTANDING ANY FAILURE OF ESSENTIAL PURPOSE OF ANY LIMITED REMEDY

PROVIDED HEREIN.

9. Should any provision of this Agreement be held by a court of competent jurisdiction to be illegal, invalid, or unenforceable, that provision shall be deemed amended to achieve as nearly as possible the same economic effect as the original provision, and the legality, validity and enforceability of the remaining provisions of this Agreement shall not be affected or impaired thereby.

10. The failure of either party to enforce any term or condition of this Agreement shall not constitute a waiver of either party's right to enforce each and every term and condition of this Agreement. No breach under this agreement shall be deemed waived or excused by either party unless such waiver or consent is in writing signed by the party granting such waiver or consent. The waiver by or consent of a party to a breach of any provision of this Agreement shall not operate or be construed as a waiver of or consent to any other or subsequent breach by such other party.

11. This Agreement may not be assigned (including by operation of law or otherwise) by you without WILEY's prior written consent.

12. Any fee required for this permission shall be non-refundable after thirty (30) days from receipt. 
13. These terms and conditions together with CCC's Billing and Payment terms and conditions (which are incorporated herein) form the entire agreement between you and WILEY concerning this licensing transaction and (in the absence of fraud) supersedes all prior agreements and representations of the parties, oral or written. This Agreement may not be amended except in writing signed by both parties. This Agreement shall be binding upon and inure to the benefit of the parties' successors, legal representatives, and authorized assigns.

14. In the event of any conflict between your obligations established by these terms and conditions and those established by CCC's Billing and Payment terms and conditions, these terms and conditions shall prevail.

15. WILEY expressly reserves all rights not specifically granted in the combination of (i) the license details provided by you and accepted in the course of this licensing transaction, (ii) these terms and conditions and (iii) CCC's Billing and Payment terms and conditions.

16. This Agreement will be void if the Type of Use, Format, Circulation, or Requestor Type was misrepresented during the licensing process.

17. This Agreement shall be governed by and construed in accordance with the laws of the State of New York, USA, without regards to such state's conflict of law rules. Any legal action, suit or proceeding arising out of or relating to these Terms and Conditions or the breach thereof shall be instituted in a court of competent jurisdiction in New York County in the State of New York in the United States of America and each party hereby consents and submits to the personal jurisdiction of such court, waives any objection to venue in such court and consents to service of process by registered or certified mail, return receipt requested, at the last known address of such party.

Wiley Open Access Terms and Conditions

https://s100.copyright.com/App/PrintableLicenseFrame.jsp?publisherID...cationID=31667\&rightID=1\&typeOfUseID=296\&targetPage=printablelicense Page 3 of 5

Rightslink Printable License 7/22/12 12:53 PM

All research articles published in Wiley Open Access journals are fully open access: immediately freely available to read, download and share. Articles are published under the terms of the Creative Commons Attribution Non

Commercial License. which permits use, distribution and reproduction in any medium, provided the original work is properly cited and is not used for commercial purposes. The license is subject to the Wiley Open Access terms and conditions: Wiley Open Access articles are protected by copyright and are posted to repositories and websites in accordance with the terms of the Creative Commons Attribution Non Commercial License. At the time of deposit, Wiley Open Access articles include all changes made during peer review, copyediting, and publishing. Repositories and websites that host the article are responsible for incorporating any publishersupplied amendments or retractions issued subsequently.

Wiley Open Access articles are also available without charge on Wiley's publishing platform, Wiley Online

Library or any successor sites.

Use by non-commercial users

For non-commercial and non-promotional purposes individual users may access, download, copy, display and redistribute to colleagues Wiley Open Access articles, as well as adapt, translate, text- and data-mine the content subject to the following conditions:

The authors' moral rights are not compromised. These rights include the right of "paternity" (also known as "attribution" - the right for the author to be identified as such) and "integrity" (the right for the author not to have the work altered in such a way that the author's reputation or integrity may be impugned).

Where content in the article is identified as belonging to a third party, it is the obligation of the user to ensure that any reuse complies with the copyright policies of the owner of that content.

If article content is copied, downloaded or otherwise reused for non-commercial research and education purposes, a link to the appropriate bibliographic citation (authors, journal, article title, volume, issue, page numbers, DOI and the link to the definitive published version on Wiley Online Library) should be maintained. Copyright notices and disclaimers must not be deleted.

Any translations, for which a prior translation agreement with Wiley has not been agreed, must prominently display the statement: "This is an unofficial translation of an article that appeared in a Wiley publication. The publisher has not endorsed this 
translation."

\section{Use by commercial "for-profit" organisations}

Use of Wiley Open Access articles for commercial, promotional, or marketing purposes requires further explicit permission from Wiley and will be subject to a fee. Commercial purposes include:

Copying or downloading of articles, or linking to such articles for further redistribution, sale or licensing;

Copying, downloading or posting by a site or service that incorporates advertising with such content;

The inclusion or incorporation of article content in other works or services (other than normal quotations with an appropriate citation) that is then available for sale or licensing, for a fee (for example, a compilation produced for marketing purposes, inclusion in a sales pack)

https://s100.copyright.com/App/PrintableLicenseFrame.jsp?publisherID...cationID=31667\&rightID=1\&typeOfUseID=296\&targetPage=printablelicense Page 4 of 5

Rightslink Printable License 7/22/12 12:53 PM

Use of article content (other than normal quotations with appropriate citation) by forprofit organisations for promotional purposes

Linking to article content in e-mails redistributed for promotional, marketing or educational purposes;

Use for the purposes of monetary reward by means of sale, resale, licence, loan, transfer or other form of commercial exploitation such as marketing products Print reprints of Wiley Open Access articles can be purchased from:

corporatesales@wiley.com

Other Terms and Conditions:

BY CLICKING ON THE "I AGREE..." BOX, YOU ACKNOWLEDGE THAT YOU HAVE READ AND FULLY UNDERSTAND EACH OF THE SECTIONS OF AND PROVISIONS SET FORTH IN THIS AGREEMENT AND THAT YOU ARE IN AGREEMENT WITH AND ARE WILLING TO ACCEPT ALL OF YOUR OBLIGATIONS AS SET FORTH IN THIS AGREEMENT.

$\mathrm{v} 1.7$

If you would like to pay for this license now, please remit this license along with your payment made payable to "COPYRIGHT CLEARANCE CENTER" otherwise you will be invoiced within 48 hours of the license date. Payment should be in the form of a check or money order referencing your account number and this invoice number RLNK500823075.

Once you receive your invoice for this order, you may pay your invoice by credit card. Please follow instructions provided at that time.

Make Payment To: Copyright Clearance Center Dept 001 P.O. Box 843006 Boston, MA 02284-3006

For suggestions or comments regarding this order, contact RightsLink Customer Support: customercare@copyright.com or +1-877-622-5543 (toll free in the US) or +1- 978-646-2777.

Gratis licenses (referencing $\$ 0$ in the Total field) are free. Please retain this printable license for your reference. No payment is required.

https://s100.copyright.com/App/PrintableLicenseFrame.jsp?publisherID...cationID=31667\&rightID=1\&typeOfUseID=296\&targetPage=printablelicense Page 5 of 5 